\title{
Beyond poverty as a proxy: reducing inequality in infant mortality by identifying and targeting higher risk births
}

\author{
Antonio P. Ramos* \\ Chad Hazlett ${ }^{\dagger}$ \\ Stephen Smith
}

July 20, 2021

\begin{abstract}
Infant mortality remains high and uneven in much of sub-Saharan Africa. Given finite resources, reducing premature mortality requires effective tools to identifying leftbehind populations at greatest risk. While countries routinely use income- or povertybased thresholds to target policies, we examine whether models that consider other factors can substantially improve our ability to target policies to higher-risk births. Using machine learning methods, and 25 commonly available variables that can be observed prior to birth, we construct child-level risk scores for births in 22 sub-Saharan African countries. We find that targeting based on poverty, proxied by income, is only slightly better than random targeting, with the poorest 10 percent of the population experiencing approximately 10 percent of total infant mortality burden. By contrast the 10 percent of the population at highest risk according to our model accounts for 15-30\% of infants deaths, depending on country. A hypothetical intervention that can be administered to $10 \%$ of the population and prevents just $5 \%$ of the deaths that would otherwise occur, for example, would save roughly 841,000 lives if targeted to the poorest decile, but over 1.6 million if targeted using our approach.
\end{abstract}

${ }^{*}$ Research Scientist, Department of Biostatistics, Fielding School of Public Health, California Center for Population Research University of California, Los Angeles. Email: tomramos@ucla.edu( corresponding) URL: http://www.antoniopedroramos.com/

${ }^{\dagger}$ Assistant Professor, Departments of Statistics and Political Science, University of California Los Angeles. chazlett@ucla.edu URL: http://www.chadhazlett.com

${ }^{\dagger}$ PhD Candidate, Department of Statistics, University of California Los Angeles.

Email: stephensmith13424@gmail.com. 
medRxiv preprint doi: https://doi.org/10.1101/2021.07.20.21260818; this version posted July 23, 2021. The copyright holder for this preprint (which was not certified by peer review) is the author/funder, who has granted medRxiv a license to display the preprint in perpetuity.

All rights reserved. No reuse allowed without permission.

\section{Introduction}

Goal 3.2 of the Sustainable Development Goals (SDG) seeks to "end preventable deaths of newborns and children under 5 years of age, with all countries aiming to reduce neonatal mortality to at least as low as 12 per 1,000 live births and under-5 mortality to at least as low as 25 per 1,000 live births." Despite large overall improvements, including a 44\% reduction in child mortality globally from 2000 to 2015, progress has varied widely both between and within countries country to country (Rajaratnam et al., 2010; Stuckler et al., 2010; Deaton, 2020), with under-5 mortality exceeding 80 per 1,000 live births in some countries of subSaharan Africa. Even improvements in national averages are often accompanied by widening gaps among subpopulations, with more privileged groups improving their health status faster than others (Bendavid, 2014; Moser et al., 2005)

Many of these deaths are preventable with existing, low-cost technologies and interventions (Black et al., 2003; Jones et al., 2003). However, employing these solutions under budget constraints requires targeting them to those who would have otherwise died. The statistical rareness of early-life mortality thus severely limits the effectiveness of any intervention. Although multiple risk factors play a role in early-life mortality (see e.g. Mosley and Chen, 1984), many countries target policies and interventions based on one or a few risk factors, almost invariably indicators for low income or poverty. For example, cash transfer programs designed to reduce early mortality are often targeted to the poor (e.g. Glassman et al., 2013, Basset, 2008, Akresh et al., 2015). In Burkina Faso, families enrolled in conditional cash transfer schemes were required to obtain quarterly child growth monitoring at local health clinics for all children under 60 months of age (Akresh et al., 2015). The randomized controlled trial (RCT) lentils for vaccines in India targeted the poor, as have most RCTs that aim to increase vaccine uptake, good nutrition, or child health more generally (Banerjee et al., 2010). Yet, such income- or poverty-based eligibility requirements may do 
medRxiv preprint doi: https://doi.org/10.1101/2021.07.20.21260818; this version posted July 23, 2021. The copyright holder for this preprint (which was not certified by peer review) is the author/funder, who has granted medRxiv a license to display the preprint in perpetuity.

All rights reserved. No reuse allowed without permission.

little to target those actually at highest risk. For example, Ramos et al. (2020) finds in Brazil and India that $65 \%$ of all child deaths are not not among the $20 \%$ poorest, with higher values in many other countries. Such poor targeting severely limits the potential effectiveness of any life-saving intervention that can be distributed only to those considered higher risk by these approaches, failing to save as many lives as it could or to reduce inequalities in mortality risk.

In this paper we employ a variety of machine learning methods and a wide range of risk factors to more accurately estimate mortality risk in infants (under one year of age) at the individual level in 22 countries in sub-Saharan Africa. Very few studies to date have employed more than a few variables in generating risk predictions of this kind (Ramos et al., 2018, 2020; Houweling et al., 2019). We employ only observable risk factors that we deemed would reasonably be available to policymakers or health-workers in practice. We also restricted the models to employ only information that could be used for planning purposes prior to a child's birth, i.e. excluding individual health data that would need to be collected post-birth. These approaches allow us to flexibly determine which risk factors to include in different countries based on their predictive power and data availability, while also flexibly modeling the relationship between these factors and mortality. We compare our results with models that are based only on income.

\section{Methods}

\subsection{Data Sources}

We use the most recent Demographic Health Survey (DHS) for each of 22 sub-Saharan countries, provided by IPUMS Global Health. Table 1 lists these countries and their number of observations. The data used in this study come from the most recent available Demographic and Health Surveys (DHS) (https://dhsprogram.com/). These are nationally representative 
medRxiv preprint doi: https://doi.org/10.1101/2021.07.20.21260818; this version posted July 23, 2021. The copyright holder for this preprint (which was not certified by peer review) is the author/funder, who has granted medRxiv a license to display the preprint in perpetuity.

All rights reserved. No reuse allowed without permission.

surveys that have been conducted in more than 100 low and middle income countries since 1988 and they are one of the most widely source of demographic and health data available for the poorest nations.

\section{$2.2 \quad$ Data}

Our outcome is infant mortality, indicated by death before reaching one year of age. For risk factors, we chose variables on two grounds: they must be reasonably available to policymakers and health workers in-country, and relatedly, should be measurable prior to the birth of a given child, rather than depending on the health or other features of the child once borne. The latter constraint is in place so that these risk factors could be used to calculate risk in advance and deploy interventions to households, clinics, or regions with high risk. The resulting risk factors we consider are maternal age, malaria prevalence in the vicinity, head of household age and sex, parental education, cooking fuel type, floor type, toilet type, drinking water type, income, birth order, birth month, religion, bed net usage, age of first marriage, and the death of a previous child under one or five years of age. Geographic information is also used, but varies in its granularity by country. We recode variables with many categories to construct measures that are likely to perform better. For example, the DHS data initially contain 66 categories of toilet types. We simplified this to 4 categories based on the level of improvement. Appendix A provides a complete description of these coding decisions. Table 1 indicates, for each country, the set of variables (if any) that is not available in the DHS data, the number of observations (original and with complete data), the number of infant deaths, and a description of the type of geographic data available.

Finally, the DHS does not contain an indication of how an individual or household is classified relative to the poverty line in a given country, nor does it attempt to measure income. Thus rather than including such a locally-defined poverty measure, we employ the wealth-based indices provided by DHS. This measure is a factor imputed by DHS, using 
medRxiv preprint doi: https://doi.org/10.1101/2021.07.20.21260818; this version posted July 23, 2021. The copyright holder for this preprint (which was not certified by peer review) is the author/funder, who has granted medRxiv a license to display the preprint in perpetuity.

All rights reserved. No reuse allowed without permission.

Table 1: Data availability by country

\begin{tabular}{|c|c|c|c|c|c|}
\hline Country & $\mathrm{N}$ original & N complete & $\mathrm{N}$ deaths & Geography & Unavailable \\
\hline Angola & 11024 & 10829 & 472 & province (18) & age first marriage, malaria \\
\hline Benin & 10490 & 10199 & 471 & department (12) & \\
\hline Burkina Faso & 11728 & 10905 & 752 & region (13) & \\
\hline Cameroon & 9041 & 8450 & 564 & region or city (12) & \\
\hline Congo (D.R.) & 14384 & 12731 & 785 & province (11) & previous child death(s) \\
\hline Cote d'Ivoire & 5945 & 5211 & 421 & none & \\
\hline Ghana & 4548 & 4203 & 189 & none & \\
\hline Guinea & 5422 & 5223 & 396 & region $(8)$ & cooking fuel \\
\hline Kenya & 16557 & 15110 & 589 & none & pregtermin \\
\hline Lesotho & 2360 & 2127 & 145 & district (10) & bednets, malaria \\
\hline Madagascar & 9657 & 9229 & 459 & region $(22)$ & cooking fuel \\
\hline Malawi & 13633 & 13231 & 563 & region $(3)$ & malaria \\
\hline Mali & 8043 & 7913 & 475 & region $(6)$ & cooking fuel, malaria \\
\hline Mozambique & 8493 & 8026 & 537 & province (11) & \\
\hline Niger & 9650 & 9612 & 586 & region $(8)$ & religion, malaria \\
\hline Nigeria & 24222 & 23559 & 1686 & geopolitical zone (6) & \\
\hline Rwanda & 6055 & 5350 & 168 & province $(5)$ & cooking fuel \\
\hline Senegal & 9585 & 9371 & 401 & region $(14)$ & malaria \\
\hline Tanzania & 8007 & 7688 & 319 & district (10) & cooking fuel, religion, malaria \\
\hline Uganda & 12089 & 11619 & 484 & district (112) & cooking fuel, malaria \\
\hline Zambia & 10646 & 9811 & 483 & none & \\
\hline Zimbabwe & 4852 & 4630 & 236 & province (10) & malaria \\
\hline
\end{tabular}

Note: Summary of data by country. The DHS data initially include $\mathrm{N}$ original observations in each country, which falls to $\mathrm{N}$ complete when we remove missing values on the included variables. Geography indicates what type of geographic information, if any, is available and the number of units in parentheses. All other variables described in text are used in each country except where otherwise indicated under Unavailable.

principal components analysis over a number of variables including ownership of radios, televisions, and other domestic equipment; electricity and clean water; type of materials used in the walls, floor and roof; and the type of toilet in the household. Scores are calculated at the household level, separately by country.

\subsection{Modeling}

We estimate infant mortality risk for each birth in the data set. In each country, we separately run two sets of models. One is a "wealth model", described below as a proxy for what can be achieved through poverty-based targeting, and the other are flexible machine learn- 
medRxiv preprint doi: https://doi.org/10.1101/2021.07.20.21260818; this version posted July 23, 2021. The copyright holder for this preprint (which was not certified by peer review) is the author/funder, who has granted medRxiv a license to display the preprint in perpetuity. All rights reserved. No reuse allowed without permission.

ing approaches that employ 22 variables to estimate individual level mortality risk. These include random forest (rf), extreme gradient boosting over trees (xgb), kernel regularized least squares (krls), and elastic-net regularized logistic regression (elastic-logit).

Both rf (Breiman, 2001) and xgb (Chen and Guestrin, 2016) are tree-based approaches. The simplest tree-based approaches are classification and regression trees (cart, Breiman et al. 1984), which partition the input variables recursively into groups that predict the outcome level. While single-tree models like cart are easy to interpret, they often perform poorly, and small changes in the input data can have dramatic effects on the model and results. The rf and xgb approaches both improve upon such single-tree approaches by combining many trees. For rf, this is achieved by effectively averaging together the estimates of many trees, each employing only a random subset of the input variables and trained on a random subset of the observations. As applied here, each forest contains 500 trees, and the number of variables randomly sampled for each tree split (mtry) was set for each country's model using cross-validation. Under xgb, this is similarly achieved by combining many trees, but by beginning with one tree, adding the predictions of another tree trained on the errors of the prior, then repeating this process hundreds or thousands of times. We use cross-validation for each country to determine (i) the learning rate $(\eta \in(0,1)$, where smaller values help to prevent overfitting), (ii) the maximum depth of the trees (max_depth), (iii) the proportion of the variables to be considered for tree construction (colsample_bytree), (iv) the proportion of observations from the training set used for modeling (subsample), and (v) the number of iterations for the boosting procedure (nrounds). The remaining parameters were set at common default values: the minimum requirement of prediction improvement before selecting a more complex model $(\gamma)$ was set to 0 , and min_child_weight, where higher values restrict the depth of each tree based on a measure of the homogeneity of the labels within the nodes (a sign of overfitting), was set to 1 .

Kernel-based regularized least squares (krls, Hainmueller and Hazlett, 2014) is a regres- 
medRxiv preprint doi: https://doi.org/10.1101/2021.07.20.21260818; this version posted July 23, 2021. The copyright holder for this preprint (which was not certified by peer review) is the author/funder, who has granted medRxiv a license to display the preprint in perpetuity. All rights reserved. No reuse allowed without permission.

sion and classification technique that employs kernels. Such approaches effectively build a model by leveraging information about how similar observations are to each other. Each observation is treated as a potential "fence post" in the data, and the model learns how being closer to or further from each such location in the data influences the expected "height" (probability of mortality). We employ a Gaussian kernel as the measure of similarity using default values suggested in Hainmueller and Hazlett (2014). This approach also allows for a degree of interpretation, because it allows marginal effects to be determined similar to those reported in conventional regression tables.

Elastic-net logistic regression (elastic-logit, Zou and Hastie, 2005) is similar to conventional logistic regression. While ordinary logistic regression chooses coefficients $\beta_{j}$ on each variable $j$ so as to maximize the likelihood by minimizing the negative log-likelihood, elasticnet logistic regression minimizes the negative log-likelihood plus a penalty term that helps to regularize or "shrink" the model, preventing overfitting. In our case that penalty term is equal to $\lambda\left(\frac{\alpha}{2} \sum_{j}\left|\beta_{j}\right|+\frac{1-\alpha}{2} \sum_{j} \beta_{j}^{2}\right)$. The parameters $\alpha$ and $\lambda$ are chosen by cross-validation. This constitutes a compromise between what is known as ridge regression (which is penalized by the $\ell_{2}$ norm, $\sum_{j} \beta_{j}^{2}$ ), and LASSO (penalized by the $\ell_{1}$ norm, $\sum_{j}\left|\beta_{j}\right|$ ). By including the $\ell_{1}$ norm in the penalty, elastic-net has the benefit of setting some coefficients to exactly 0 , that is, selecting some variables while dropping others.

Finally, we construct a simple ensemble model in which each probability is a simple unweighted average of the probabilities produced by elastic-logit, rf, xgb, and krls. This has two primary benefits. The first is that such ensembles often have performance as good as the best sub-models and sometimes better. Second, it allows us to commit to reporting results for a single model - the ensemble model - as our premier estimate of the predictive power of these model on the test data. This is preferable to the tendency to choose the best-performing model on the test set, which can be misleading because such a choice is effectively a means of "training on the test set". 
medRxiv preprint doi: https://doi.org/10.1101/2021.07.20.21260818; this version posted July 23, 2021. The copyright holder for this preprint (which was not certified by peer review) is the author/funder, who has granted medRxiv a license to display the preprint in perpetuity.

All rights reserved. No reuse allowed without permission.

\section{Wealth model}

Our wealth-based model employs wealth information flexibly: mortality is regressed on ten indicators for decile of wealth, equivalent to finding the mean mortality level in each wealth decide. In evaluating model performance, we can consider how being in the highest risk decile, quartile, or other groups is related to mortality risk. There is no presumption that risk is necessarily decreasing or even monotonic in the level of wealth.

We note that this is a wealth model, not a poverty model based on poverty assessments made by countries, usually based on income. Neither income nor poverty-assessments themselves are available in the DHS data. This limits our ability to benchmark against a true poverty-based system. That said, our wealth model may reasonably provide an optimistic indication of what can be predicted by income or poverty-based approaches. Household income is time-sensitive, but is likely to generate risks of early mortality when it remains low on average over a long period of time, in which case wealth will also drop. Moreover, financial shocks such as large expenses or losses may have little impact on income, but substantial impact on quality of life, access to healthcare, and poverty as locally understood (see e.g. Krishna, 2004). Our wealth model is also more flexible and less coarse than poverty-based targeting systems, which typically assign individuals through a binary system (e.g. above or below the poverty line).

\section{Sample splitting and cross-validation}

Before fitting each model, we split the data into training and test sets. For each country, $80 \%$ of observations are placed in a training and validation set, while $20 \%$ are retained in a held-out testing set. For the models described above, we tune hyperparameters using either 10-fold cross-validation within the training set (for elastic-logit, rf, xgb) or leaveone-out cross-validation (for krls). The cross-validation procedure also gives a preliminary assessment of model performance. Once all modeling choices were finalized based on this 
medRxiv preprint doi: https://doi.org/10.1101/2021.07.20.21260818; this version posted July 23, 2021. The copyright holder for this preprint (which was not certified by peer review) is the author/funder, who has granted medRxiv a license to display the preprint in perpetuity.

All rights reserved. No reuse allowed without permission.

cross-validation, those models were "frozen" and we then assessed performance on the test set. This was done only once, after writing the bulk of the paper based on the cross-validation results, then updating the final values using the actual test-set results. This provides an honest assessment out-of-sample performance that prohibits "training on the test set". We report the accuracy of the models using the performance metrics described below.

\subsection{Performance metrics}

To evaluate our approach, we employ interpretable and policy-relevant metrics. A model's recall (also referred to as sensitivity) is the proportion of actual deaths that are included in the group we consider to be at risk. What we term "recall10" is defined by first determining the group of births in the top risk decile (hence the "10") according to our model, then computing the fraction of all actual infant deaths accounted for by this group. In policy terms, this corresponds to a case in which we have resources to target $10 \%$ of the population, and wish to know how much of the total mortality risk would be covered in that group. An

ineffective risk assessment that assigns risk at random would produce a recall10 of 0.10 (10\%). By contrast an effective system points us to a top risk decile that accounts for more than $10 \%$ of the mortality, perhaps $20 \%$ or $30 \%$, for example. Similarly, we report recall20, which tells us the fraction of early deaths that occurred within the top risk quintile. For both recall10 and recall20, we also report "efficiency gain", which tells us how many times more effective the ensemble model is than the benchmark wealth model.

\subsubsection{Variable importance}

Our models only seek to determine the best risk estimate possible given a set of predictors and the answers say nothing of the causal effect of those predictors on the risk of mortality. Indeed many of the risk factors, such as religion or the type of roof, are valuable not because they cause mortality but because they are expected to signal the presence of other, unobserved 
medRxiv preprint doi: https://doi.org/10.1101/2021.07.20.21260818; this version posted July 23, 2021. The copyright holder for this preprint (which was not certified by peer review) is the author/funder, who has granted medRxiv a license to display the preprint in perpetuity.

All rights reserved. No reuse allowed without permission.

factors that cause higher mortality. In other words, confounding is expected and hoped for in these models.

It is nevertheless of value to know what the models are doing to generate forecasts given the input variables. In particular we may wish to know which variables are most important in estimating the risk, for the purposes of assessing the credibility of the model or in focusing public health resources on data collection. Unfortunately, for many machine learning models it is more difficult to characterize what the model's behavior than is the case with simpler regression approaches like OLS, in which the model is fully characterized by a small number of parameters. However, some tools are readily available and provide insights into the more and less important variables. Specifically, for elastic-net we report how often the model choose a given variable and the proportion of time their coefficient is positive. For random forest, the (scaled) "variable importance" describes what fraction of the time a given variable was chosen to be included in the classification trees over which each random forest model aggregates.

\section{Results}

The primary results are recall10 and recall20 rates computed on the held-out test set (Tables 2 and $31 \cdot 1$

\subsection{Poor performance of the wealth model}

Table 2 shows the proportion of mortality that occurred among the top $10 \%$ highest predicted risk individuals. In Rwanda and Uganda, wealth is a reasonable indicator of risk, and the $10 \%$ of the population with the higher wealth-based predicted risk account for $24 \%$ and $18 \%$

\footnotetext{
${ }^{1}$ Appendix A.1 provides the same performance metrics, but using cross-validated results from the training set. Those results are extremely similar to the test set results reported here, indicating very little over-fitting in the training process.
} 
Table 2: recall10 results, test set

\begin{tabular}{cccccccc} 
Survey & wealth & elastic-logit & rf & xgb & krls & ensemble & efficiency gain \\
\hline \hline Angola 2015 & 0.11 & 0.23 & 0.23 & 0.23 & 0.22 & 0.27 & 2.50 \\
Benin 2011 & 0.13 & 0.22 & 0.31 & 0.20 & 0.21 & 0.22 & 1.75 \\
Burkina Faso 2010 & 0.12 & 0.15 & 0.21 & 0.17 & 0.15 & 0.19 & 1.61 \\
Cameroon 2011 & 0.10 & 0.18 & 0.23 & 0.17 & 0.17 & 0.24 & 2.45 \\
Congo Democratic Republic 2013-14 & 0.13 & 0.14 & 0.18 & 0.19 & 0.14 & 0.18 & 1.40 \\
Cote d'Ivoire 2011 & 0.12 & 0.21 & 0.17 & 0.27 & 0.20 & 0.24 & 2.00 \\
Ghana 2014 & 0.05 & 0.11 & 0.19 & 0.16 & 0.08 & 0.19 & 3.50 \\
Guinea 2012 & 0.11 & 0.14 & 0.20 & 0.16 & 0.15 & 0.19 & 1.67 \\
Kenya 2014 & 0.06 & 0.21 & 0.17 & 0.12 & 0.19 & 0.21 & 3.57 \\
Lesotho 2014 & 0.14 & 0.14 & 0.14 & 0.14 & 0.10 & 0.17 & 1.25 \\
Madagascar 2008 & 0.08 & 0.10 & 0.15 & 0.10 & 0.13 & 0.21 & 2.71 \\
Malawi 2016 & 0.12 & 0.12 & 0.21 & 0.20 & 0.12 & 0.19 & 1.62 \\
Mali 2012 & 0.07 & 0.20 & 0.21 & 0.23 & 0.23 & 0.23 & 3.14 \\
Mozambique 2011 & 0.09 & 0.13 & 0.21 & 0.13 & 0.13 & 0.21 & 2.20 \\
Niger 2012 & 0.12 & 0.15 & 0.13 & 0.17 & 0.13 & 0.16 & 1.36 \\
Nigeria 2013 & 0.12 & 0.21 & 0.22 & 0.19 & 0.24 & 0.25 & 2.15 \\
Rwanda 2014 & 0.24 & 0.21 & 0.09 & 0.21 & 0.21 & 0.21 & 0.88 \\
Senegal 2017 & 0.10 & 0.16 & 0.26 & 0.29 & 0.15 & 0.29 & 2.87 \\
Tanzania 2015 & 0.03 & 0.21 & 0.14 & 0.16 & 0.17 & 0.22 & 7.00
\end{tabular}

Table 3: Recall20 results, test set

\begin{tabular}{cccccccc} 
Survey & wealth & elastic-logit & rf & xgb & krls & ensemble & efficiency gain \\
\hline \hline Angola 2015 & 0.27 & 0.45 & 0.34 & 0.38 & 0.43 & 0.44 & 1.64 \\
Benin 2011 & 0.28 & 0.36 & 0.46 & 0.34 & 0.39 & 0.46 & 1.65 \\
Burkina Faso 2010 & 0.26 & 0.35 & 0.26 & 0.33 & 0.41 & 0.36 & 1.38 \\
Cameroon 2011 & 0.29 & 0.27 & 0.33 & 0.29 & 0.29 & 0.34 & 1.19 \\
Congo Democratic Republic 2013-14 & 0.20 & 0.26 & 0.32 & 0.29 & 0.29 & 0.31 & 1.55 \\
Cote d'Ivoire 2011 & 0.25 & 0.44 & 0.26 & 0.40 & 0.36 & 0.43 & 1.71 \\
Ghana 2014 & 0.16 & 0.16 & 0.22 & 0.27 & 0.16 & 0.24 & 1.50 \\
Guinea 2012 & 0.18 & 0.27 & 0.34 & 0.24 & 0.33 & 0.33 & 1.86 \\
Kenya 2014 & 0.17 & 0.26 & 0.32 & 0.25 & 0.31 & 0.32 & 1.90 \\
Lesotho 2014 & 0.24 & 0.28 & 0.28 & 0.24 & 0.14 & 0.24 & 1.00 \\
Madagascar 2008 & 0.16 & 0.26 & 0.30 & 0.24 & 0.25 & 0.31 & 1.87 \\
Malawi 2016 & 0.26 & 0.23 & 0.30 & 0.31 & 0.25 & 0.30 & 1.17 \\
Mali 2012 & 0.17 & 0.38 & 0.35 & 0.36 & 0.37 & 0.39 & 2.31 \\
Mozambique 2011 & 0.21 & 0.23 & 0.32 & 0.22 & 0.29 & 0.34 & 1.64 \\
Niger 2012 & 0.21 & 0.27 & 0.21 & 0.29 & 0.24 & 0.28 & 1.38 \\
Nigeria 2013 & 0.22 & 0.39 & 0.35 & 0.37 & 0.40 & 0.40 & 1.81 \\
Rwanda 2014 & 0.48 & 0.30 & 0.33 & 0.39 & 0.36 & 0.33 & 0.69 \\
Senegal 2017 & 0.19 & 0.30 & 0.38 & 0.39 & 0.30 & 0.40 & 2.13 \\
Tanzania 2015 & 0.11 & 0.32 & 0.29 & 0.35 & 0.25 & 0.29 & 2.57 \\
Uganda 2016 & 0.25 & 0.27 & 0.33 & 0.39 & 0.34 & 0.42 & 1.67 \\
Zambia 2013 & 0.26 & 0.32 & 0.34 & 0.31 & 0.32 & 0.31 & 1.20
\end{tabular}

of the mortality, respectively. In the other 20 countries, wealth is almost uninformative, with Recall10 values falling between just 3\% (Tanzania) and 14\% (Lesotho). Overall, the (country-wise) average recall10 of just $11 \%$ suggests that the wealth generally model does 
medRxiv preprint doi: https://doi.org/10.1101/2021.07.20.21260818; this version posted July 23, 2021. The copyright holder for this preprint (which was not certified by peer review) is the author/funder, who has granted medRxiv a license to display the preprint in perpetuity.

All rights reserved. No reuse allowed without permission.

little or no better than chance in predicting which individuals are at highest risk.

Similarly, Table 3 shows that in most countries, recall20 is only slightly above $20 \%$, averaging (country-wise) to $23 \%$. Rwanda is again an exception, with the top quintile accounting for $48 \%$ of mortality, while recall 20 rates range from $11 \%$ to $29 \%$ in the others, and are at or below chance $(20 \%)$ in 9 of the 22 countries.

\subsection{Beyond wealth: Gains from richer models}

The elastic-net logit, rf, xgb, and krls all augment the wealth model by adding the additional variables described above. These models perform similarly to each other overall, and far better than the wealth-only model. The top risk deciles according to these models account for, on average, 17-20\% of mortality (recall10); the top risk quintile accounts for $30-32 \%$ of mortality (recall20). The ensemble model performs better than any of the individual models, on average capturing $22 \%$ of the mortality in its top risk decile (recall10), and $35 \%$ in the top risk quintile (recall20). Compared to the wealth model, the ensemble model also captures 2.3 times as much of the mortality in its top risk decile (efficiency gain for recall10) and 1.6 times the mortality in its top risk quintile (efficiency gain for recall20). No single country always shows the highest recall10 across models, nor the lowest. Moreover, within given countries, the ensemble model outperforms the constituent models in many cases, does as well as the top one or two models in many others, and is never the worst performing model in that country. Thus, relying on the ensemble model not only yields better results than any other model on average, but also safeguards against cases where individual models perform poorly in a given country. 
medRxiv preprint doi: https://doi.org/10.1101/2021.07.20.21260818; this version posted July 23, 2021. The copyright holder for this preprint (which was not certified by peer review) is the author/funder, who has granted medRxiv a license to display the preprint in perpetuity.

All rights reserved. No reuse allowed without permission.

\subsection{Variable selection}

The elastic-net logistic regression model for each country automatically chooses a subset of variables to retain in the model, dropping the others. Surprisingly, every variable we provided was included in at least $36 \%$ of the country-level models. That is, at least taken across countries, there are not just a few key variables; rather the models tend to be fairly dense such that every variable is present in at least a third of the models. A few variables, though, are included especially often: most models included the previous death of a sibling in the first year $(86 \%)$ or the death of any previous sibling at any point $(76 \%) .77 \%$ of country level models included the child's gender; $73 \%$ included an urban/rural indicator. Over twothirds (68\%) of models included the mother's years of education, and many included the birth month (73\% use sine-transformed birth month; $55 \%$ use cosine-transformed birth month). Maternal age was included in $64 \%$ of models.

For random forest, the "scaled variable importance" describes what fraction of the time a given variable was chosen to be included in the classification trees over which each random forest model aggregates. Compared to elastic-net logistic regression, random forest was somewhat more selective, with over half of variables appearing in fewer than $15 \%$ of the regression trees. A few variables were particularly rarely included, such as clean cooking fuel $(3 \%)$ and minority religion (5\%). By contrast, maternal age was included nearly every time $(99.6 \%)$, followed closely by wealth percentile and it's square $(93 \%, 94 \%)$. The latter is particularly notable, as wealth percentiles were among the least commonly employed in the elastic-net logit models. Other important variables included malaria incidence rate in the area (92\%), age of household head (77\%), and mother's age at first marriage or union $(63 \%)$. 
Table 4: Variable Importance

\begin{tabular}{|c|c|c|c|}
\hline Variable & $\begin{array}{l}\text { Elastic } \\
\text { survival }\end{array}$ & $\begin{array}{c}\text { Proportion of } \\
\text { positive coefficients }\end{array}$ & $\begin{array}{l}\text { rf variable } \\
\text { importance }\end{array}$ \\
\hline previous death of sibling in first year & 0.86 & 1.00 & 0.11 \\
\hline mother's years of education & 0.77 & 0.06 & 0.39 \\
\hline sine-transformed birth month & 0.73 & 0.69 & 0.46 \\
\hline female & 0.73 & 0.00 & 0.17 \\
\hline previous death of sibling & 0.71 & 1.00 & 0.11 \\
\hline rural & 0.64 & 0.71 & 0.10 \\
\hline malaria & 0.62 & 0.75 & 0.92 \\
\hline maternal age & 0.59 & 0.08 & 1.00 \\
\hline previous pregnancy termination for mother & 0.57 & 0.75 & 0.12 \\
\hline clean water access & 0.55 & 0.58 & 0.14 \\
\hline cosine-transformed birth month & 0.55 & 0.33 & 0.48 \\
\hline log-transformed birth order & 0.55 & 0.25 & 0.54 \\
\hline mother's age at first marriage/union & 0.52 & 0.45 & 0.63 \\
\hline log-transformed number of bednets & 0.52 & 0.09 & 0.38 \\
\hline safe floor material & 0.50 & 0.18 & 0.11 \\
\hline age of household head & 0.50 & 0.64 & 0.77 \\
\hline female head of household & 0.50 & 0.55 & 0.11 \\
\hline minority religion & 0.50 & 0.70 & 0.06 \\
\hline christian & 0.35 & 0.43 & 0.08 \\
\hline muslim & 0.35 & 0.71 & 0.06 \\
\hline unimproved toilet & 0.32 & 0.86 & 0.09 \\
\hline clean cooking fuel & 0.31 & 0.20 & 0.05 \\
\hline square of wealth percentile & 0.27 & 0.00 & 0.94 \\
\hline pit toilet & 0.23 & 0.60 & 0.10 \\
\hline wealth percentile & 0.23 & 0.60 & 0.93 \\
\hline
\end{tabular}

\section{Discussion}

Across 22 countries in sub-Saharan Africa, predicting early mortality based on wealth measures alone was ineffective: those in highest $10 \%$ and $20 \%$ risk groups accounted for only $11 \%$ and $23 \%$ of mortality respectively. Fortunately, however, when wealth information is combined with other risk factors, flexible models can more accurately assess individual-level 
medRxiv preprint doi: https://doi.org/10.1101/2021.07.20.21260818; this version posted July 23, 2021. The copyright holder for this preprint (which was not certified by peer review) is the author/funder, who has granted medRxiv a license to display the preprint in perpetuity.

All rights reserved. No reuse allowed without permission.

risk of early mortality. On average across countries, the $10 \%$ of births with the highest risk as predicted by the ensemble model account for $22 \%$ of deaths, and the $20 \%$ at highest risk account for $35 \%$. Compared to the wealth-only models - which we expect are optimistic benchmarks for the poverty-based targeting approach - each country's ensemble model on average would have identified 2.3 times as many deaths in the top risk decile, and 1.6 times as many in the top $20 \%$ risk group. Senegal offers a relatively representative example, in which targeting the poorest $10 \%$ will identify only $10 \%$ of all infant deaths, while targeting the $10 \%$ at highest risk according to the ensemble model would identify $29 \%$ of all deaths.

The various models used to incorporate these risk factors - a regularized logistic regression (elastic-logit), random forest (rf), extreme gradient boosting (xgb), and kernel regularized least squares (krls) - have similar average performance. However, some models exceed others in particular countries. Using the average of predictions from these (ensemble) generates better performance on average while importantly protecting against cases where individual models performed poorly in particular countries. Particularly important to many models were variables regarding the previous death of siblings in the first year, mother's age and education, malaria prevalence, and the child's gender. The value of these variables as predictors says nothing of their causal influence, and we often expect they are predictive because they signal the presence or absence of other, hard to measure factors that influence risk.

Central to the policy-relevance of such an approach is the question of whether the variables needed to run these models are generally accessible. The risk factors we included were all chosen to be feasibly collected and employed in targeting strategies by health agencies. We avoided post-birth variables, such as birth-weight, which require real-time data. Only the sex of the child and the calendar month of birth are unknown until the birth occurs, and this would not impeded the planning or siting of interventions and materials that are expected to reduce preventable deaths.

These findings are consistent with previous studies that show that births at high risk 
medRxiv preprint doi: https://doi.org/10.1101/2021.07.20.21260818; this version posted July 23, 2021. The copyright holder for this preprint (which was not certified by peer review) is the author/funder, who has granted medRxiv a license to display the preprint in perpetuity. All rights reserved. No reuse allowed without permission.

of death exist across all socioeconomic groups, and that combining multiple risk factors improves our ability to more accurately identify the higher risk births (Ramos et al., 2018, 2020; Houweling et al., 2019). Our findings are related to a large body of literature in medicine and public health that develops risk scores to identify those at risk of some event (e.g. Mpimbaza et al., 2015; Beymer et al., 2017). To our knowledge, prior work on infant mortality using flexible models or machine learning approaches had not previously been used to develop birth-level risk scores in sub-Saharan Africa.

How beneficial might this improvement in predictive power be? We consider the potential benefit of an improved targeting system such as this for a hypothetical intervention under resource constraints. Consider an intervention that a given country can afford to administer to only $10 \%$ of births. Ideally, this would be targeted to the $10 \%$ at highest risk of early mortality. This intervention would not reduce mortality among children who would not have died anyway, but let us suppose it reduces mortality by some proportion, efficacy, among those who would have otherwise have died. In a country with $N_{\text {births }}$ per year, the number of lives saved per year would be efficacy $* \operatorname{Pr}($ death/high risk $) * N_{\text {births }} / 10$. Applying Bayes' rule, this is simply efficacy $*$ recall10 $* \operatorname{Pr}($ death $) * N_{\text {births }}$ where $\operatorname{Pr}$ (death) is the overall mortality rate expected for children born in that year (absent the intervention). Notice that the number of lives saved is proportional to the recall10 of the model used. Doubling the recall10 - which is roughly what we see for all of our models compared to the wealth model — would double the lives saved by that intervention.

Using recent estimates of the number of births and baseline mortality rates in each of these countries from World Bank Development Indicators (2021), the number of lives saved by an intervention with efficacy $=5 \%$ would be 841 thousand for the wealth model, but 1.61 million for the ensemble model. In simpler terms, the efficiency gain of roughly two for these models compared to the wealth model translates into roughly double the lives saved by an 
medRxiv preprint doi: https://doi.org/10.1101/2021.07.20.21260818; this version posted July 23, 2021. The copyright holder for this preprint (which was not certified by peer review) is the author/funder, who has granted medRxiv a license to display the preprint in perpetuity.

intervention with any particular efficacy..$^{2}$

We emphasize that all models here were trained and tuned by cross-validation on a training set, and the performance indicated here represents their performance on a test set that was completely unseen during training. If such models are to be used in practice, they would be retrained on the available data in a given country at present, but we emphasize the importance of careful tuning by cross-validation to avoid over-fitting that would lead to both over-confidence in the model's ability and, quite possibly, poor performance in reality. That the results of our cross-validated models on the training set is nearly identical to the results on the test set, however, suggests that it is possible to estimate how well a model is likely to perform on as-yet unseen data if one is careful during the cross-validation procedure.

\subsection{Limitations and Opportunities}

Our work is not without limitations and indeed it explores the limits of machine learning methods to predict infant death risk. The first limitation of note is simply that not all deaths can be predicted, particularly with readily available data. In general we find our models for the highest $10 \%$ risk group capture only $20-30 \%$ of mortality. This is beneficial and worth using for targeting since the effectiveness of an intervention is proportional to this recall rate, as shown above. However, it leaves a great deal of unpredicted mortality spread across the lower risk groups. Thus, when possible, programs must still be targeted to a much wider group in order to capture a large fraction of births that will die.

Second, the DHS sampling procedure attempts to produce a sample that is representative of the population. If these are in fact not as representative as hoped, then while the results still reflect the predictive power of these models in some population of each country, the results may not be representative of these countries as a whole.

\footnotetext{
${ }^{2}$ Note that the average efficiency gain (2.3) is not exactly the ratio of lives saved because the latter takes the relative birth rates and mortality in each country into account.
} 
medRxiv preprint doi: https://doi.org/10.1101/2021.07.20.21260818; this version posted July 23, 2021. The copyright holder for this preprint (which was not certified by peer review) is the author/funder, who has granted medRxiv a license to display the preprint in perpetuity.

All rights reserved. No reuse allowed without permission.

Third, the link between the improved predictive power of risk models and improved outcomes such as lives saved is complicated. While our simple hypothetical example above is intended only as an illustration, that exercise also reveals some of these complications. First, while the assumed the "efficacy" of a given hypothetical intervention is applied only to those individuals who would have otherwise have died, it is nevertheless possible that the even in this sense the true efficacy would not be constant across risk levels. For example, when children from wealthier families die young, it may less often be due to preventable causes, making the efficacy of any likely intervention lower. In our case this does little to jeopardize our finding: the efficacy rate need only apply to those in the top risk decile, and doing a poorer job of estimating risk would only further reduce the lives saved by reducing the effective efficacy. That is, accounting for this would only amplify the comparative benefits we describe of improved targeting. Second and more complicated, we are not incorporating targeting costs in our analysis. The calculus of the efficiency gains assumes that interventions have the same costs for each birth. In reality, costs need to be adjusted according to local conditions. For example, geographic location could be a major factor in calculating costs. Targeting children clustered by geographic location is likely much cheaper and easier than targeting children distant from each other, for example.

Finally, in the future this approach could be further improved if information of the cause of death were available. Associating each birth to a particular cause of the death could generate models that suggest targeting births with more specific, needed interventions.

\section{References}

Akresh, R., de Walque, D., and Kazianga, H. (2015). Alternative cash transfer delivery mechanism: Impacts on routine preventive health clinic visits in burkina faso. working paper series 17785, National Bureau of Economic Research. 
medRxiv preprint doi: https://doi.org/10.1101/2021.07.20.21260818; this version posted July 23, 2021. The copyright holder for this preprint (which was not certified by peer review) is the author/funder, who has granted medRxiv a license to display the preprint in perpetuity. All rights reserved. No reuse allowed without permission.

Banerjee, A., Duflo, E., Glennerster, R., and Kothari, D. (2010). Improving immunisation coverage in rural India: clustered randomised controlled evaluation of immunisation campaigns with and without incentives. British Medical Journal, 340(may17 1):c2220.

Basset, L. (2008). Can conditional cash transfer programs play a greater role in reducing child undernutrition? Technical report, World Bank.

Bendavid, E. (2014). Changes in child mortality over time across the wealth gradient in less developing counntries. Pediatrics, 134:e1551-e1559.

Beymer, M., Weiss, R. E., Sugar, C., Bourque, L. B., Gee, G. C., Morisky, D., Shu, S. B., Javanbakht, M., and Bolan, R. K. (2017). Are centers for disease control and prevention guidelines for preexposure prophylaxis specific enough? formulation of a personalized hiv risk score for pre-exposure prophylaxis initiation. Sexually Transmitted Diseases, 44(1).

Black, R., Morris, S., Bryce, J., and Venis, S. (2003). Where and why are 10 million children dying every year? commentary. The Lancet, 361:2226-34.

Breiman, L. (2001). Random forests. Machine Learning, 45:5-32.

Breiman, L., Friedman, J. H., Olshen, R. A., and Stone, C. J. (1984). Classification and Regression Trees. Wadsworth and Brooks, Monterey, CA.

Chen, T. and Guestrin, C. (2016). XGBoost: A scalable tree boosting system. In Proceedings of the 22nd ACM SIGKDD International Conference on Knowledge Discovery and Data Mining, KDD '16, pages 785-794, New York, NY, USA. ACM.

Deaton, A. (2020). The Great Escape: Health, Wealth, and the Origins of Inequality. Princeton University Press.

Glassman, A., Duran, D., Fleisher, L., Singer, D., Sturke, R., Angeles, G., Charles, J., Emrey, B., Gleason, J., Mwebsa, W., Saldana, K., Yarrow, K., and Koblinsky, M. (2013). 
medRxiv preprint doi: https://doi.org/10.1101/2021.07.20.21260818; this version posted July 23, 2021. The copyright holder for this preprint (which was not certified by peer review) is the author/funder, who has granted medRxiv a license to display the preprint in perpetuity. All rights reserved. No reuse allowed without permission.

Impact of conditional cash transfers on maternal and newborn health. Journal of Health, Population and Nutrition, 31.4 Suppl 2 (2013):S48-S66.

Hainmueller, J. and Hazlett, C. (2014). Kernel regularized least squares: Reducing misspecification bias with a flexible and interpretable machine learning approach. Political Analysis, pages 143-168.

Houweling, T. A. J., Klaveren, D. v., Das, S., Azad, K., Tripathy, P., Manandhar, D., Neuman, M., Jonge, E. d., Been, J. V., Steyerberg, E., and Costello, A. (2019). A prediction model for neonatal mortality in low- and middle-income countries: an analysis of data from population surveillance sites in india, nepal and bangladesh. International Journal of Epidemiology, 48(1):86-198.

Jones, G., Steketee, R., Black, R., Bhutta, Z., and Morris, S. (2003). How many child deaths can we prevent this year? The Lancet, 362:65-71.

Krishna, A. (2004). Escaping poverty and becoming poor: who gains, who loses, and why? World development, 32(1):121-136.

Moser, K., Leon, D., and Gwatkin, D. (2005). How does progress toward the millennium development goal affects inequality between the poorest and the least poor? an analysis of demographic and health survey. British Medical Journal, 7526:1180-1182.

Mosley, W. H. and Chen, L. C. (1984). An analytical framework for the study of child survival in developing countries. Population and Development Review, 10(7):25-45.

Mpimbaza, A., Sears, D., Sserwanga, A., Kigozi, R., Rubahika, D., Nadler, A., Yeka, A., and Dorsey, G. (2015). Admission risk score to predict inpatient pediatric mortality at four public hospitals in uganda. PLoS ONE, 10(7):e0133950. 
medRxiv preprint doi: https://doi.org/10.1101/2021.07.20.21260818; this version posted July 23, 2021. The copyright holder for this preprint (which was not certified by peer review) is the author/funder, who has granted medRxiv a license to display the preprint in perpetuity. All rights reserved. No reuse allowed without permission.

Rajaratnam, J. K., Marcus, J. R., Flaxman, A. D., Wang, H., Levin-Rector, A., Dwyer, L., Costa, M., Lopez, A. D., and Murray, C. J. L. (2010). Neonatal, postneonatal, childhood, and under-5 mortality for 187 countries, 1970-2010: a systematic analysis of progress towards millennium development goal 4. The Lancet.

Ramos, A. P., Heymann, J., and Weiss, R. (2018). Improving program targeting to combat early-life mortality by identifying high risk births in india. Population Health Metrics, $16(17)$

Ramos, A. P., Weiss, R. E., and Flores, M. (2020). Leave no child behind: Using data from 1.7 million children from 67 developing countries to measure inequality within and between groups of births and to identify left behind populations. PlosOne, (7):25-45.

Stuckler, D., Basu, S., and Mckee, M. (2010). Drivers of inequality in Millennium Development Goal progress: a statistical analysis. PLoS Medicine, 7(3):e1000241.

World Bank Development Indicators (2021). Birth rates, early mortality. Data retrieved from World Development Indicators, http://data.worldbank.org/.

Zou, H. and Hastie, T. (2005). Regularization and variable selection via the elastic net. Journal of the Royal Statistical Society, Series B, 67:301-320. 


\section{A Appendix}

\section{A.1 Cross validation results}

Table 5: Recall10 results, cross-validation on training set

\begin{tabular}{cccccccc} 
Survey & wealth & elastic-logit & rf & xgb & krls & ensemble & efficiency gain \\
\hline \hline Angola 2015 & 0.11 & 0.21 & 0.21 & 0.22 & 0.24 & 0.24 & 2.30 \\
Benin 2011 & 0.12 & 0.28 & 0.26 & 0.28 & 0.29 & 0.29 & 2.30 \\
Burkina Faso 2010 & 0.09 & 0.16 & 0.15 & 0.16 & 0.14 & 0.18 & 2.08 \\
Cameroon 2011 & 0.12 & 0.15 & 0.22 & 0.14 & 0.15 & 0.24 & 2.04 \\
Congo Democratic Republic 2013-14 & 0.09 & 0.14 & 0.19 & 0.16 & 0.17 & 0.20 & 2.27 \\
Cote d'Ivoire 2011 & 0.11 & 0.20 & 0.22 & 0.19 & 0.21 & 0.21 & 1.94 \\
Ghana 2014 & 0.06 & 0.18 & 0.13 & 0.17 & 0.16 & 0.18 & 3.11 \\
Guinea 2012 & 0.17 & 0.16 & 0.15 & 0.14 & 0.16 & 0.17 & 1.00 \\
Kenya 2014 & 0.08 & 0.15 & 0.17 & 0.13 & 0.15 & 0.16 & 2.14 \\
Lesotho 2014 & 0.07 & 0.09 & 0.16 & 0.08 & 0.15 & 0.14 & 2.00 \\
Madagascar 2008 & 0.10 & 0.24 & 0.24 & 0.24 & 0.25 & 0.27 & 2.66 \\
Malawi 2016 & 0.11 & 0.16 & 0.20 & 0.18 & 0.20 & 0.21 & 1.94 \\
Mali 2012 & 0.12 & 0.20 & 0.22 & 0.21 & 0.21 & 0.24 & 2.11 \\
Mozambique 2011 & 0.10 & 0.16 & 0.24 & 0.18 & 0.21 & 0.24 & 2.34 \\
Niger 2012 & 0.10 & 0.15 & 0.20 & 0.16 & 0.15 & 0.19 & 2.02 \\
Nigeria 2013 & 0.12 & 0.17 & 0.17 & 0.19 & 0.19 & 0.20 & 1.60 \\
Rwanda 2014 & 0.15 & 0.16 & 0.21 & 0.13 & 0.14 & 0.19 & 1.25 \\
Senegal 2017 & 0.08 & 0.15 & 0.21 & 0.21 & 0.17 & 0.22 & 2.67 \\
Tanzania 2015 & 0.09 & 0.12 & 0.18 & 0.16 & 0.14 & 0.19 & 2.18 \\
Uganda 2016 & 0.10 & 0.14 & 0.17 & 0.16 & 0.13 & 0.18 & 1.73 \\
Zambia 2013 & 0.08 & 0.15 & 0.21 & 0.19 & 0.19 & 0.21 & 2.61
\end{tabular}

Table 6: Recall20 results, cross-validation on training set

\begin{tabular}{cccccccc} 
Survey & wealth & elastic-logit & rf & xgb & krls & ensemble & efficiency gain \\
\hline \hline Angola 2015 & 0.24 & 0.39 & 0.37 & 0.40 & 0.38 & 0.42 & 1.76 \\
Benin 2011 & 0.24 & 0.45 & 0.41 & 0.45 & 0.45 & 0.47 & 1.97 \\
Burkina Faso 2010 & 0.23 & 0.31 & 0.29 & 0.31 & 0.30 & 0.30 & 1.30 \\
Cameroon 2011 & 0.25 & 0.27 & 0.33 & 0.27 & 0.27 & 0.37 & 1.45 \\
Congo Democratic Republic 2013-14 & 0.18 & 0.26 & 0.30 & 0.29 & 0.29 & 0.32 & 1.76 \\
Cote d'Ivoire 2011 & 0.20 & 0.32 & 0.35 & 0.32 & 0.33 & 0.39 & 1.99 \\
Ghana 2014 & 0.21 & 0.30 & 0.24 & 0.28 & 0.29 & 0.27 & 1.28 \\
Guinea 2012 & 0.28 & 0.27 & 0.29 & 0.30 & 0.27 & 0.32 & 1.12 \\
Kenya 2014 & 0.17 & 0.27 & 0.28 & 0.27 & 0.25 & 0.30 & 1.80 \\
Lesotho 2014 & 0.14 & 0.22 & 0.32 & 0.18 & 0.25 & 0.27 & 1.94 \\
Madagascar 2008 & 0.22 & 0.38 & 0.36 & 0.39 & 0.39 & 0.42 & 1.90 \\
Malawi 2016 & 0.22 & 0.30 & 0.31 & 0.32 & 0.32 & 0.35 & 1.63 \\
Mali 2012 & 0.22 & 0.36 & 0.35 & 0.36 & 0.38 & 0.37 & 1.72 \\
Mozambique 2011 & 0.24 & 0.29 & 0.37 & 0.33 & 0.32 & 0.37 & 1.53 \\
Niger 2012 & 0.20 & 0.31 & 0.31 & 0.29 & 0.28 & 0.33 & 1.71 \\
Nigeria 2013 & 0.25 & 0.34 & 0.29 & 0.34 & 0.33 & 0.34 & 1.35 \\
Rwanda 2014 & 0.26 & 0.36 & 0.30 & 0.27 & 0.31 & 0.32 & 1.23 \\
Senegal 2017 & 0.20 & 0.30 & 0.31 & 0.33 & 0.31 & 0.34 & 1.68 \\
Tanzania 2015 & 0.16 & 0.23 & 0.31 & 0.29 & 0.25 & 0.26 & 1.63 \\
Uganda 2016 & 0.22 & 0.24 & 0.28 & 0.29 & 0.24 & 0.31 & 1.37
\end{tabular}


medRxiv preprint doi: https://doi.org/10.1101/2021.07.20.21260818; this version posted July 23, 2021. The copyright holder for this preprint (which was not certified by peer review) is the author/funder, who has granted medRxiv a license to display the preprint in perpetuity.

All rights reserved. No reuse allowed without permission. 


\section{A.2 Variable construction}

Table 7: Variable information

\begin{tabular}{|c|c|c|c|}
\hline Variable & Type & Range of Values & Description \\
\hline kidbordlog & Numeric & {$[0.6931,2.9444]$} & $\begin{array}{l}\text { Natural logarithm of the } \\
\text { child's birth order }\end{array}$ \\
\hline pregtermin & Categorical & $\{$ NoPregTerm, PregTerm $\}$ & $\begin{array}{l}\text { Ever had pregnancy } \\
\text { terminate }\end{array}$ \\
\hline agefrstmar & Numeric & {$[1,49]$} & $\begin{array}{l}\text { Women's age at start of } \\
\text { first marriage or union }\end{array}$ \\
\hline hheadagehh & Numeric & {$[8,95]$} & Age of household head \\
\hline urban & Categorical & $\{$ Urban, Rural $\}$ & Urban or rural living status \\
\hline kidsex & Categorical & $\{$ girl, boy $\}$ & Sex of child \\
\hline hheadsex & Categorical & $\{$ female, male $\}$ & Sex of household head \\
\hline edyrtotal & Numeric & {$[1,25]$} & Total years educated \\
\hline maternal_age_month & Numeric & {$[135,584]$} & $\begin{array}{l}\text { Age of mother calculated in } \\
\text { months }\end{array}$ \\
\hline drinkwtr_new & Categorical & $\{$ bad, good $\}$ & $\begin{array}{l}\text { Main source of drinking } \\
\text { water }\end{array}$ \\
\hline floor_new & Categorical & $\{$ unsafe, safe $\}$ & Main material of floor \\
\hline cookfuel_new & Categorical & $\{$ unclean, clean $\}$ & $\begin{array}{l}\text { Type of fuel used for } \\
\text { cooking }\end{array}$ \\
\hline toilettype_new & Categorical & $\{$ flush, pit, unimproved $\}$ & Type of toilet \\
\hline religion_new & Categorical & $\begin{array}{l}\text { \{Buddhist, Christian, } \\
\text { Hindu, Other }\}\end{array}$ & Religion of mother \\
\hline wealths & Numeric & {$[-16.3862,32.4577]$} & $\begin{array}{l}\text { Wealth index categorical } \\
\text { score }\end{array}$ \\
\hline kidbirthmo & Categorical & $\{1,2, \hat{a} \breve{S}, 12\}$ & Month of the child's birth \\
\hline kidbirthmo_sin & Numeric & {$[-1,1]$} & $\begin{array}{l}\text { Sine transformation of } \\
\text { child's birth_month }\end{array}$ \\
\hline kidbirthmo_cos & Numeric & {$[-1,1]$} & $\begin{array}{l}\text { Cosine transformation of } \\
\text { child's birth month }\end{array}$ \\
\hline bednetnum & Numeric & $\{0,1,2,3,4,5,6,7+\}$ & $\begin{array}{l}\text { Number of mosquito nets } \\
\text { owned by the household }\end{array}$ \\
\hline bednetnum_log & Numeric & {$[0.69,2.2]$} & $\begin{array}{l}\text { Natural logarithm of } \\
\text { bednetnum }+1\end{array}$ \\
\hline prev_death & Categorical & $\{$ no, yes $\}$ & $\begin{array}{l}\text { Mother experienced } \\
\text { previous death of child } \\
¡ 12 \mathrm{mo}\end{array}$ \\
\hline prev_death_full & Categorical & $\{$ no, yes $\}$ & $\begin{array}{l}\text { Mother experienced } \\
\text { previous death of child ¡5yr }\end{array}$ \\
\hline district & Categorical & Depends on the country & $\begin{array}{l}\text { District where the } \\
\text { household lived at the time } \\
\text { of survey }\end{array}$ \\
\hline Province/State & Categorical & Depends on the country & $\begin{array}{l}\text { State or Province where the } \\
\text { household lived at the time } \\
\text { of survey }\end{array}$ \\
\hline wealthp2 & Categorical & $\{1,2,3,4,5\}$ & $\begin{array}{l}\text { Wealth quintile based on } \\
\text { wealths (by country) }\end{array}$ \\
\hline
\end{tabular}


medRxiv preprint doi: https://doi.org/10.1101/2021.07.20.21260818; this version posted July 23, 2021. The copyright holder for this preprint (which was not certified by peer review) is the author/funder, who has granted medRxiv a license to display the preprint in perpetuity.

\section{B Online Supplement}

\section{B.1 Country-by-Country Results}

Efficiency gain is measured by the ratio of Catch 10 Results for each algorithm compared to the Catch 10 Results for using wealth alone.

Table 8: Detailed Results by Country

\section{Angola}

$\begin{array}{cccccccc}\text { Elastic Net } & 0.212 & 0.394 & 0.664 & 0.012 & 1.276 & 0.933 & 2.001 \\ \text { Ensemble } & 0.244 & 0.424 & 0.683 & 0.014 & 1.399 & 0.934 & 2.300 \\ \text { KRLS } & 0.236 & 0.384 & 0.669 & 0.016 & 1.379 & 0.934 & 2.227 \\ \text { Random Forest } & 0.214 & 0.368 & 0.641 & 0.013 & 1.904 & 0.933 & 2.024 \\ \text { Wealth } & 0.106 & 0.241 & 0.560 & 0.207 & 15.020 & 0.928 & 1.000 \\ \text { XGB } & 0.225 & 0.397 & 0.666 & 0.016 & 1.379 & 0.934 & 2.124\end{array}$

Benin

$\begin{array}{cccccccc}\text { Elastic Net } & 0.284 & 0.454 & 0.678 & 0.026 & 1.568 & 0.936 & 2.276 \\ \text { Ensemble } & 0.287 & 0.475 & 0.685 & 0.023 & 1.590 & 0.935 & 2.299 \\ \text { KRLS } & 0.292 & 0.449 & 0.674 & 0.021 & 1.475 & 0.936 & 2.338 \\ \text { Random Forest } & 0.260 & 0.411 & 0.662 & 0.022 & 2.046 & 0.935 & 2.083 \\ \text { Wealth } & 0.125 & 0.242 & 0.537 & 0.118 & 2.244 & 0.928 & 1.000 \\ \text { XGB } & 0.278 & 0.446 & 0.666 & 0.024 & 1.518 & 0.935 & 2.233\end{array}$

Burkina Faso

Elastic Net

0.161

0.307

$0.608 \quad 0.008$

1.120

0.920

1.866

Ensemble

0.179

0.304

$0.626 \quad 0.010$

$1.175 \quad 0.921$

2.076 
medRxiv preprint doi: https://doi.org/10.1101/2021.07.20.21260818; this version posted July 23, 2021. The copyright holder for this preprint (which was not certified by peer review) is the author/funder, who has granted medRxiv a license to display the preprint in perpetuity.

All rights reserved. No reuse allowed without permission.

Table 8: Detailed Results by Country (continued)

\begin{tabular}{cccccccc}
\hline Algorithm & Recall 10 & Recall 20 & ROC & MRD & MRR & F1 & Efficiency Gain \\
\hline KRLS & 0.145 & 0.297 & 0.611 & 0.010 & 1.152 & 0.919 & 1.673 \\
Random Forest & 0.155 & 0.287 & 0.581 & 0.009 & 1.349 & 0.920 & 1.789 \\
Wealth & 0.086 & 0.234 & 0.553 & 0.147 & 1.958 & 0.914 & 1.000 \\
XGB & 0.159 & 0.306 & 0.620 & 0.013 & 1.188 & 0.920 & 1.845
\end{tabular}

Cameroon

$\begin{array}{cccccccc}\text { Elastic Net } & 0.153 & 0.272 & 0.584 & 0.006 & 1.085 & 0.921 & 1.304 \\ \text { Ensemble } & 0.239 & 0.365 & 0.620 & 0.021 & 1.313 & 0.926 & 2.040 \\ \text { KRLS } & 0.146 & 0.266 & 0.584 & 0.005 & 1.074 & 0.920 & 1.247 \\ \text { Random Forest } & 0.217 & 0.327 & 0.598 & 0.062 & 1.827 & 0.925 & 1.850 \\ \text { Wealth } & 0.117 & 0.252 & 0.547 & 0.137 & 2.072 & 0.918 & 1.000 \\ \text { XGB } & 0.142 & 0.274 & 0.595 & 0.013 & 1.199 & 0.920 & 1.210\end{array}$

Congo Democratic Republic

$\begin{array}{cccccccc}\text { Elastic Net } & 0.145 & 0.263 & 0.557 & 0.004 & 1.064 & 0.922 & 1.625 \\ \text { Ensemble } & 0.202 & 0.325 & 0.604 & 0.008 & 1.162 & 0.926 & 2.266 \\ \text { KRLS } & 0.174 & 0.293 & 0.588 & 0.008 & 1.126 & 0.924 & 1.945 \\ \text { Random Forest } & 0.190 & 0.296 & 0.581 & 0.015 & 1.618 & 0.925 & 2.124 \\ \text { Wealth } & 0.089 & 0.185 & 0.520 & 0.064 & 1.503 & 0.919 & 1.000 \\ \text { XGB } & 0.162 & 0.294 & 0.590 & 0.007 & 1.116 & 0.923 & 1.821\end{array}$

\section{Cote d'Ivoire}

$\begin{array}{cccccccr}\text { Elastic Net } & 0.205 & 0.321 & 0.629 & 0.016 & 1.205 & 0.920 & 1.918 \\ \text { Ensemble } & 0.208 & 0.395 & 0.654 & 0.018 & 1.241 & 0.919 & 1.943 \\ \text { KRLS } & 0.208 & 0.330 & 0.638 & 0.016 & 1.205 & 0.920 & 1.947\end{array}$


medRxiv preprint doi: https://doi.org/10.1101/2021.07.20.21260818; this version posted July 23, 2021. The copyright holder for this preprint (which was not certified by peer review) is the author/funder, who has granted medRxiv a license to display the preprint in perpetuity.

All rights reserved. No reuse allowed without permission.

Table 8: Detailed Results by Country (continued)

\begin{tabular}{cccccccc}
\hline Algorithm & Recall 10 & Recall 20 & ROC & MRD & MRR & F1 & Efficiency Gain \\
\hline Random Forest & 0.217 & 0.353 & 0.622 & 0.024 & 1.432 & 0.921 & 2.027 \\
Wealth & 0.107 & 0.199 & 0.529 & 0.076 & 2.132 & 0.911 & 1.000 \\
XGB & 0.193 & 0.324 & 0.619 & 0.015 & 1.185 & 0.919 & 1.806
\end{tabular}

\section{Ghana}

$\begin{array}{cccccccc}\text { Elastic Net } & 0.185 & 0.304 & 0.519 & 0.002 & 1.048 & 0.932 & 3.127 \\ \text { Ensemble } & 0.185 & 0.271 & 0.539 & 0.007 & 1.161 & 0.931 & 3.127 \\ \text { KRLS } & 0.165 & 0.290 & 0.567 & 0.000 & 1.009 & 0.931 & 2.796 \\ \text { Random Forest } & 0.132 & 0.243 & 0.556 & 0.019 & 1.530 & 0.929 & 2.232 \\ \text { Wealth } & 0.059 & 0.210 & 0.568 & -0.038 & 0.889 & 0.926 & 1.000 \\ \text { XGB } & 0.172 & 0.285 & 0.539 & 0.006 & 1.135 & 0.931 & 2.901\end{array}$

\section{Guinea}

$\begin{array}{cccccccc}\text { Elastic Net } & 0.157 & 0.274 & 0.576 & 0.005 & 1.070 & 0.918 & 0.926 \\ \text { Ensemble } & 0.170 & 0.318 & 0.606 & 0.009 & 1.134 & 0.918 & 1.001 \\ \text { KRLS } & 0.157 & 0.274 & 0.582 & 0.005 & 1.071 & 0.918 & 0.925 \\ \text { Random Forest } & 0.145 & 0.287 & 0.582 & 0.012 & 1.301 & 0.917 & 0.853 \\ \text { Wealth } & 0.170 & 0.283 & 0.574 & 0.242 & 2.981 & 0.919 & 1.000 \\ \text { XGB } & 0.139 & 0.296 & 0.599 & 0.013 & 1.171 & 0.916 & 0.819\end{array}$

\section{Kenya}

$\begin{array}{cccccccc}\text { Elastic Net } & 0.148 & 0.271 & 0.524 & 0.001 & 1.025 & 0.932 & 1.948 \\ \text { Ensemble } & 0.163 & 0.301 & 0.573 & 0.003 & 1.088 & 0.932 & 2.143 \\ \text { KRLS } & 0.150 & 0.250 & 0.536 & 0.000 & 1.005 & 0.932 & 1.975 \\ \text { Random Forest } & 0.168 & 0.282 & 0.564 & 0.009 & 1.408 & 0.933 & 2.199\end{array}$


medRxiv preprint doi: https://doi.org/10.1101/2021.07.20.21260818; this version posted July 23, 2021. The copyright holder for this preprint (which was not certified by peer review) is the author/funder, who has granted medRxiv a license to display the preprint in perpetuity.

All rights reserved. No reuse allowed without permission.

Table 8: Detailed Results by Country (continued)

\begin{tabular}{cccccccc}
\hline Algorithm & Recall 10 & Recall 20 & ROC & MRD & MRR & F1 & Efficiency Gain \\
\hline Wealth & 0.076 & 0.167 & 0.542 & -0.028 & 0.882 & 0.929 & 1.000 \\
XGB & 0.127 & 0.273 & 0.558 & 0.002 & 1.046 & 0.931 & 1.668 \\
Lesotho & & & & & & & \\
Elastic Net & 0.094 & 0.214 & 0.575 & 0.001 & 1.011 & 0.916 & 1.378 \\
Ensemble & 0.138 & 0.267 & 0.586 & 0.005 & 1.076 & 0.918 & 2.022 \\
KRLS & 0.145 & 0.249 & 0.548 & 0.001 & 1.017 & 0.920 & 2.133 \\
Random Forest & 0.162 & 0.317 & 0.582 & 0.013 & 1.344 & 0.921 & 2.378 \\
Wealth & 0.068 & 0.137 & 0.583 & -0.026 & 0.859 & 0.914 & 1.000 \\
XGB & 0.077 & 0.181 & 0.522 & 0.003 & 1.047 & 0.915 & 1.133
\end{tabular}

Madagascar

$\begin{array}{cccccccc}\text { Elastic Net } & 0.242 & 0.378 & 0.629 & 0.013 & 1.270 & 0.933 & 2.339 \\ \text { Ensemble } & 0.274 & 0.424 & 0.656 & 0.024 & 1.473 & 0.934 & 2.652 \\ \text { KRLS } & 0.250 & 0.391 & 0.642 & 0.014 & 1.288 & 0.933 & 2.417 \\ \text { Random Forest } & 0.236 & 0.361 & 0.635 & 0.052 & 1.933 & 0.932 & 2.285 \\ \text { Wealth } & 0.103 & 0.223 & 0.549 & 0.126 & 1.532 & 0.925 & 1.000 \\ \text { XGB } & 0.245 & 0.391 & 0.653 & 0.016 & 1.338 & 0.933 & 2.364\end{array}$

\section{Malawi}

$\begin{array}{cccccccc}\text { Elastic Net } & 0.164 & 0.301 & 0.582 & 0.004 & 1.086 & 0.931 & 1.508 \\ \text { Ensemble } & 0.211 & 0.350 & 0.619 & 0.009 & 1.238 & 0.933 & 1.937 \\ \text { KRLS } & 0.202 & 0.321 & 0.594 & 0.006 & 1.153 & 0.933 & 1.857 \\ \text { Random Forest } & 0.204 & 0.310 & 0.600 & 0.017 & 1.910 & 0.933 & 1.878 \\ \text { Wealth } & 0.109 & 0.215 & 0.533 & 0.063 & 1.456 & 0.929 & 1.000\end{array}$


medRxiv preprint doi: https://doi.org/10.1101/2021.07.20.21260818; this version posted July 23, 2021. The copyright holder for this preprint (which was not certified by peer review) is the author/funder, who has granted medRxiv a license to display the preprint in perpetuity.

All rights reserved. No reuse allowed without permission.

Table 8: Detailed Results by Country (continued)

\begin{tabular}{cccccccc}
\hline Algorithm & Recall 10 & Recall 20 & ROC & MRD & MRR & F1 & Efficiency Gain \\
\hline XGB & 0.184 & 0.319 & 0.594 & 0.007 & 1.175 & 0.932 & 1.692 \\
Mali & & & & & & & \\
Elastic Net & 0.203 & 0.355 & 0.646 & 0.015 & 1.262 & 0.927 & 1.750 \\
$\quad$ Ensemble & 0.245 & 0.371 & 0.666 & 0.025 & 1.410 & 0.929 & 2.114 \\
$\quad$ KRLS & 0.213 & 0.379 & 0.657 & 0.018 & 1.299 & 0.927 & 1.841 \\
Random Forest & 0.221 & 0.347 & 0.634 & 0.050 & 1.742 & 0.928 & 1.909 \\
$\quad$ Wealth & 0.116 & 0.216 & 0.555 & 0.181 & 3.474 & 0.921 & 1.000 \\
XGB & 0.208 & 0.358 & 0.654 & 0.017 & 1.291 & 0.927 & 1.795
\end{tabular}

\section{Mozambique}

$\begin{array}{cccccccc}\text { Elastic Net } & 0.156 & 0.286 & 0.567 & 0.005 & 1.077 & 0.921 & 1.523 \\ \text { Ensemble } & 0.240 & 0.370 & 0.631 & 0.020 & 1.296 & 0.926 & 2.341 \\ \text { KRLS } & 0.207 & 0.323 & 0.591 & 0.010 & 1.148 & 0.924 & 2.023 \\ \text { Random Forest } & 0.244 & 0.365 & 0.625 & 0.051 & 1.676 & 0.927 & 2.386 \\ \text { Wealth } & 0.102 & 0.242 & 0.536 & 0.089 & 2.105 & 0.917 & 1.000 \\ \text { XGB } & 0.179 & 0.326 & 0.598 & 0.015 & 1.228 & 0.922 & 1.750\end{array}$

\section{Niger}

$\begin{array}{cccccccc}\text { Elastic Net } & 0.149 & 0.307 & 0.612 & 0.008 & 1.127 & 0.923 & 1.554 \\ \text { Ensemble } & 0.194 & 0.335 & 0.635 & 0.018 & 1.287 & 0.925 & 2.021 \\ \text { KRLS } & 0.149 & 0.282 & 0.625 & 0.009 & 1.154 & 0.923 & 1.554 \\ \text { Random Forest } & 0.205 & 0.307 & 0.610 & 0.046 & 1.671 & 0.927 & 2.131 \\ \text { Wealth } & 0.096 & 0.196 & 0.532 & 0.158 & 2.954 & 0.919 & 1.000 \\ \text { XGB } & 0.160 & 0.286 & 0.607 & 0.009 & 1.147 & 0.924 & 1.665\end{array}$


medRxiv preprint doi: https://doi.org/10.1101/2021.07.20.21260818; this version posted July 23, 2021. The copyright holder for this preprint (which was not certified by peer review) is the author/funder, who has granted medRxiv a license to display the preprint in perpetuity.

All rights reserved. No reuse allowed without permission.

Table 8: Detailed Results by Country (continued)

\section{Algorithm $\quad$ Recall 10 Recall 20 ROC $\quad$ MRD $\quad$ MRR $\quad$ F1 Efficiency Gain}

\section{Nigeria}

$\begin{array}{cccccccc}\text { Elastic Net } & 0.173 & 0.338 & 0.621 & 0.012 & 1.171 & 0.920 & 1.401 \\ \text { Ensemble } & 0.199 & 0.339 & 0.634 & 0.017 & 1.227 & 0.922 & 1.604 \\ \text { KRLS } & 0.186 & 0.332 & 0.623 & 0.012 & 1.164 & 0.921 & 1.502 \\ \text { Random Forest } & 0.175 & 0.294 & 0.603 & 0.031 & 1.375 & 0.920 & 1.413 \\ \text { Wealth } & 0.124 & 0.251 & 0.568 & 0.223 & 1.934 & 0.916 & 1.000 \\ \text { XGB } & 0.189 & 0.342 & 0.622 & 0.012 & 1.175 & 0.921 & 1.526\end{array}$

\section{Rwanda}

$\begin{array}{cccccccc}\text { Elastic Net } & 0.164 & 0.357 & 0.623 & 0.004 & 1.114 & 0.936 & 1.103 \\ \text { Ensemble } & 0.186 & 0.320 & 0.618 & 0.005 & 1.172 & 0.936 & 1.251 \\ \text { KRLS } & 0.142 & 0.312 & 0.609 & 0.003 & 1.099 & 0.935 & 0.952 \\ \text { Random Forest } & 0.207 & 0.296 & 0.578 & 0.010 & 1.517 & 0.938 & 1.387 \\ \text { Wealth } & 0.149 & 0.259 & 0.561 & 0.196 & 5.841 & 0.936 & 1.000 \\ \text { XGB } & 0.126 & 0.266 & 0.556 & 0.003 & 1.090 & 0.935 & 0.845\end{array}$

\section{Senegal}

$\begin{array}{cccccccc}\text { Elastic Net } & 0.149 & 0.299 & 0.580 & 0.004 & 1.101 & 0.930 & 1.773 \\ \text { Ensemble } & 0.224 & 0.339 & 0.609 & 0.033 & 1.810 & 0.933 & 2.660 \\ \text { KRLS } & 0.165 & 0.315 & 0.581 & 0.004 & 1.100 & 0.931 & 1.957 \\ \text { Random Forest } & 0.209 & 0.311 & 0.587 & 0.059 & 2.194 & 0.933 & 2.475 \\ \text { Wealth } & 0.084 & 0.203 & 0.535 & 0.106 & 1.687 & 0.927 & 1.000 \\ \text { XGB } & 0.209 & 0.333 & 0.595 & 0.064 & 3.297 & 0.933 & 2.476\end{array}$

\section{Tanzania}


medRxiv preprint doi: https://doi.org/10.1101/2021.07.20.21260818; this version posted July 23, 2021. The copyright holder for this preprint (which was not certified by peer review) is the author/funder, who has granted medRxiv a license to display the preprint in perpetuity.

All rights reserved. No reuse allowed without permission.

Table 8: Detailed Results by Country (continued)

\begin{tabular}{cccccccc}
\hline Algorithm & Recall 10 & Recall 20 & ROC & MRD & MRR & F1 & Efficiency Gain \\
\hline Elastic Net & 0.121 & 0.226 & 0.561 & 0.002 & 1.054 & 0.930 & 1.397 \\
Ensemble & 0.188 & 0.262 & 0.582 & 0.005 & 1.145 & 0.932 & 2.169 \\
KRLS & 0.144 & 0.254 & 0.549 & 0.004 & 1.098 & 0.931 & 1.667 \\
Random Forest & 0.180 & 0.309 & 0.584 & 0.011 & 1.459 & 0.932 & 2.080 \\
Wealth & 0.086 & 0.161 & 0.545 & -0.128 & 0.251 & 0.928 & 1.000 \\
XGB & 0.164 & 0.285 & 0.560 & 0.005 & 1.110 & 0.932 & 1.895
\end{tabular}

Uganda

$\begin{array}{cccccccc}\text { Elastic Net } & 0.145 & 0.240 & 0.536 & 0.001 & 1.019 & 0.930 & 1.402 \\ \text { Ensemble } & 0.178 & 0.307 & 0.579 & 0.002 & 1.059 & 0.932 & 1.725 \\ \text { KRLS } & 0.134 & 0.237 & 0.535 & 0.001 & 1.027 & 0.930 & 1.299 \\ \text { Random Forest } & 0.167 & 0.278 & 0.554 & 0.001 & 1.620 & 0.931 & 1.624 \\ \text { Wealth } & 0.103 & 0.224 & 0.537 & -0.021 & 0.919 & 0.929 & 1.000 \\ \text { XGB } & 0.157 & 0.294 & 0.576 & 0.004 & 1.104 & 0.931 & 1.526\end{array}$

\section{Zambia}

$\begin{array}{cccccccc}\text { Elastic Net } & 0.150 & 0.310 & 0.579 & 0.005 & 1.099 & 0.928 & 1.875 \\ \text { Ensemble } & 0.209 & 0.354 & 0.617 & 0.008 & 1.190 & 0.930 & 2.619 \\ \text { KRLS } & 0.186 & 0.333 & 0.588 & 0.005 & 1.098 & 0.930 & 2.327 \\ \text { Random Forest } & 0.209 & 0.300 & 0.598 & 0.016 & 1.588 & 0.931 & 2.618 \\ \text { Wealth } & 0.080 & 0.163 & 0.529 & -0.009 & 0.990 & 0.924 & 1.000 \\ \text { XGB } & 0.194 & 0.320 & 0.595 & 0.008 & 1.160 & 0.930 & 2.422\end{array}$

\section{Zimbabwe}

Elastic Net

0.143

0.308

0.576

0.005

1.095

0.926

1.176 
medRxiv preprint doi: https://doi.org/10.1101/2021.07.20.21260818; this version posted July 23, 2021. The copyright holder for this preprint (which was not certified by peer review) is the author/funder, who has granted medRxiv a license to display the preprint in perpetuity.

All rights reserved. No reuse allowed without permission.

Table 8: Detailed Results by Country (continued)

\begin{tabular}{cccccccc}
\hline Algorithm & Recall 10 & Recall 20 & ROC & MRD & MRR & F1 & Efficiency Gain \\
\hline Ensemble & 0.190 & 0.333 & 0.642 & 0.010 & 1.216 & 0.929 & 1.569 \\
KRLS & 0.154 & 0.292 & 0.600 & 0.005 & 1.108 & 0.927 & 1.265 \\
Random Forest & 0.201 & 0.338 & 0.629 & 0.019 & 1.726 & 0.930 & 1.653 \\
Wealth & 0.121 & 0.228 & 0.570 & 2.029 & -170.854 & 0.925 & 1.000 \\
XGB & 0.175 & 0.318 & 0.588 & 0.009 & 1.184 & 0.928 & 1.441 \\
\hline
\end{tabular}

\section{B.2 Angola}

This sample was taken in the year 2015. There are 10829 observations in the dataset, and 8664 observations were used for the training set. Also, 195 observations were removed due to missing values. Finally, there were 472 deaths for the full dataset, and 378 deaths in the training set. 


\section{B.2.1 Variables Used}

Table 9: Variables included in the model for Angola

\begin{tabular}{|c|c|}
\hline Variable & Type \\
\hline mortality.under $12 \mathrm{~m}$ & categorical \\
\hline pregtermin & categorical \\
\hline hheadagehh & numeric \\
\hline urban & categorical \\
\hline kidsex & categorical \\
\hline hheadsex & categorical \\
\hline edyrtotal & numeric \\
\hline drinkwtr_new & categorical \\
\hline floor_new & categorical \\
\hline maternal_age_month & numeric \\
\hline cookfuel_new & categorical \\
\hline toilettype_new & categorical \\
\hline religion_new & categorical \\
\hline bednetnumlog & numeric \\
\hline prev_death & categorical \\
\hline prev_death_full & categorical \\
\hline kidbordlog & numeric \\
\hline kidbirthmo_sin & numeric \\
\hline kidbirthmo_cos & numeric \\
\hline geo_ao2015 & categorical \\
\hline wealthp & numeric \\
\hline wealthp2 & numeric \\
\hline
\end{tabular}




\section{B.2.2 Mortality Breakdown by Wealth Quintile}

\section{B.2.3 Optimal Parameters}

These are the algorithm parameters selected after cross-validation:

Table 10: Optimal Parameters for the Elastic Net algorithm

\begin{tabular}{|c|c|}
\hline alpha & lambda \\
\hline 0.55 & 0.0040056 \\
\hline
\end{tabular}

Table 11: Optimal Parameters for the Random Forest algorithm

\begin{tabular}{|c|}
\hline mtry \\
\hline 2 \\
\hline
\end{tabular}

Table 12: Optimal Parameters for the XGB algorithm

\begin{tabular}{|c|c|c|c|c|c|c|}
\hline nrounds & max_depth & eta & gamma & colsample_bytree & min_child_weight & subsample \\
\hline 50 & 1 & 0.3 & 0 & 0.8 & 1 & 0.625 \\
\hline
\end{tabular}

Table 13: Optimal Parameters for the KRLS algorithm

\begin{tabular}{|c|c|c|c|}
\hline loss & epsilon & b & lambda \\
\hline logistic & 0.005 & 80 & $4.85 \mathrm{e}-05$ \\
\hline
\end{tabular}


medRxiv preprint doi: https://doi.org/10.1101/2021.07.20.21260818; this version posted July 23, 2021. The copyright holder for this preprint (which was not certified by peer review) is the author/funder, who has granted medRxiv a license to display the preprint in perpetuity.

\section{B.2.4 Table of Results}

Table 14: Manual Cross-Validation Results

\begin{tabular}{|c|c|c|c|c|c|c|c|c|c|}
\hline Algorithm & Recall 10 & Recall 20 & ROC & AUC & MRD & MRR & MSE & F1 & Efficiency Gain \\
\hline Ensemble & 0.244 & 0.424 & 0.683 & 0.099 & 0.014 & 1.399 & 0.041 & 0.934 & 2.300 \\
\hline KRLS & 0.236 & 0.384 & 0.669 & 0.085 & 0.016 & 1.379 & 0.041 & 0.934 & 2.227 \\
\hline Elastic Net & 0.212 & 0.394 & 0.664 & 0.083 & 0.012 & 1.276 & 0.041 & 0.933 & 2.001 \\
\hline Random Forest & 0.214 & 0.368 & 0.641 & 0.102 & 0.013 & 1.904 & 0.042 & 0.933 & 2.024 \\
\hline Wealth & 0.106 & 0.241 & 0.560 & 0.055 & 0.207 & 15.020 & 0.921 & 0.928 & 1.000 \\
\hline XGB & 0.225 & 0.397 & 0.666 & 0.087 & 0.016 & 1.379 & 0.041 & 0.934 & 2.124 \\
\hline
\end{tabular}

Table 15: Distribution of individuals in the top risk decile of each algorithm among each wealth decile

\begin{tabular}{l|r|r|r|r|c}
\hline Wealth Decile & Elastic Net & Ensemble & KRLS & Random Forest & XGB \\
\hline 1 & 0.153 & 0.166 & 0.173 & 0.144 & 0.153 \\
\hline 2 & 0.163 & 0.177 & 0.186 & 0.110 & 0.195 \\
\hline 3 & 0.140 & 0.143 & 0.152 & 0.097 & 0.131 \\
\hline 4 & 0.134 & 0.149 & 0.140 & 0.127 & 0.158 \\
\hline 5 & 0.115 & 0.126 & 0.115 & 0.150 & 0.134 \\
\hline 6 & 0.108 & 0.087 & 0.090 & 0.114 & 0.076 \\
\hline 7 & 0.067 & 0.060 & 0.053 & 0.064 & 0.053 \\
\hline 8 & 0.050 & 0.040 & 0.040 & 0.060 & 0.036 \\
\hline 9 & 0.042 & 0.033 & 0.030 & 0.066 & 0.031 \\
\hline 10 & 0.027 & 0.026 & 0.020 & 0.067 & 0.030 \\
\hline
\end{tabular}

\section{B.2.5 Performance Plots}

Below are various plots showing the performance for each model considered in Angola. 
medRxiv preprint doi: https://doi.org/10.1101/2021.07.20.21260818; this version posted July 23, 2021. The copyright holder for this preprint (which was not certified by peer review) is the author/funder, who has granted medRxiv a license to display the preprint in perpetuity. All rights reserved. No reuse allowed without permission.

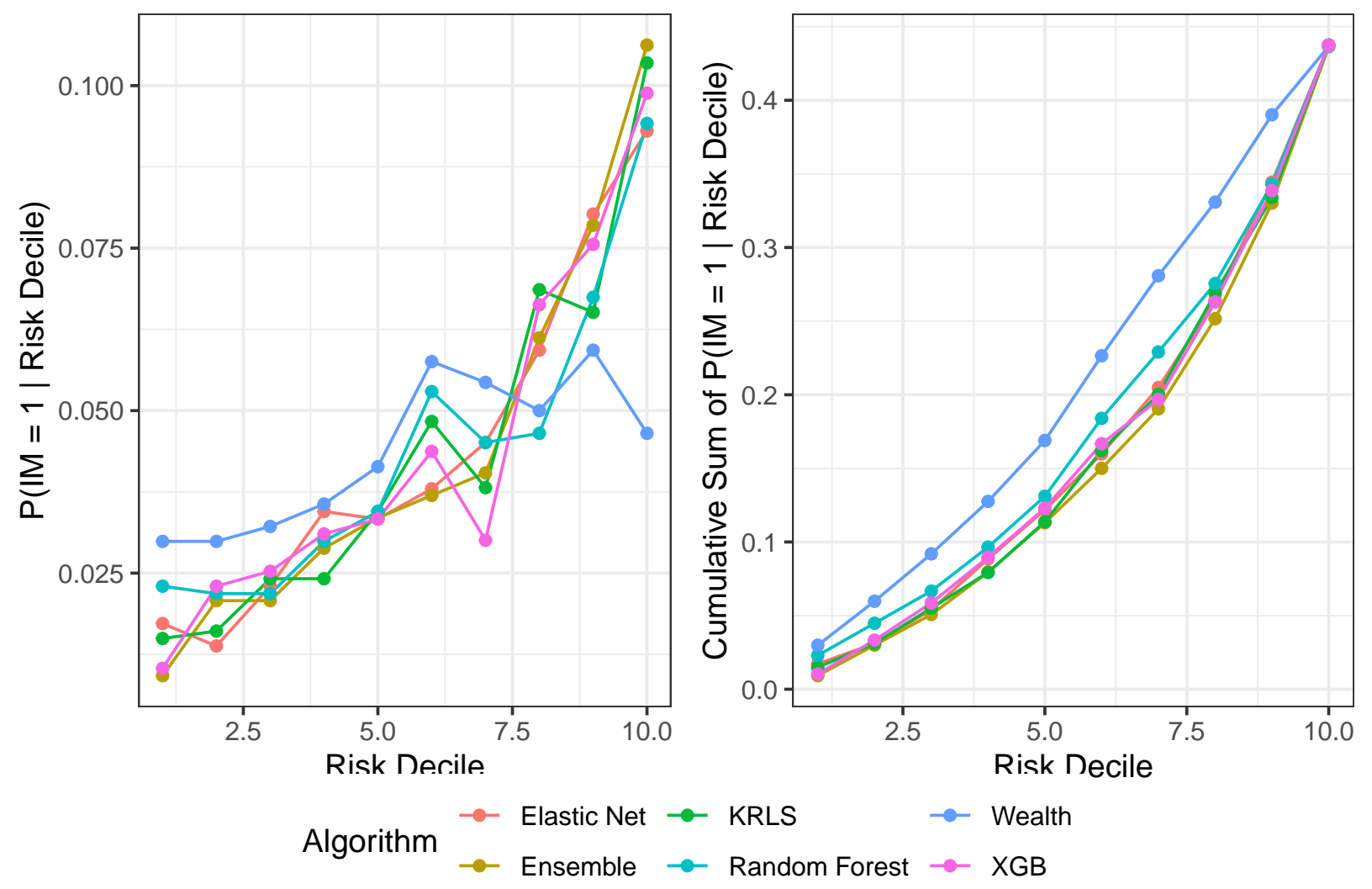

Figure 1: Probability of a mortality given that the observation is in a particular risk decile 
medRxiv preprint doi: https://doi.org/10.1101/2021.07.20.21260818; this version posted July 23, 2021. The copyright holder for this preprint (which was not certified by peer review) is the author/funder, who has granted medRxiv a license to display the preprint in perpetuity. All rights reserved. No reuse allowed without permission.
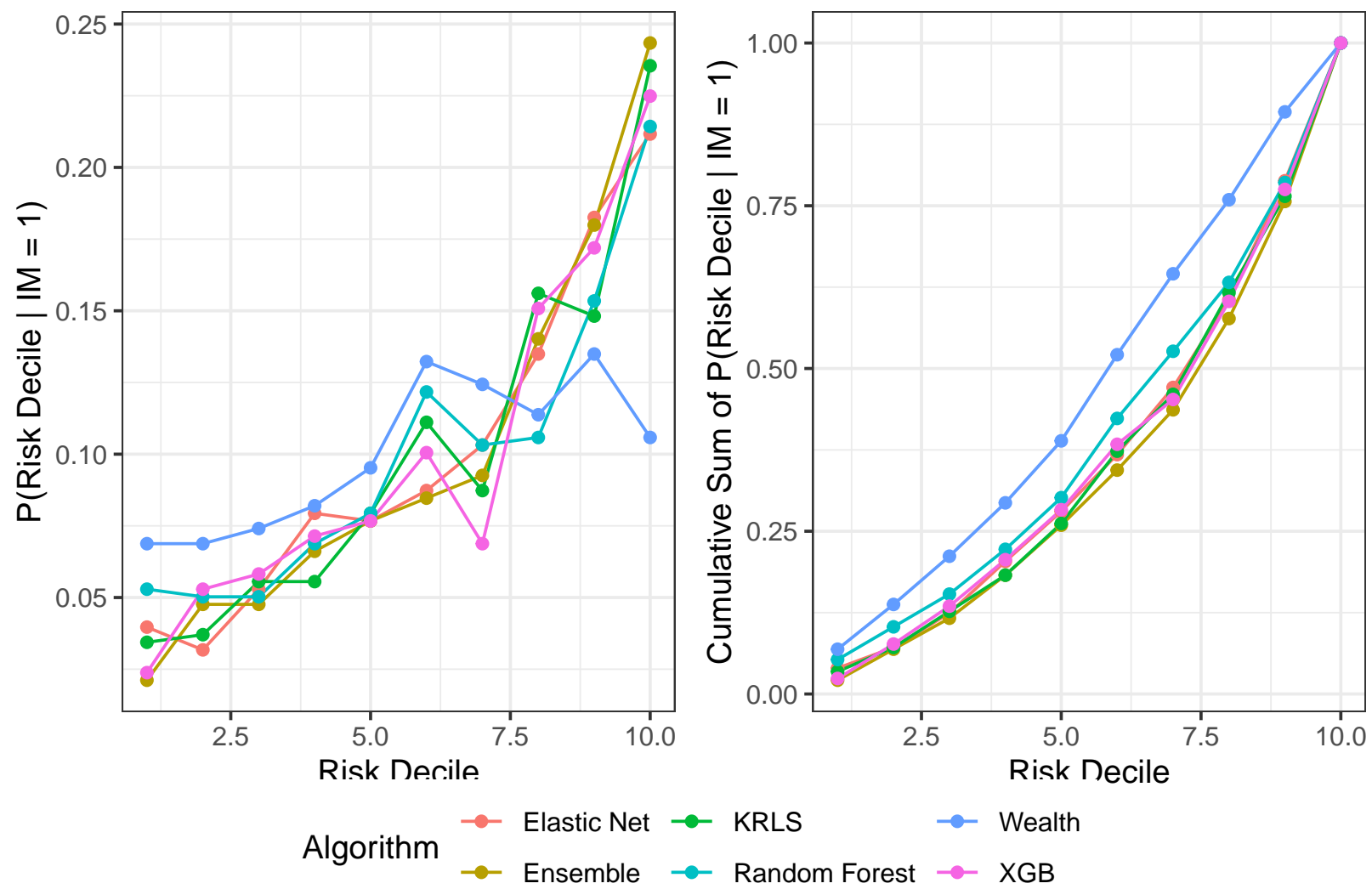

Figure 2: Probability of membership in a particular risk decile given a mortality 


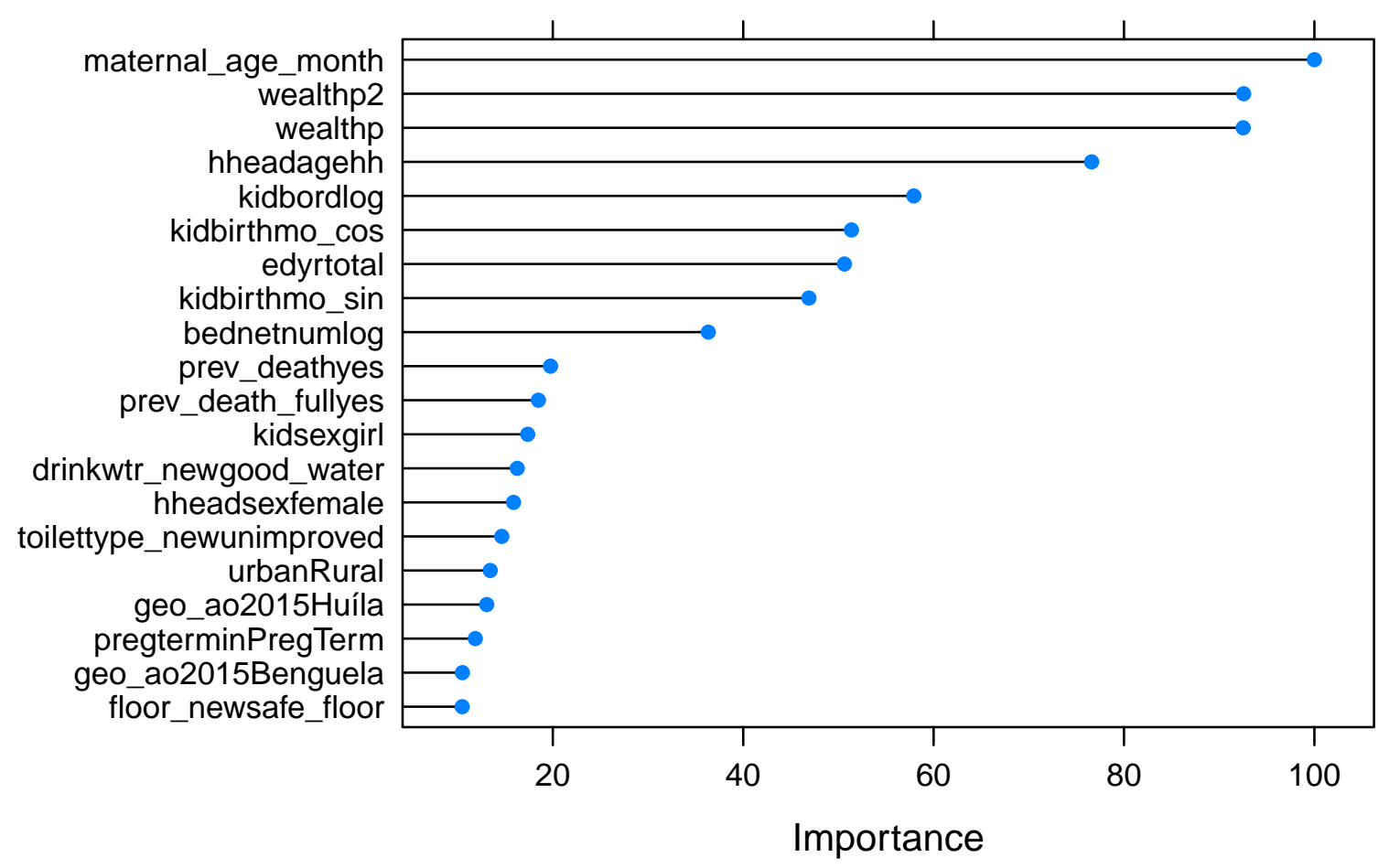

Figure 3: Variable Importance Plot generated by the Random Forest model

\section{B.2.6 Variable Importance Plot}

In this section we will show some measures to give some indication of the important variables necessary for predicting infant mortality. 
medRxiv preprint doi: https://doi.org/10.1101/2021.07.20.21260818; this version posted July 23, 2021. The copyright holder for this preprint (which was not certified by peer review) is the author/funder, who has granted medRxiv a license to display the preprint in perpetuity.

All rights reserved. No reuse allowed without permission.

\section{B.2.7 Elastic Net Coefficients}

Table 16: Standardized coefficients from the optimal elastic net model after 10-fold crossvalidation.

\begin{tabular}{|c|c|c|}
\hline & Coefficients & Standardized Coefficients \\
\hline (Intercept) & -2.5213 & -2.5213 \\
\hline pregterminPregTerm & - & - \\
\hline hheadagehh & $6 e-04$ & 0.0073 \\
\hline urbanRural & - & - \\
\hline kidsexgirl & -0.2118 & -0.1059 \\
\hline hheadsexfemale & - & - \\
\hline edyrtotal & - & - \\
\hline drinkwtr_newgood_water & 0.0334 & 0.016 \\
\hline floor_newsafe_floor & -0.0109 & -0.0053 \\
\hline maternal_age_month & -0.002 & -0.1704 \\
\hline cookfuel_newclean_fuel & - & - \\
\hline toilettype_newpit & - & - \\
\hline toilettype_newunimproved & - & - \\
\hline religion_newChristian & - & - \\
\hline religion_newHindu & - & - \\
\hline religion_newMuslim & - & - \\
\hline religion_newOther & - & - \\
\hline bednetnumlog & - & - \\
\hline prev_deathyes & 0.6642 & 0.2278 \\
\hline prev_death_fullyes & 0.3219 & 0.1231 \\
\hline kidbordlog & - & - \\
\hline kidbirthmo_sin & - & - \\
\hline kidbirthmo_cos & - & - \\
\hline geo_ao2015Zaire & -0.0145 & -0.0032 \\
\hline geo_ao2015Uíge & - & - \\
\hline geo_ao2015Luanda & - & - \\
\hline geo_ao2015Cuanza Norte & - & - \\
\hline geo_ao2015Cuanza Sul & 0.0993 & 0.0228 \\
\hline geo_ao2015Malanje & - & - \\
\hline geo_ao2015Lunda Norte & 0.223 & 0.0504 \\
\hline geo_ao2015Benguela & 0.3073 & 0.0747 \\
\hline geo_ao2015Huambo & 0.1606 & 0.0405 \\
\hline geo_ao2015Bié & - & - \\
\hline geo_ao2015Moxico & -0.5247 & -0.0982 \\
\hline geo_ao2015Cuando Cubango & - & - \\
\hline geo_ao2015Namibe & - & - \\
\hline geo_ao2015Huíla & 0.389 & 0.0965 \\
\hline geo_ao2015Cunene & - & - \\
\hline geo_ao2015Lunda Sul & - & - \\
\hline
\end{tabular}


medRxiv preprint doi: https://doi.org/10.1101/2021.07.20.21260818; this version posted July 23, 2021. The copyright holder for this preprint (which was not certified by peer review) is the author/funder, who has granted medRxiv a license to display the preprint in perpetuity.

All rights reserved. No reuse allowed without permission.

Table 16: Standardized coefficients from the optimal elastic net model after 10-fold crossvalidation. (continued)

\begin{tabular}{|l|c|c|}
\hline & Coefficients & Standardized Coefficients \\
\hline geo_ao2015Bengo & -0.2043 & -0.041 \\
\hline wealthp & - & - \\
\hline wealthp2 & -0.4321 & -0.129 \\
\hline
\end{tabular}


medRxiv preprint doi: https://doi.org/10.1101/2021.07.20.21260818; this version posted July 23, 2021. The copyright holder for this preprint (which was not certified by peer review) is the author/funder, who has granted medRxiv a license to display the preprint in perpetuity.

All rights reserved. No reuse allowed without permission.

\section{B.3 Benin}

This sample was taken in the year 2011. There are 10199 observations in the dataset, and 8160 observations were used for the training set. Also, 291 observations were removed due to missing values. Finally, there were 471 deaths for the full dataset, and 377 deaths in the training set. 
medRxiv preprint doi: https://doi.org/10.1101/2021.07.20.21260818; this version posted July 23, 2021. The copyright holder for this preprint (which was not certified by peer review) is the author/funder, who has granted medRxiv a license to display the preprint in perpetuity.

All rights reserved. No reuse allowed without permission. 


\section{B.3.1 Variables Used}

Table 17: Variables included in the model for Benin

\begin{tabular}{|c|c|}
\hline Variable & Type \\
\hline mortality.under $12 \mathrm{~m}$ & categorical \\
\hline pregtermin & categorical \\
\hline agefrstmar & numeric \\
\hline hheadagehh & numeric \\
\hline urban & categorical \\
\hline kidsex & categorical \\
\hline hheadsex & categorical \\
\hline edyrtotal & numeric \\
\hline drinkwtr_new & categorical \\
\hline floor_new & categorical \\
\hline maternal_age_month & numeric \\
\hline cookfuel_new & categorical \\
\hline toilettype_new & categorical \\
\hline religion_new & categorical \\
\hline bednetnumlog & numeric \\
\hline prev_death & categorical \\
\hline prev_death_full & categorical \\
\hline malaria_new & numeric \\
\hline kidbordlog & numeric \\
\hline kidbirthmo_sin & numeric \\
\hline kidbirthmo_cos & numeric \\
\hline geo_bj2011 & categorical \\
\hline wealthp & numeric \\
\hline wealthp2 & numeric \\
\hline
\end{tabular}




\section{B.3.2 Mortality Breakdown by Wealth Quintile}

\section{B.3.3 Optimal Parameters}

These are the algorithm parameters selected after cross-validation:

Table 18: Optimal Parameters for the Elastic Net algorithm

\begin{tabular}{|c|c|}
\hline alpha & lambda \\
\hline 0.1 & 0.0063933 \\
\hline
\end{tabular}

Table 19: Optimal Parameters for the Random Forest algorithm

\begin{tabular}{|c|}
\hline mtry \\
\hline 2 \\
\hline
\end{tabular}

Table 20: Optimal Parameters for the XGB algorithm

\begin{tabular}{|c|c|c|c|c|c|c|}
\hline nrounds & max_depth & eta & gamma & colsample_bytree & min_child_weight & subsample \\
\hline 50 & 1 & 0.3 & 0 & 0.8 & 1 & 0.75 \\
\hline
\end{tabular}

Table 21: Optimal Parameters for the KRLS algorithm

\begin{tabular}{|c|c|c|c|}
\hline loss & epsilon & $\mathbf{b}$ & lambda \\
\hline logistic & 0.005 & 72 & 0.0001239 \\
\hline
\end{tabular}


medRxiv preprint doi: https://doi.org/10.1101/2021.07.20.21260818; this version posted July 23, 2021. The copyright holder for this preprint (which was not certified by peer review) is the author/funder, who has granted medRxiv a license to display the preprint in perpetuity.

\section{B.3.4 Table of Results}

Table 22: Manual Cross-Validation Results

\begin{tabular}{|c|c|c|c|c|c|c|c|c|c|}
\hline Algorithm & Recall 10 & Recall 20 & ROC & AUC & MRD & MRR & MSE & F1 & Efficiency Gain \\
\hline Ensemble & 0.287 & 0.475 & 0.685 & 0.109 & 0.023 & 1.590 & 0.043 & 0.935 & 2.299 \\
\hline KRLS & 0.292 & 0.449 & 0.674 & 0.098 & 0.021 & 1.475 & 0.043 & 0.936 & 2.338 \\
\hline Elastic Net & 0.284 & 0.454 & 0.678 & 0.106 & 0.026 & 1.568 & 0.043 & 0.936 & 2.276 \\
\hline Random Forest & 0.260 & 0.411 & 0.662 & 0.123 & 0.022 & 2.046 & 0.044 & 0.935 & 2.083 \\
\hline Wealth & 0.125 & 0.242 & 0.537 & 0.061 & 0.118 & 2.244 & 0.894 & 0.928 & 1.000 \\
\hline XGB & 0.278 & 0.446 & 0.666 & 0.097 & 0.024 & 1.518 & 0.043 & 0.935 & 2.233 \\
\hline
\end{tabular}

Table 23: Distribution of individuals in the top risk decile of each algorithm among each wealth decile

\begin{tabular}{l|r|r|r|r|c}
\hline Wealth Decile & Elastic Net & Ensemble & KRLS & Random Forest & XGB \\
\hline 1 & 0.141 & 0.154 & 0.147 & 0.165 & 0.146 \\
\hline 2 & 0.140 & 0.142 & 0.138 & 0.124 & 0.152 \\
\hline 3 & 0.142 & 0.147 & 0.143 & 0.110 & 0.137 \\
\hline 4 & 0.147 & 0.141 & 0.147 & 0.102 & 0.140 \\
\hline 5 & 0.077 & 0.078 & 0.081 & 0.088 & 0.077 \\
\hline 6 & 0.094 & 0.099 & 0.099 & 0.094 & 0.098 \\
\hline 7 & 0.091 & 0.085 & 0.086 & 0.067 & 0.072 \\
\hline 8 & 0.068 & 0.059 & 0.067 & 0.068 & 0.069 \\
\hline 9 & 0.062 & 0.062 & 0.059 & 0.098 & 0.068 \\
\hline 10 & 0.038 & 0.040 & 0.031 & 0.083 & 0.041 \\
\hline
\end{tabular}

\section{B.3.5 Performance Plots}

Below are various plots showing the performance for each model considered in Benin. 
medRxiv preprint doi: https://doi.org/10.1101/2021.07.20.21260818; this version posted July 23, 2021. The copyright holder for this preprint (which was not certified by peer review) is the author/funder, who has granted medRxiv a license to display the preprint in perpetuity. All rights reserved. No reuse allowed without permission.

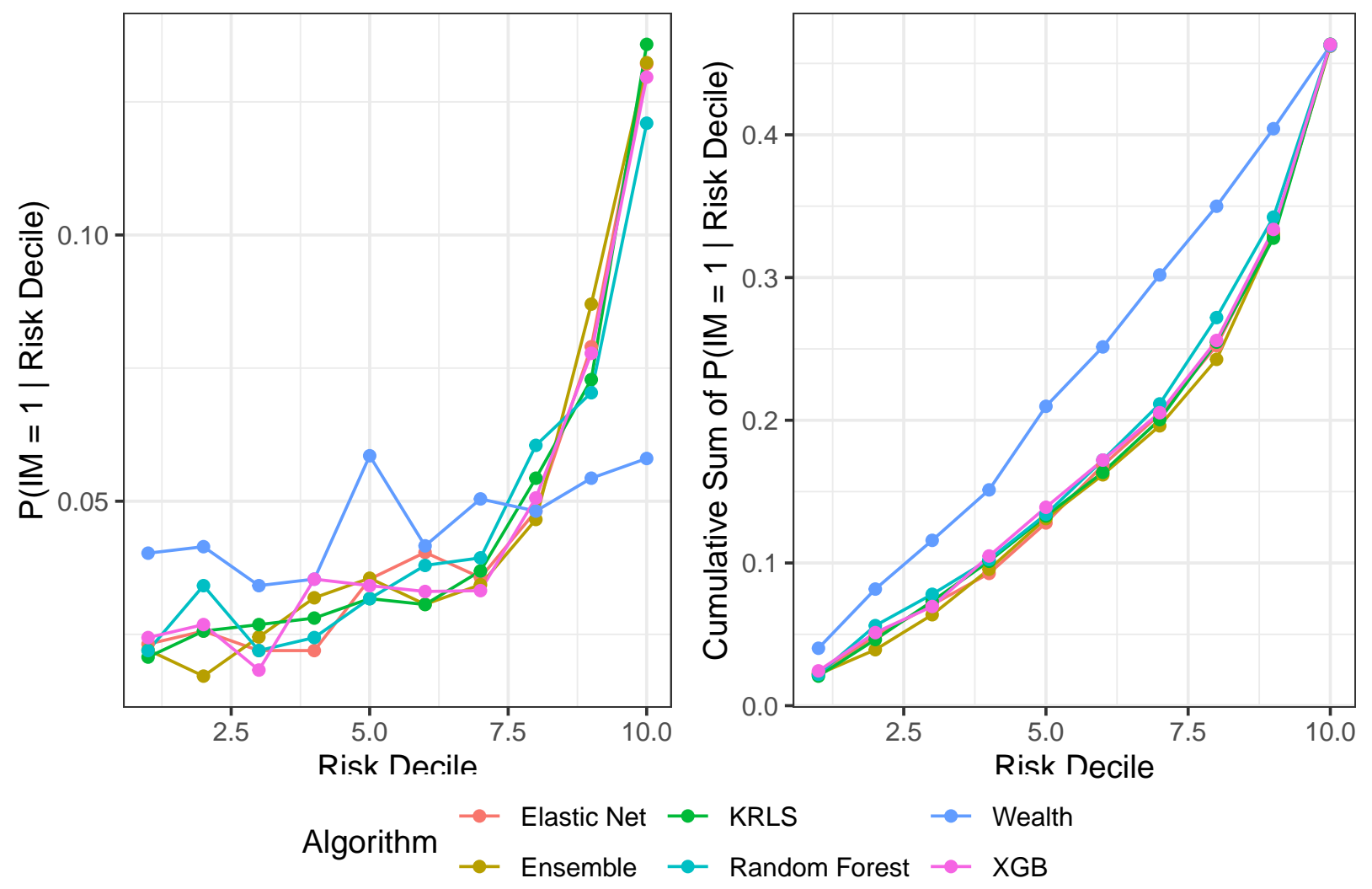

Figure 4: Probability of a mortality given that the observation is in a particular risk decile 

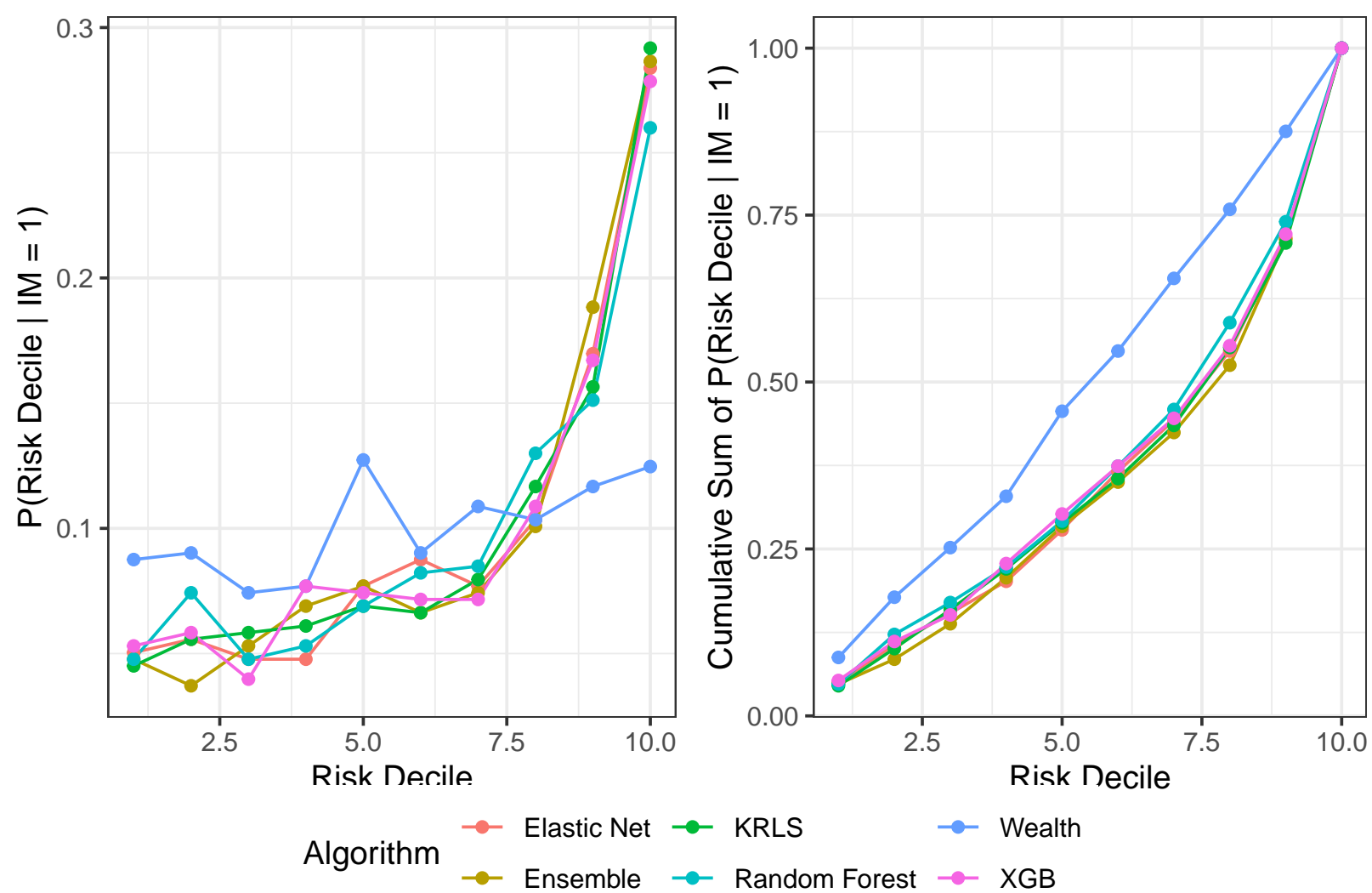

Figure 5: Probability of membership in a particular risk decile given a mortality 


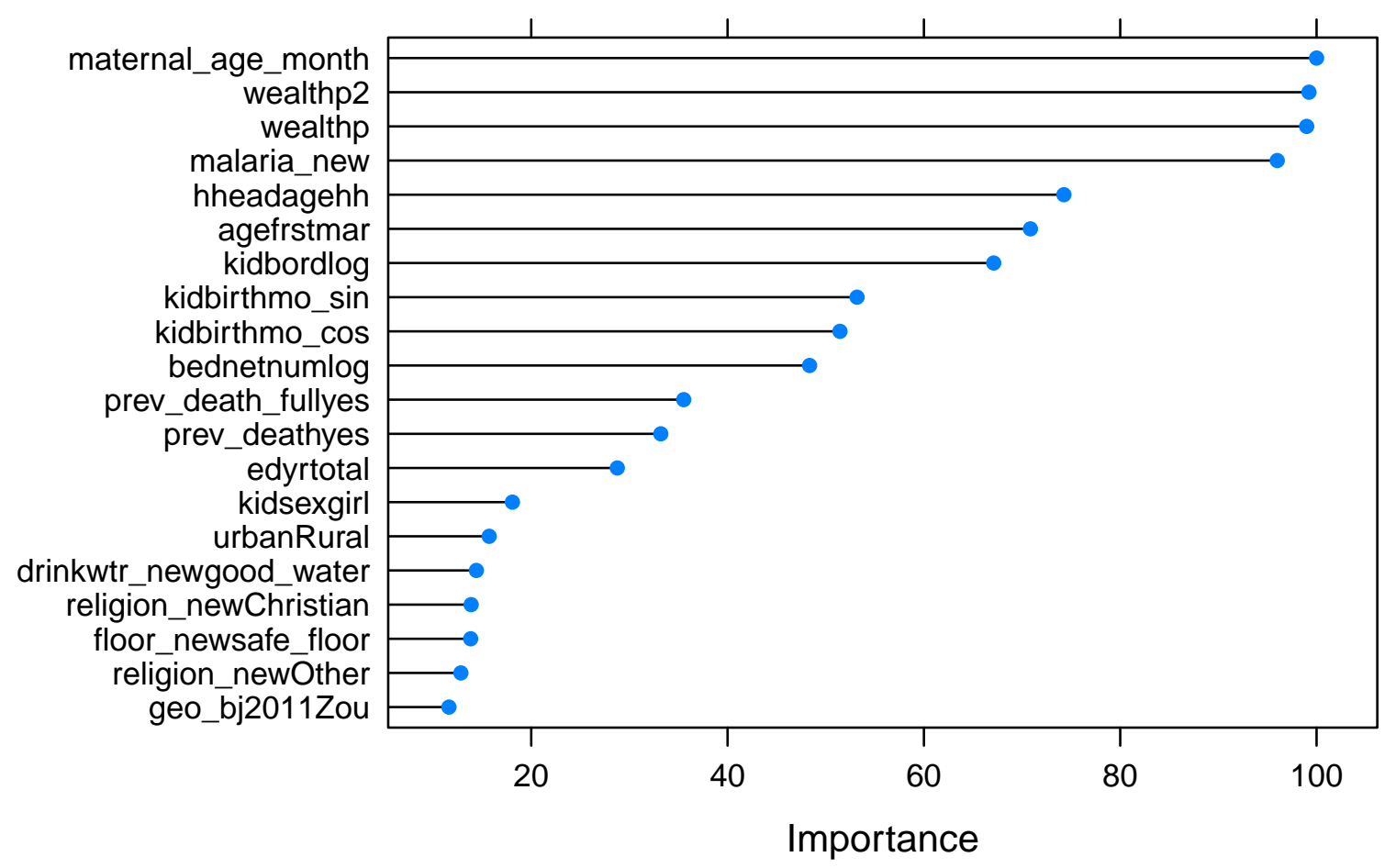

Figure 6: Variable Importance Plot generated by the Random Forest model

\section{B.3.6 Variable Importance Plot}

In this section we will show some measures to give some indication of the important variables necessary for predicting infant mortality. 
medRxiv preprint doi: https://doi.org/10.1101/2021.07.20.21260818; this version posted July 23, 2021. The copyright holder for this preprint (which was not certified by peer review) is the author/funder, who has granted medRxiv a license to display the preprint in perpetuity.

All rights reserved. No reuse allowed without permission.

\section{B.3.7 Elastic Net Coefficients}

Table 24: Standardized coefficients from the optimal elastic net model after 10-fold crossvalidation.

\begin{tabular}{|c|c|c|}
\hline & Coefficients & Standardized Coefficients \\
\hline (Intercept) & -2.2806 & -2.2806 \\
\hline pregterminPregTerm & -0.2594 & -0.0625 \\
\hline agefrstmar & -0.0112 & -0.0498 \\
\hline hheadagehh & $-8 \mathrm{e}-04$ & -0.009 \\
\hline urbanRural & -0.123 & -0.0591 \\
\hline kidsexgirl & -0.1702 & -0.0851 \\
\hline hheadsexfemale & -0.1426 & -0.0448 \\
\hline edyrtotal & -0.0077 & -0.0239 \\
\hline drinkwtr_newgood_water & 0.0945 & 0.0412 \\
\hline floor_newsafe_floor & -0.1028 & -0.0509 \\
\hline maternal_age_month & -0.0018 & -0.1317 \\
\hline cookfuel_newclean_fuel & - & - \\
\hline toilettype_newpit & -0.1219 & -0.056 \\
\hline toilettype_newunimproved & - & - \\
\hline religion_newChristian & - & - \\
\hline religion_newHindu & - & - \\
\hline religion_newMuslim & -0.0057 & -0.0025 \\
\hline religion_newOther & 0.0498 & 0.0207 \\
\hline bednetnumlog & -0.0252 & -0.0128 \\
\hline prev_deathyes & 0.5222 & 0.1642 \\
\hline prev_death_fullyes & 0.8644 & 0.3144 \\
\hline malaria_new & $9 \mathrm{e}-04$ & $1 \mathrm{e}-04$ \\
\hline kidbordlog & 0.1375 & 0.0896 \\
\hline kidbirthmo_sin & 0.0954 & 0.0682 \\
\hline kidbirthmo_cos & -0.0308 & -0.0216 \\
\hline geo_bj2011Atacora & -0.3459 & -0.1056 \\
\hline geo_bj2011Atlantique & 0.1161 & 0.0376 \\
\hline geo_bj2011Borgou & -0.2252 & -0.0622 \\
\hline geo_bj2011Collines & -0.0098 & -0.0025 \\
\hline geo_bj2011Couffo & - & - \\
\hline geo_bj2011Donga & 0.0855 & 0.0195 \\
\hline geo_bj2011Littoral & 0.4842 & 0.1308 \\
\hline geo_bj2011Mono & -0.3927 & -0.0926 \\
\hline geo_bj2011Ouémé & -0.3573 & -0.1126 \\
\hline geo_bj2011Plateau & -0.4086 & -0.1009 \\
\hline geo_bj2011Zou & 0.0573 & 0.017 \\
\hline wealthp & -0.1813 & -0.0524 \\
\hline wealthp2 & -0.0293 & -0.0088 \\
\hline
\end{tabular}


medRxiv preprint doi: https://doi.org/10.1101/2021.07.20.21260818; this version posted July 23, 2021. The copyright holder for this preprint (which was not certified by peer review) is the author/funder, who has granted medRxiv a license to display the preprint in perpetuity.

All rights reserved. No reuse allowed without permission.

\section{B.4 Burkina Faso}

This sample was taken in the year 2010. There are 10905 observations in the dataset, and 8725 observations were used for the training set. Also, 823 observations were removed due to missing values. Finally, there were 752 deaths for the full dataset, and 602 deaths in the training set. 
medRxiv preprint doi: https://doi.org/10.1101/2021.07.20.21260818; this version posted July 23, 2021. The copyright holder for this preprint (which was not certified by peer review) is the author/funder, who has granted medRxiv a license to display the preprint in perpetuity.

All rights reserved. No reuse allowed without permission. 


\section{B.4.1 Variables Used}

Table 25: Variables included in the model for Burkina Faso

\begin{tabular}{|c|c|}
\hline Variable & Type \\
\hline mortality.under $12 \mathrm{~m}$ & categorical \\
\hline pregtermin & categorical \\
\hline agefrstmar & numeric \\
\hline hheadagehh & numeric \\
\hline urban & categorical \\
\hline kidsex & categorical \\
\hline hheadsex & categorical \\
\hline edyrtotal & numeric \\
\hline drinkwtr_new & categorical \\
\hline floor_new & categorical \\
\hline maternal_age_month & numeric \\
\hline cookfuel_new & categorical \\
\hline toilettype_new & categorical \\
\hline religion_new & categorical \\
\hline bednetnumlog & numeric \\
\hline prev_death & categorical \\
\hline prev_death_full & categorical \\
\hline malaria_new & numeric \\
\hline kidbordlog & numeric \\
\hline kidbirthmo_sin & numeric \\
\hline kidbirthmo_cos & numeric \\
\hline geo_bf2010 & categorical \\
\hline wealthp & numeric \\
\hline wealthp2 & numeric \\
\hline
\end{tabular}




\section{B.4.2 Mortality Breakdown by Wealth Quintile}

\section{B.4.3 Optimal Parameters}

These are the algorithm parameters selected after cross-validation:

Table 26: Optimal Parameters for the Elastic Net algorithm

\begin{tabular}{|c|c|}
\hline alpha & lambda \\
\hline 0.1 & 0.0214685 \\
\hline
\end{tabular}

Table 27: Optimal Parameters for the Random Forest algorithm

\begin{tabular}{|c|}
\hline mtry \\
\hline 2 \\
\hline
\end{tabular}

Table 28: Optimal Parameters for the XGB algorithm

\begin{tabular}{|c|c|c|c|c|c|c|}
\hline nrounds & max_depth & eta & gamma & colsample_bytree & min_child_weight & subsample \\
\hline 50 & 1 & 0.3 & 0 & 0.8 & 1 & 0.875 \\
\hline
\end{tabular}

Table 29: Optimal Parameters for the KRLS algorithm

\begin{tabular}{|c|c|c|c|}
\hline loss & epsilon & $\mathbf{b}$ & lambda \\
\hline logistic & 0.005 & 74 & 0.0001417 \\
\hline
\end{tabular}


medRxiv preprint doi: https://doi.org/10.1101/2021.07.20.21260818; this version posted July 23, 2021. The copyright holder for this preprint (which was not certified by peer review) is the author/funder, who has granted medRxiv a license to display the preprint in perpetuity.

\section{B.4.4 Table of Results}

Table 30: Manual Cross-Validation Results

\begin{tabular}{|c|c|c|c|c|c|c|c|c|c|}
\hline Algorithm & Recall 10 & Recall 20 & ROC & AUC & MRD & MRR & MSE & F1 & Efficiency Gain \\
\hline Ensemble & 0.179 & 0.304 & 0.626 & 0.115 & 0.010 & 1.175 & 0.064 & 0.921 & 2.076 \\
\hline KRLS & 0.145 & 0.297 & 0.611 & 0.111 & 0.010 & 1.152 & 0.064 & 0.919 & 1.673 \\
\hline Elastic Net & 0.161 & 0.307 & 0.608 & 0.111 & 0.008 & 1.120 & 0.064 & 0.920 & 1.866 \\
\hline Random Forest & 0.155 & 0.287 & 0.581 & 0.105 & 0.009 & 1.349 & 0.065 & 0.920 & 1.789 \\
\hline Wealth & 0.086 & 0.234 & 0.553 & 0.077 & 0.147 & 1.958 & 0.720 & 0.914 & 1.000 \\
\hline XGB & 0.159 & 0.306 & 0.620 & 0.112 & 0.013 & 1.188 & 0.064 & 0.920 & 1.845 \\
\hline
\end{tabular}

Table 31: Distribution of individuals in the top risk decile of each algorithm among each wealth decile

\begin{tabular}{l|r|r|r|r|c}
\hline Wealth Decile & Elastic Net & Ensemble & KRLS & Random Forest & XGB \\
\hline 1 & 0.229 & 0.171 & 0.231 & 0.116 & 0.101 \\
\hline 2 & 0.216 & 0.225 & 0.212 & 0.126 & 0.242 \\
\hline 3 & 0.169 & 0.168 & 0.177 & 0.090 & 0.183 \\
\hline 4 & 0.145 & 0.149 & 0.140 & 0.097 & 0.180 \\
\hline 5 & 0.080 & 0.089 & 0.090 & 0.097 & 0.107 \\
\hline 6 & 0.054 & 0.071 & 0.060 & 0.102 & 0.078 \\
\hline 7 & 0.050 & 0.049 & 0.049 & 0.093 & 0.047 \\
\hline 8 & 0.041 & 0.037 & 0.030 & 0.095 & 0.032 \\
\hline 9 & 0.010 & 0.033 & 0.009 & 0.099 & 0.025 \\
\hline 10 & 0.005 & 0.009 & 0.001 & 0.085 & 0.003 \\
\hline
\end{tabular}

\section{B.4.5 Performance Plots}

Below are various plots showing the performance for each model considered in Burkina Faso. 
medRxiv preprint doi: https://doi.org/10.1101/2021.07.20.21260818; this version posted July 23, 2021. The copyright holder for this preprint (which was not certified by peer review) is the author/funder, who has granted medRxiv a license to display the preprint in perpetuity. All rights reserved. No reuse allowed without permission.
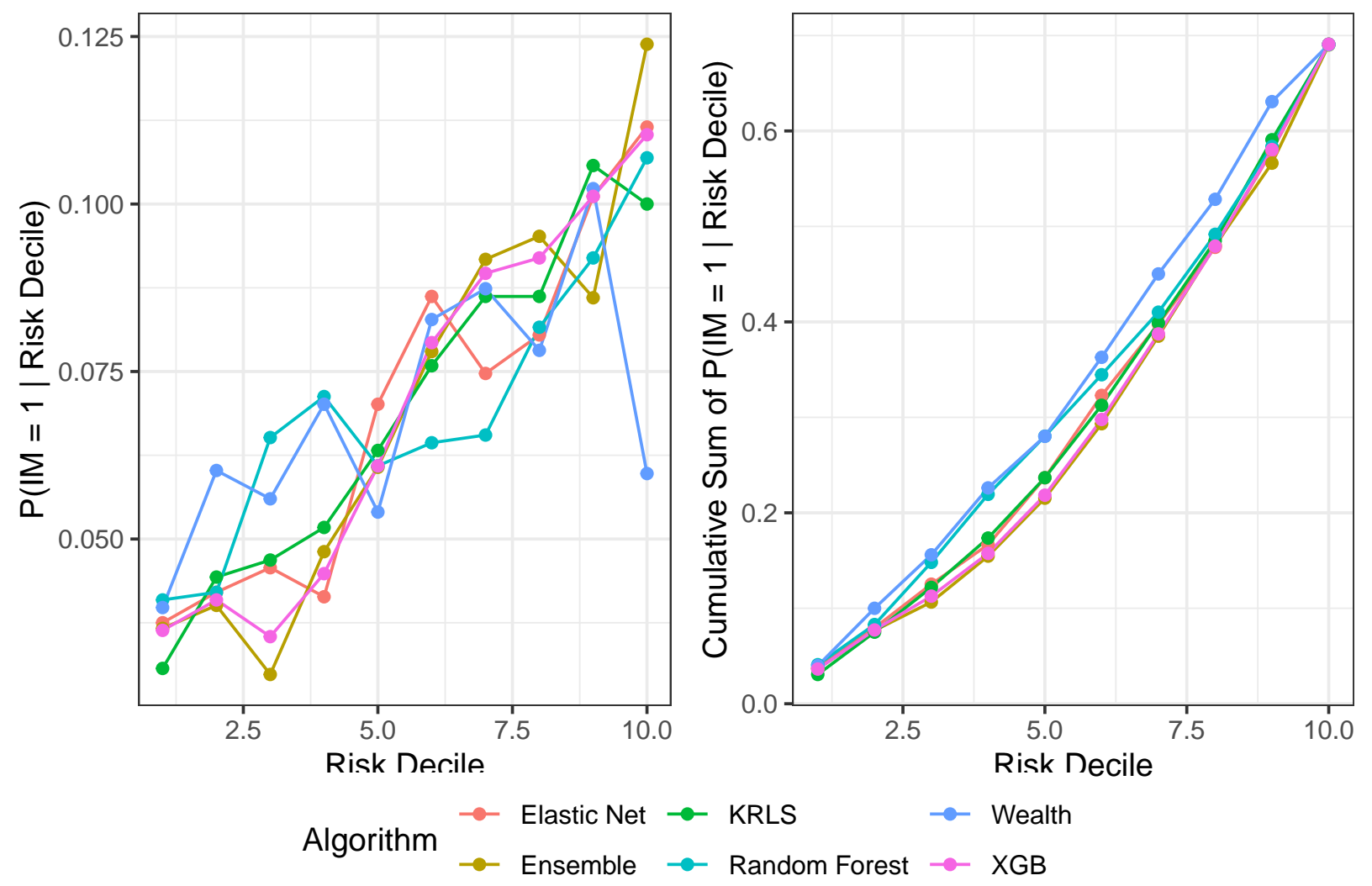

Figure 7: Probability of a mortality given that the observation is in a particular risk decile 
medRxiv preprint doi: https://doi.org/10.1101/2021.07.20.21260818; this version posted July 23, 2021. The copyright holder for this preprint (which was not certified by peer review) is the author/funder, who has granted medRxiv a license to display the preprint in perpetuity. All rights reserved. No reuse allowed without permission.
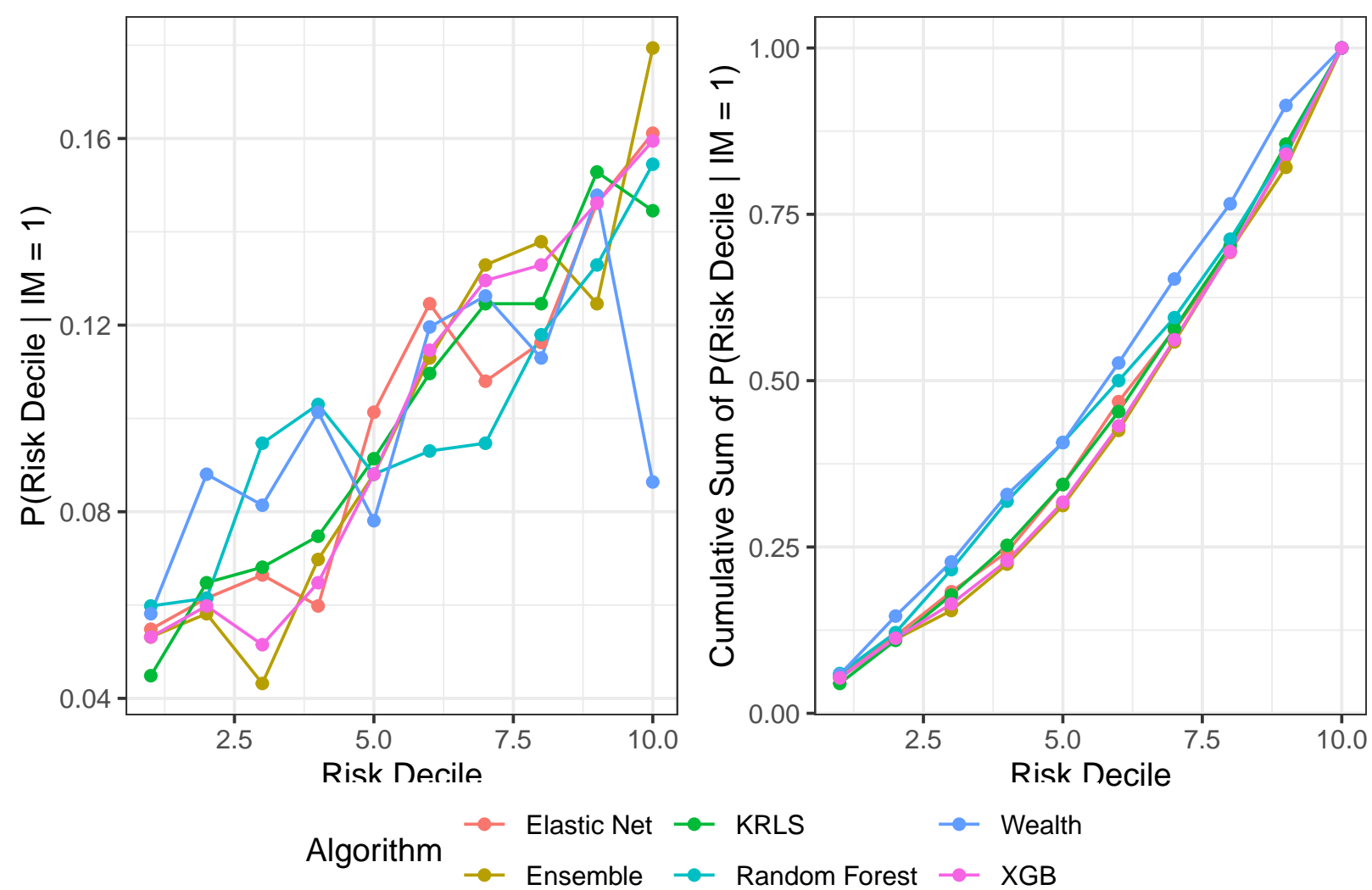

Figure 8: Probability of membership in a particular risk decile given a mortality 


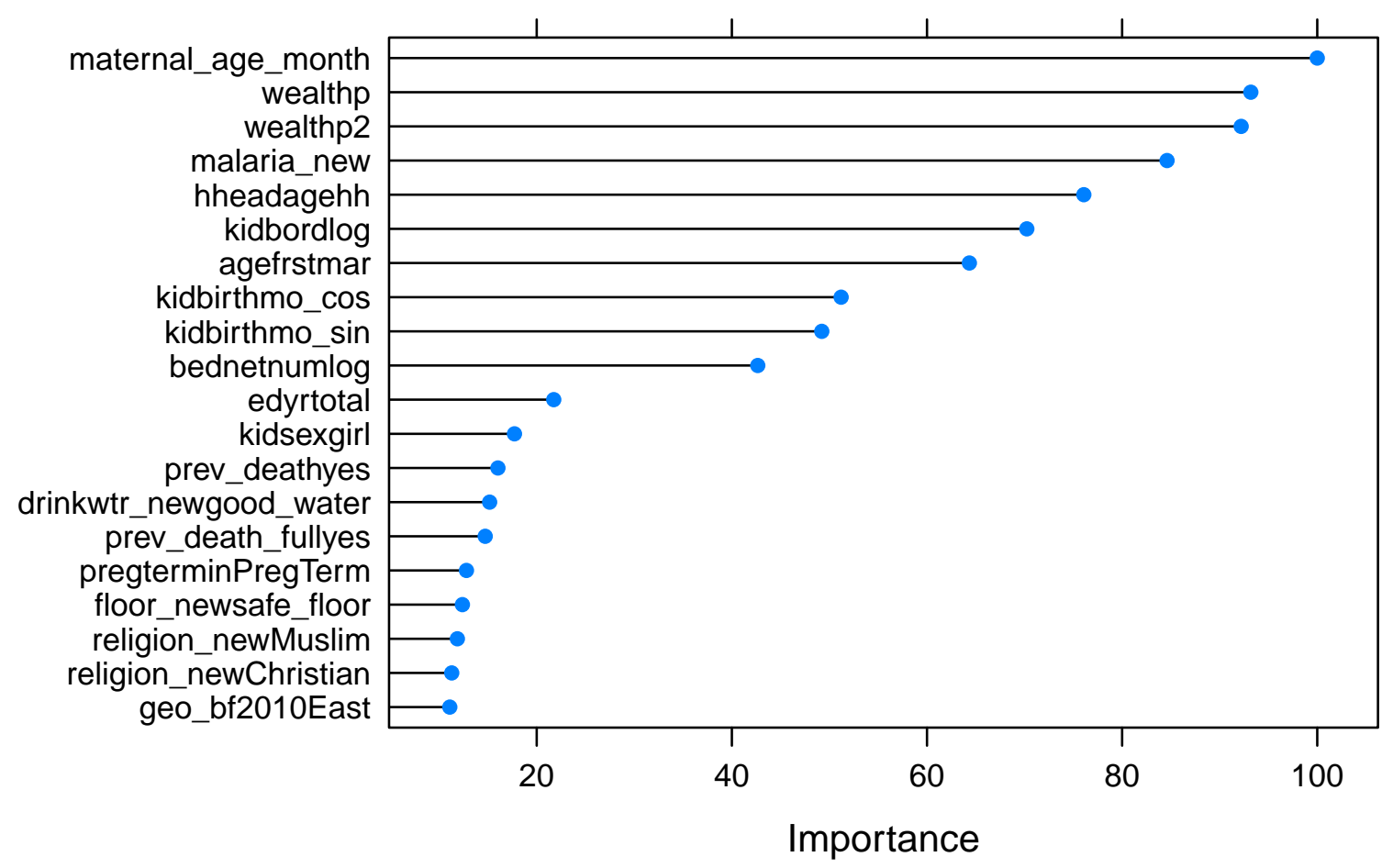

Figure 9: Variable Importance Plot generated by the Random Forest model

\section{B.4.6 Variable Importance Plot}

In this section we will show some measures to give some indication of the important variables necessary for predicting infant mortality. 


\section{B.4.7 Elastic Net Coefficients}

Table 32: Standardized coefficients from the optimal elastic net model after 10-fold crossvalidation.

\begin{tabular}{|c|c|c|}
\hline & Coefficients & Standardized Coefficients \\
\hline (Intercept) & -2.4629 & -2.4629 \\
\hline pregterminPregTerm & 0.0037 & 0.0012 \\
\hline agefrstmar & - & - \\
\hline hheadagehh & - & - \\
\hline urbanRural & 0.0872 & 0.036 \\
\hline kidsexgirl & - & - \\
\hline hheadsexfemale & - & - \\
\hline edyrtotal & -0.0146 & -0.0363 \\
\hline drinkwtr_newgood_water & -0.147 & -0.0632 \\
\hline floor_newsafe_floor & - & - \\
\hline maternal_age_month & $-7 \mathrm{e}-04$ & -0.0601 \\
\hline cookfuel_newclean_fuel & -0.1543 & -0.0203 \\
\hline toilettype_newpit & - & - \\
\hline toilettype_newunimproved & - & - \\
\hline religion_newChristian & - & - \\
\hline religion_newHindu & - & - \\
\hline religion_newMuslim & - & - \\
\hline religion_newOther & 0.2417 & 0.0734 \\
\hline bednetnumlog & - & - \\
\hline prev_deathyes & 0.3052 & 0.1283 \\
\hline prev_death_fullyes & 0.1653 & 0.0781 \\
\hline malaria_new & - & - \\
\hline kidbordlog & - & - \\
\hline kidbirthmo_sin & 0.0234 & 0.0168 \\
\hline kidbirthmo_cos & $-4 \mathrm{e}-04$ & $-3 e-04$ \\
\hline geo_bf2010Cascades & - & - \\
\hline geo_bf2010Center & - & - \\
\hline geo_bf2010Center-East & -0.1013 & -0.0276 \\
\hline geo_bf2010Center-North & -0.1151 & -0.0304 \\
\hline geo_bf2010Center-West & - & - \\
\hline geo_bf2010Center-South & - & - \\
\hline geo_bf2010East & 0.1602 & 0.0477 \\
\hline geo_bf2010Hauts Basins & -0.0208 & -0.0058 \\
\hline geo_bf2010North & - & - \\
\hline geo_bf2010Central Plateau & -0.057 & -0.0137 \\
\hline geo_bf2010Sahel & 0.1855 & 0.0531 \\
\hline geo_bf2010South-West & 0.2369 & 0.0569 \\
\hline wealthp & - & - \\
\hline wealthp2 & -0.1237 & -0.0369 \\
\hline
\end{tabular}


medRxiv preprint doi: https://doi.org/10.1101/2021.07.20.21260818; this version posted July 23, 2021. The copyright holder for this preprint (which was not certified by peer review) is the author/funder, who has granted medRxiv a license to display the preprint in perpetuity.

All rights reserved. No reuse allowed without permission.

\section{B.5 Cameroon}

This sample was taken in the year 2011. There are 8450 observations in the dataset, and 6761 observations were used for the training set. Also, 591 observations were removed due to missing values. Finally, there were 564 deaths for the full dataset, and 452 deaths in the training set. 
medRxiv preprint doi: https://doi.org/10.1101/2021.07.20.21260818; this version posted July 23, 2021. The copyright holder for this preprint (which was not certified by peer review) is the author/funder, who has granted medRxiv a license to display the preprint in perpetuity.

All rights reserved. No reuse allowed without permission. 


\section{B.5.1 Variables Used}

Table 33: Variables included in the model for Cameroon

\begin{tabular}{|c|c|}
\hline Variable & Туре \\
\hline mortality.under $12 \mathrm{~m}$ & categorical \\
\hline pregtermin & categorical \\
\hline agefrstmar & numeric \\
\hline hheadagehh & numeric \\
\hline urban & categorical \\
\hline kidsex & categorical \\
\hline hheadsex & categorical \\
\hline edyrtotal & numeric \\
\hline drinkwtr_new & categorical \\
\hline floor_new & categorical \\
\hline maternal_age_month & numeric \\
\hline cookfuel_new & categorical \\
\hline toilettype_new & categorical \\
\hline religion_new & categorical \\
\hline bednetnumlog & numeric \\
\hline prev_death & categorical \\
\hline prev_death_full & categorical \\
\hline malaria_new & numeric \\
\hline kidbordlog & numeric \\
\hline kidbirthmo_sin & numeric \\
\hline kidbirthmo_cos & numeric \\
\hline geo_cm2011 & categorical \\
\hline wealthp & numeric \\
\hline wealthp2 & numeric \\
\hline
\end{tabular}




\section{B.5.2 Mortality Breakdown by Wealth Quintile}

\section{B.5.3 Optimal Parameters}

These are the algorithm parameters selected after cross-validation:

Table 34: Optimal Parameters for the Elastic Net algorithm

\begin{tabular}{|c|c|}
\hline alpha & lambda \\
\hline 0.1 & 0.0163135 \\
\hline
\end{tabular}

Table 35: Optimal Parameters for the Random Forest algorithm

\begin{tabular}{|c|}
\hline mtry \\
\hline 10 \\
\hline
\end{tabular}

Table 36: Optimal Parameters for the XGB algorithm

\begin{tabular}{|c|c|c|c|c|c|c|}
\hline nrounds & max_depth & eta & gamma & colsample_bytree & min_child_weight & subsample \\
\hline 50 & 1 & 0.4 & 0 & 0.8 & 1 & 0.75 \\
\hline
\end{tabular}

Table 37: Optimal Parameters for the KRLS algorithm

\begin{tabular}{|c|c|c|c|}
\hline loss & epsilon & $\mathbf{b}$ & lambda \\
\hline logistic & 0.005 & 72 & 0.0004365 \\
\hline
\end{tabular}


medRxiv preprint doi: https://doi.org/10.1101/2021.07.20.21260818; this version posted July 23, 2021. The copyright holder for this preprint (which was not certified by peer review) is the author/funder, who has granted medRxiv a license to display the preprint in perpetuity.

\section{B.5.4 Table of Results}

Table 38: Manual Cross-Validation Results

\begin{tabular}{|c|c|c|c|c|c|c|c|c|c|}
\hline Algorithm & Recall 10 & Recall 20 & ROC & AUC & MRD & MRR & MSE & F1 & Efficiency Gain \\
\hline Ensemble & 0.239 & 0.365 & 0.620 & 0.182 & 0.021 & 1.313 & 0.061 & 0.926 & 2.040 \\
\hline KRLS & 0.146 & 0.266 & 0.584 & 0.092 & 0.005 & 1.074 & 0.062 & 0.920 & 1.247 \\
\hline Elastic Net & 0.153 & 0.272 & 0.584 & 0.095 & 0.006 & 1.085 & 0.062 & 0.921 & 1.304 \\
\hline Random Forest & 0.217 & 0.327 & 0.598 & 0.179 & 0.062 & 1.827 & 0.061 & 0.925 & 1.850 \\
\hline Wealth & 0.117 & 0.252 & 0.547 & 0.084 & 0.137 & 2.072 & 0.910 & 0.918 & 1.000 \\
\hline XGB & 0.142 & 0.274 & 0.595 & 0.114 & 0.013 & 1.199 & 0.062 & 0.920 & 1.210 \\
\hline
\end{tabular}

Table 39: Distribution of individuals in the top risk decile of each algorithm among each wealth decile

\begin{tabular}{l|r|r|r|r|c}
\hline Wealth Decile & Elastic Net & Ensemble & KRLS & Random Forest & XGB \\
\hline 1 & 0.272 & 0.218 & 0.246 & 0.188 & 0.204 \\
\hline 2 & 0.212 & 0.186 & 0.223 & 0.145 & 0.237 \\
\hline 3 & 0.128 & 0.109 & 0.131 & 0.104 & 0.116 \\
\hline 4 & 0.106 & 0.078 & 0.116 & 0.064 & 0.091 \\
\hline 5 & 0.085 & 0.107 & 0.094 & 0.094 & 0.097 \\
\hline 6 & 0.072 & 0.085 & 0.073 & 0.091 & 0.082 \\
\hline 7 & 0.050 & 0.074 & 0.055 & 0.075 & 0.047 \\
\hline 8 & 0.022 & 0.039 & 0.024 & 0.046 & 0.033 \\
\hline 9 & 0.036 & 0.052 & 0.021 & 0.079 & 0.030 \\
\hline 10 & 0.015 & 0.058 & 0.013 & 0.110 & 0.060 \\
\hline
\end{tabular}

\section{B.5.5 Performance Plots}

Below are various plots showing the performance for each model considered in Cameroon. 
medRxiv preprint doi: https://doi.org/10.1101/2021.07.20.21260818; this version posted July 23, 2021. The copyright holder for this preprint (which was not certified by peer review) is the author/funder, who has granted medRxiv a license to display the preprint in perpetuity. All rights reserved. No reuse allowed without permission.

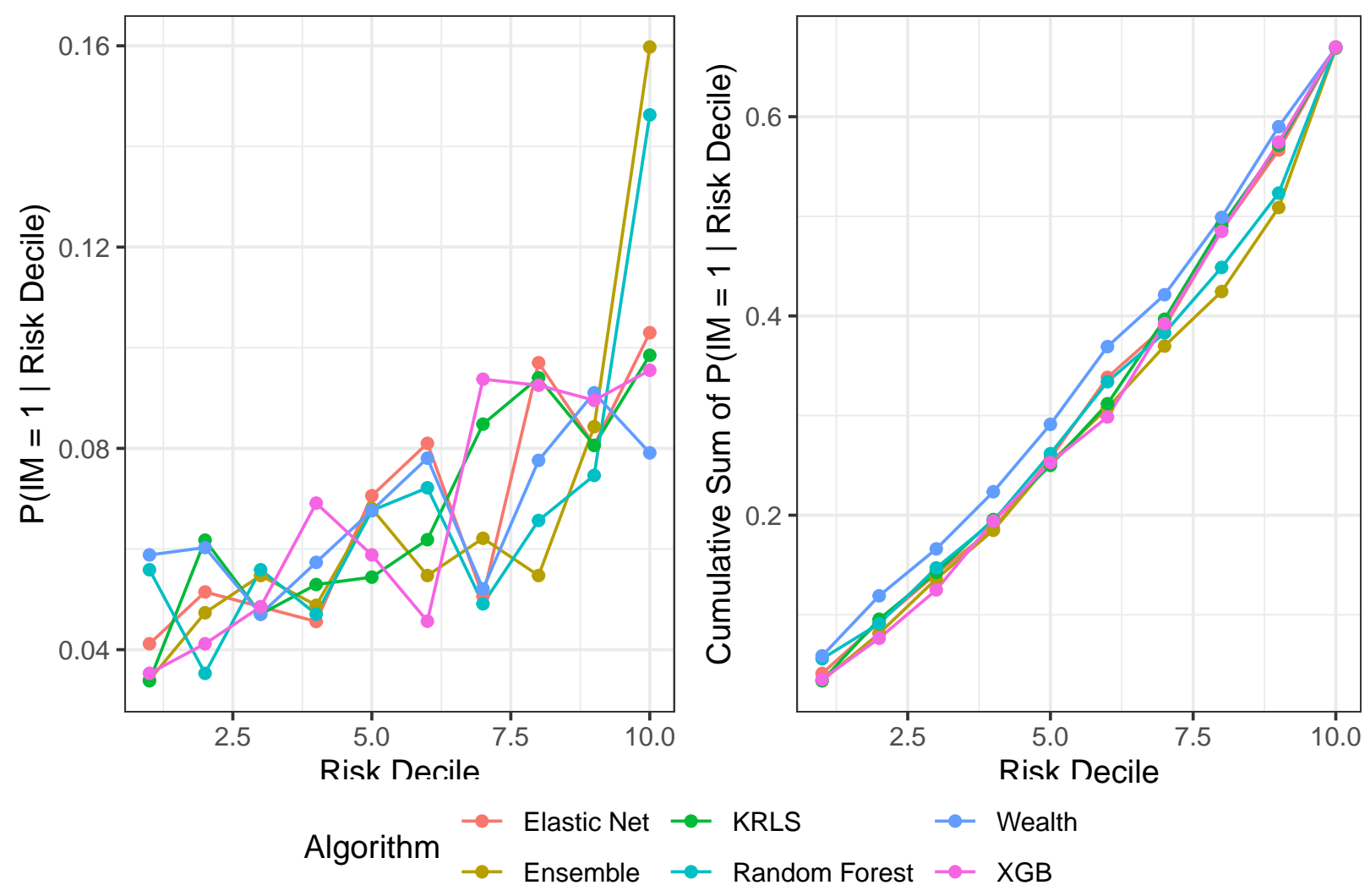

Figure 10: Probability of a mortality given that the observation is in a particular risk decile 
medRxiv preprint doi: https://doi.org/10.1101/2021.07.20.21260818; this version posted July 23, 2021. The copyright holder for this preprint (which was not certified by peer review) is the author/funder, who has granted medRxiv a license to display the preprint in perpetuity. All rights reserved. No reuse allowed without permission.
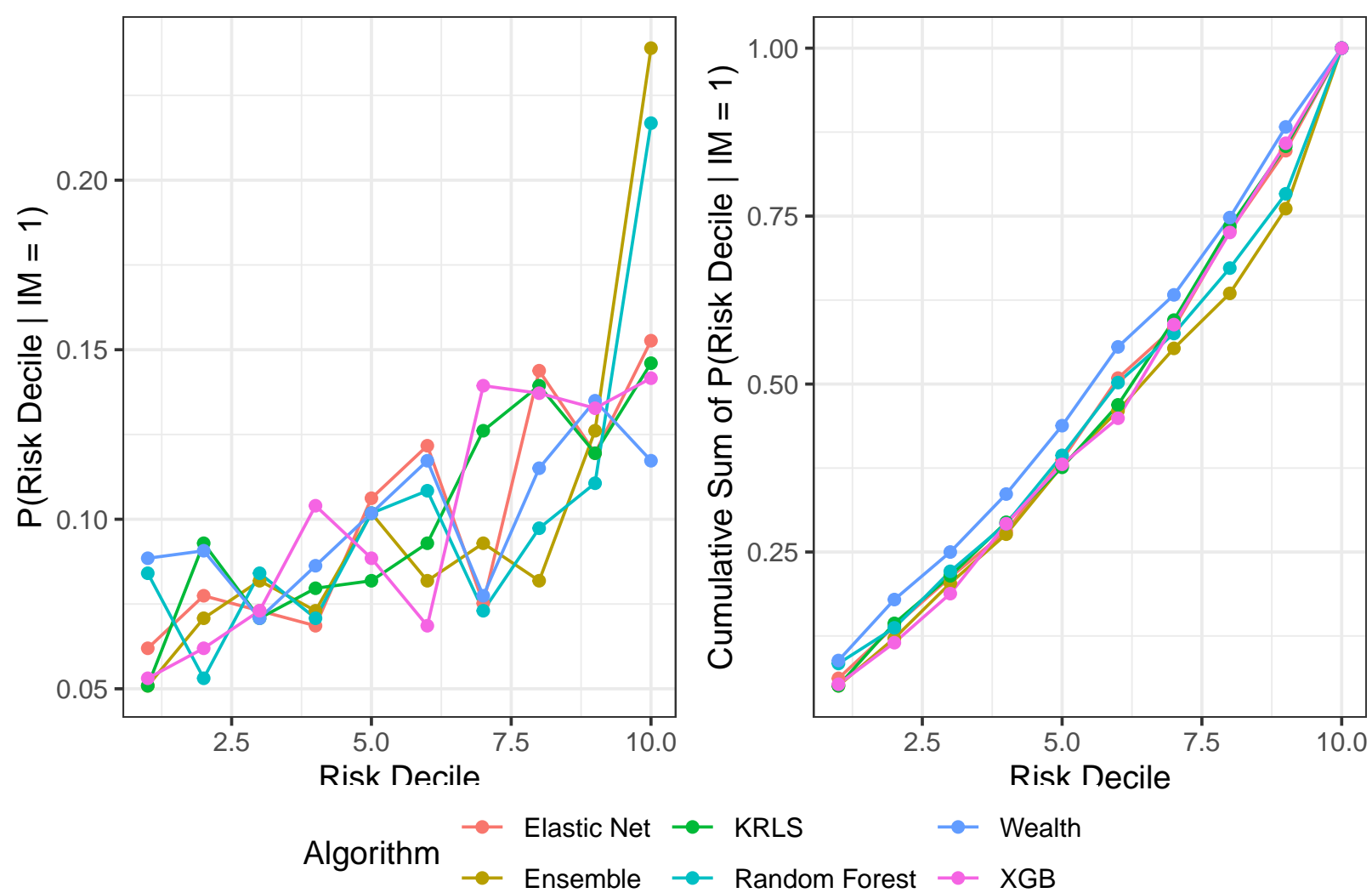

Figure 11: Probability of membership in a particular risk decile given a mortality 


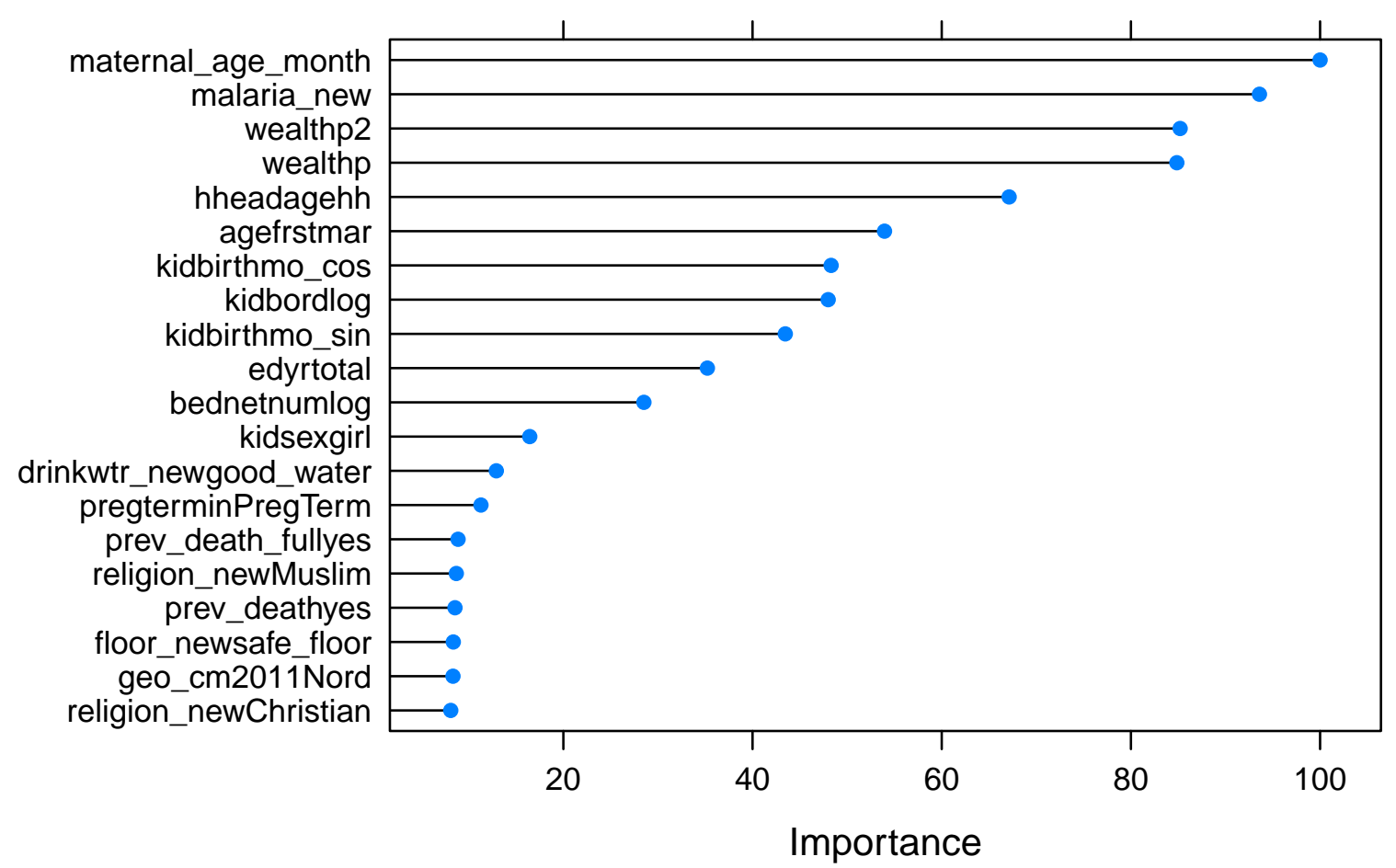

Figure 12: Variable Importance Plot generated by the Random Forest model

\section{B.5.6 Variable Importance Plot}

In this section we will show some measures to give some indication of the important variables necessary for predicting infant mortality. 
medRxiv preprint doi: https://doi.org/10.1101/2021.07.20.21260818; this version posted July 23, 2021. The copyright holder for this preprint (which was not certified by peer review) is the author/funder, who has granted medRxiv a license to display the preprint in perpetuity.

All rights reserved. No reuse allowed without permission.

\section{B.5.7 Elastic Net Coefficients}

Table 40: Standardized coefficients from the optimal elastic net model after 10-fold crossvalidation.

\begin{tabular}{|c|c|c|}
\hline & Coefficients & Standardized Coefficients \\
\hline (Intercept) & -2.3741 & -2.3741 \\
\hline pregterminPregTerm & 0.1506 & 0.0657 \\
\hline agefrstmar & -0.0041 & -0.0162 \\
\hline hheadagehh & 0.0036 & 0.0483 \\
\hline urbanRural & - & - \\
\hline kidsexgirl & -0.0766 & -0.0383 \\
\hline hheadsexfemale & -0.0127 & -0.0046 \\
\hline edyrtotal & -0.0165 & -0.0654 \\
\hline drinkwtr_newgood_water & - & - \\
\hline floor_newsafe_floor & -0.0599 & -0.0299 \\
\hline maternal_age_month & $-9 \mathrm{e}-04$ & -0.0683 \\
\hline cookfuel_newclean_fuel & - & - \\
\hline toilettype_newpit & - & - \\
\hline toilettype_newunimproved & $2 \mathrm{e}-04$ & $1 \mathrm{e}-04$ \\
\hline religion_newChristian & - & - \\
\hline religion_newHindu & - & - \\
\hline religion_newMuslim & 0.0559 & 0.0242 \\
\hline religion_newOther & -0.0625 & -0.0157 \\
\hline bednetnumlog & 0.1042 & 0.053 \\
\hline prev_deathyes & 0.3372 & 0.1274 \\
\hline prev_death_fullyes & - & - \\
\hline malaria_new & -0.3486 & -0.037 \\
\hline kidbordlog & 0.0233 & 0.0162 \\
\hline kidbirthmo_sin & $9 \mathrm{e}-04$ & $6 \mathrm{e}-04$ \\
\hline kidbirthmo_cos & -0.0835 & -0.0582 \\
\hline geo_cm2011Centre & - & - \\
\hline geo_cm2011Douala & -0.2219 & -0.0542 \\
\hline geo_cm2011Est & -0.2023 & -0.0519 \\
\hline geo_cm2011Extrême-Nord & 0.1703 & 0.0628 \\
\hline geo_cm2011Littoral & - & - \\
\hline geo_cm2011Nord & 0.1748 & 0.0606 \\
\hline geo_cm2011Nord-Ouest & -0.0083 & -0.0022 \\
\hline geo_cm2011Ouest & -0.0285 & -0.0083 \\
\hline geo_cm2011Sud & - & - \\
\hline geo_cm2011Sud-Ouest & 0.1559 & 0.0355 \\
\hline geo_cm2011Yaoundé & - & - \\
\hline wealthp & - & - \\
\hline wealthp2 & - & - \\
\hline
\end{tabular}


medRxiv preprint doi: https://doi.org/10.1101/2021.07.20.21260818; this version posted July 23, 2021. The copyright holder for this preprint (which was not certified by peer review) is the author/funder, who has granted medRxiv a license to display the preprint in perpetuity.

All rights reserved. No reuse allowed without permission.

\section{B.6 Cote d'Ivoire}

This sample was taken in the year 2011. There are 5211 observations in the dataset, and 4169 observations were used for the training set. Also, 734 observations were removed due to missing values. Finally, there were 421 deaths for the full dataset, and 337 deaths in the training set. 
medRxiv preprint doi: https://doi.org/10.1101/2021.07.20.21260818; this version posted July 23, 2021. The copyright holder for this preprint (which was not certified by peer review) is the author/funder, who has granted medRxiv a license to display the preprint in perpetuity.

All rights reserved. No reuse allowed without permission. 


\section{B.6.1 Variables Used}

Table 41: Variables included in the model for Cote d'Ivoire

\begin{tabular}{|l|l|}
\hline Variable & Type \\
\hline mortality.under12m & categorical \\
\hline pregtermin & categorical \\
\hline agefrstmar & numeric \\
\hline hheadagehh & numeric \\
\hline urban & categorical \\
\hline kidsex & categorical \\
\hline hheadsex & categorical \\
\hline edyrtotal & numeric \\
\hline drinkwtr_new & categorical \\
\hline floor_new & categorical \\
\hline maternal_age_month & numeric \\
\hline cookfuel_new & categorical \\
\hline toilettype_new & categorical \\
\hline religion_new & categorical \\
\hline bednetnumlog & numeric \\
\hline prev_death & categorical \\
\hline prev_death_full & categorical \\
\hline malaria_new & numeric \\
\hline kidbordlog & numeric \\
\hline kidbirthmo_sin & numeric \\
\hline kidbirthmo_cos & \\
\hline wealthp & numealthp2 \\
\hline numic
\end{tabular}




\section{B.6.2 Mortality Breakdown by Wealth Quintile}

\section{B.6.3 Optimal Parameters}

These are the algorithm parameters selected after cross-validation:

Table 42: Optimal Parameters for the Elastic Net algorithm

\begin{tabular}{|c|c|}
\hline alpha & lambda \\
\hline 1 & 0.0029452 \\
\hline
\end{tabular}

Table 43: Optimal Parameters for the Random Forest algorithm

\begin{tabular}{|c|}
\hline mtry \\
\hline 2 \\
\hline
\end{tabular}

Table 44: Optimal Parameters for the XGB algorithm

\begin{tabular}{|c|c|c|c|c|c|c|}
\hline nrounds & max_depth & eta & gamma & colsample_bytree & min_child_weight & subsample \\
\hline 50 & 1 & 0.3 & 0 & 0.6 & 1 & 1 \\
\hline
\end{tabular}

Table 45: Optimal Parameters for the KRLS algorithm

\begin{tabular}{|c|c|c|c|}
\hline loss & epsilon & $\mathbf{b}$ & lambda \\
\hline logistic & 0.005 & 50 & 0.0002803 \\
\hline
\end{tabular}


medRxiv preprint doi: https://doi.org/10.1101/2021.07.20.21260818; this version posted July 23, 2021. The copyright holder for this preprint (which was not certified by peer review) is the author/funder, who has granted medRxiv a license to display the preprint in perpetuity.

\section{B.6.4 Table of Results}

Table 46: Manual Cross-Validation Results

\begin{tabular}{|c|c|c|c|c|c|c|c|c|c|}
\hline Algorithm & Recall 10 & Recall 20 & ROC & AUC & MRD & MRR & MSE & F1 & Efficiency Gain \\
\hline Ensemble & 0.208 & 0.395 & 0.654 & 0.149 & 0.018 & 1.241 & 0.073 & 0.919 & 1.943 \\
\hline KRLS & 0.208 & 0.330 & 0.638 & 0.146 & 0.016 & 1.205 & 0.073 & 0.920 & 1.947 \\
\hline Elastic Net & 0.205 & 0.321 & 0.629 & 0.132 & 0.016 & 1.205 & 0.073 & 0.920 & 1.918 \\
\hline Random Forest & 0.217 & 0.353 & 0.622 & 0.154 & 0.024 & 1.432 & 0.073 & 0.921 & 2.027 \\
\hline Wealth & 0.107 & 0.199 & 0.529 & 0.098 & 0.076 & 2.132 & 0.809 & 0.911 & 1.000 \\
\hline XGB & 0.193 & 0.324 & 0.619 & 0.136 & 0.015 & 1.185 & 0.073 & 0.919 & 1.806 \\
\hline
\end{tabular}

Table 47: Distribution of individuals in the top risk decile of each algorithm among each wealth decile

\begin{tabular}{l|r|r|r|r|c}
\hline Wealth Decile & Elastic Net & Ensemble & KRLS & Random Forest & XGB \\
\hline 1 & 0.088 & 0.124 & 0.105 & 0.185 & 0.154 \\
\hline 2 & 0.089 & 0.118 & 0.113 & 0.127 & 0.084 \\
\hline 3 & 0.115 & 0.124 & 0.139 & 0.098 & 0.112 \\
\hline 4 & 0.124 & 0.117 & 0.129 & 0.088 & 0.102 \\
\hline 5 & 0.117 & 0.102 & 0.132 & 0.066 & 0.105 \\
\hline 6 & 0.139 & 0.117 & 0.112 & 0.083 & 0.110 \\
\hline 7 & 0.125 & 0.115 & 0.120 & 0.065 & 0.123 \\
\hline 8 & 0.105 & 0.100 & 0.090 & 0.117 & 0.098 \\
\hline 9 & 0.049 & 0.054 & 0.032 & 0.059 & 0.061 \\
\hline 10 & 0.046 & 0.039 & 0.024 & 0.110 & 0.049 \\
\hline
\end{tabular}

\section{B.6.5 Performance Plots}

Below are various plots showing the performance for each model considered in Cote d'Ivoire. 
medRxiv preprint doi: https://doi.org/10.1101/2021.07.20.21260818; this version posted July 23, 2021. The copyright holder for this preprint (which was not certified by peer review) is the author/funder, who has granted medRxiv a license to display the preprint in perpetuity. All rights reserved. No reuse allowed without permission.

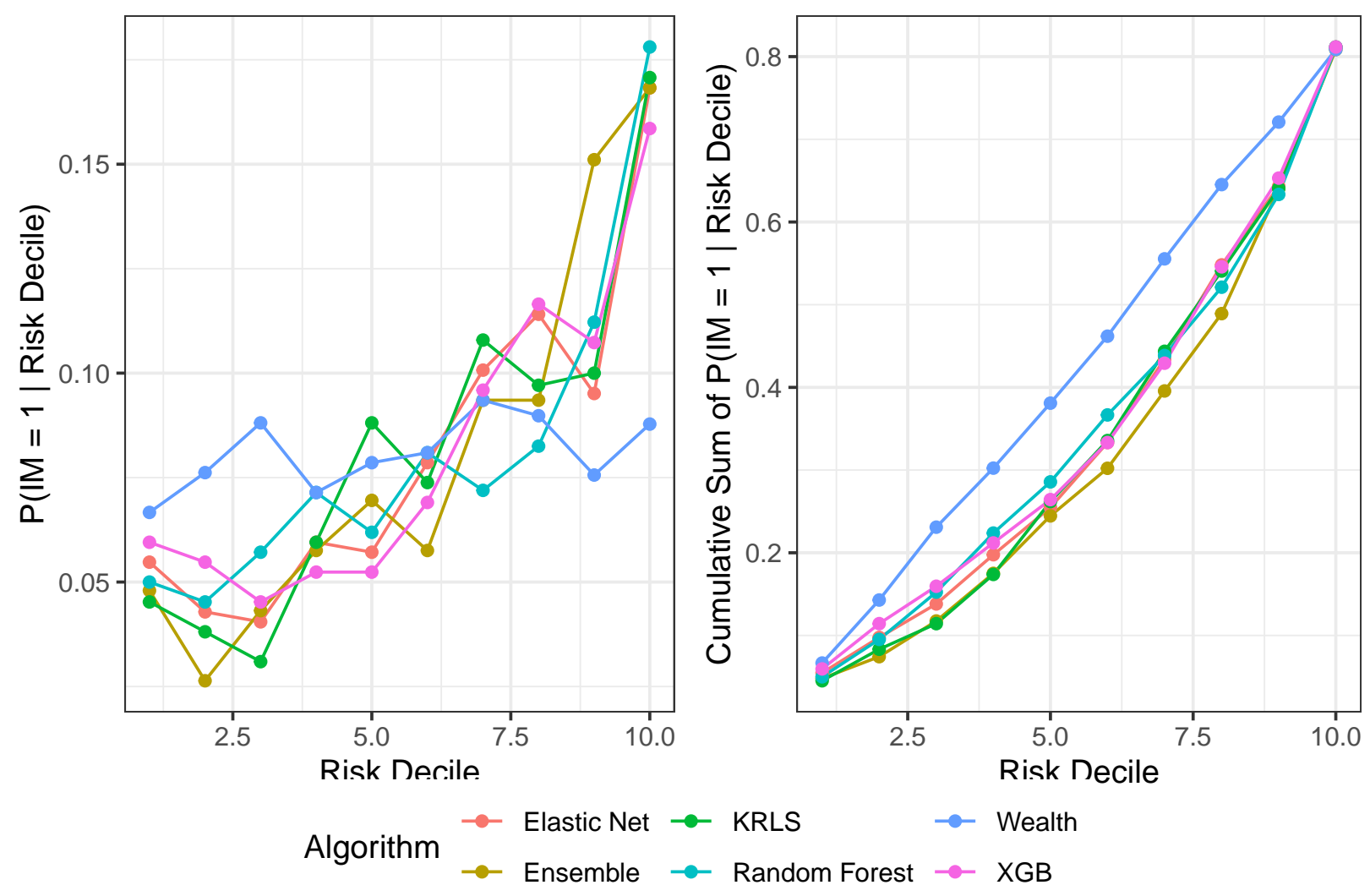

Figure 13: Probability of a mortality given that the observation is in a particular risk decile 
medRxiv preprint doi: https://doi.org/10.1101/2021.07.20.21260818; this version posted July 23, 2021. The copyright holder for this preprint (which was not certified by peer review) is the author/funder, who has granted medRxiv a license to display the preprint in perpetuity. All rights reserved. No reuse allowed without permission.

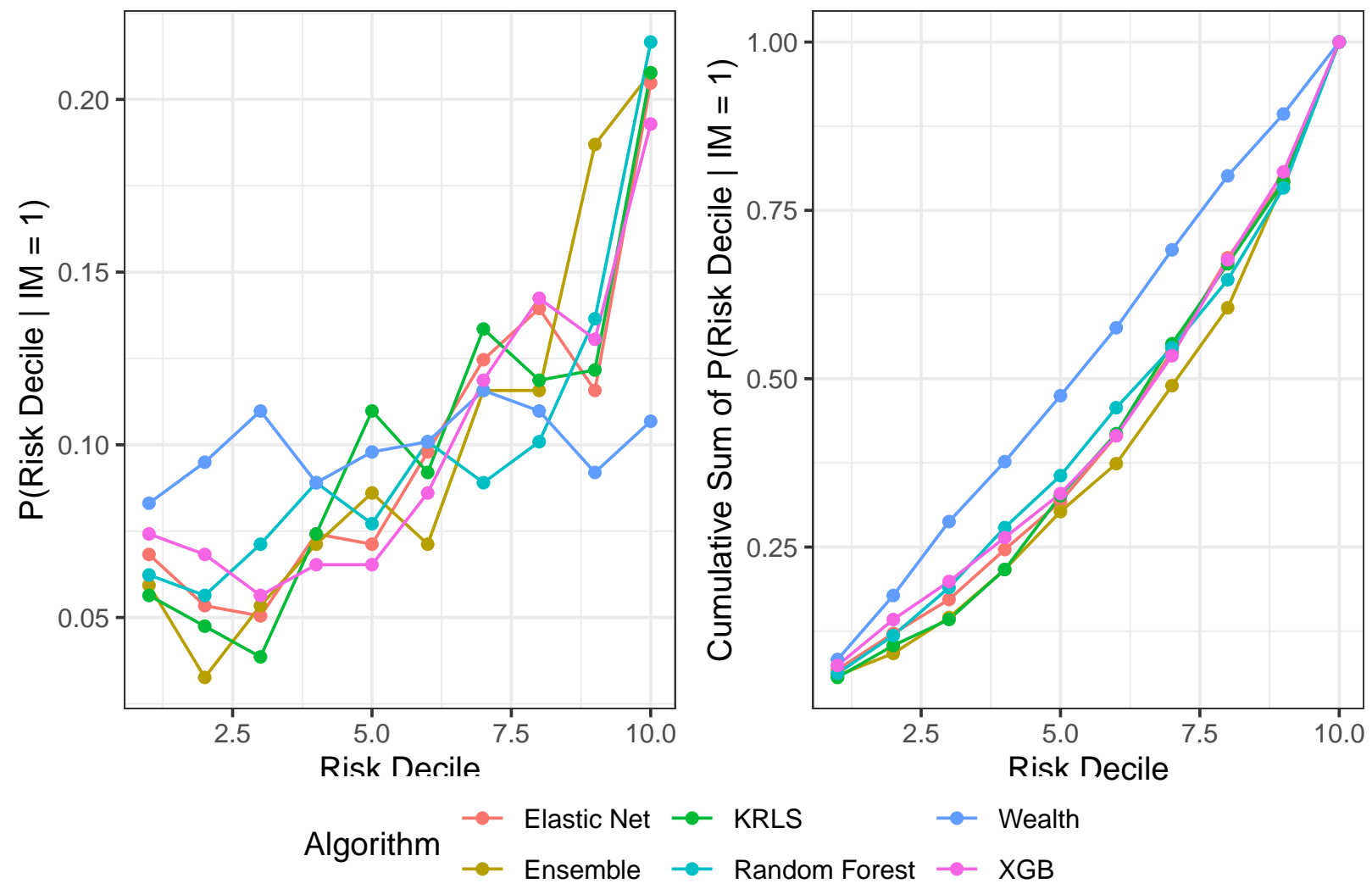

Figure 14: Probability of membership in a particular risk decile given a mortality 


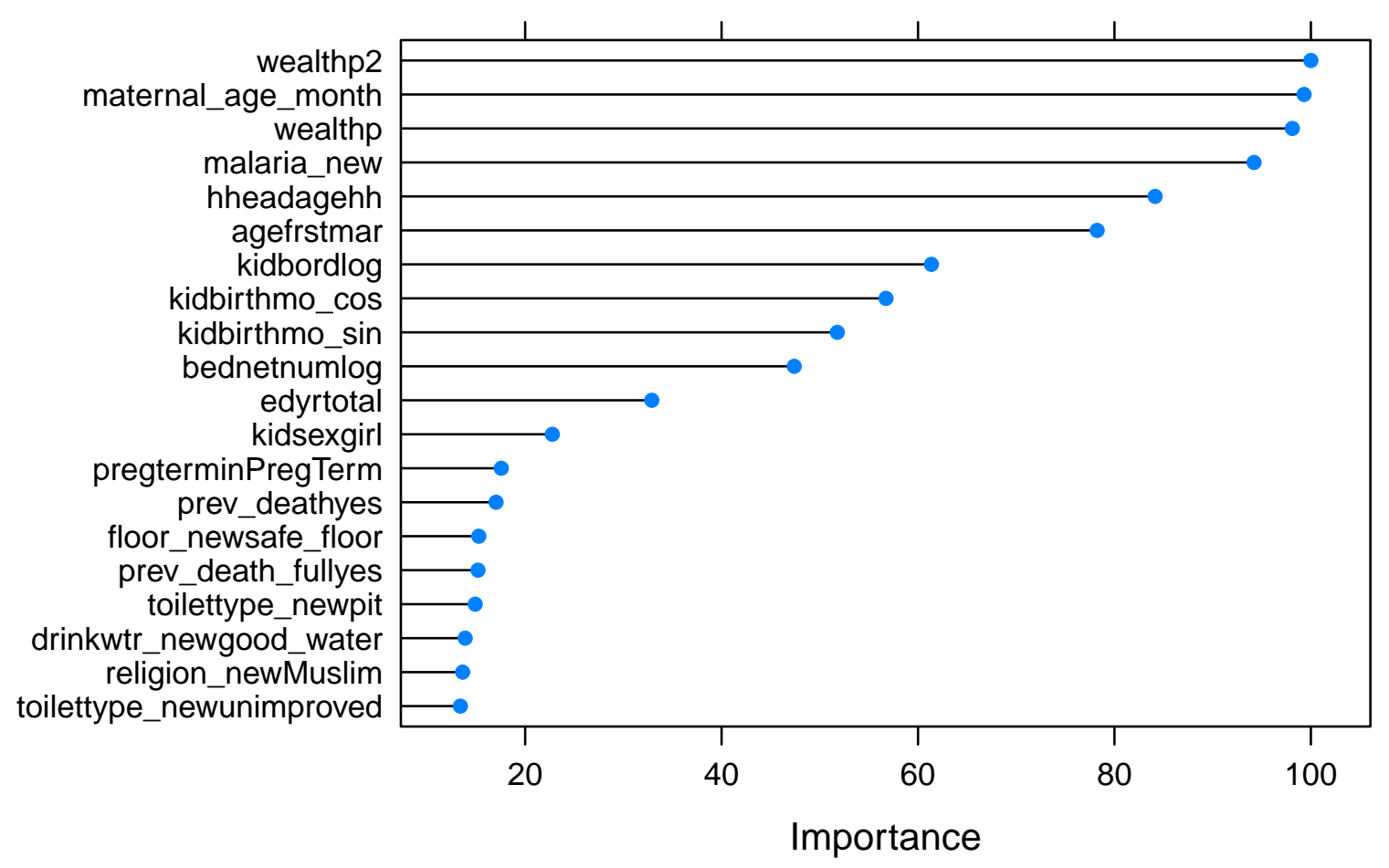

Figure 15: Variable Importance Plot generated by the Random Forest model

\section{B.6.6 Variable Importance Plot}

In this section we will show some measures to give some indication of the important variables necessary for predicting infant mortality. 
medRxiv preprint doi: https://doi.org/10.1101/2021.07.20.21260818; this version posted July 23, 2021. The copyright holder for this preprint (which was not certified by peer review) is the author/funder, who has granted medRxiv a license to display the preprint in perpetuity.

All rights reserved. No reuse allowed without permission.

\section{B.6.7 Elastic Net Coefficients}

Table 48: Standardized coefficients from the optimal elastic net model after 10-fold crossvalidation.

\begin{tabular}{|c|c|c|}
\hline & Coefficients & Standardized Coefficients \\
\hline (Intercept) & -1.3584 & -1.3584 \\
\hline pregterminPregTerm & 0.1588 & 0.0614 \\
\hline agefrstmar & - & - \\
\hline hheadagehh & - & - \\
\hline urbanRural & 0.3648 & 0.1701 \\
\hline kidsexgirl & -0.5679 & -0.284 \\
\hline hheadsexfemale & - & - \\
\hline edyrtotal & - & - \\
\hline drinkwtr_newgood_water & 0.0773 & 0.0334 \\
\hline floor_newsafe_floor & - & - \\
\hline maternal_age_month & $-8 \mathrm{e}-04$ & -0.065 \\
\hline cookfuel_newclean_fuel & 0.0267 & 0.0068 \\
\hline toilettype_newpit & 0.0909 & 0.0452 \\
\hline toilettype_newunimproved & - & - \\
\hline religion_newChristian & -0.0845 & -0.04 \\
\hline religion_newHindu & - & - \\
\hline religion_newMuslim & 0.0192 & 0.0096 \\
\hline religion_newOther & - & - \\
\hline bednetnumlog & - & - \\
\hline prev_deathyes & 0.5558 & 0.2327 \\
\hline prev_death_fullyes & 0.0971 & 0.0444 \\
\hline malaria_new & -2.1569 & -0.1071 \\
\hline kidbordlog & - & - \\
\hline kidbirthmo_sin & 0.0599 & 0.0422 \\
\hline kidbirthmo_cos & - & - \\
\hline wealthp & - & - \\
\hline wealthp2 & - & - \\
\hline
\end{tabular}


medRxiv preprint doi: https://doi.org/10.1101/2021.07.20.21260818; this version posted July 23, 2021. The copyright holder for this preprint (which was not certified by peer review) is the author/funder, who has granted medRxiv a license to display the preprint in perpetuity.

All rights reserved. No reuse allowed without permission.

\section{B.7 Ghana}

This sample was taken in the year 2014. There are 4203 observations in the dataset, and 3364 observations were used for the training set. Also, 345 observations were removed due to missing values. Finally, there were 189 deaths for the full dataset, and 152 deaths in the training set. 
medRxiv preprint doi: https://doi.org/10.1101/2021.07.20.21260818; this version posted July 23, 2021. The copyright holder for this preprint (which was not certified by peer review) is the author/funder, who has granted medRxiv a license to display the preprint in perpetuity.

All rights reserved. No reuse allowed without permission. 


\section{B.7.1 Variables Used}

Table 49: Variables included in the model for Ghana

\begin{tabular}{|l|l|}
\hline Variable & Type \\
\hline mortality.under12m & categorical \\
\hline pregtermin & categorical \\
\hline agefrstmar & numeric \\
\hline hheadagehh & numeric \\
\hline urban & categorical \\
\hline kidsex & categorical \\
\hline hheadsex & categorical \\
\hline edyrtotal & numeric \\
\hline drinkwtr_new & categorical \\
\hline floor_new & categorical \\
\hline maternal_age_month & numeric \\
\hline cookfuel_new & categorical \\
\hline toilettype_new & categorical \\
\hline religion_new & categorical \\
\hline bednetnumlog & numeric \\
\hline prev_death & categorical \\
\hline prev_death_full & categorical \\
\hline malaria_new & numeric \\
\hline kidbordlog & numeric \\
\hline kidbirthmo_sin & numeric \\
\hline kidbirthmo_cos & \\
\hline wealthp & numealthp2 \\
\hline numeric
\end{tabular}




\section{B.7.2 Mortality Breakdown by Wealth Quintile}

\section{B.7.3 Optimal Parameters}

These are the algorithm parameters selected after cross-validation:

Table 50: Optimal Parameters for the Elastic Net algorithm

\begin{tabular}{|c|c|}
\hline alpha & lambda \\
\hline 0.6785714 & 0.0090749 \\
\hline
\end{tabular}

Table 51: Optimal Parameters for the Random Forest algorithm

\begin{tabular}{|c|}
\hline mtry \\
\hline 2 \\
\hline
\end{tabular}

Table 52: Optimal Parameters for the XGB algorithm

\begin{tabular}{|c|c|c|c|c|c|c|}
\hline nrounds & max_depth & eta & gamma & colsample_bytree & min_child_weight & subsample \\
\hline 50 & 1 & 0.3 & 0 & 0.6 & 1 & 1 \\
\hline
\end{tabular}

Table 53: Optimal Parameters for the KRLS algorithm

\begin{tabular}{|c|c|c|c|}
\hline loss & epsilon & $\mathbf{b}$ & lambda \\
\hline logistic & 0.005 & 50 & 0.0086021 \\
\hline
\end{tabular}


medRxiv preprint doi: https://doi.org/10.1101/2021.07.20.21260818; this version posted July 23, 2021. The copyright holder for this preprint (which was not certified by peer review) is the author/funder, who has granted medRxiv a license to display the preprint in perpetuity.

\section{B.7.4 Table of Results}

Table 54: Manual Cross-Validation Results

\begin{tabular}{|c|c|c|c|c|c|c|c|c|c|}
\hline Algorithm & Recall 10 & Recall 20 & ROC & AUC & MRD & MRR & MSE & F1 & Efficiency Gain \\
\hline Ensemble & 0.185 & 0.271 & 0.539 & 0.120 & 0.007 & 1.161 & 0.043 & 0.931 & 3.127 \\
\hline KRLS & 0.165 & 0.290 & 0.567 & 0.065 & 0.000 & 1.009 & 0.043 & 0.931 & 2.796 \\
\hline Elastic Net & 0.185 & 0.304 & 0.519 & 0.062 & 0.002 & 1.048 & 0.043 & 0.932 & 3.127 \\
\hline Random Forest & 0.132 & 0.243 & 0.556 & 0.102 & 0.019 & 1.530 & 0.043 & 0.929 & 2.232 \\
\hline Wealth & 0.059 & 0.210 & 0.568 & 0.046 & -0.038 & 0.889 & 0.993 & 0.926 & 1.000 \\
\hline XGB & 0.172 & 0.285 & 0.539 & 0.079 & 0.006 & 1.135 & 0.043 & 0.931 & 2.901 \\
\hline
\end{tabular}

Table 55: Distribution of individuals in the top risk decile of each algorithm among each wealth decile

\begin{tabular}{l|r|r|r|r|c}
\hline Wealth Decile & Elastic Net & Ensemble & KRLS & Random Forest & XGB \\
\hline 1 & 0.145 & 0.112 & 0.133 & 0.139 & 0.073 \\
\hline 2 & 0.122 & 0.149 & 0.116 & 0.116 & 0.155 \\
\hline 3 & 0.164 & 0.142 & 0.158 & 0.127 & 0.127 \\
\hline 4 & 0.076 & 0.088 & 0.079 & 0.079 & 0.085 \\
\hline 5 & 0.094 & 0.052 & 0.082 & 0.045 & 0.061 \\
\hline 6 & 0.133 & 0.118 & 0.148 & 0.091 & 0.133 \\
\hline 7 & 0.060 & 0.074 & 0.080 & 0.071 & 0.083 \\
\hline 8 & 0.073 & 0.079 & 0.079 & 0.082 & 0.052 \\
\hline 9 & 0.052 & 0.064 & 0.055 & 0.073 & 0.082 \\
\hline 10 & 0.079 & 0.136 & 0.067 & 0.173 & 0.145 \\
\hline
\end{tabular}

\section{B.7.5 Performance Plots}

Below are various plots showing the performance for each model considered in Ghana. 
medRxiv preprint doi: https://doi.org/10.1101/2021.07.20.21260818; this version posted July 23, 2021. The copyright holder for this preprint (which was not certified by peer review) is the author/funder, who has granted medRxiv a license to display the preprint in perpetuity. All rights reserved. No reuse allowed without permission.

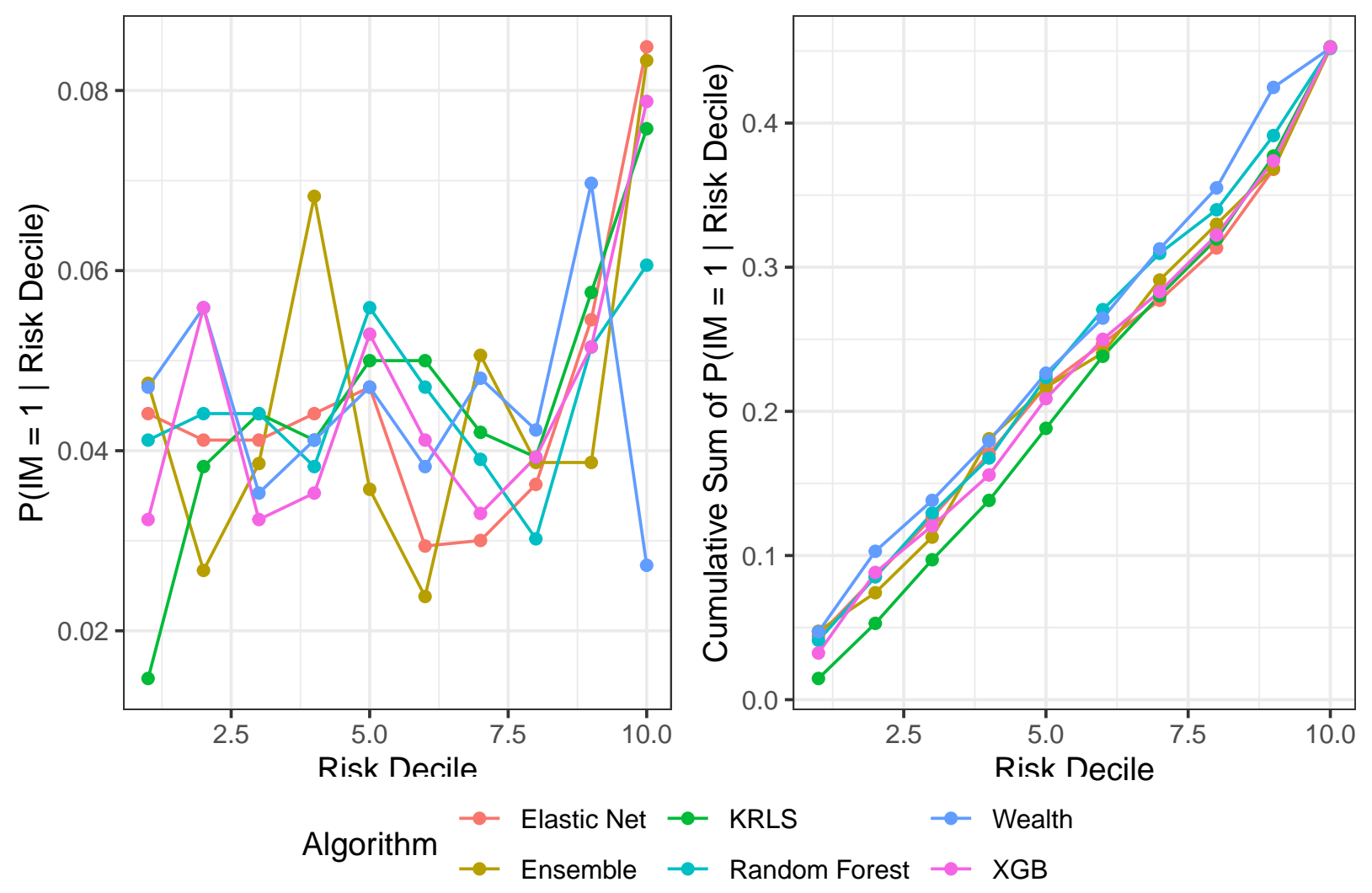

Figure 16: Probability of a mortality given that the observation is in a particular risk decile 
medRxiv preprint doi: https://doi.org/10.1101/2021.07.20.21260818; this version posted July 23, 2021. The copyright holder for this preprint (which was not certified by peer review) is the author/funder, who has granted medRxiv a license to display the preprint in perpetuity. All rights reserved. No reuse allowed without permission.
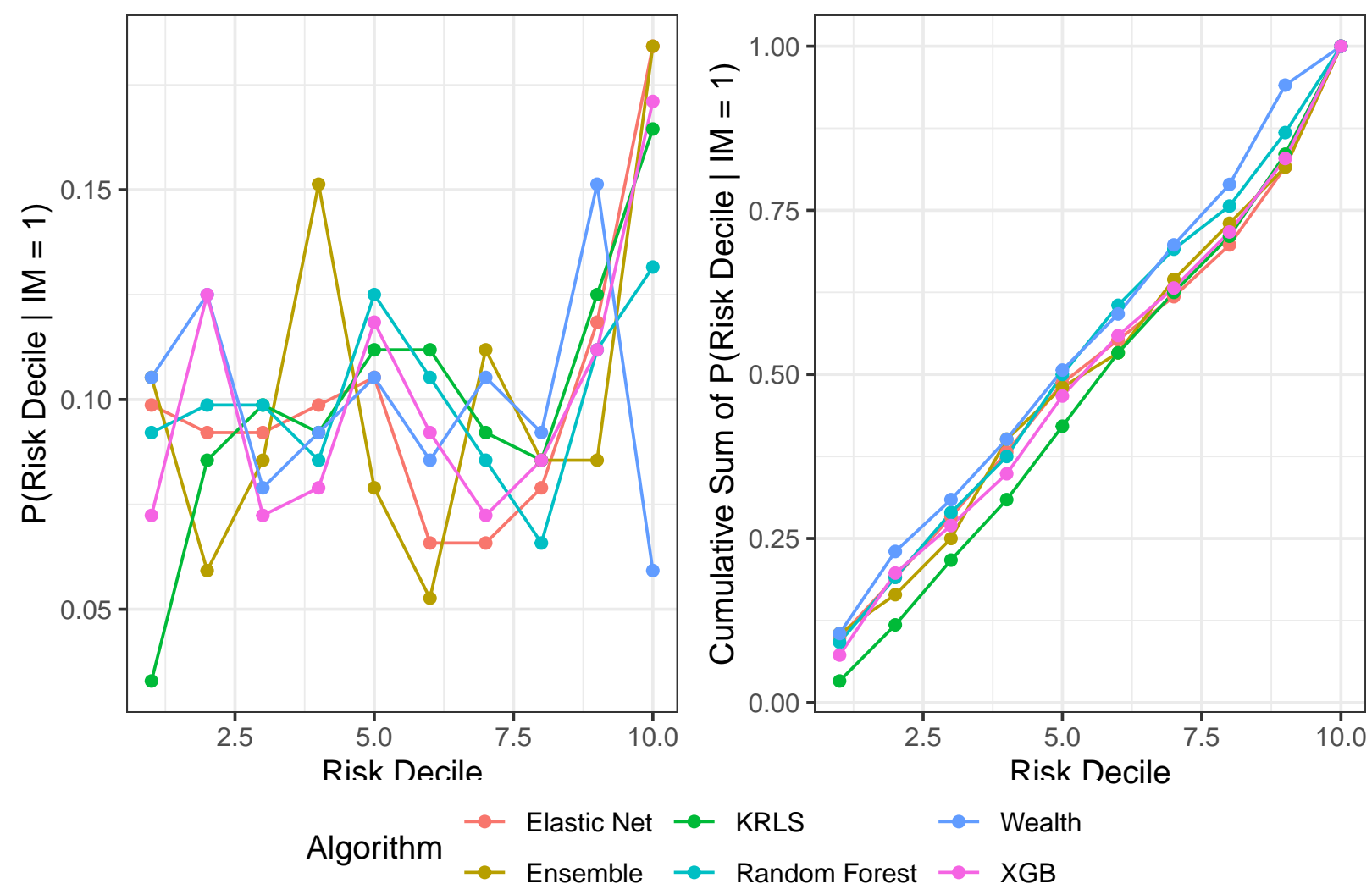

Figure 17: Probability of membership in a particular risk decile given a mortality 


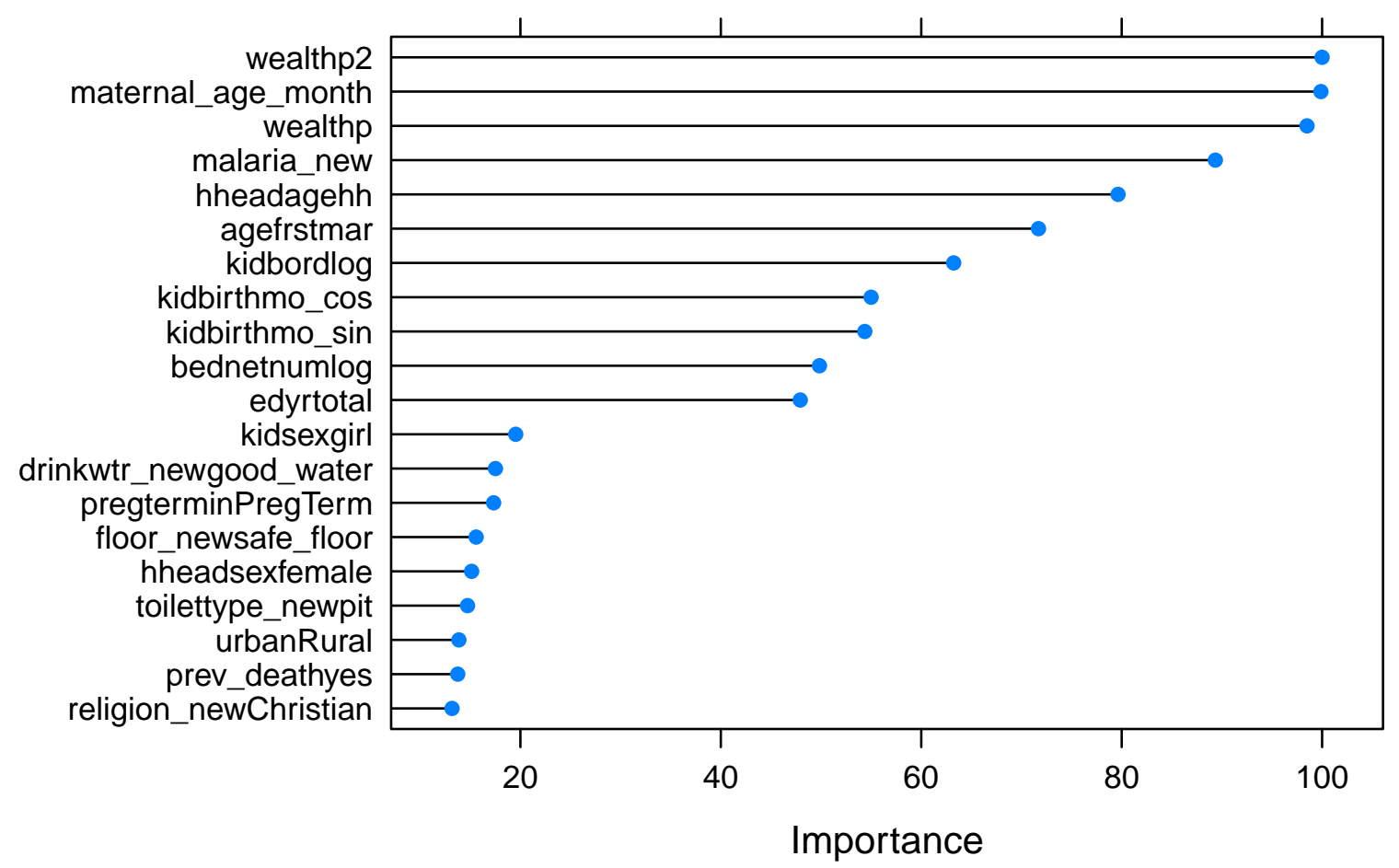

Figure 18: Variable Importance Plot generated by the Random Forest model

\section{B.7.6 Variable Importance Plot}

In this section we will show some measures to give some indication of the important variables necessary for predicting infant mortality. 
medRxiv preprint doi: https://doi.org/10.1101/2021.07.20.21260818; this version posted July 23, 2021. The copyright holder for this preprint (which was not certified by peer review) is the author/funder, who has granted medRxiv a license to display the preprint in perpetuity.

All rights reserved. No reuse allowed without permission.

\section{B.7.7 Elastic Net Coefficients}

Table 56: Standardized coefficients from the optimal elastic net model after 10-fold crossvalidation.

\begin{tabular}{|l|c|c|}
\hline & Coefficients & Standardized Coefficients \\
\hline (Intercept) & -3.1985 & -3.1985 \\
\hline pregterminPregTerm & - & - \\
\hline agefrstmar & - & $1 \mathrm{e}-04$ \\
\hline hheadagehh & - & - \\
\hline urbanRural & - & - \\
\hline kidsexgirl & - & - \\
\hline hheadsexfemale & - & - \\
\hline edyrtotal & - & - \\
\hline drinkwtr_newgood_water & - & - \\
\hline floor_newsafe_floor & - & - \\
\hline maternal_age_month & $2 \mathrm{e}-04$ & -0162 \\
\hline cookfuel_newclean_fuel & - & - \\
\hline toilettype_newpit & - & - \\
\hline toilettype_newunimproved & - & - \\
\hline religion_newChristian & - & - \\
\hline religion_newHindu & - & - \\
\hline religion_newMuslim & - & - \\
\hline religion_newOther & - & - \\
\hline bednetnumlog & - & - \\
\hline prev_deathyes & 0.4296 & - \\
\hline prev_death_fullyes & - & - \\
\hline malaria_new & - & - \\
\hline kidbordlog & - & - \\
\hline kidbirthmo_sin & - & - \\
\hline kidbirthmo_cos & - & - \\
\hline wealthp & - & - \\
\hline wealthp2 & - & - \\
\hline
\end{tabular}


medRxiv preprint doi: https://doi.org/10.1101/2021.07.20.21260818; this version posted July 23, 2021. The copyright holder for this preprint (which was not certified by peer review) is the author/funder, who has granted medRxiv a license to display the preprint in perpetuity.

All rights reserved. No reuse allowed without permission.

\section{B.8 Guinea}

This sample was taken in the year 2012. There are 5223 observations in the dataset, and 4179 observations were used for the training set. Also, 199 observations were removed due to missing values. Finally, there were 396 deaths for the full dataset, and 317 deaths in the training set. 
medRxiv preprint doi: https://doi.org/10.1101/2021.07.20.21260818; this version posted July 23, 2021. The copyright holder for this preprint (which was not certified by peer review) is the author/funder, who has granted medRxiv a license to display the preprint in perpetuity.

All rights reserved. No reuse allowed without permission. 


\section{B.8.1 Variables Used}

Table 57: Variables included in the model for Guinea

\begin{tabular}{|c|c|}
\hline Variable & Type \\
\hline mortality.under $12 \mathrm{~m}$ & categorical \\
\hline pregtermin & categorical \\
\hline agefrstmar & numeric \\
\hline hheadagehh & numeric \\
\hline urban & categorical \\
\hline kidsex & categorical \\
\hline hheadsex & categorical \\
\hline edyrtotal & numeric \\
\hline drinkwtr_new & categorical \\
\hline floor_new & categorical \\
\hline maternal_age_month & numeric \\
\hline toilettype_new & categorical \\
\hline religion_new & categorical \\
\hline bednetnumlog & numeric \\
\hline prev_death & categorical \\
\hline prev_death_full & categorical \\
\hline malaria_new & numeric \\
\hline kidbordlog & numeric \\
\hline kidbirthmo_sin & numeric \\
\hline kidbirthmo_cos & numeric \\
\hline geo_gn2012 & categorical \\
\hline wealthp & numeric \\
\hline wealthp2 & numeric \\
\hline
\end{tabular}




\section{B.8.2 Mortality Breakdown by Wealth Quintile}

\section{B.8.3 Optimal Parameters}

These are the algorithm parameters selected after cross-validation:

Table 58: Optimal Parameters for the Elastic Net algorithm

\begin{tabular}{|c|c|}
\hline alpha & lambda \\
\hline 0.4857143 & 0.0068884 \\
\hline
\end{tabular}

Table 59: Optimal Parameters for the Random Forest algorithm

\begin{tabular}{|c|}
\hline mtry \\
\hline 2 \\
\hline
\end{tabular}

Table 60: Optimal Parameters for the XGB algorithm

\begin{tabular}{|c|c|c|c|c|c|c|}
\hline nrounds & max_depth & eta & gamma & colsample_bytree & min_child_weight & subsample \\
\hline 50 & 1 & 0.3 & 0 & 0.8 & 1 & 0.875 \\
\hline
\end{tabular}

Table 61: Optimal Parameters for the KRLS algorithm

\begin{tabular}{|c|c|c|c|}
\hline loss & epsilon & b & lambda \\
\hline logistic & 0.005 & 62 & 0.000648 \\
\hline
\end{tabular}


medRxiv preprint doi: https://doi.org/10.1101/2021.07.20.21260818; this version posted July 23, 2021. The copyright holder for this preprint (which was not certified by peer review) is the author/funder, who has granted medRxiv a license to display the preprint in perpetuity.

\section{B.8.4 Table of Results}

Table 62: Manual Cross-Validation Results

\begin{tabular}{|c|c|c|c|c|c|c|c|c|c|}
\hline Algorithm & Recall 10 & Recall 20 & ROC & AUC & MRD & MRR & MSE & F1 & Efficiency Gain \\
\hline Ensemble & 0.170 & 0.318 & 0.606 & 0.122 & 0.009 & 1.134 & 0.069 & 0.918 & 1.001 \\
\hline KRLS & 0.157 & 0.274 & 0.582 & 0.116 & 0.005 & 1.071 & 0.070 & 0.918 & 0.925 \\
\hline Elastic Net & 0.157 & 0.274 & 0.576 & 0.114 & 0.005 & 1.070 & 0.070 & 0.918 & 0.926 \\
\hline Random Forest & 0.145 & 0.287 & 0.582 & 0.118 & 0.012 & 1.301 & 0.071 & 0.917 & 0.853 \\
\hline Wealth & 0.170 & 0.283 & 0.574 & 0.108 & 0.242 & 2.981 & 0.896 & 0.919 & 1.000 \\
\hline XGB & 0.139 & 0.296 & 0.599 & 0.117 & 0.013 & 1.171 & 0.070 & 0.916 & 0.819 \\
\hline
\end{tabular}

Table 63: Distribution of individuals in the top risk decile of each algorithm among each wealth decile

\begin{tabular}{l|r|r|r|r|c}
\hline Wealth Decile & Elastic Net & Ensemble & KRLS & Random Forest & XGB \\
\hline 1 & 0.298 & 0.407 & 0.249 & 0.320 & 0.427 \\
\hline 2 & 0.290 & 0.182 & 0.283 & 0.110 & 0.144 \\
\hline 3 & 0.224 & 0.144 & 0.227 & 0.068 & 0.132 \\
\hline 4 & 0.110 & 0.076 & 0.112 & 0.078 & 0.085 \\
\hline 5 & 0.041 & 0.071 & 0.061 & 0.110 & 0.063 \\
\hline 6 & 0.017 & 0.049 & 0.039 & 0.093 & 0.054 \\
\hline 7 & 0.012 & 0.034 & 0.012 & 0.067 & 0.050 \\
\hline 8 & 0.002 & 0.032 & 0.012 & 0.066 & 0.029 \\
\hline 9 & 0.000 & 0.015 & 0.000 & 0.041 & 0.012 \\
\hline 10 & 0.000 & 0.005 & 0.000 & 0.044 & 0.000 \\
\hline
\end{tabular}

\section{B.8.5 Performance Plots}

Below are various plots showing the performance for each model considered in Guinea. 
medRxiv preprint doi: https://doi.org/10.1101/2021.07.20.21260818; this version posted July 23, 2021. The copyright holder for this preprint (which was not certified by peer review) is the author/funder, who has granted medRxiv a license to display the preprint in perpetuity. All rights reserved. No reuse allowed without permission.

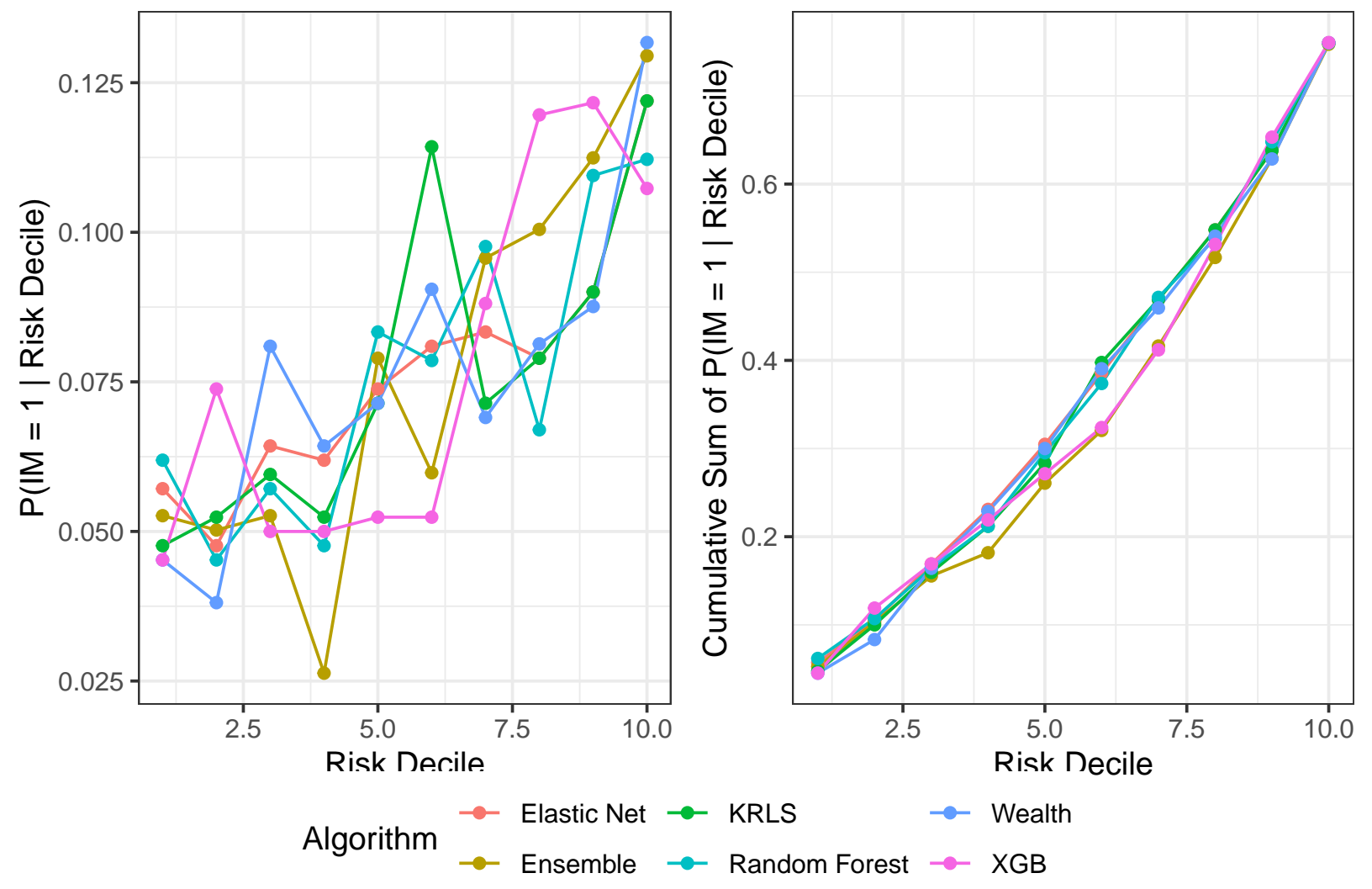

Figure 19: Probability of a mortality given that the observation is in a particular risk decile 
medRxiv preprint doi: https://doi.org/10.1101/2021.07.20.21260818; this version posted July 23, 2021. The copyright holder for this preprint (which was not certified by peer review) is the author/funder, who has granted medRxiv a license to display the preprint in perpetuity. All rights reserved. No reuse allowed without permission.
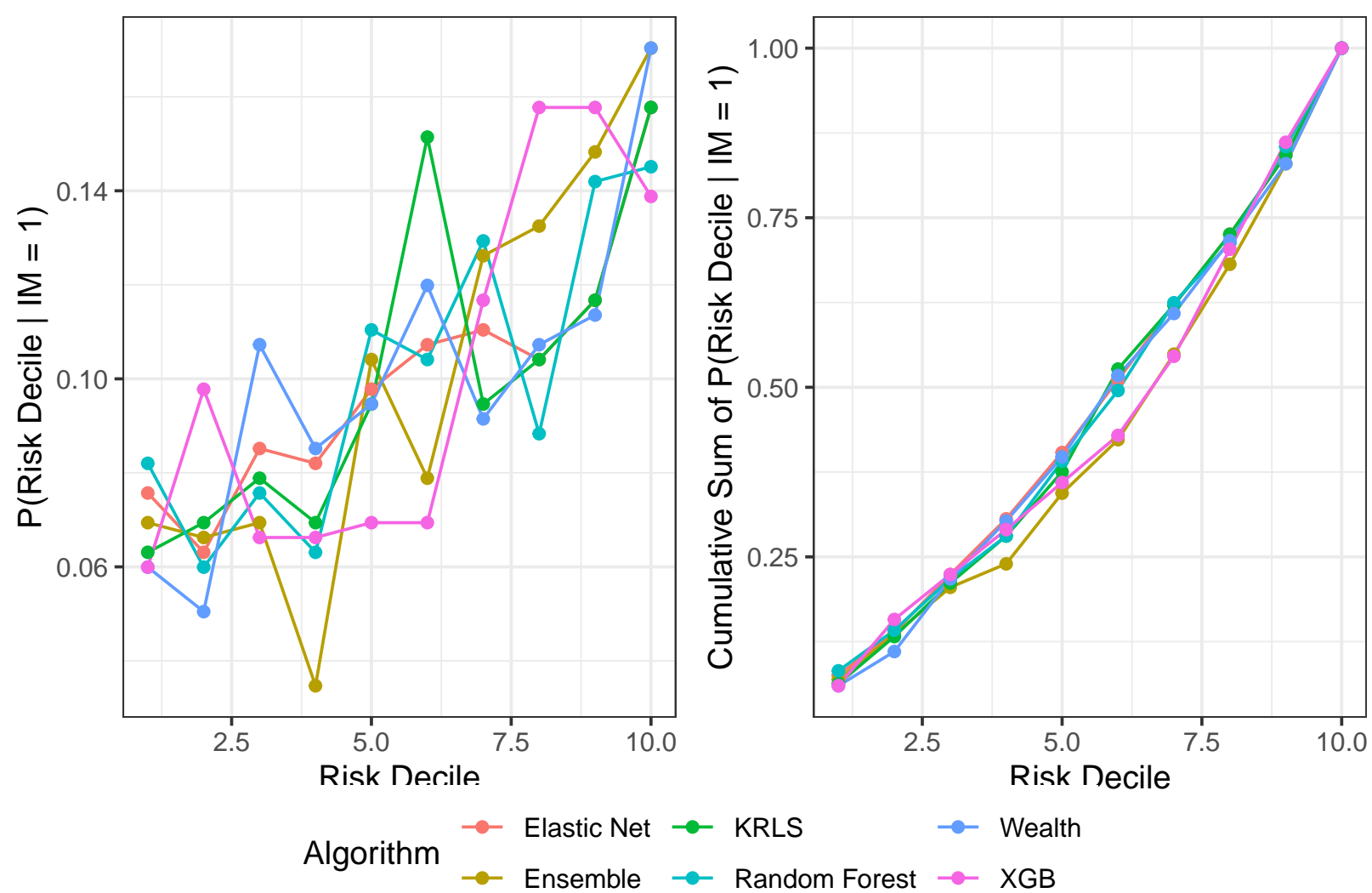

Figure 20: Probability of membership in a particular risk decile given a mortality 


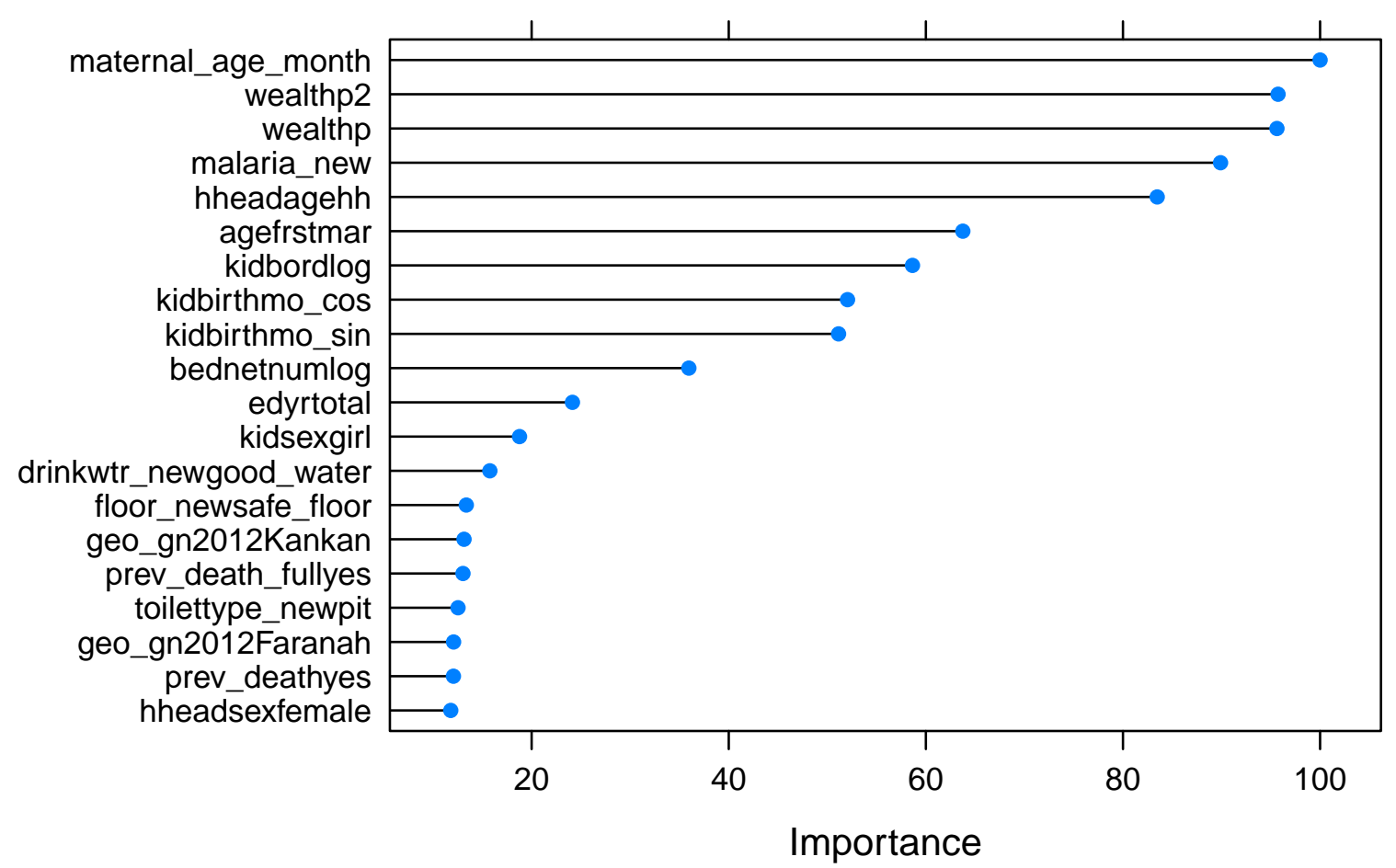

Figure 21: Variable Importance Plot generated by the Random Forest model

\section{B.8.6 Variable Importance Plot}

In this section we will show some measures to give some indication of the important variables necessary for predicting infant mortality. 
medRxiv preprint doi: https://doi.org/10.1101/2021.07.20.21260818; this version posted July 23, 2021. The copyright holder for this preprint (which was not certified by peer review) is the author/funder, who has granted medRxiv a license to display the preprint in perpetuity.

All rights reserved. No reuse allowed without permission.

\section{B.8.7 Elastic Net Coefficients}

Table 64: Standardized coefficients from the optimal elastic net model after 10-fold crossvalidation.

\begin{tabular}{|l|c|c|}
\hline & Coefficients & Standardized Coefficients \\
\hline (Intercept) & -2.1617 & -2.1617 \\
\hline pregterminPregTerm & - & - \\
\hline agefrstmar & -0.0066 & -0.0233 \\
\hline hheadagehh & - & - \\
\hline urbanRural & - & - \\
\hline kidsexgirl & - & - \\
\hline hheadsexfemale & - & - \\
\hline edyrtotal & -0.015 & -0.0466 \\
\hline drinkwtr_newgood_water & - & - \\
\hline floor_newsafe_floor & -0.0668 & -0.0334 \\
\hline maternal_age_month & $-9 \mathrm{e}-04$ & -0.0798 \\
\hline toilettype_newpit & - & - \\
\hline toilettype_newunimproved & - & - \\
\hline religion_newChristian & - & -0.1214 \\
\hline religion_newHindu & - & - \\
\hline religion_newMuslim & - & -0.0023 \\
\hline religion_newOther & - & 0.0332 \\
\hline bednetnumlog & -0.1411 & - \\
\hline prev_deathyes & 0.0065 & - \\
\hline prev_death_fullyes & 0.043 & - \\
\hline malaria_new & 0.9926 & -0.0613 \\
\hline kidbordlog & - & -0.0785 \\
\hline kidbirthmo_sin & -0.0033 & - \\
\hline kidbirthmo_cos & 0.0484 & - \\
\hline geo_gn2012Conakry & - & - \\
\hline geo_gn2012Faranah & - & - \\
\hline geo_gn2012Kankan & 0.133 & -1969 \\
\hline geo_gn2012Kindia & -0.1895 & - \\
\hline geo_gn2012Labe & - & - \\
\hline geo_gn2012Mamou & - & - \\
\hline geo_gn2012N'Zerekore & - & - \\
\hline wealthp & - & - \\
\hline wealthp2 & - & - \\
\hline
\end{tabular}


medRxiv preprint doi: https://doi.org/10.1101/2021.07.20.21260818; this version posted July 23, 2021. The copyright holder for this preprint (which was not certified by peer review) is the author/funder, who has granted medRxiv a license to display the preprint in perpetuity.

All rights reserved. No reuse allowed without permission.

\section{B.9 Kenya}

This sample was taken in the year 2014. There are 15110 observations in the dataset, and 12089 observations were used for the training set. Also, 1447 observations were removed due to missing values. Finally, there were 589 deaths for the full dataset, and 472 deaths in the training set. 


\section{B.9.1 Variables Used}

Table 65: Variables included in the model for Kenya

\begin{tabular}{|l|l|}
\hline Variable & Type \\
\hline mortality.under12m & categorical \\
\hline agefrstmar & numeric \\
\hline hheadagehh & numeric \\
\hline urban & categorical \\
\hline kidsex & categorical \\
\hline hheadsex & categorical \\
\hline edyrtotal & numeric \\
\hline drinkwtr_new & categorical \\
\hline floor_new & categorical \\
\hline maternal_age_month & numeric \\
\hline cookfuel_new & categorical \\
\hline toilettype_new & categorical \\
\hline religion_new & categorical \\
\hline bednetnumlog & numeric \\
\hline prev_death & categorical \\
\hline prev_death_full & categorical \\
\hline malaria_new & numeric \\
\hline kidbordlog & numeric \\
\hline kidbirthmo_sin & numeric \\
\hline kidbirthmo_cos & numeric \\
\hline wealthp2 & nulthp \\
\hline catic
\end{tabular}




\section{B.9.2 Mortality Breakdown by Wealth Quintile}

\section{B.9.3 Optimal Parameters}

These are the algorithm parameters selected after cross-validation:

Table 66: Optimal Parameters for the Elastic Net algorithm

\begin{tabular}{|c|c|}
\hline alpha & lambda \\
\hline 0.2285714 & 0.0082947 \\
\hline
\end{tabular}

Table 67: Optimal Parameters for the Random Forest algorithm

\begin{tabular}{|c|}
\hline mtry \\
\hline 2 \\
\hline
\end{tabular}

Table 68: Optimal Parameters for the XGB algorithm

\begin{tabular}{|c|c|c|c|c|c|c|}
\hline nrounds & max_depth & eta & gamma & colsample_bytree & min_child_weight & subsample \\
\hline 50 & 1 & 0.3 & 0 & 0.6 & 1 & 1 \\
\hline
\end{tabular}

Table 69: Optimal Parameters for the KRLS algorithm

\begin{tabular}{|c|c|c|c|}
\hline loss & epsilon & b & lambda \\
\hline logistic & 0.005 & 48 & 0.004079 \\
\hline
\end{tabular}


medRxiv preprint doi: https://doi.org/10.1101/2021.07.20.21260818; this version posted July 23, 2021. The copyright holder for this preprint (which was not certified by peer review) is the author/funder, who has granted medRxiv a license to display the preprint in perpetuity.

\section{B.9.4 Table of Results}

Table 70: Manual Cross-Validation Results

\begin{tabular}{|c|c|c|c|c|c|c|c|c|c|}
\hline Algorithm & Recall 10 & Recall 20 & ROC & AUC & MRD & MRR & MSE & F1 & Efficiency Gain \\
\hline Ensemble & 0.163 & 0.301 & 0.573 & 0.065 & 0.003 & 1.088 & 0.037 & 0.932 & 2.143 \\
\hline KRLS & 0.150 & 0.250 & 0.536 & 0.048 & 0.000 & 1.005 & 0.038 & 0.932 & 1.975 \\
\hline Elastic Net & 0.148 & 0.271 & 0.524 & 0.051 & 0.001 & 1.025 & 0.037 & 0.932 & 1.948 \\
\hline Random Forest & 0.168 & 0.282 & 0.564 & 0.066 & 0.009 & 1.408 & 0.038 & 0.933 & 2.199 \\
\hline Wealth & 0.076 & 0.167 & 0.542 & 0.039 & -0.028 & 0.882 & 1.081 & 0.929 & 1.000 \\
\hline XGB & 0.127 & 0.273 & 0.558 & 0.047 & 0.002 & 1.046 & 0.038 & 0.931 & 1.668 \\
\hline
\end{tabular}

Table 71: Distribution of individuals in the top risk decile of each algorithm among each wealth decile

\begin{tabular}{l|r|r|r|r|c}
\hline Wealth Decile & Elastic Net & Ensemble & KRLS & Random Forest & XGB \\
\hline 1 & 0.092 & 0.059 & 0.053 & 0.076 & 0.022 \\
\hline 2 & 0.105 & 0.078 & 0.079 & 0.092 & 0.044 \\
\hline 3 & 0.117 & 0.127 & 0.112 & 0.116 & 0.127 \\
\hline 4 & 0.123 & 0.108 & 0.133 & 0.092 & 0.139 \\
\hline 5 & 0.119 & 0.115 & 0.130 & 0.095 & 0.127 \\
\hline 6 & 0.125 & 0.096 & 0.131 & 0.076 & 0.127 \\
\hline 7 & 0.099 & 0.092 & 0.115 & 0.086 & 0.105 \\
\hline 8 & 0.093 & 0.111 & 0.107 & 0.115 & 0.090 \\
\hline 9 & 0.074 & 0.082 & 0.085 & 0.087 & 0.073 \\
\hline 10 & 0.052 & 0.137 & 0.054 & 0.164 & 0.143 \\
\hline
\end{tabular}

\section{B.9.5 Performance Plots}

Below are various plots showing the performance for each model considered in Kenya. 
medRxiv preprint doi: https://doi.org/10.1101/2021.07.20.21260818; this version posted July 23, 2021. The copyright holder for this preprint (which was not certified by peer review) is the author/funder, who has granted medRxiv a license to display the preprint in perpetuity. All rights reserved. No reuse allowed without permission.
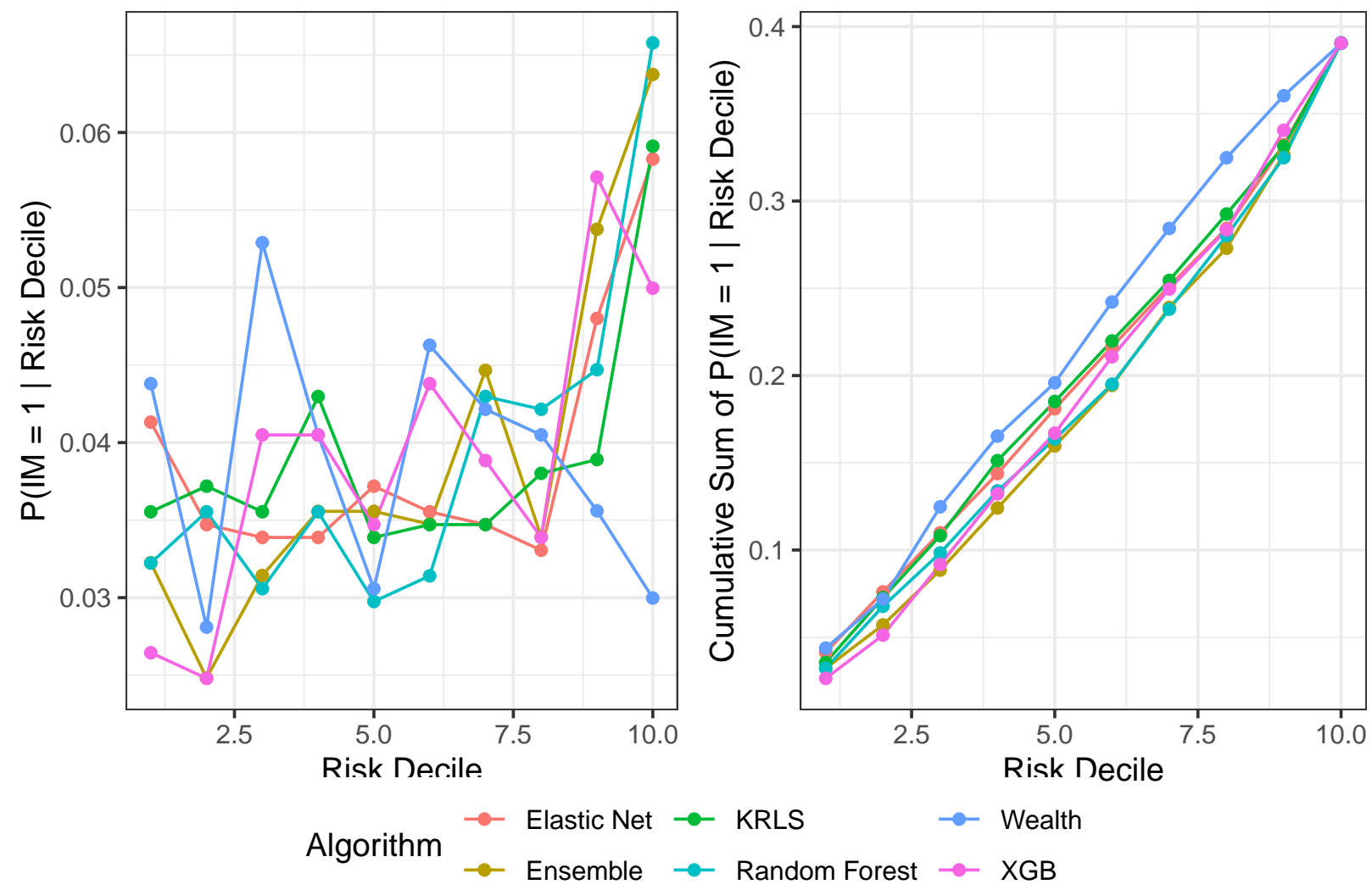

Figure 22: Probability of a mortality given that the observation is in a particular risk decile 
medRxiv preprint doi: https://doi.org/10.1101/2021.07.20.21260818; this version posted July 23, 2021. The copyright holder for this preprint (which was not certified by peer review) is the author/funder, who has granted medRxiv a license to display the preprint in perpetuity. All rights reserved. No reuse allowed without permission.
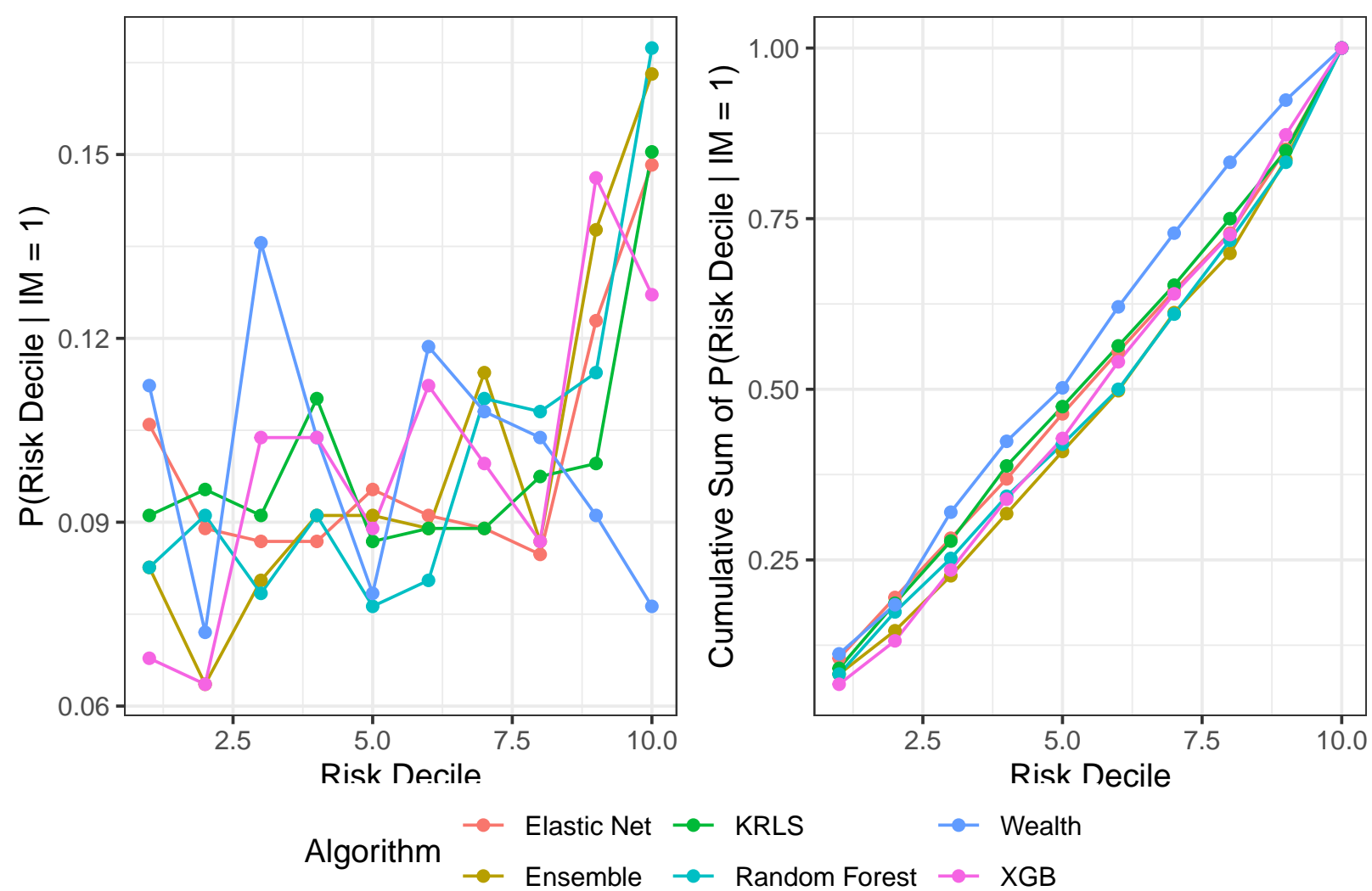

Figure 23: Probability of membership in a particular risk decile given a mortality 


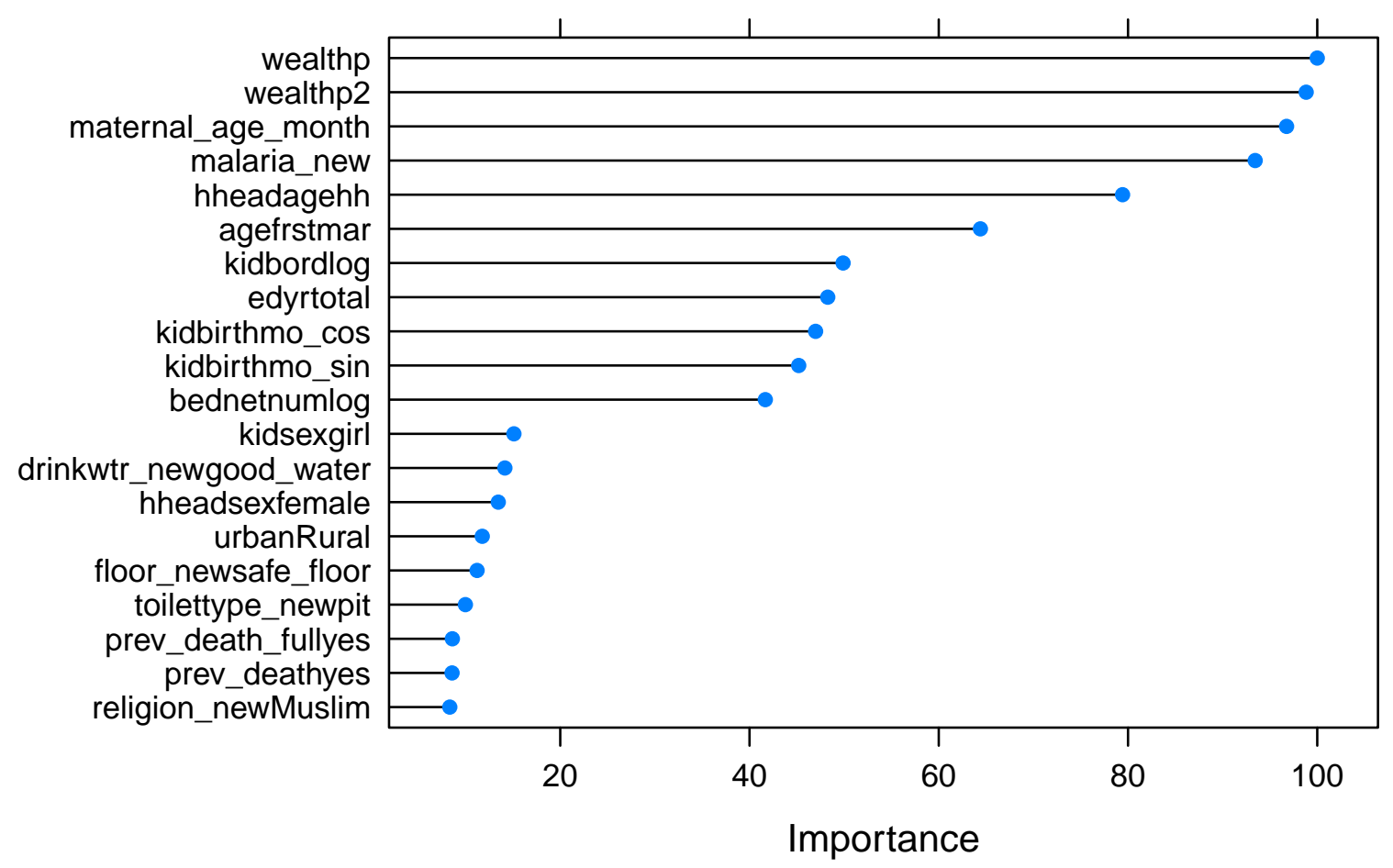

Figure 24: Variable Importance Plot generated by the Random Forest model

\section{B.9.6 Variable Importance Plot}

In this section we will show some measures to give some indication of the important variables necessary for predicting infant mortality. 
medRxiv preprint doi: https://doi.org/10.1101/2021.07.20.21260818; this version posted July 23, 2021. The copyright holder for this preprint (which was not certified by peer review) is the author/funder, who has granted medRxiv a license to display the preprint in perpetuity.

All rights reserved. No reuse allowed without permission.

\section{B.9.7 Elastic Net Coefficients}

Table 72: Standardized coefficients from the optimal elastic net model after 10-fold crossvalidation.

\begin{tabular}{|c|c|c|}
\hline & Coefficients & Standardized Coefficients \\
\hline (Intercept) & -3.2867 & -3.2867 \\
\hline agefrstmar & - & - \\
\hline hheadagehh & 0.0011 & 0.0132 \\
\hline urbanRural & - & - \\
\hline kidsexgirl & -0.0811 & -0.0406 \\
\hline hheadsexfemale & - & - \\
\hline edyrtotal & - & - \\
\hline drinkwtr_newgood_water & - & - \\
\hline floor_newsafe_floor & - & - \\
\hline maternal_age_month & - & - \\
\hline cookfuel_newclean_fuel & - & - \\
\hline toilettype_newpit & 0.0494 & 0.0234 \\
\hline toilettype_newunimproved & - & - \\
\hline religion_newChristian & - & - \\
\hline religion_newHindu & - & - \\
\hline religion_newMuslim & - & - \\
\hline religion_newOther & - & - \\
\hline bednetnumlog & -0.0235 & -0.0132 \\
\hline prev_deathyes & 0.1707 & 0.0544 \\
\hline prev_death_fullyes & 0.2008 & 0.0704 \\
\hline malaria_new & 0.0167 & 0.0019 \\
\hline kidbordlog & - & - \\
\hline kidbirthmo_sin & 0.0688 & 0.0488 \\
\hline kidbirthmo_cos & - & - \\
\hline wealthp & 0.0063 & 0.0018 \\
\hline wealthp2 & - & - \\
\hline
\end{tabular}


medRxiv preprint doi: https://doi.org/10.1101/2021.07.20.21260818; this version posted July 23, 2021. The copyright holder for this preprint (which was not certified by peer review) is the author/funder, who has granted medRxiv a license to display the preprint in perpetuity.

All rights reserved. No reuse allowed without permission.

\section{B.10 Lesotho}

This sample was taken in the year 2014. There are 2127 observations in the dataset, and 1702 observations were used for the training set. Also, 233 observations were removed due to missing values. Finally, there were 145 deaths for the full dataset, and 116 deaths in the training set. 


\section{B.10.1 Variables Used}

Table 73: Variables included in the model for Lesotho

\begin{tabular}{|c|c|}
\hline Variable & Type \\
\hline mortality.under $12 \mathrm{~m}$ & categorical \\
\hline pregtermin & categorical \\
\hline agefrstmar & numeric \\
\hline hheadagehh & numeric \\
\hline urban & categorical \\
\hline kidsex & categorical \\
\hline hheadsex & categorical \\
\hline edyrtotal & numeric \\
\hline drinkwtr_new & categorical \\
\hline floor_new & categorical \\
\hline maternal_age_month & numeric \\
\hline cookfuel_new & categorical \\
\hline toilettype_new & categorical \\
\hline religion_new & categorical \\
\hline prev_death & categorical \\
\hline prev_death_full & categorical \\
\hline kidbordlog & numeric \\
\hline kidbirthmo_sin & numeric \\
\hline kidbirthmo_cos & numeric \\
\hline geo_ls2014 & categorical \\
\hline wealthp & numeric \\
\hline wealthp2 & numeric \\
\hline
\end{tabular}




\section{B.10.2 Mortality Breakdown by Wealth Quintile}

\section{B.10.3 Optimal Parameters}

These are the algorithm parameters selected after cross-validation:

Table 74: Optimal Parameters for the Elastic Net algorithm

\begin{tabular}{|c|c|}
\hline alpha & lambda \\
\hline 0.6785714 & 0.019543 \\
\hline
\end{tabular}

Table 75: Optimal Parameters for the Random Forest algorithm

\begin{tabular}{|c|}
\hline mtry \\
\hline 2 \\
\hline
\end{tabular}

Table 76: Optimal Parameters for the XGB algorithm

\begin{tabular}{|c|c|c|c|c|c|c|}
\hline nrounds & max_depth & eta & gamma & colsample_bytree & min_child_weight & subsample \\
\hline 50 & 1 & 0.3 & 0 & 0.8 & 1 & 1 \\
\hline
\end{tabular}

Table 77: Optimal Parameters for the KRLS algorithm

\begin{tabular}{|c|c|c|c|}
\hline loss & epsilon & $\mathbf{b}$ & lambda \\
\hline logistic & 0.005 & 64 & 0.001915 \\
\hline
\end{tabular}


medRxiv preprint doi: https://doi.org/10.1101/2021.07.20.21260818; this version posted July 23, 2021. The copyright holder for this preprint (which was not certified by peer review) is the author/funder, who has granted medRxiv a license to display the preprint in perpetuity.

\section{B.10.4 Table of Results}

Table 78: Manual Cross-Validation Results

\begin{tabular}{|c|c|c|c|c|c|c|c|c|c|}
\hline Algorithm & Recall 10 & Recall 20 & ROC & AUC & MRD & MRR & MSE & F1 & Efficiency Gain \\
\hline Ensemble & 0.138 & 0.267 & 0.586 & 0.112 & 0.005 & 1.076 & 0.063 & 0.918 & 2.022 \\
\hline KRLS & 0.145 & 0.249 & 0.548 & 0.089 & 0.001 & 1.017 & 0.063 & 0.920 & 2.133 \\
\hline Elastic Net & 0.094 & 0.214 & 0.575 & 0.083 & 0.001 & 1.011 & 0.063 & 0.916 & 1.378 \\
\hline Random Forest & 0.162 & 0.317 & 0.582 & 0.128 & 0.013 & 1.344 & 0.064 & 0.921 & 2.378 \\
\hline Wealth & 0.068 & 0.137 & 0.583 & 0.079 & -0.026 & 0.859 & 0.993 & 0.914 & 1.000 \\
\hline XGB & 0.077 & 0.181 & 0.522 & 0.087 & 0.003 & 1.047 & 0.064 & 0.915 & 1.133 \\
\hline
\end{tabular}

Table 79: Distribution of individuals in the top risk decile of each algorithm among each wealth decile

\begin{tabular}{l|r|r|r|r|c}
\hline Wealth Decile & Elastic Net & Ensemble & KRLS & Random Forest & XGB \\
\hline 1 & 0.268 & 0.054 & 0.060 & 0.089 & 0.054 \\
\hline 2 & 0.124 & 0.024 & 0.053 & 0.065 & 0.000 \\
\hline 3 & 0.214 & 0.137 & 0.143 & 0.125 & 0.113 \\
\hline 4 & 0.113 & 0.065 & 0.071 & 0.060 & 0.024 \\
\hline 5 & 0.071 & 0.060 & 0.107 & 0.071 & 0.036 \\
\hline 6 & 0.048 & 0.065 & 0.125 & 0.083 & 0.042 \\
\hline 7 & 0.076 & 0.241 & 0.176 & 0.147 & 0.282 \\
\hline 8 & 0.054 & 0.185 & 0.131 & 0.161 & 0.238 \\
\hline 9 & 0.012 & 0.101 & 0.071 & 0.089 & 0.137 \\
\hline 10 & 0.018 & 0.077 & 0.060 & 0.107 & 0.071 \\
\hline
\end{tabular}

\section{B.10.5 Performance Plots}

Below are various plots showing the performance for each model considered in Lesotho. 
medRxiv preprint doi: https://doi.org/10.1101/2021.07.20.21260818; this version posted July 23, 2021. The copyright holder for this preprint (which was not certified by peer review) is the author/funder, who has granted medRxiv a license to display the preprint in perpetuity. All rights reserved. No reuse allowed without permission.

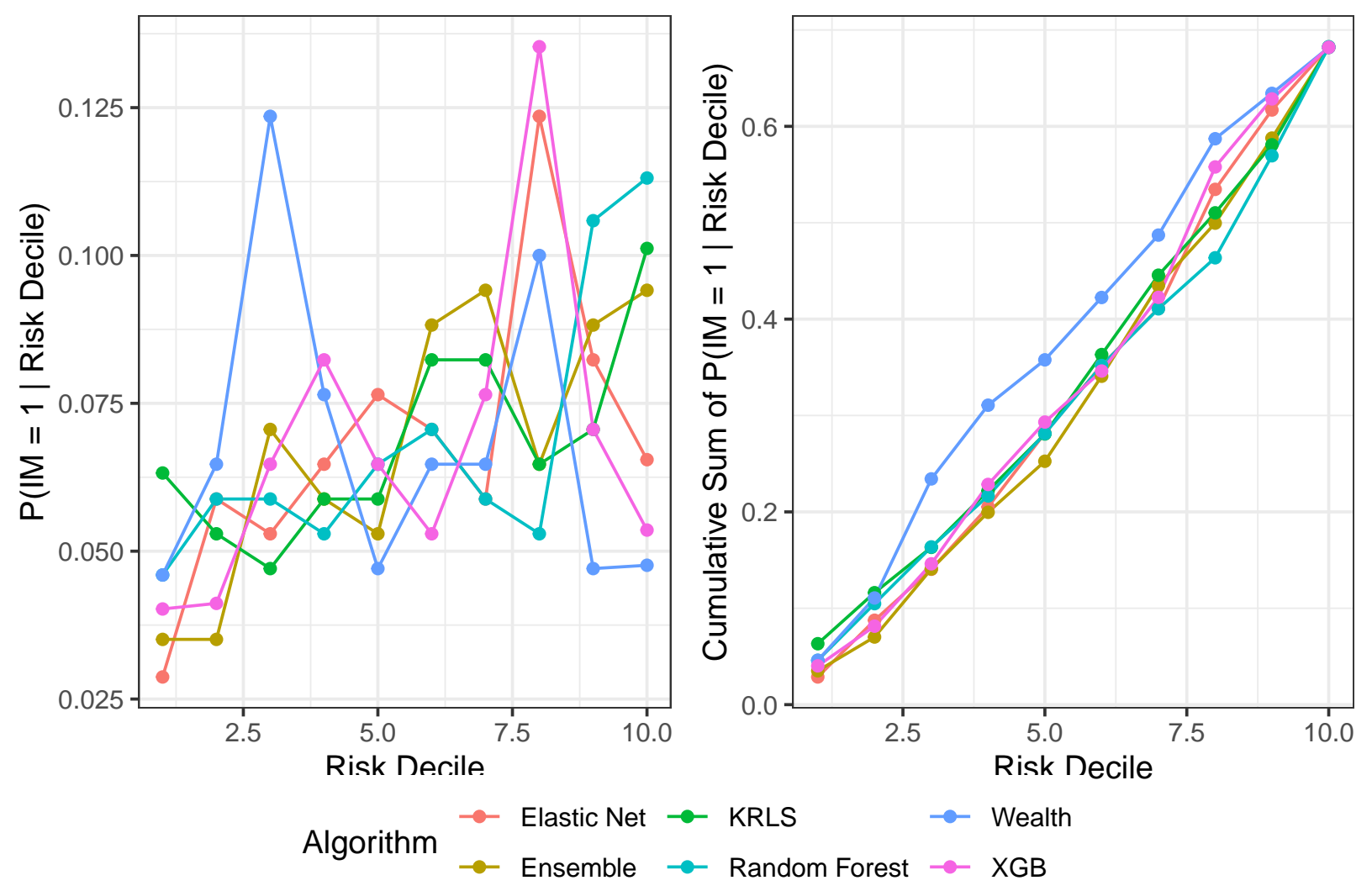

Figure 25: Probability of a mortality given that the observation is in a particular risk decile 
medRxiv preprint doi: https://doi.org/10.1101/2021.07.20.21260818; this version posted July 23, 2021. The copyright holder for this preprint (which was not certified by peer review) is the author/funder, who has granted medRxiv a license to display the preprint in perpetuity. All rights reserved. No reuse allowed without permission.
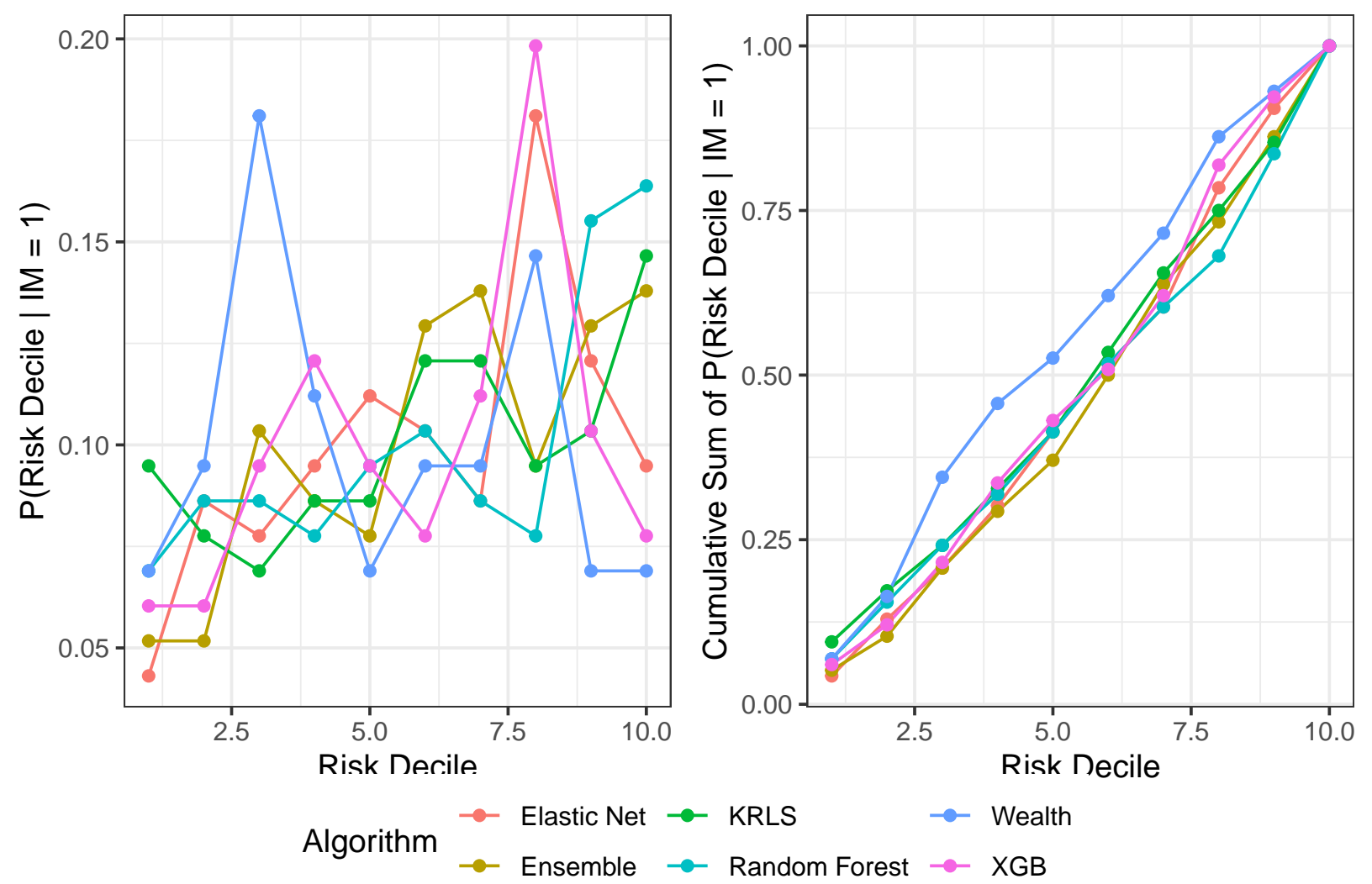

Figure 26: Probability of membership in a particular risk decile given a mortality 


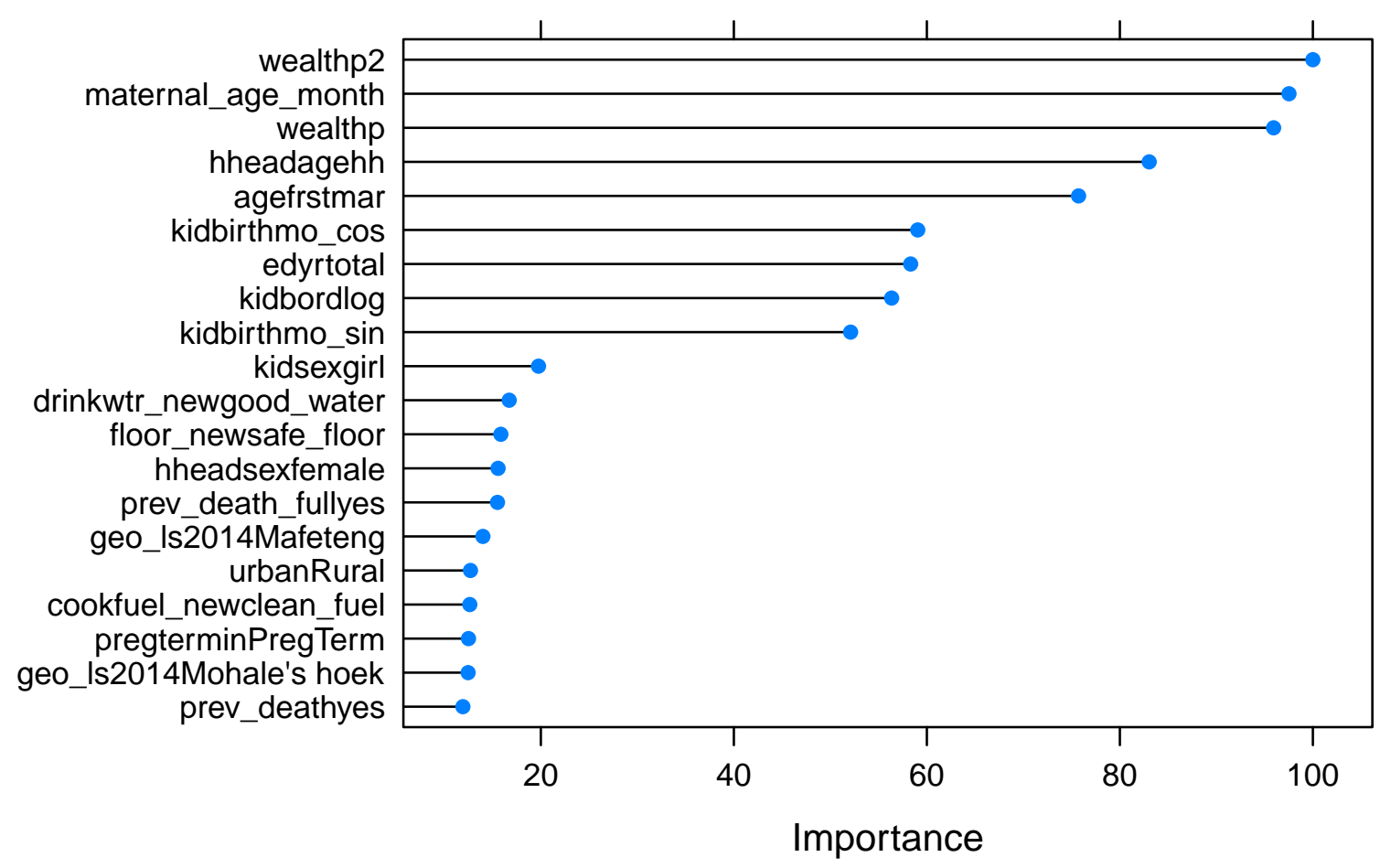

Figure 27: Variable Importance Plot generated by the Random Forest model

\section{B.10.6 Variable Importance Plot}

In this section we will show some measures to give some indication of the important variables necessary for predicting infant mortality. 
medRxiv preprint doi: https://doi.org/10.1101/2021.07.20.21260818; this version posted July 23, 2021. The copyright holder for this preprint (which was not certified by peer review) is the author/funder, who has granted medRxiv a license to display the preprint in perpetuity.

All rights reserved. No reuse allowed without permission.

\section{B.10.7 Elastic Net Coefficients}

Table 80: Standardized coefficients from the optimal elastic net model after 10-fold crossvalidation.

\begin{tabular}{|l|c|c|}
\hline & Coefficients & Standardized Coefficients \\
\hline (Intercept) & -2.439 & -2.439 \\
\hline pregterminPregTerm & - & - \\
\hline agefrstmar & - & - \\
\hline hheadagehh & - & - \\
\hline urbanRural & - & - \\
\hline kidsexgirl & - & - \\
\hline hheadsexfemale & - & - \\
\hline edyrtotal & -0.0208 & -0.06 \\
\hline drinkwtr_newgood_water & - & - \\
\hline floor_newsafe_floor & - & - \\
\hline maternal_age_month & - & - \\
\hline cookfuel_newclean_fuel & - & - \\
\hline toilettype_newpit & - & - \\
\hline toilettype_newunimproved & - & - \\
\hline religion_newChristian & - & - \\
\hline religion_newHindu & - & - \\
\hline religion_newMuslim & - & - \\
\hline religion_newOther & - & - \\
\hline prev_deathyes & - & - \\
\hline prev_death_fullyes & - & - \\
\hline kidbordlog & - & - \\
\hline kidbirthmo_sin & - & - \\
\hline kidbirthmo_cos & - & - \\
\hline geo_ls2014Leribe & - & - \\
\hline geo_ls2014Berea & - & - \\
\hline geo_ls2014Maseru & - & - \\
\hline geo_ls2014Mafeteng & - & - \\
\hline geo_ls2014Mohale's hoek & - & - \\
\hline geo_ls2014Quthing & - & - \\
\hline geo_ls2014Qacha's-nek & - & - \\
\hline geo_ls2014Mokhotlong & - & - \\
\hline geo_ls2014Thaba Tseka & - & - \\
\hline wealthp & - & - \\
\hline wealthp2 & - & - \\
\hline
\end{tabular}


medRxiv preprint doi: https://doi.org/10.1101/2021.07.20.21260818; this version posted July 23, 2021. The copyright holder for this preprint (which was not certified by peer review) is the author/funder, who has granted medRxiv a license to display the preprint in perpetuity.

All rights reserved. No reuse allowed without permission.

\section{B.11 Madagascar}

This sample was taken in the year 2008. There are 9229 observations in the dataset, and 7384 observations were used for the training set. Also, 428 observations were removed due to missing values. Finally, there were 459 deaths for the full dataset, and 368 deaths in the training set. 
medRxiv preprint doi: https://doi.org/10.1101/2021.07.20.21260818; this version posted July 23, 2021. The copyright holder for this preprint (which was not certified by peer review) is the author/funder, who has granted medRxiv a license to display the preprint in perpetuity.

All rights reserved. No reuse allowed without permission. 


\section{B.11.1 Variables Used}

Table 81: Variables included in the model for Madagascar

\begin{tabular}{|c|c|}
\hline Variable & Type \\
\hline mortality.under $12 \mathrm{~m}$ & categorical \\
\hline pregtermin & categorical \\
\hline agefrstmar & numeric \\
\hline hheadagehh & numeric \\
\hline urban & categorical \\
\hline kidsex & categorical \\
\hline hheadsex & categorical \\
\hline edyrtotal & numeric \\
\hline drinkwtr_new & categorical \\
\hline floor_new & categorical \\
\hline maternal_age_month & numeric \\
\hline toilettype_new & categorical \\
\hline religion_new & categorical \\
\hline bednetnumlog & numeric \\
\hline prev_death & categorical \\
\hline prev_death_full & categorical \\
\hline malaria_new & numeric \\
\hline kidbordlog & numeric \\
\hline kidbirthmo_sin & numeric \\
\hline kidbirthmo_cos & numeric \\
\hline geo_mg2008 & categorical \\
\hline wealthp & numeric \\
\hline wealthp2 & numeric \\
\hline
\end{tabular}




\section{B.11.2 Mortality Breakdown by Wealth Quintile}

\section{B.11.3 Optimal Parameters}

These are the algorithm parameters selected after cross-validation:

Table 82: Optimal Parameters for the Elastic Net algorithm

\begin{tabular}{|c|c|}
\hline alpha & lambda \\
\hline 0.8714286 & 0.0026119 \\
\hline
\end{tabular}

Table 83: Optimal Parameters for the Random Forest algorithm

\begin{tabular}{|c|}
\hline mtry \\
\hline 13 \\
\hline
\end{tabular}

Table 84: Optimal Parameters for the XGB algorithm

\begin{tabular}{|c|c|c|c|c|c|c|}
\hline nrounds & max_depth & eta & gamma & colsample_bytree & min_child_weight & subsample \\
\hline 50 & 1 & 0.3 & 0 & 0.8 & 1 & 0.875 \\
\hline
\end{tabular}

Table 85: Optimal Parameters for the KRLS algorithm

\begin{tabular}{|c|c|c|c|}
\hline loss & epsilon & $\mathbf{b}$ & lambda \\
\hline logistic & 0.005 & 90 & 0.000118 \\
\hline
\end{tabular}


medRxiv preprint doi: https://doi.org/10.1101/2021.07.20.21260818; this version posted July 23, 2021. The copyright holder for this preprint (which was not certified by peer review) is the author/funder, who has granted medRxiv a license to display the preprint in perpetuity.

\section{B.11.4 Table of Results}

Table 86: Manual Cross-Validation Results

\begin{tabular}{|c|c|c|c|c|c|c|c|c|c|}
\hline Algorithm & Recall 10 & Recall 20 & ROC & AUC & MRD & MRR & MSE & F1 & Efficiency Gain \\
\hline Ensemble & 0.274 & 0.424 & 0.656 & 0.148 & 0.024 & 1.473 & 0.046 & 0.934 & 2.652 \\
\hline KRLS & 0.250 & 0.391 & 0.642 & 0.100 & 0.014 & 1.288 & 0.047 & 0.933 & 2.417 \\
\hline Elastic Net & 0.242 & 0.378 & 0.629 & 0.097 & 0.013 & 1.270 & 0.047 & 0.933 & 2.339 \\
\hline Random Forest & 0.236 & 0.361 & 0.635 & 0.141 & 0.052 & 1.933 & 0.047 & 0.932 & 2.285 \\
\hline Wealth & 0.103 & 0.223 & 0.549 & 0.053 & 0.126 & 1.532 & 0.795 & 0.925 & 1.000 \\
\hline XGB & 0.245 & 0.391 & 0.653 & 0.098 & 0.016 & 1.338 & 0.047 & 0.933 & 2.364 \\
\hline
\end{tabular}

Table 87: Distribution of individuals in the top risk decile of each algorithm among each wealth decile

\begin{tabular}{l|r|r|r|r|c}
\hline Wealth Decile & Elastic Net & Ensemble & KRLS & Random Forest & XGB \\
\hline 1 & 0.132 & 0.134 & 0.126 & 0.138 & 0.107 \\
\hline 2 & 0.146 & 0.152 & 0.156 & 0.141 & 0.157 \\
\hline 3 & 0.129 & 0.129 & 0.136 & 0.101 & 0.112 \\
\hline 4 & 0.100 & 0.115 & 0.108 & 0.099 & 0.126 \\
\hline 5 & 0.123 & 0.123 & 0.136 & 0.092 & 0.129 \\
\hline 6 & 0.111 & 0.100 & 0.115 & 0.085 & 0.112 \\
\hline 7 & 0.103 & 0.092 & 0.093 & 0.107 & 0.106 \\
\hline 8 & 0.079 & 0.073 & 0.077 & 0.086 & 0.088 \\
\hline 9 & 0.049 & 0.049 & 0.041 & 0.074 & 0.045 \\
\hline 10 & 0.025 & 0.041 & 0.010 & 0.074 & 0.015 \\
\hline
\end{tabular}

\section{B.11.5 Performance Plots}

Below are various plots showing the performance for each model considered in Madagascar. 
medRxiv preprint doi: https://doi.org/10.1101/2021.07.20.21260818; this version posted July 23, 2021. The copyright holder for this preprint (which was not certified by peer review) is the author/funder, who has granted medRxiv a license to display the preprint in perpetuity. All rights reserved. No reuse allowed without permission.

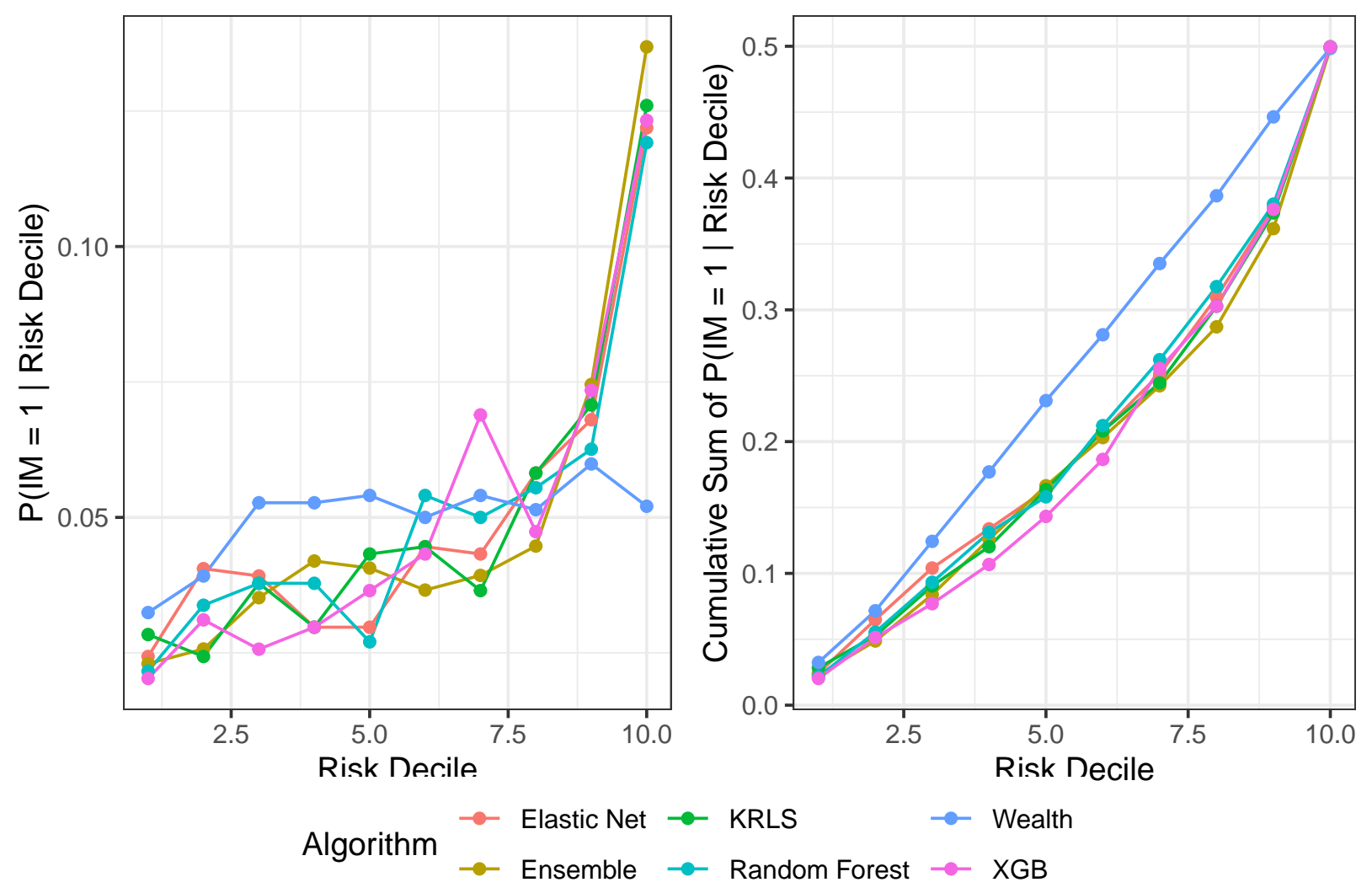

Figure 28: Probability of a mortality given that the observation is in a particular risk decile 
medRxiv preprint doi: https://doi.org/10.1101/2021.07.20.21260818; this version posted July 23, 2021. The copyright holder for this preprint (which was not certified by peer review) is the author/funder, who has granted medRxiv a license to display the preprint in perpetuity. All rights reserved. No reuse allowed without permission.
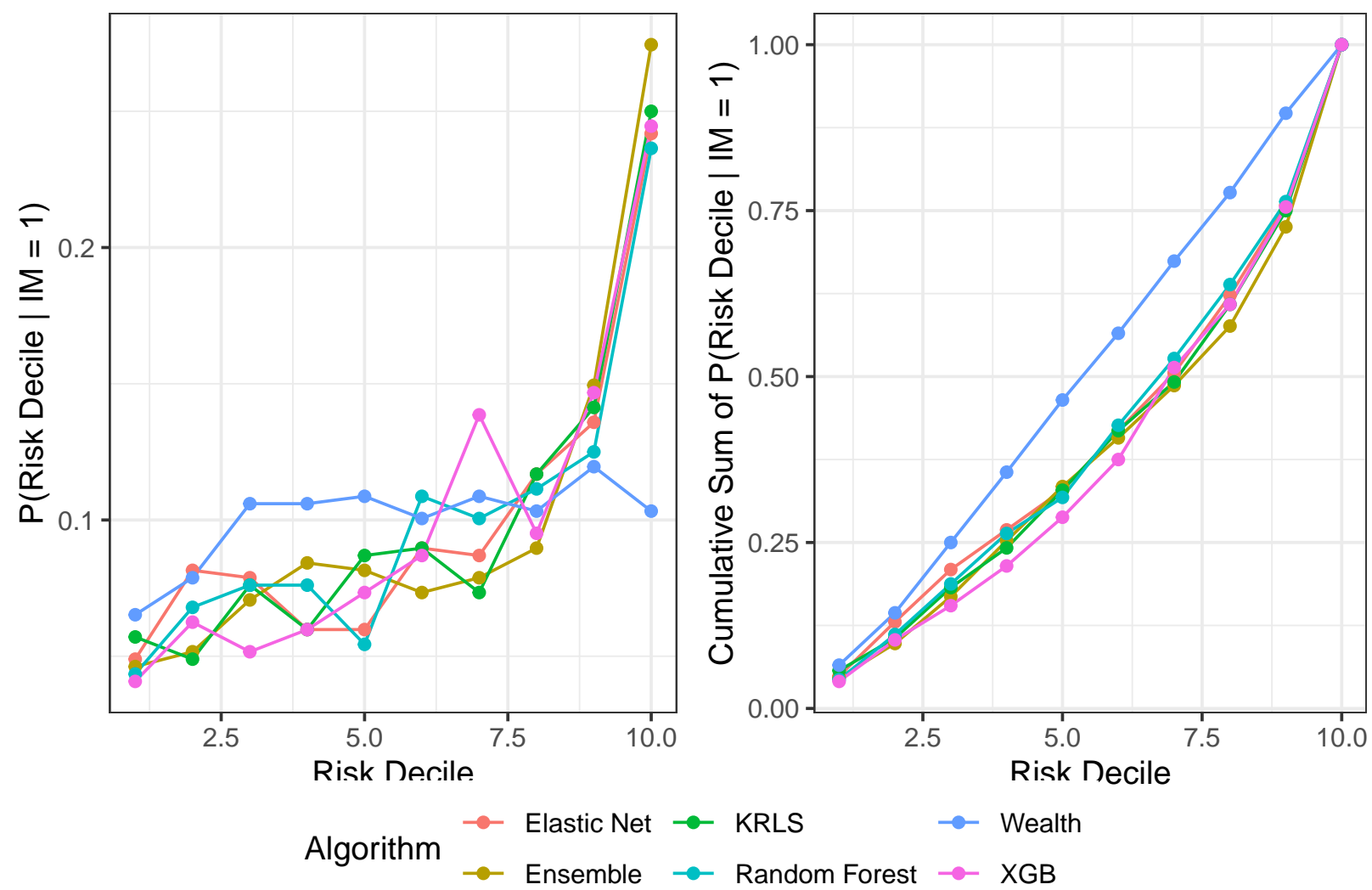

Figure 29: Probability of membership in a particular risk decile given a mortality 


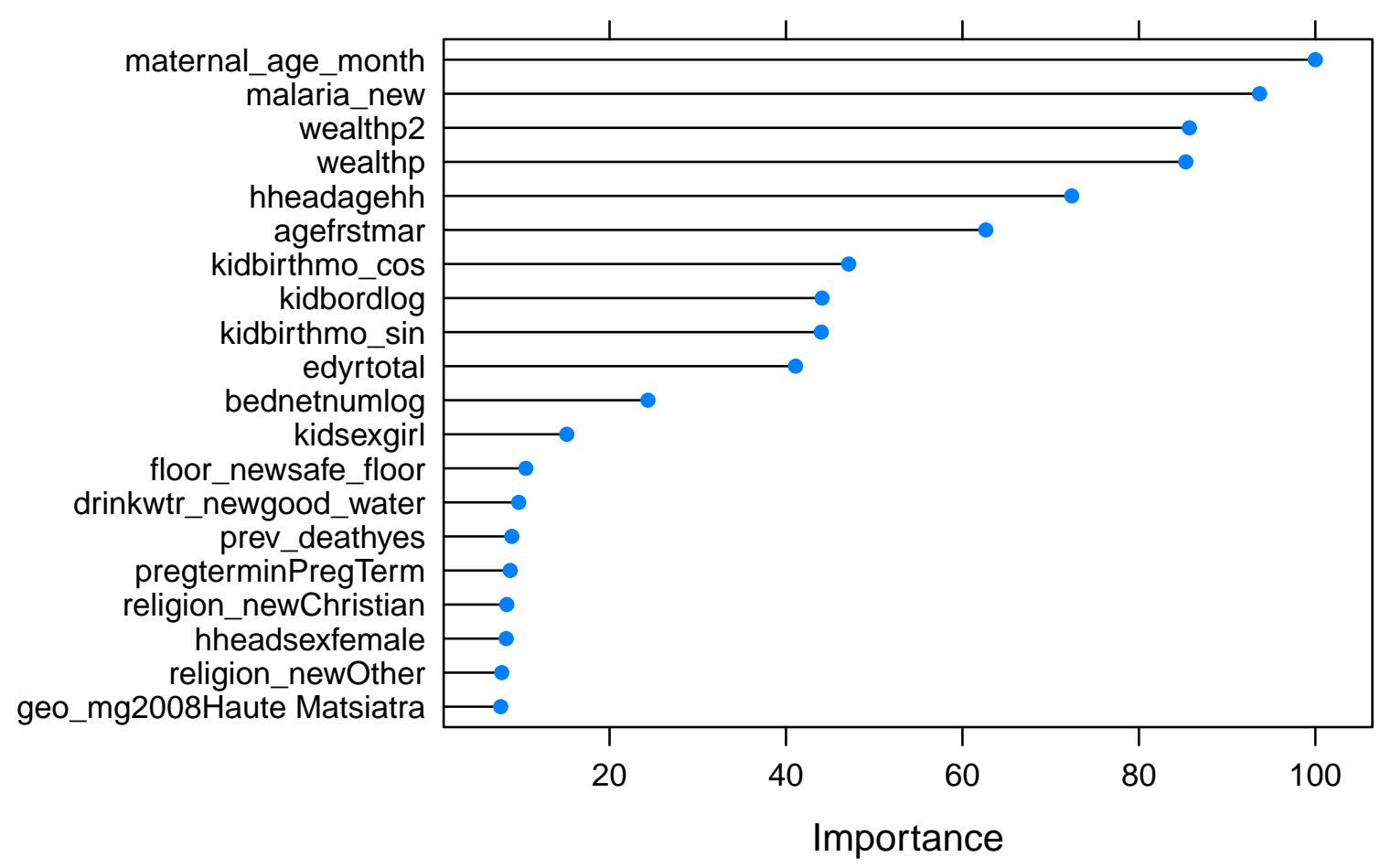

Figure 30: Variable Importance Plot generated by the Random Forest model

\section{B.11.6 Variable Importance Plot}

In this section we will show some measures to give some indication of the important variables necessary for predicting infant mortality. 


\section{B.11.7 Elastic Net Coefficients}

Table 88: Standardized coefficients from the optimal elastic net model after 10-fold crossvalidation.

\begin{tabular}{|c|c|c|}
\hline & Coefficients & Standardized Coefficients \\
\hline (Intercept) & -3.333 & -3.333 \\
\hline pregterminPregTerm & - & - \\
\hline agefrstmar & 0.0054 & 0.021 \\
\hline hheadagehh & $3 e-04$ & 0.0042 \\
\hline urbanRural & 0.0413 & 0.0157 \\
\hline kidsexgirl & -0.0498 & -0.0249 \\
\hline hheadsexfemale & - & - \\
\hline edyrtotal & -0.0014 & -0.0047 \\
\hline drinkwtr_newgood_water & - & - \\
\hline floor_newsafe_floor & -0.0063 & -0.003 \\
\hline maternal_age_month & - & - \\
\hline toilettype_newpit & - & - \\
\hline toilettype_newunimproved & 0.2149 & 0.1065 \\
\hline religion_newChristian & 0.1559 & 0.0744 \\
\hline religion_newHindu & - & - \\
\hline religion_newMuslim & - & - \\
\hline religion_newOther & - & - \\
\hline bednetnumlog & -0.2581 & -0.1232 \\
\hline prev_deathyes & 0.9229 & 0.3388 \\
\hline prev_death_fullyes & - & - \\
\hline malaria_new & - & - \\
\hline kidbordlog & -0.0541 & -0.039 \\
\hline kidbirthmo_sin & 0.1048 & 0.0738 \\
\hline kidbirthmo_cos & -0.0615 & -0.0436 \\
\hline geo_mg2008Vakinankaratra & - & - \\
\hline geo_mg2008Itasy & - & - \\
\hline geo_mg2008Bongolava & - & - \\
\hline geo_mg2008Haute Matsiatra & 0.3168 & 0.071 \\
\hline geo_mg2008Anamoroni'i Mania & - & - \\
\hline geo_mg2008Vatovavy Fitovinany & 0.3908 & 0.088 \\
\hline geo_mg2008Ihorombe & - & - \\
\hline geo_mg2008Atsimo Atsinanana & - & - \\
\hline geo_mg2008Atsinanana & - & - \\
\hline geo_mg2008Analanjirofo & - & - \\
\hline geo_mg2008Alaotra Mangoro & - & - \\
\hline geo_mg2008Boeny & 0.3558 & 0.0689 \\
\hline geo_mg2008Sofia & -0.1246 & -0.0253 \\
\hline geo_mg2008Betsiboka & - & - \\
\hline geo_mg2008Melaky & -0.4814 & -0.085 \\
\hline
\end{tabular}


medRxiv preprint doi: https://doi.org/10.1101/2021.07.20.21260818; this version posted July 23, 2021. The copyright holder for this preprint (which was not certified by peer review) is the author/funder, who has granted medRxiv a license to display the preprint in perpetuity.

All rights reserved. No reuse allowed without permission.

Table 88: Standardized coefficients from the optimal elastic net model after 10-fold crossvalidation. (continued)

\begin{tabular}{|l|c|c|}
\hline & Coefficients & Standardized Coefficients \\
\hline geo_mg2008Atsimo Andrefana & -0.0529 & -0.0113 \\
\hline geo_mg2008Androy & -0.0196 & -0.0043 \\
\hline geo_mg2008Anosy & 0.081 & 0.0159 \\
\hline geo_mg2008Menabe & - & - \\
\hline geo_mg2008Diana & -0.1846 & -0.0286 \\
\hline geo_mg2008Sava & - & - \\
\hline wealthp & - & - \\
\hline wealthp2 & - & - \\
\hline
\end{tabular}


medRxiv preprint doi: https://doi.org/10.1101/2021.07.20.21260818; this version posted July 23, 2021. The copyright holder for this preprint (which was not certified by peer review) is the author/funder, who has granted medRxiv a license to display the preprint in perpetuity.

All rights reserved. No reuse allowed without permission.

\section{B.12 Malawi}

This sample was taken in the year 2016. There are 13231 observations in the dataset, and 10586 observations were used for the training set. Also, 402 observations were removed due to missing values. Finally, there were 563 deaths for the full dataset, and 451 deaths in the training set. 
medRxiv preprint doi: https://doi.org/10.1101/2021.07.20.21260818; this version posted July 23, 2021. The copyright holder for this preprint (which was not certified by peer review) is the author/funder, who has granted medRxiv a license to display the preprint in perpetuity.

All rights reserved. No reuse allowed without permission. 


\section{B.12.1 Variables Used}

Table 89: Variables included in the model for Malawi

\begin{tabular}{|c|c|}
\hline Variable & Type \\
\hline mortality.under $12 \mathrm{~m}$ & categorical \\
\hline pregtermin & categorical \\
\hline agefrstmar & numeric \\
\hline hheadagehh & numeric \\
\hline urban & categorical \\
\hline kidsex & categorical \\
\hline hheadsex & categorical \\
\hline edyrtotal & numeric \\
\hline drinkwtr_new & categorical \\
\hline floor_new & categorical \\
\hline maternal_age_month & numeric \\
\hline cookfuel_new & categorical \\
\hline toilettype_new & categorical \\
\hline religion_new & categorical \\
\hline bednetnumlog & numeric \\
\hline prev_death & categorical \\
\hline prev_death_full & categorical \\
\hline kidbordlog & numeric \\
\hline kidbirthmo_sin & numeric \\
\hline kidbirthmo_cos & numeric \\
\hline geo_mw2016 & categorical \\
\hline wealthp & numeric \\
\hline wealthp2 & numeric \\
\hline
\end{tabular}




\section{B.12.2 Mortality Breakdown by Wealth Quintile}

\section{B.12.3 Optimal Parameters}

These are the algorithm parameters selected after cross-validation:

Table 90: Optimal Parameters for the Elastic Net algorithm

\begin{tabular}{|c|c|}
\hline alpha & lambda \\
\hline 0.1642857 & 0.0052521 \\
\hline
\end{tabular}

Table 91: Optimal Parameters for the Random Forest algorithm

\begin{tabular}{|c|}
\hline mtry \\
\hline 2 \\
\hline
\end{tabular}

Table 92: Optimal Parameters for the XGB algorithm

\begin{tabular}{|c|c|c|c|c|c|c|}
\hline nrounds & max_depth & eta & gamma & colsample_bytree & min_child_weight & subsample \\
\hline 50 & 1 & 0.3 & 0 & 0.6 & 1 & 0.5 \\
\hline
\end{tabular}

Table 93: Optimal Parameters for the KRLS algorithm

\begin{tabular}{|c|c|c|c|}
\hline loss & epsilon & $\mathbf{b}$ & lambda \\
\hline logistic & 0.005 & 52 & $6.05 \mathrm{e}-05$ \\
\hline
\end{tabular}


medRxiv preprint doi: https://doi.org/10.1101/2021.07.20.21260818; this version posted July 23, 2021. The copyright holder for this preprint (which was not certified by peer review) is the author/funder, who has granted medRxiv a license to display the preprint in perpetuity.

\section{B.12.4 Table of Results}

Table 94: Manual Cross-Validation Results

\begin{tabular}{|c|c|c|c|c|c|c|c|c|c|}
\hline Algorithm & Recall 10 & Recall 20 & ROC & AUC & MRD & MRR & MSE & F1 & Efficiency Gain \\
\hline Ensemble & 0.211 & 0.350 & 0.619 & 0.097 & 0.009 & 1.238 & 0.040 & 0.933 & 1.937 \\
\hline KRLS & 0.202 & 0.321 & 0.594 & 0.071 & 0.006 & 1.153 & 0.041 & 0.933 & 1.857 \\
\hline Elastic Net & 0.164 & 0.301 & 0.582 & 0.061 & 0.004 & 1.086 & 0.041 & 0.931 & 1.508 \\
\hline Random Forest & 0.204 & 0.310 & 0.600 & 0.115 & 0.017 & 1.910 & 0.040 & 0.933 & 1.878 \\
\hline Wealth & 0.109 & 0.215 & 0.533 & 0.047 & 0.063 & 1.456 & 0.824 & 0.929 & 1.000 \\
\hline XGB & 0.184 & 0.319 & 0.594 & 0.068 & 0.007 & 1.175 & 0.041 & 0.932 & 1.692 \\
\hline
\end{tabular}

Table 95: Distribution of individuals in the top risk decile of each algorithm among each wealth decile

\begin{tabular}{l|r|r|r|r|c}
\hline Wealth Decile & Elastic Net & Ensemble & KRLS & Random Forest & XGB \\
\hline 1 & 0.280 & 0.218 & 0.227 & 0.195 & 0.121 \\
\hline 2 & 0.150 & 0.142 & 0.129 & 0.104 & 0.152 \\
\hline 3 & 0.111 & 0.135 & 0.130 & 0.096 & 0.177 \\
\hline 4 & 0.114 & 0.129 & 0.128 & 0.108 & 0.151 \\
\hline 5 & 0.070 & 0.073 & 0.092 & 0.059 & 0.079 \\
\hline 6 & 0.089 & 0.062 & 0.091 & 0.064 & 0.086 \\
\hline 7 & 0.060 & 0.061 & 0.060 & 0.063 & 0.062 \\
\hline 8 & 0.050 & 0.064 & 0.069 & 0.082 & 0.062 \\
\hline 9 & 0.042 & 0.056 & 0.042 & 0.092 & 0.050 \\
\hline 10 & 0.033 & 0.066 & 0.030 & 0.135 & 0.057 \\
\hline
\end{tabular}

\section{B.12.5 Performance Plots}

Below are various plots showing the performance for each model considered in Malawi. 
medRxiv preprint doi: https://doi.org/10.1101/2021.07.20.21260818; this version posted July 23, 2021. The copyright holder for this preprint (which was not certified by peer review) is the author/funder, who has granted medRxiv a license to display the preprint in perpetuity. All rights reserved. No reuse allowed without permission.
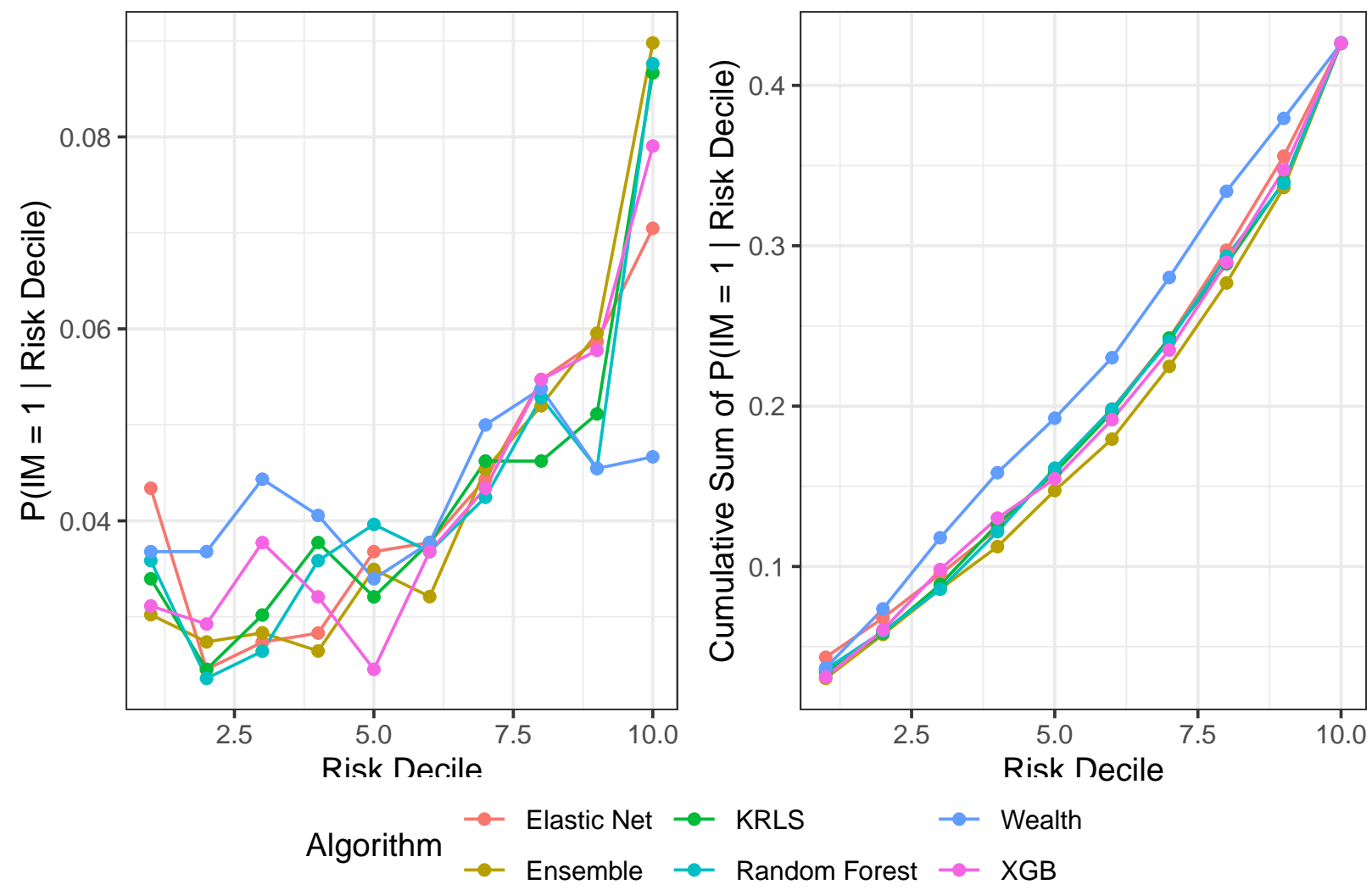

Figure 31: Probability of a mortality given that the observation is in a particular risk decile 
medRxiv preprint doi: https://doi.org/10.1101/2021.07.20.21260818; this version posted July 23, 2021. The copyright holder for this preprint (which was not certified by peer review) is the author/funder, who has granted medRxiv a license to display the preprint in perpetuity. All rights reserved. No reuse allowed without permission.

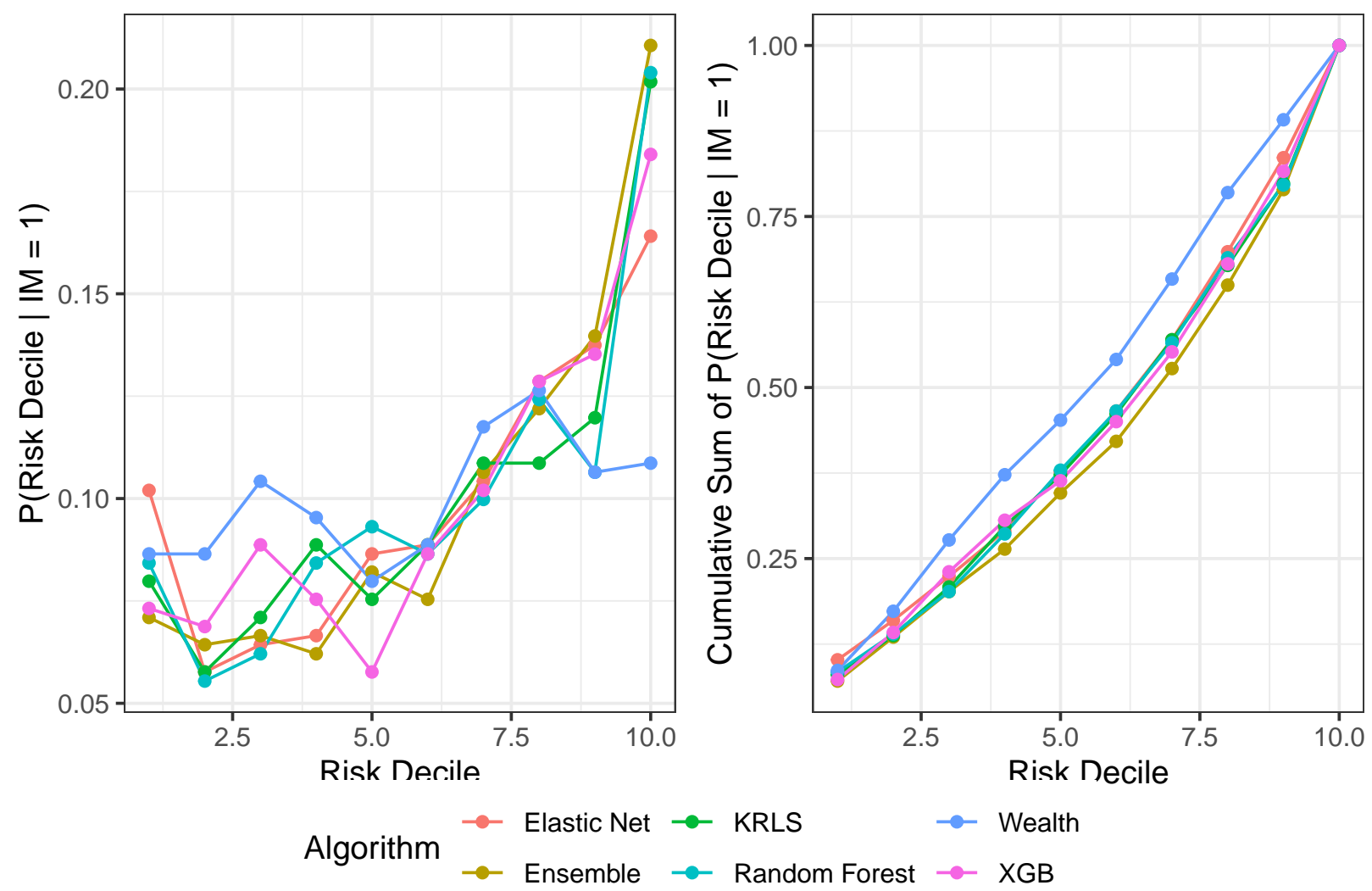

Figure 32: Probability of membership in a particular risk decile given a mortality 


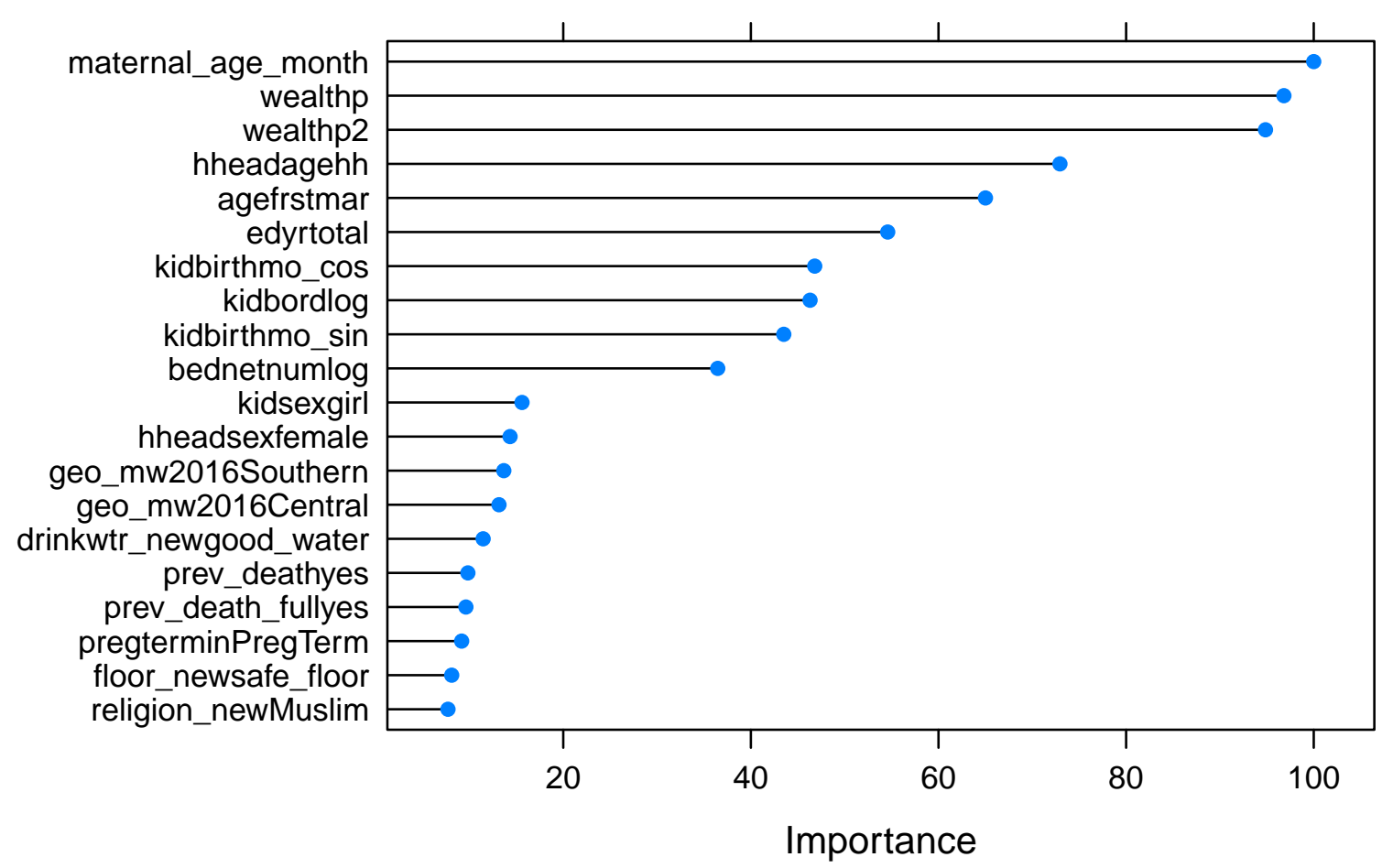

Figure 33: Variable Importance Plot generated by the Random Forest model

\section{B.12.6 Variable Importance Plot}

In this section we will show some measures to give some indication of the important variables necessary for predicting infant mortality. 
medRxiv preprint doi: https://doi.org/10.1101/2021.07.20.21260818; this version posted July 23, 2021. The copyright holder for this preprint (which was not certified by peer review) is the author/funder, who has granted medRxiv a license to display the preprint in perpetuity.

All rights reserved. No reuse allowed without permission.

\section{B.12.7 Elastic Net Coefficients}

Table 96: Standardized coefficients from the optimal elastic net model after 10-fold crossvalidation.

\begin{tabular}{|c|c|c|}
\hline & Coefficients & Standardized Coefficients \\
\hline (Intercept) & -2.8538 & -2.8538 \\
\hline pregterminPregTerm & -0.0339 & -0.0103 \\
\hline agefrstmar & 0.0058 & 0.0194 \\
\hline hheadagehh & $-8 \mathrm{e}-04$ & -0.0088 \\
\hline urbanRural & 0.0638 & 0.023 \\
\hline kidsexgirl & -0.1948 & -0.0974 \\
\hline hheadsexfemale & 0.0853 & 0.0363 \\
\hline edyrtotal & -0.0183 & -0.0653 \\
\hline drinkwtr_newgood_water & 0.0281 & 0.0109 \\
\hline floor_newsafe_floor & $-2 \mathrm{e}-04$ & $-1 e-04$ \\
\hline maternal_age_month & - & - \\
\hline cookfuel_newclean_fuel & - & - \\
\hline toilettype_newpit & -0.2427 & -0.0694 \\
\hline toilettype_newunimproved & 0.2467 & 0.0623 \\
\hline religion_newChristian & 0.0899 & 0.0311 \\
\hline religion_newHindu & - & - \\
\hline religion_newMuslim & - & - \\
\hline religion_newOther & -0.4883 & -0.0393 \\
\hline bednetnumlog & - & - \\
\hline prev_deathyes & 0.3763 & 0.1294 \\
\hline prev_death_fullyes & 0.2103 & 0.0827 \\
\hline kidbordlog & -0.2498 & -0.1686 \\
\hline kidbirthmo_sin & -0.0014 & -0.001 \\
\hline kidbirthmo_cos & 0.0452 & 0.032 \\
\hline geo_mw2016Central & 0.0838 & 0.04 \\
\hline geo_mw2016Southern & - & - \\
\hline wealthp & - & - \\
\hline wealthp2 & -0.0035 & -0.001 \\
\hline
\end{tabular}


medRxiv preprint doi: https://doi.org/10.1101/2021.07.20.21260818; this version posted July 23, 2021. The copyright holder for this preprint (which was not certified by peer review) is the author/funder, who has granted medRxiv a license to display the preprint in perpetuity.

All rights reserved. No reuse allowed without permission.

\section{B.13 Mali}

This sample was taken in the year 2012. There are 7913 observations in the dataset, and 6331 observations were used for the training set. Also, 130 observations were removed due to missing values. Finally, there were 475 deaths for the full dataset, and 380 deaths in the training set. 


\section{B.13.1 Variables Used}

Table 97: Variables included in the model for Mali

\begin{tabular}{|c|c|}
\hline Variable & Type \\
\hline mortality.under $12 \mathrm{~m}$ & categorical \\
\hline pregtermin & categorical \\
\hline agefrstmar & numeric \\
\hline hheadagehh & numeric \\
\hline urban & categorical \\
\hline kidsex & categorical \\
\hline hheadsex & categorical \\
\hline edyrtotal & numeric \\
\hline drinkwtr_new & categorical \\
\hline floor_new & categorical \\
\hline maternal_age_month & numeric \\
\hline toilettype_new & categorical \\
\hline religion_new & categorical \\
\hline bednetnumlog & numeric \\
\hline prev_death & categorical \\
\hline prev_death_full & categorical \\
\hline kidbordlog & numeric \\
\hline kidbirthmo_sin & numeric \\
\hline kidbirthmo_cos & numeric \\
\hline geo_ml2012 & categorical \\
\hline wealthp & numeric \\
\hline wealthp2 & numeric \\
\hline
\end{tabular}




\section{B.13.2 Mortality Breakdown by Wealth Quintile}

\section{B.13.3 Optimal Parameters}

These are the algorithm parameters selected after cross-validation:

Table 98: Optimal Parameters for the Elastic Net algorithm

\begin{tabular}{|c|c|}
\hline alpha & lambda \\
\hline 0.1 & 0.0091994 \\
\hline
\end{tabular}

Table 99: Optimal Parameters for the Random Forest algorithm

\begin{tabular}{|c|}
\hline mtry \\
\hline 8 \\
\hline
\end{tabular}

Table 100: Optimal Parameters for the XGB algorithm

\begin{tabular}{|c|c|c|c|c|c|c|}
\hline nrounds & max_depth & eta & gamma & colsample_bytree & min_child_weight & subsample \\
\hline 50 & 1 & 0.3 & 0 & 0.6 & 1 & 1 \\
\hline
\end{tabular}

Table 101: Optimal Parameters for the KRLS algorithm

\begin{tabular}{|c|c|c|c|}
\hline loss & epsilon & $\mathbf{b}$ & lambda \\
\hline logistic & 0.005 & 56 & 0.0001062 \\
\hline
\end{tabular}


medRxiv preprint doi: https://doi.org/10.1101/2021.07.20.21260818; this version posted July 23, 2021. The copyright holder for this preprint (which was not certified by peer review) is the author/funder, who has granted medRxiv a license to display the preprint in perpetuity.

\section{B.13.4 Table of Results}

Table 102: Manual Cross-Validation Results

\begin{tabular}{|c|c|c|c|c|c|c|c|c|c|}
\hline Algorithm & Recall 10 & Recall 20 & ROC & AUC & MRD & MRR & MSE & F1 & Efficiency Gain \\
\hline Ensemble & 0.245 & 0.371 & 0.666 & 0.147 & 0.025 & 1.410 & 0.055 & 0.929 & 2.114 \\
\hline KRLS & 0.213 & 0.379 & 0.657 & 0.110 & 0.018 & 1.299 & 0.055 & 0.927 & 1.841 \\
\hline Elastic Net & 0.203 & 0.355 & 0.646 & 0.105 & 0.015 & 1.262 & 0.056 & 0.927 & 1.750 \\
\hline Random Forest & 0.221 & 0.347 & 0.634 & 0.142 & 0.050 & 1.742 & 0.057 & 0.928 & 1.909 \\
\hline Wealth & 0.116 & 0.216 & 0.555 & 0.068 & 0.181 & 3.474 & 0.833 & 0.921 & 1.000 \\
\hline XGB & 0.208 & 0.358 & 0.654 & 0.112 & 0.017 & 1.291 & 0.055 & 0.927 & 1.795 \\
\hline
\end{tabular}

Table 103: Distribution of individuals in the top risk decile of each algorithm among each wealth decile

\begin{tabular}{l|r|r|r|r|r}
\hline Wealth Decile & Elastic Net & Ensemble & KRLS & Random Forest & XGB \\
\hline 1 & 0.119 & 0.132 & 0.111 & 0.148 & 0.108 \\
\hline 2 & 0.136 & 0.109 & 0.125 & 0.104 & 0.111 \\
\hline 3 & 0.111 & 0.081 & 0.090 & 0.067 & 0.100 \\
\hline 4 & 0.124 & 0.146 & 0.138 & 0.132 & 0.154 \\
\hline 5 & 0.130 & 0.148 & 0.146 & 0.133 & 0.156 \\
\hline 6 & 0.152 & 0.171 & 0.154 & 0.151 & 0.187 \\
\hline 7 & 0.114 & 0.122 & 0.118 & 0.112 & 0.141 \\
\hline 8 & 0.059 & 0.048 & 0.067 & 0.049 & 0.019 \\
\hline 9 & 0.044 & 0.030 & 0.041 & 0.038 & 0.017 \\
\hline 10 & 0.010 & 0.017 & 0.008 & 0.065 & 0.006 \\
\hline
\end{tabular}

\section{B.13.5 Performance Plots}

Below are various plots showing the performance for each model considered in Mali. 
medRxiv preprint doi: https://doi.org/10.1101/2021.07.20.21260818; this version posted July 23, 2021. The copyright holder for this preprint (which was not certified by peer review) is the author/funder, who has granted medRxiv a license to display the preprint in perpetuity. All rights reserved. No reuse allowed without permission.

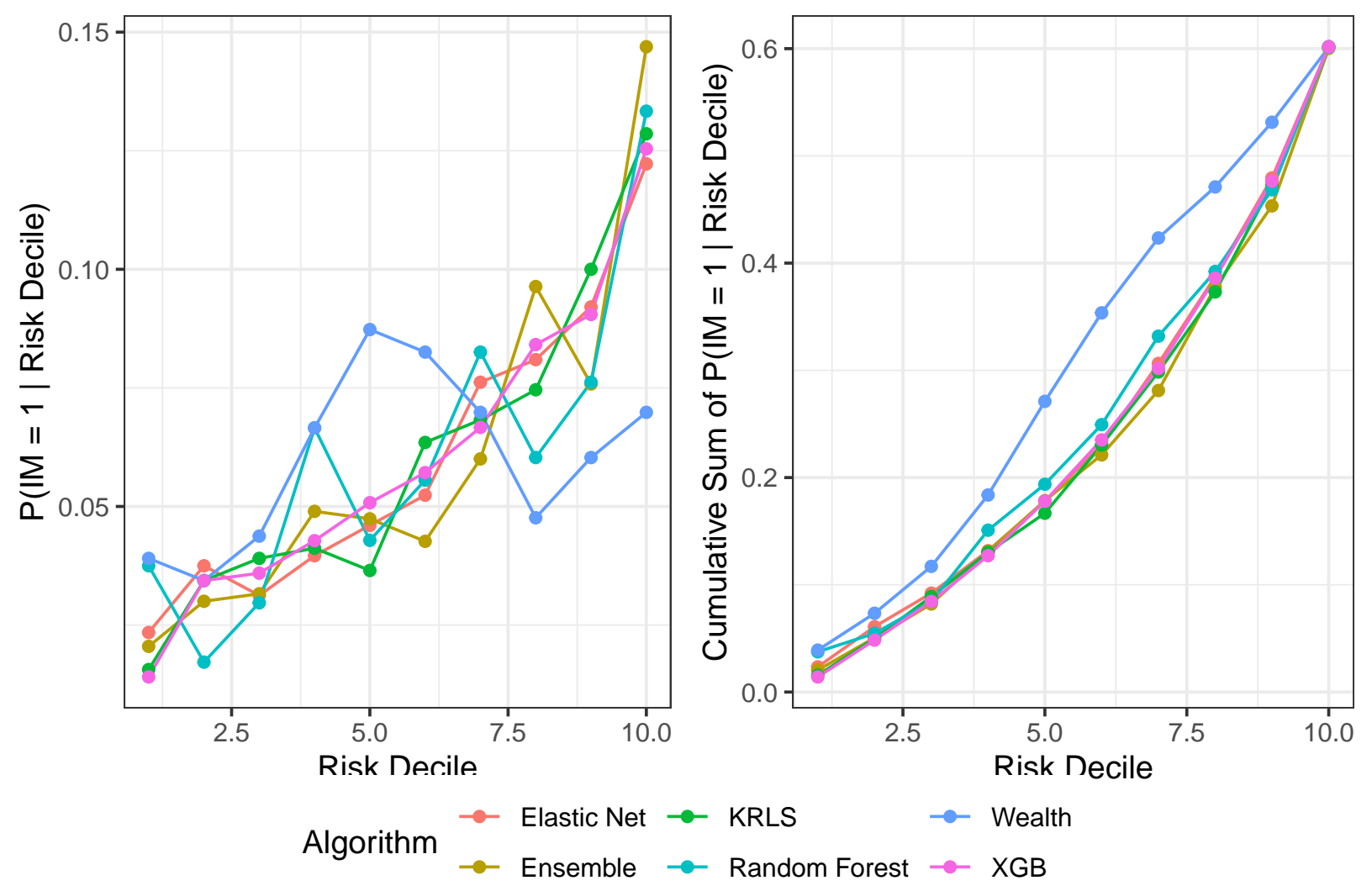

Figure 34: Probability of a mortality given that the observation is in a particular risk decile 
medRxiv preprint doi: https://doi.org/10.1101/2021.07.20.21260818; this version posted July 23, 2021. The copyright holder for this preprint (which was not certified by peer review) is the author/funder, who has granted medRxiv a license to display the preprint in perpetuity. All rights reserved. No reuse allowed without permission.
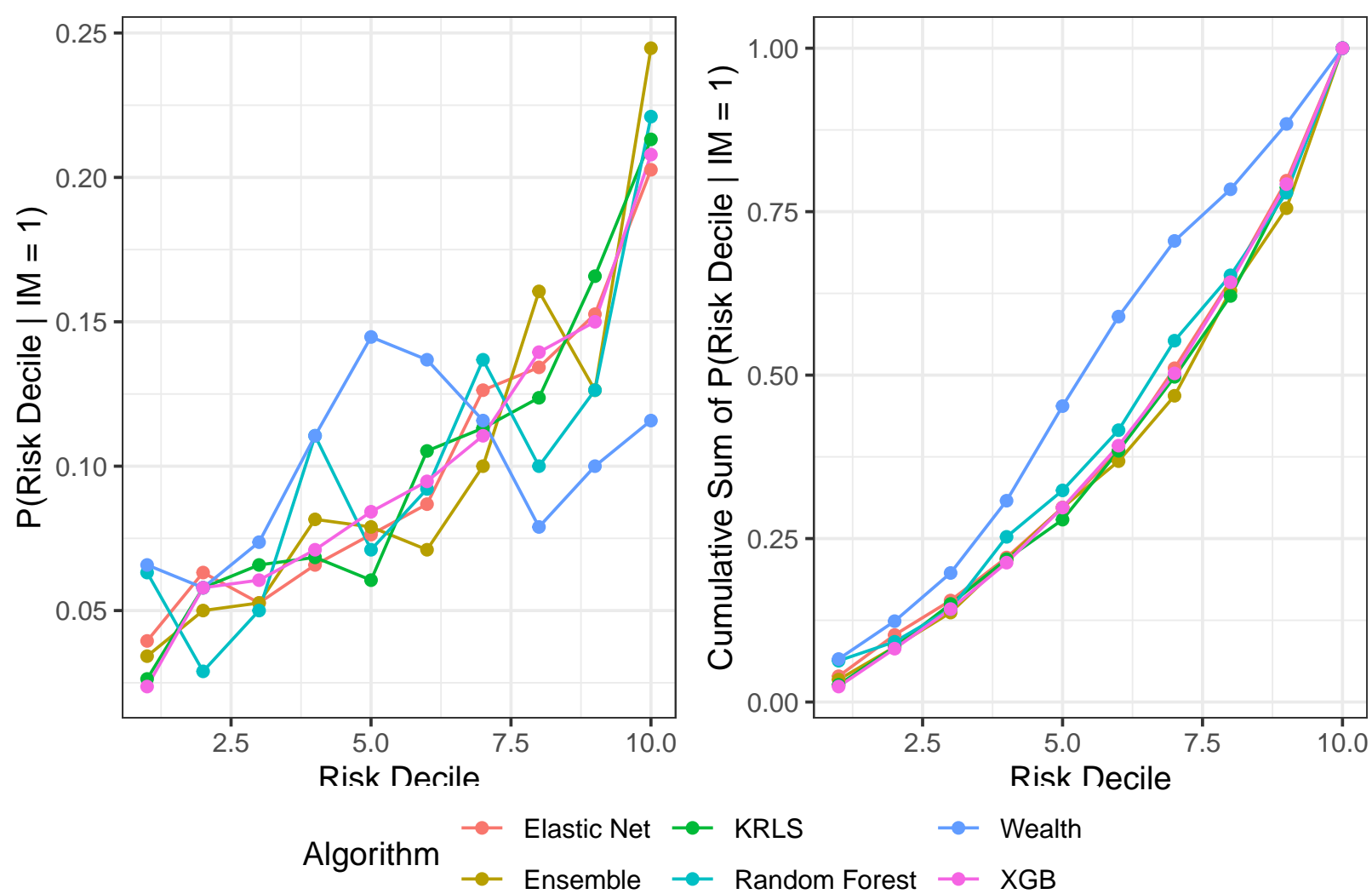

Figure 35: Probability of membership in a particular risk decile given a mortality 


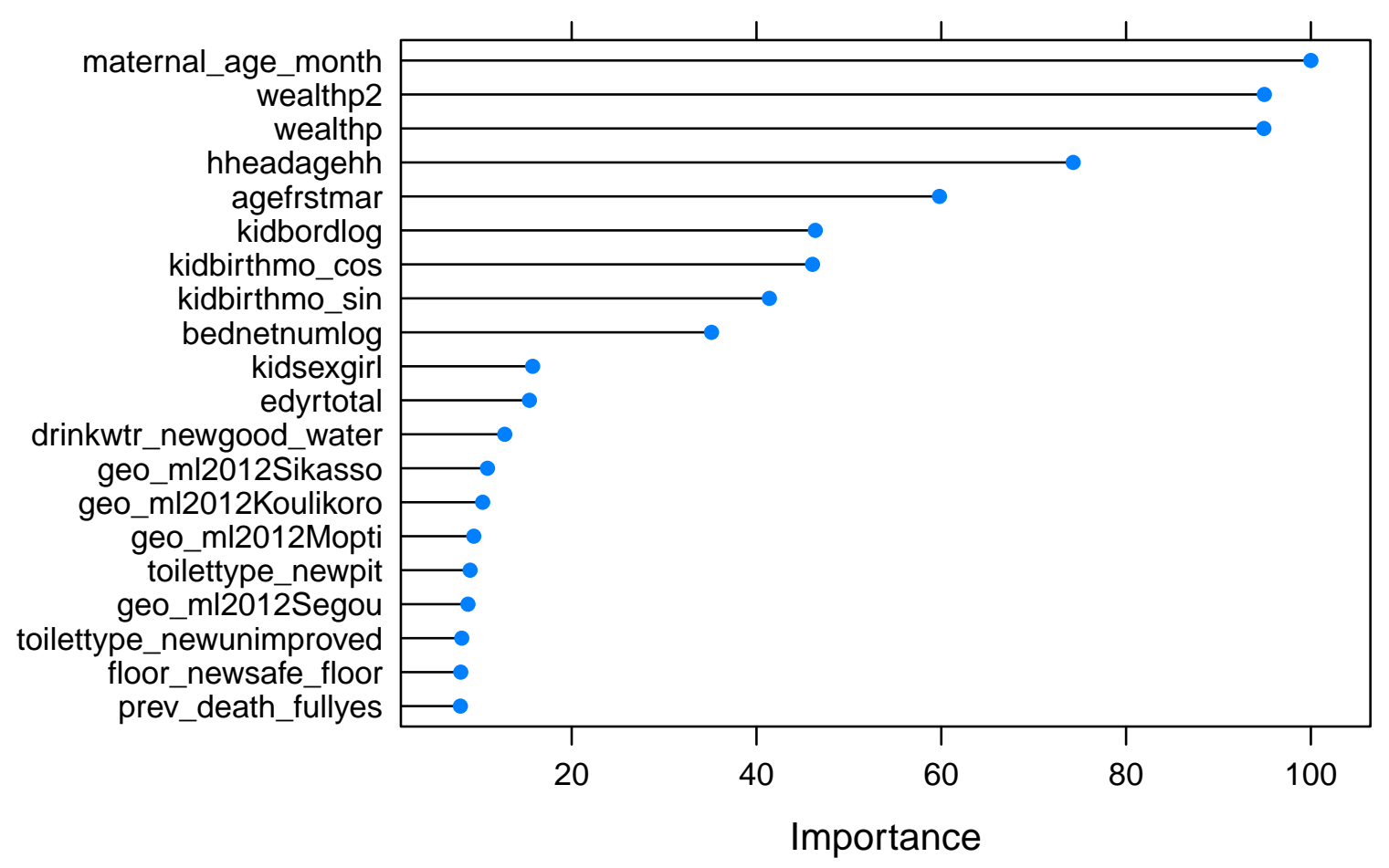

Figure 36: Variable Importance Plot generated by the Random Forest model

\section{B.13.6 Variable Importance Plot}

In this section we will show some measures to give some indication of the important variables necessary for predicting infant mortality. 
medRxiv preprint doi: https://doi.org/10.1101/2021.07.20.21260818; this version posted July 23, 2021. The copyright holder for this preprint (which was not certified by peer review) is the author/funder, who has granted medRxiv a license to display the preprint in perpetuity.

All rights reserved. No reuse allowed without permission.

\section{B.13.7 Elastic Net Coefficients}

Table 104: Standardized coefficients from the optimal elastic net model after 10-fold crossvalidation.

\begin{tabular}{|c|c|c|}
\hline & Coefficients & Standardized Coefficients \\
\hline (Intercept) & -2.3406 & -2.3406 \\
\hline pregterminPregTerm & -0.1313 & -0.0349 \\
\hline agefrstmar & -0.0104 & -0.0414 \\
\hline hheadagehh & 0.0012 & 0.0141 \\
\hline urbanRural & 0.3267 & 0.1378 \\
\hline kidsexgirl & -0.2961 & -0.148 \\
\hline hheadsexfemale & 0.2519 & 0.0621 \\
\hline edyrtotal & -0.0201 & -0.0588 \\
\hline drinkwtr_newgood_water & 0.036 & 0.0171 \\
\hline floor_newsafe_floor & 0.0548 & 0.0242 \\
\hline maternal_age_month & $-8 \mathrm{e}-04$ & -0.0667 \\
\hline toilettype_newpit & - & - \\
\hline toilettype_newunimproved & 0.0054 & 0.0018 \\
\hline religion_newChristian & - & - \\
\hline religion_newHindu & - & - \\
\hline religion_newMuslim & -0.0948 & -0.0237 \\
\hline religion_newOther & 0.3268 & 0.0577 \\
\hline bednetnumlog & -0.0959 & -0.0472 \\
\hline prev_deathyes & 0.2885 & 0.1097 \\
\hline prev_death_fullyes & 0.722 & 0.3026 \\
\hline kidbordlog & -0.205 & -0.1388 \\
\hline kidbirthmo_sin & 0.0559 & 0.0403 \\
\hline kidbirthmo_cos & -0.0222 & -0.0151 \\
\hline geo_ml2012Koulikoro & - & - \\
\hline geo_ml2012Sikasso & 0.1817 & 0.0699 \\
\hline geo_ml2012Segou & -0.1112 & -0.0435 \\
\hline geo_ml2012Mopti & 0.0394 & 0.0144 \\
\hline geo_ml2012Bamako & -0.0041 & -0.0014 \\
\hline wealthp & - & - \\
\hline wealthp2 & -0.1554 & -0.0463 \\
\hline
\end{tabular}


medRxiv preprint doi: https://doi.org/10.1101/2021.07.20.21260818; this version posted July 23, 2021. The copyright holder for this preprint (which was not certified by peer review) is the author/funder, who has granted medRxiv a license to display the preprint in perpetuity.

All rights reserved. No reuse allowed without permission.

\section{B.14 Mozambique}

This sample was taken in the year 2011. There are 8026 observations in the dataset, and 6422 observations were used for the training set. Also, 467 observations were removed due to missing values. Finally, there were 537 deaths for the full dataset, and 430 deaths in the training set. 
medRxiv preprint doi: https://doi.org/10.1101/2021.07.20.21260818; this version posted July 23, 2021. The copyright holder for this preprint (which was not certified by peer review) is the author/funder, who has granted medRxiv a license to display the preprint in perpetuity.

All rights reserved. No reuse allowed without permission. 


\section{B.14.1 Variables Used}

Table 105: Variables included in the model for Mozambique

\begin{tabular}{|c|c|}
\hline Variable & Туре \\
\hline mortality.under $12 \mathrm{~m}$ & categorical \\
\hline pregtermin & categorical \\
\hline agefrstmar & numeric \\
\hline hheadagehh & numeric \\
\hline urban & categorical \\
\hline kidsex & categorical \\
\hline hheadsex & categorical \\
\hline edyrtotal & numeric \\
\hline drinkwtr_new & categorical \\
\hline floor_new & categorical \\
\hline maternal_age_month & numeric \\
\hline cookfuel_new & categorical \\
\hline toilettype_new & categorical \\
\hline religion_new & categorical \\
\hline bednetnumlog & numeric \\
\hline prev_death & categorical \\
\hline prev_death_full & categorical \\
\hline malaria_new & numeric \\
\hline kidbordlog & numeric \\
\hline kidbirthmo_sin & numeric \\
\hline kidbirthmo_cos & numeric \\
\hline geo_mz2011 & categorical \\
\hline wealthp & numeric \\
\hline wealthp2 139 & numeric \\
\hline
\end{tabular}




\section{B.14.2 Mortality Breakdown by Wealth Quintile}

\section{B.14.3 Optimal Parameters}

These are the algorithm parameters selected after cross-validation:

Table 106: Optimal Parameters for the Elastic Net algorithm

\begin{tabular}{|c|c|}
\hline alpha & lambda \\
\hline 0.1 & 0.0147651 \\
\hline
\end{tabular}

Table 107: Optimal Parameters for the Random Forest algorithm

\begin{tabular}{|c|}
\hline mtry \\
\hline 10 \\
\hline
\end{tabular}

Table 108: Optimal Parameters for the XGB algorithm

\begin{tabular}{|c|c|c|c|c|c|c|}
\hline nrounds & max_depth & eta & gamma & colsample_bytree & min_child_weight & subsample \\
\hline 50 & 2 & 0.3 & 0 & 0.8 & 1 & 1 \\
\hline
\end{tabular}

Table 109: Optimal Parameters for the KRLS algorithm

\begin{tabular}{|c|c|c|c|}
\hline loss & epsilon & $\mathbf{b}$ & lambda \\
\hline logistic & 0.005 & 70 & 0.0001355 \\
\hline
\end{tabular}


medRxiv preprint doi: https://doi.org/10.1101/2021.07.20.21260818; this version posted July 23, 2021. The copyright holder for this preprint (which was not certified by peer review) is the author/funder, who has granted medRxiv a license to display the preprint in perpetuity.

\section{B.14.4 Table of Results}

Table 110: Manual Cross-Validation Results

\begin{tabular}{|c|c|c|c|c|c|c|c|c|c|}
\hline Algorithm & Recall 10 & Recall 20 & ROC & AUC & MRD & MRR & MSE & F1 & Efficiency Gain \\
\hline Ensemble & 0.240 & 0.370 & 0.631 & 0.150 & 0.020 & 1.296 & 0.061 & 0.926 & 2.341 \\
\hline KRLS & 0.207 & 0.323 & 0.591 & 0.111 & 0.010 & 1.148 & 0.062 & 0.924 & 2.023 \\
\hline Elastic Net & 0.156 & 0.286 & 0.567 & 0.097 & 0.005 & 1.077 & 0.062 & 0.921 & 1.523 \\
\hline Random Forest & 0.244 & 0.365 & 0.625 & 0.158 & 0.051 & 1.676 & 0.062 & 0.927 & 2.386 \\
\hline Wealth & 0.102 & 0.242 & 0.536 & 0.073 & 0.089 & 2.105 & 0.888 & 0.917 & 1.000 \\
\hline XGB & 0.179 & 0.326 & 0.598 & 0.110 & 0.015 & 1.228 & 0.062 & 0.922 & 1.750 \\
\hline
\end{tabular}

Table 111: Distribution of individuals in the top risk decile of each algorithm among each wealth decile

\begin{tabular}{l|r|r|r|r|r}
\hline Wealth Decile & Elastic Net & Ensemble & KRLS & Random Forest & XGB \\
\hline 1 & 0.233 & 0.184 & 0.228 & 0.159 & 0.148 \\
\hline 2 & 0.195 & 0.188 & 0.190 & 0.145 & 0.198 \\
\hline 3 & 0.159 & 0.108 & 0.150 & 0.081 & 0.123 \\
\hline 4 & 0.139 & 0.145 & 0.142 & 0.127 & 0.141 \\
\hline 5 & 0.092 & 0.106 & 0.109 & 0.095 & 0.125 \\
\hline 6 & 0.080 & 0.092 & 0.086 & 0.100 & 0.073 \\
\hline 7 & 0.053 & 0.048 & 0.033 & 0.055 & 0.040 \\
\hline 8 & 0.025 & 0.062 & 0.030 & 0.098 & 0.059 \\
\hline 9 & 0.017 & 0.033 & 0.020 & 0.061 & 0.041 \\
\hline 10 & 0.006 & 0.034 & 0.011 & 0.078 & 0.050 \\
\hline
\end{tabular}

\section{B.14.5 Performance Plots}

Below are various plots showing the performance for each model considered in Mozambique. 
medRxiv preprint doi: https://doi.org/10.1101/2021.07.20.21260818; this version posted July 23, 2021. The copyright holder for this preprint (which was not certified by peer review) is the author/funder, who has granted medRxiv a license to display the preprint in perpetuity. All rights reserved. No reuse allowed without permission.

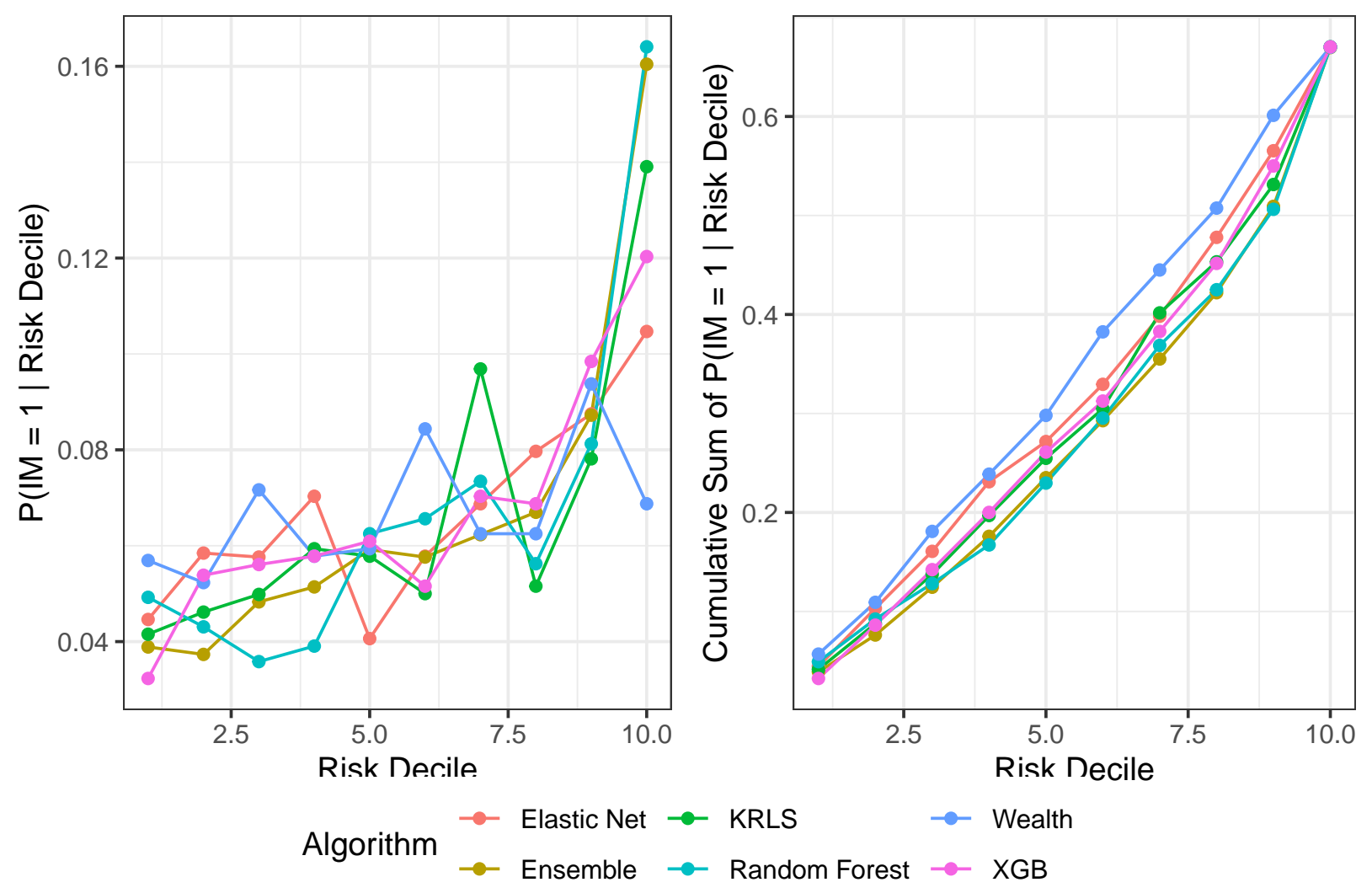

Figure 37: Probability of a mortality given that the observation is in a particular risk decile 
medRxiv preprint doi: https://doi.org/10.1101/2021.07.20.21260818; this version posted July 23, 2021. The copyright holder for this preprint (which was not certified by peer review) is the author/funder, who has granted medRxiv a license to display the preprint in perpetuity. All rights reserved. No reuse allowed without permission.
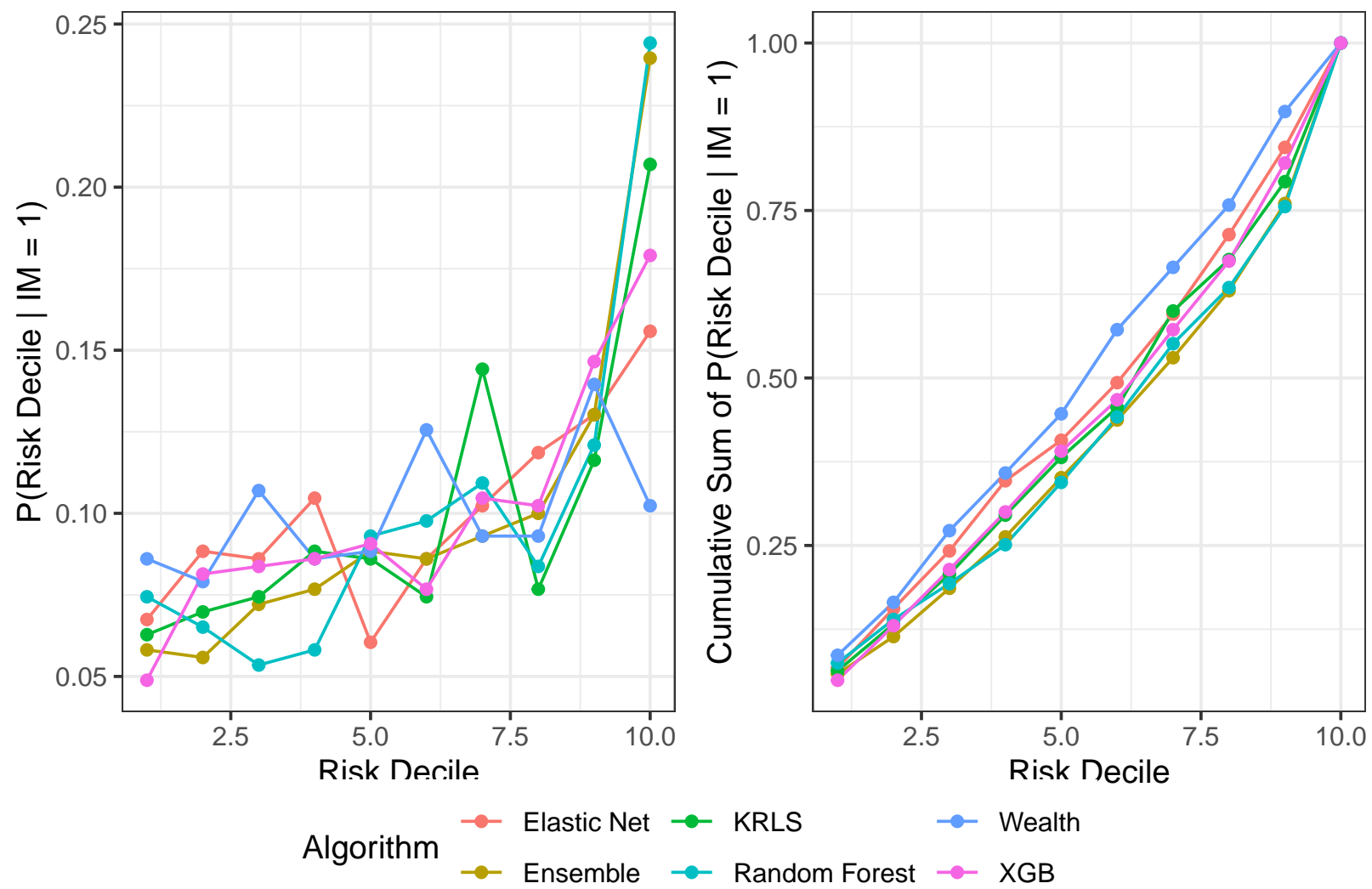

Figure 38: Probability of membership in a particular risk decile given a mortality 


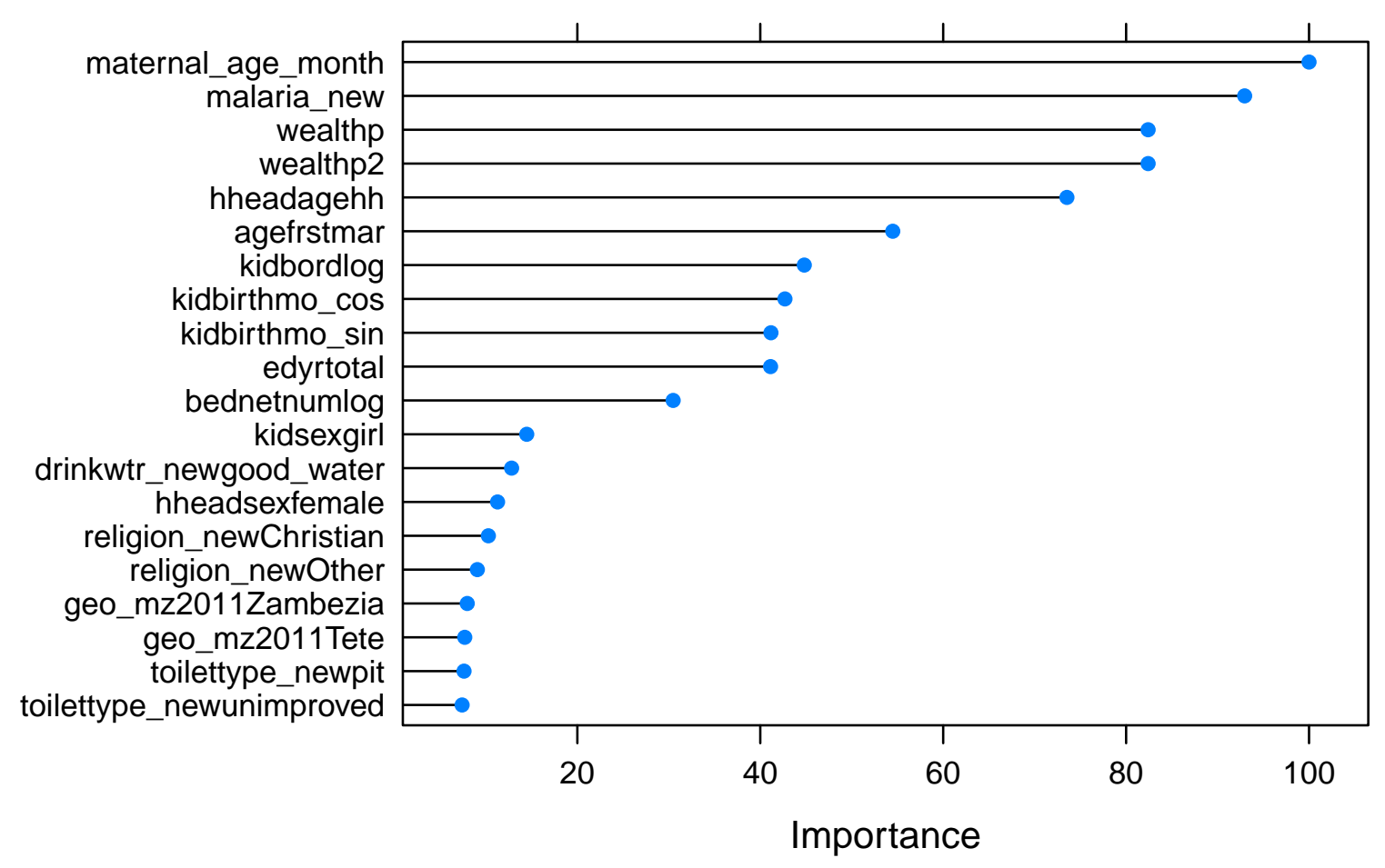

Figure 39: Variable Importance Plot generated by the Random Forest model

\section{B.14.6 Variable Importance Plot}

In this section we will show some measures to give some indication of the important variables necessary for predicting infant mortality. 
medRxiv preprint doi: https://doi.org/10.1101/2021.07.20.21260818; this version posted July 23, 2021. The copyright holder for this preprint (which was not certified by peer review) is the author/funder, who has granted medRxiv a license to display the preprint in perpetuity.

All rights reserved. No reuse allowed without permission.

\section{B.14.7 Elastic Net Coefficients}

Table 112: Standardized coefficients from the optimal elastic net model after 10-fold crossvalidation.

\begin{tabular}{|c|c|c|}
\hline & Coefficients & Standardized Coefficients \\
\hline (Intercept) & -2.5583 & -2.5583 \\
\hline pregterminPregTerm & - & - \\
\hline agefrstmar & - & - \\
\hline hheadagehh & -0.0016 & -0.0198 \\
\hline urbanRural & 0.1086 & 0.0501 \\
\hline kidsexgirl & -0.0489 & -0.0244 \\
\hline hheadsexfemale & 0.0672 & 0.031 \\
\hline edyrtotal & - & - \\
\hline drinkwtr_newgood_water & - & - \\
\hline floor_newsafe_floor & - & - \\
\hline maternal_age_month & -0.001 & -0.085 \\
\hline cookfuel_newclean_fuel & -0.1191 & -0.0196 \\
\hline toilettype_newpit & - & - \\
\hline toilettype_newunimproved & - & - \\
\hline religion_newChristian & - & - \\
\hline religion_newHindu & - & - \\
\hline religion_newMuslim & - & - \\
\hline religion_newOther & 0.0117 & 0.0056 \\
\hline bednetnumlog & - & - \\
\hline prev_deathyes & 0.291 & 0.1152 \\
\hline prev_death_fullyes & 0.1114 & 0.048 \\
\hline malaria_new & 0.3039 & 0.0472 \\
\hline kidbordlog & -0.0452 & -0.0308 \\
\hline kidbirthmo_sin & 0.1028 & 0.0734 \\
\hline kidbirthmo_cos & - & - \\
\hline geo_mz2011Cabo delgado & -0.2012 & -0.0578 \\
\hline geo_mz2011Nampula & -0.1401 & -0.0389 \\
\hline geo_mz2011Zambezia & 0.2932 & 0.098 \\
\hline geo_mz2011Tete & 0.3138 & 0.0934 \\
\hline geo_mz2011Manica & - & - \\
\hline geo_mz2011Sofala & - & - \\
\hline geo_mz2011Inhambane & -0.1593 & -0.0411 \\
\hline geo_mz2011Gaza & -0.0092 & -0.0025 \\
\hline geo_mz2011Maputo Provincia & 0.0655 & 0.0168 \\
\hline geo_mz2011City of Maputo & 0.0165 & 0.0041 \\
\hline wealthp & - & - \\
\hline wealthp2 & - & - \\
\hline
\end{tabular}


medRxiv preprint doi: https://doi.org/10.1101/2021.07.20.21260818; this version posted July 23, 2021. The copyright holder for this preprint (which was not certified by peer review) is the author/funder, who has granted medRxiv a license to display the preprint in perpetuity.

All rights reserved. No reuse allowed without permission.

\section{B.15 Niger}

This sample was taken in the year 2012. There are 9612 observations in the dataset, and 7690 observations were used for the training set. Also, 38 observations were removed due to missing values. Finally, there were 586 deaths for the full dataset, and 469 deaths in the training set. 


\section{B.15.1 Variables Used}

Table 113: Variables included in the model for Niger

\begin{tabular}{|c|c|}
\hline Variable & Type \\
\hline mortality.under $12 \mathrm{~m}$ & categorical \\
\hline pregtermin & categorical \\
\hline agefrstmar & numeric \\
\hline hheadagehh & numeric \\
\hline urban & categorical \\
\hline kidsex & categorical \\
\hline hheadsex & categorical \\
\hline edyrtotal & numeric \\
\hline drinkwtr_new & categorical \\
\hline floor_new & categorical \\
\hline maternal_age_month & numeric \\
\hline cookfuel_new & categorical \\
\hline toilettype_new & categorical \\
\hline bednetnumlog & numeric \\
\hline prev_death & categorical \\
\hline prev_death_full & categorical \\
\hline kidbordlog & numeric \\
\hline kidbirthmo_sin & numeric \\
\hline kidbirthmo_cos & numeric \\
\hline geo_ne2012 & categorical \\
\hline wealthp & numeric \\
\hline wealthp2 & numeric \\
\hline
\end{tabular}




\section{B.15.2 Mortality Breakdown by Wealth Quintile}

\section{B.15.3 Optimal Parameters}

These are the algorithm parameters selected after cross-validation:

Table 114: Optimal Parameters for the Elastic Net algorithm

\begin{tabular}{|c|c|}
\hline alpha & lambda \\
\hline 0.1642857 & 0.0106825 \\
\hline
\end{tabular}

Table 115: Optimal Parameters for the Random Forest algorithm

\begin{tabular}{|c|}
\hline mtry \\
\hline 8 \\
\hline
\end{tabular}

Table 116: Optimal Parameters for the XGB algorithm

\begin{tabular}{|c|c|c|c|c|c|c|}
\hline nrounds & max_depth & eta & gamma & colsample_bytree & min_child_weight & subsample \\
\hline 50 & 1 & 0.3 & 0 & 0.6 & 1 & 1 \\
\hline
\end{tabular}

Table 117: Optimal Parameters for the KRLS algorithm

\begin{tabular}{|c|c|c|c|}
\hline loss & epsilon & $\mathbf{b}$ & lambda \\
\hline logistic & 0.005 & 58 & 0.0001792 \\
\hline
\end{tabular}


medRxiv preprint doi: https://doi.org/10.1101/2021.07.20.21260818; this version posted July 23, 2021. The copyright holder for this preprint (which was not certified by peer review) is the author/funder, who has granted medRxiv a license to display the preprint in perpetuity.

\section{B.15.4 Table of Results}

Table 118: Manual Cross-Validation Results

\begin{tabular}{|c|c|c|c|c|c|c|c|c|c|}
\hline Algorithm & Recall 10 & Recall 20 & ROC & AUC & MRD & MRR & MSE & F1 & Efficiency Gain \\
\hline Ensemble & 0.194 & 0.335 & 0.635 & 0.150 & 0.018 & 1.287 & 0.056 & 0.925 & 2.021 \\
\hline KRLS & 0.149 & 0.282 & 0.625 & 0.096 & 0.009 & 1.154 & 0.057 & 0.923 & 1.554 \\
\hline Elastic Net & 0.149 & 0.307 & 0.612 & 0.086 & 0.008 & 1.127 & 0.057 & 0.923 & 1.554 \\
\hline Random Forest & 0.205 & 0.307 & 0.610 & 0.144 & 0.046 & 1.671 & 0.057 & 0.927 & 2.131 \\
\hline Wealth & 0.096 & 0.196 & 0.532 & 0.067 & 0.158 & 2.954 & 0.802 & 0.919 & 1.000 \\
\hline XGB & 0.160 & 0.286 & 0.607 & 0.091 & 0.009 & 1.147 & 0.057 & 0.924 & 1.665 \\
\hline
\end{tabular}

Table 119: Distribution of individuals in the top risk decile of each algorithm among each wealth decile

\begin{tabular}{l|r|r|r|r|r}
\hline Wealth Decile & Elastic Net & Ensemble & KRLS & Random Forest & XGB \\
\hline 1 & 0.134 & 0.116 & 0.135 & 0.121 & 0.105 \\
\hline 2 & 0.178 & 0.118 & 0.159 & 0.091 & 0.138 \\
\hline 3 & 0.134 & 0.152 & 0.148 & 0.139 & 0.146 \\
\hline 4 & 0.120 & 0.141 & 0.126 & 0.112 & 0.134 \\
\hline 5 & 0.139 & 0.114 & 0.134 & 0.085 & 0.133 \\
\hline 6 & 0.124 & 0.135 & 0.126 & 0.114 & 0.138 \\
\hline 7 & 0.085 & 0.090 & 0.099 & 0.090 & 0.105 \\
\hline 8 & 0.066 & 0.093 & 0.059 & 0.112 & 0.074 \\
\hline 9 & 0.016 & 0.030 & 0.011 & 0.079 & 0.022 \\
\hline 10 & 0.003 & 0.018 & 0.000 & 0.055 & 0.003 \\
\hline
\end{tabular}

\section{B.15.5 Performance Plots}

Below are various plots showing the performance for each model considered in Niger. 


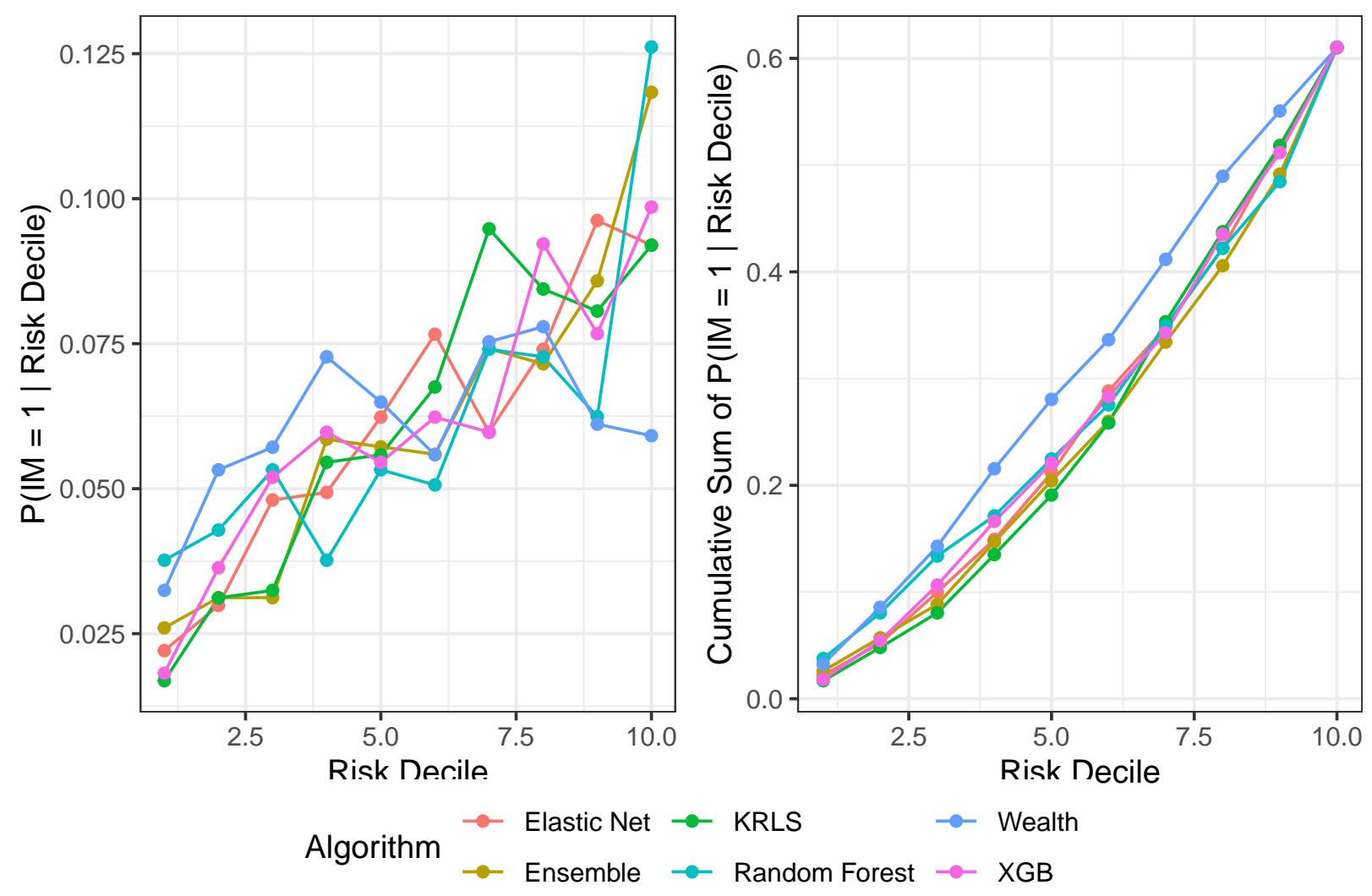

Figure 40: Probability of a mortality given that the observation is in a particular risk decile 

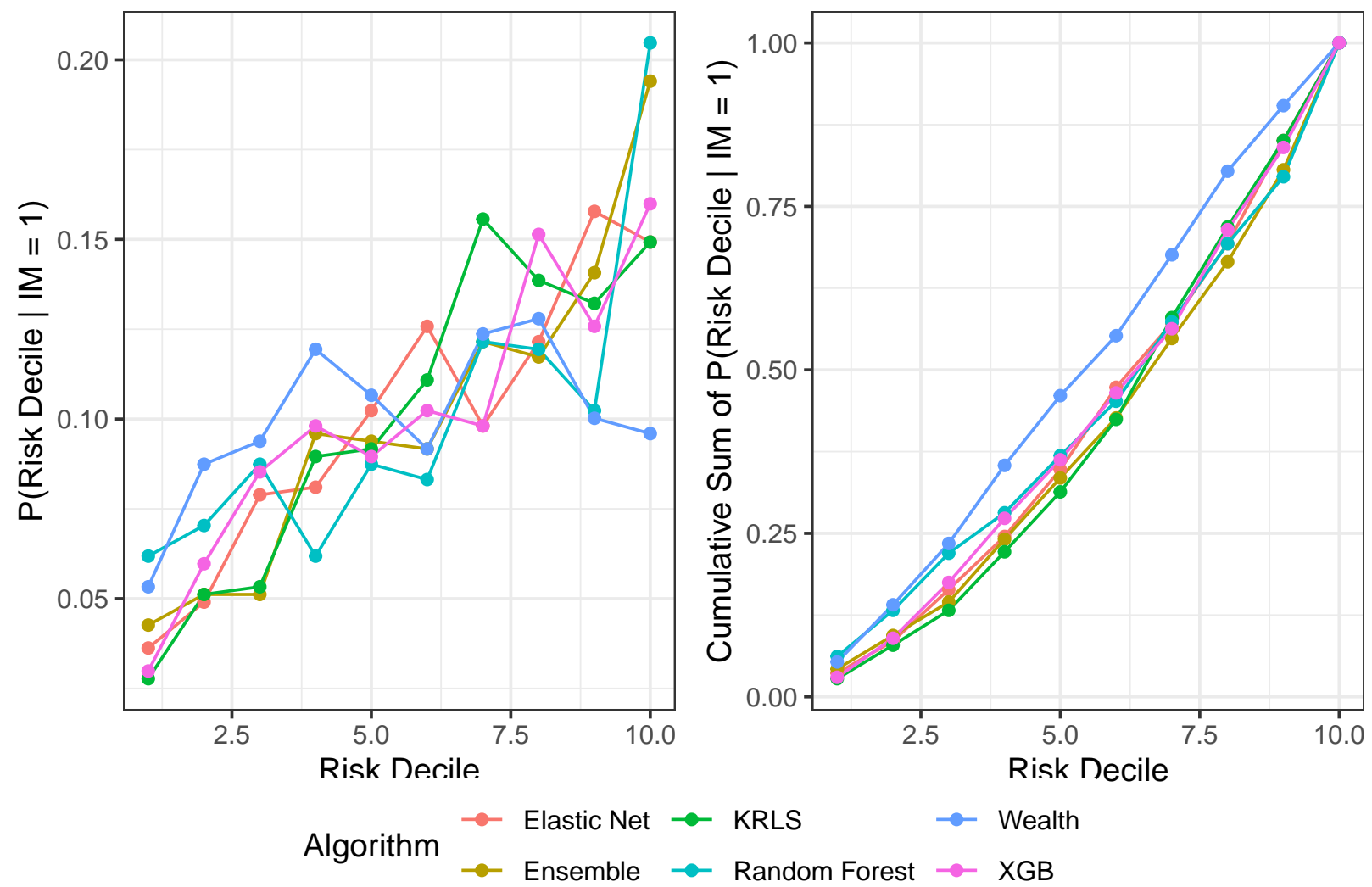

Figure 41: Probability of membership in a particular risk decile given a mortality 


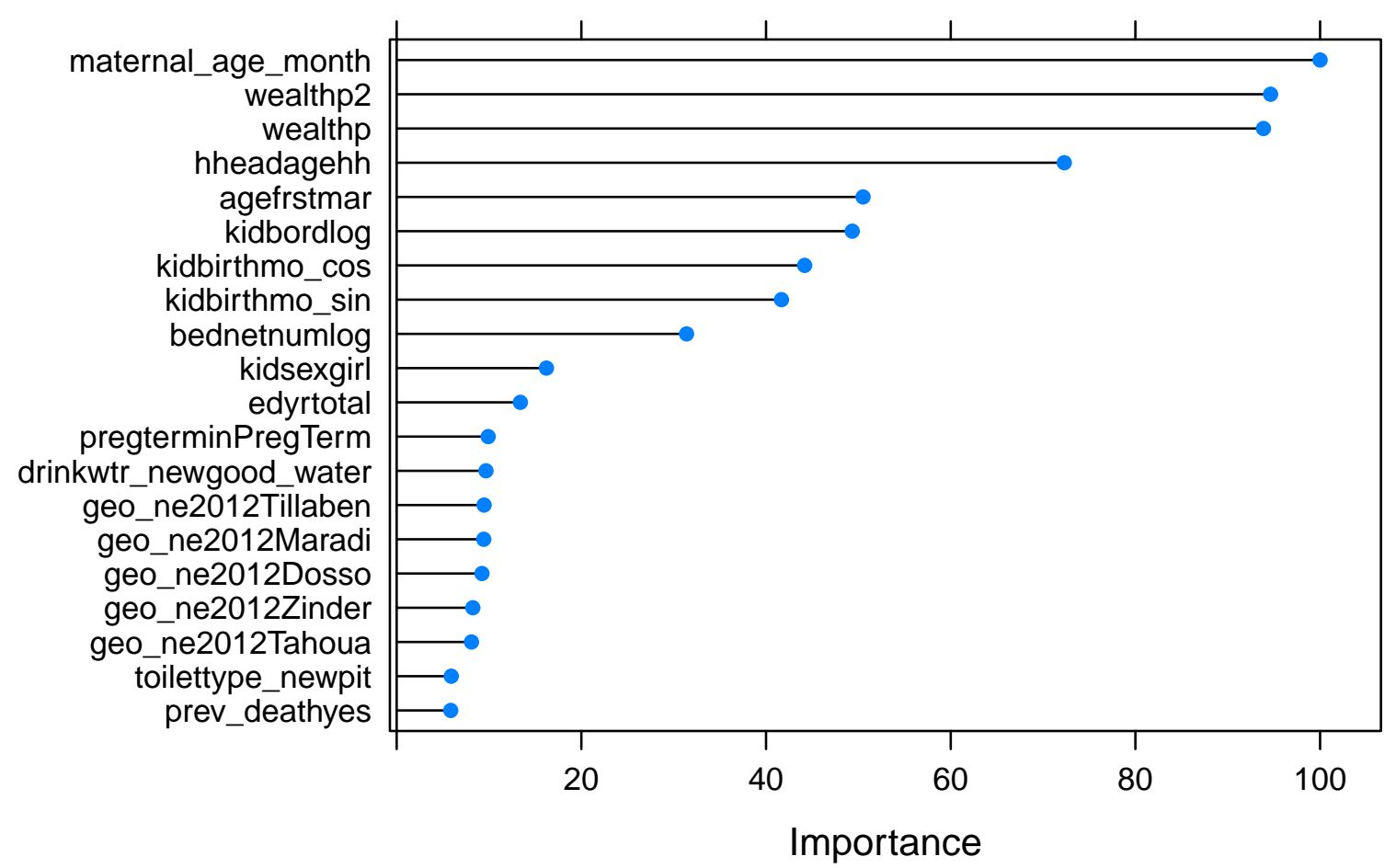

Figure 42: Variable Importance Plot generated by the Random Forest model

\section{B.15.6 Variable Importance Plot}

In this section we will show some measures to give some indication of the important variables necessary for predicting infant mortality. 
medRxiv preprint doi: https://doi.org/10.1101/2021.07.20.21260818; this version posted July 23, 2021. The copyright holder for this preprint (which was not certified by peer review) is the author/funder, who has granted medRxiv a license to display the preprint in perpetuity.

All rights reserved. No reuse allowed without permission.

\section{B.15.7 Elastic Net Coefficients}

Table 120: Standardized coefficients from the optimal elastic net model after 10-fold crossvalidation.

\begin{tabular}{|l|c|c|}
\hline & Coefficients & Standardized Coefficients \\
\hline (Intercept) & -2.654 & -2.654 \\
\hline pregterminPregTerm & 0.1776 & 0.0649 \\
\hline agefrstmar & - & - \\
\hline hheadagehh & - & - \\
\hline urbanRural & 0.2284 & 0.0943 \\
\hline kidsexgirl & - & - \\
\hline hheadsexfemale & -0.0029 & $-9 \mathrm{e}-04$ \\
\hline edyrtotal & -0.0107 & -0.0273 \\
\hline drinkwtr_newgood_water & -0.2188 & -0.1032 \\
\hline floor_newsafe_floor & -0.0835 & -0.0282 \\
\hline maternal_age_month & $-6 \mathrm{e}-04$ & -0.0499 \\
\hline cookfuel_newclean_fuel & - & - \\
\hline toilettype_newpit & - & - \\
\hline toilettype_newunimproved & - & - \\
\hline bednetnumlog & - & - \\
\hline prev_deathyes & 0.3652 & 0.1591 \\
\hline prev_death_fullyes & 0.1226 & 0.0589 \\
\hline kidbordlog & -0.061 & -0.0431 \\
\hline kidbirthmo_sin & -0.0559 & -0.0402 \\
\hline kidbirthmo_cos & - & -0.185 \\
\hline geo_ne2012Diffa & -0.644 & -0.00298 \\
\hline geo_ne2012Dosso & 0.0857 & - \\
\hline geo_ne2012Maradi & 0.0059 & - \\
\hline geo_ne2012Tahoua & - & - \\
\hline geo_ne2012Tillaben & 0.1678 & - \\
\hline geo_ne2012Zinder & - & - \\
\hline geo_ne2012Niamey & 0.0037 & - \\
\hline wealthp & - & - \\
\hline wealthp2 & - & - \\
\hline
\end{tabular}


medRxiv preprint doi: https://doi.org/10.1101/2021.07.20.21260818; this version posted July 23, 2021. The copyright holder for this preprint (which was not certified by peer review) is the author/funder, who has granted medRxiv a license to display the preprint in perpetuity.

All rights reserved. No reuse allowed without permission.

\section{B.16 Nigeria}

This sample was taken in the year 2013. There are 23559 observations in the dataset, and 18848 observations were used for the training set. Also, 663 observations were removed due to missing values. Finally, there were 1686 deaths for the full dataset, and 1349 deaths in the training set. 
medRxiv preprint doi: https://doi.org/10.1101/2021.07.20.21260818; this version posted July 23, 2021. The copyright holder for this preprint (which was not certified by peer review) is the author/funder, who has granted medRxiv a license to display the preprint in perpetuity.

All rights reserved. No reuse allowed without permission. 


\section{B.16.1 Variables Used}

Table 121: Variables included in the model for Nigeria

\begin{tabular}{|c|c|}
\hline Variable & Type \\
\hline mortality.under $12 \mathrm{~m}$ & categorical \\
\hline pregtermin & categorical \\
\hline agefrstmar & numeric \\
\hline hheadagehh & numeric \\
\hline urban & categorical \\
\hline kidsex & categorical \\
\hline hheadsex & categorical \\
\hline edyrtotal & numeric \\
\hline drinkwtr_new & categorical \\
\hline floor_new & categorical \\
\hline maternal_age_month & numeric \\
\hline cookfuel_new & categorical \\
\hline toilettype_new & categorical \\
\hline religion_new & categorical \\
\hline bednetnumlog & numeric \\
\hline prev_death & categorical \\
\hline prev_death_full & categorical \\
\hline malaria_new & numeric \\
\hline kidbordlog & numeric \\
\hline kidbirthmo_sin & numeric \\
\hline kidbirthmo_cos & numeric \\
\hline geo_ng2013 & categorical \\
\hline wealthp & numeric \\
\hline wealthp2 & numeric \\
\hline
\end{tabular}




\section{B.16.2 Mortality Breakdown by Wealth Quintile}

\section{B.16.3 Optimal Parameters}

These are the algorithm parameters selected after cross-validation:

Table 122: Optimal Parameters for the Elastic Net algorithm

\begin{tabular}{|c|c|}
\hline alpha & lambda \\
\hline 0.1642857 & 0.0044743 \\
\hline
\end{tabular}

Table 123: Optimal Parameters for the Random Forest algorithm

\begin{tabular}{|c|}
\hline mtry \\
\hline 9 \\
\hline
\end{tabular}

Table 124: Optimal Parameters for the XGB algorithm

\begin{tabular}{|c|c|c|c|c|c|c|}
\hline nrounds & max_depth & eta & gamma & colsample_bytree & min_child_weight & subsample \\
\hline 50 & 1 & 0.4 & 0 & 0.8 & 1 & 1 \\
\hline
\end{tabular}

Table 125: Optimal Parameters for the KRLS algorithm

\begin{tabular}{|c|c|c|c|}
\hline loss & epsilon & $\mathbf{b}$ & lambda \\
\hline logistic & 0.005 & 60 & 0.0001595 \\
\hline
\end{tabular}


medRxiv preprint doi: https://doi.org/10.1101/2021.07.20.21260818; this version posted July 23, 2021. The copyright holder for this preprint (which was not certified by peer review) is the author/funder, who has granted medRxiv a license to display the preprint in perpetuity.

\section{B.16.4 Table of Results}

Table 126: Manual Cross-Validation Results

\begin{tabular}{|c|c|c|c|c|c|c|c|c|c|}
\hline Algorithm & Recall 10 & Recall 20 & ROC & AUC & MRD & MRR & MSE & F1 & Efficiency Gain \\
\hline Ensemble & 0.199 & 0.339 & 0.634 & 0.127 & 0.017 & 1.227 & 0.065 & 0.922 & 1.604 \\
\hline KRLS & 0.186 & 0.332 & 0.623 & 0.108 & 0.012 & 1.164 & 0.066 & 0.921 & 1.502 \\
\hline Elastic Net & 0.173 & 0.338 & 0.621 & 0.108 & 0.012 & 1.171 & 0.066 & 0.920 & 1.401 \\
\hline Random Forest & 0.175 & 0.294 & 0.603 & 0.117 & 0.031 & 1.375 & 0.067 & 0.920 & 1.413 \\
\hline Wealth & 0.124 & 0.251 & 0.568 & 0.087 & 0.223 & 1.934 & 0.993 & 0.916 & 1.000 \\
\hline XGB & 0.189 & 0.342 & 0.622 & 0.113 & 0.012 & 1.175 & 0.066 & 0.921 & 1.526 \\
\hline
\end{tabular}

Table 127: Distribution of individuals in the top risk decile of each algorithm among each wealth decile

\begin{tabular}{l|r|r|r|r|c}
\hline Wealth Decile & Elastic Net & Ensemble & KRLS & Random Forest & XGB \\
\hline 1 & 0.211 & 0.218 & 0.231 & 0.190 & 0.215 \\
\hline 2 & 0.221 & 0.193 & 0.240 & 0.137 & 0.222 \\
\hline 3 & 0.180 & 0.171 & 0.181 & 0.140 & 0.200 \\
\hline 4 & 0.155 & 0.170 & 0.163 & 0.153 & 0.168 \\
\hline 5 & 0.097 & 0.079 & 0.087 & 0.086 & 0.081 \\
\hline 6 & 0.049 & 0.050 & 0.046 & 0.071 & 0.038 \\
\hline 7 & 0.039 & 0.049 & 0.030 & 0.070 & 0.030 \\
\hline 8 & 0.029 & 0.036 & 0.015 & 0.059 & 0.031 \\
\hline 9 & 0.011 & 0.016 & 0.003 & 0.041 & 0.012 \\
\hline 10 & 0.006 & 0.019 & 0.003 & 0.052 & 0.003 \\
\hline
\end{tabular}

\section{B.16.5 Performance Plots}

Below are various plots showing the performance for each model considered in Nigeria. 
medRxiv preprint doi: https://doi.org/10.1101/2021.07.20.21260818; this version posted July 23, 2021. The copyright holder for this preprint (which was not certified by peer review) is the author/funder, who has granted medRxiv a license to display the preprint in perpetuity. All rights reserved. No reuse allowed without permission.

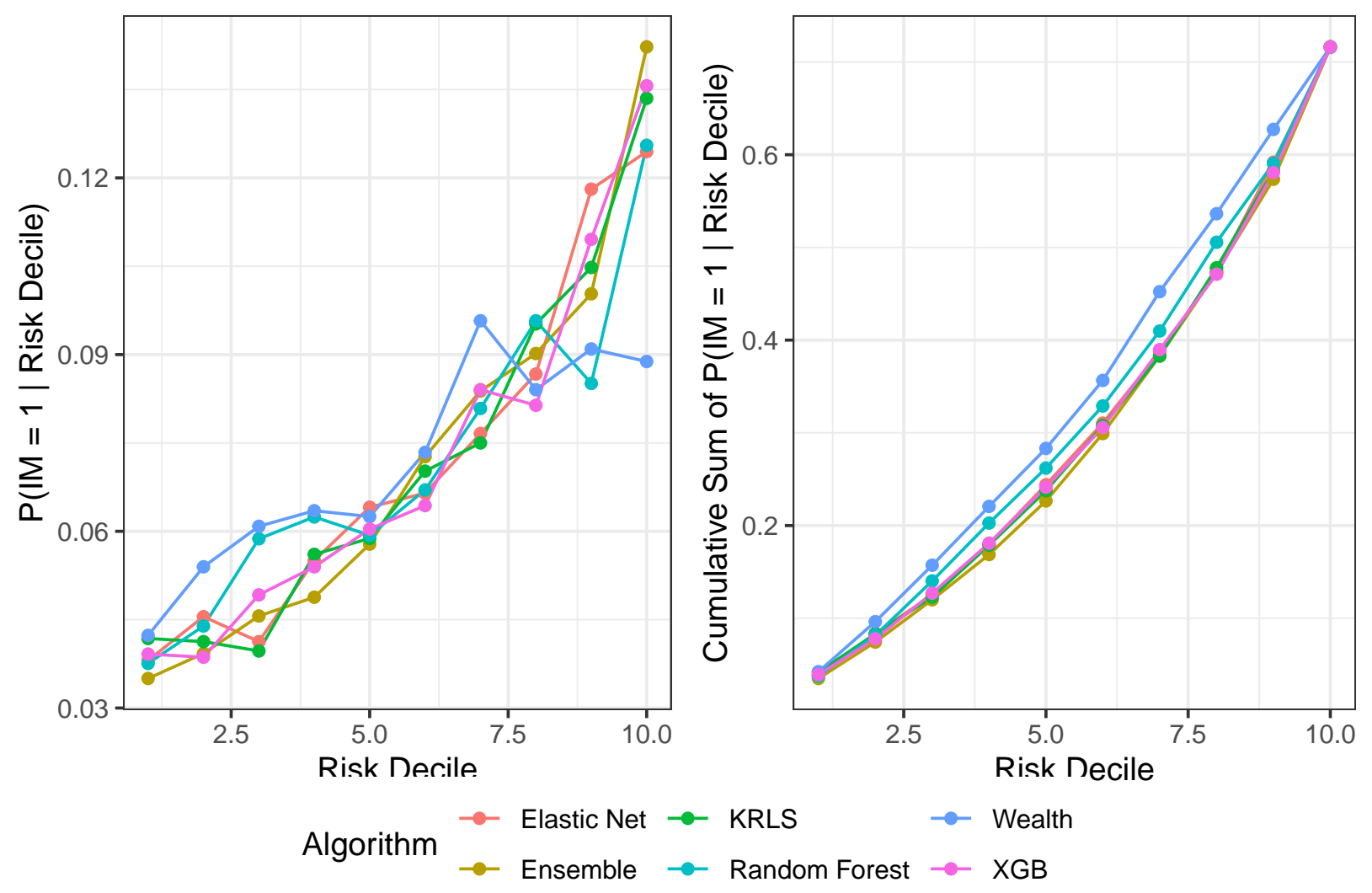

Figure 43: Probability of a mortality given that the observation is in a particular risk decile 
medRxiv preprint doi: https://doi.org/10.1101/2021.07.20.21260818; this version posted July 23, 2021. The copyright holder for this preprint (which was not certified by peer review) is the author/funder, who has granted medRxiv a license to display the preprint in perpetuity. All rights reserved. No reuse allowed without permission.

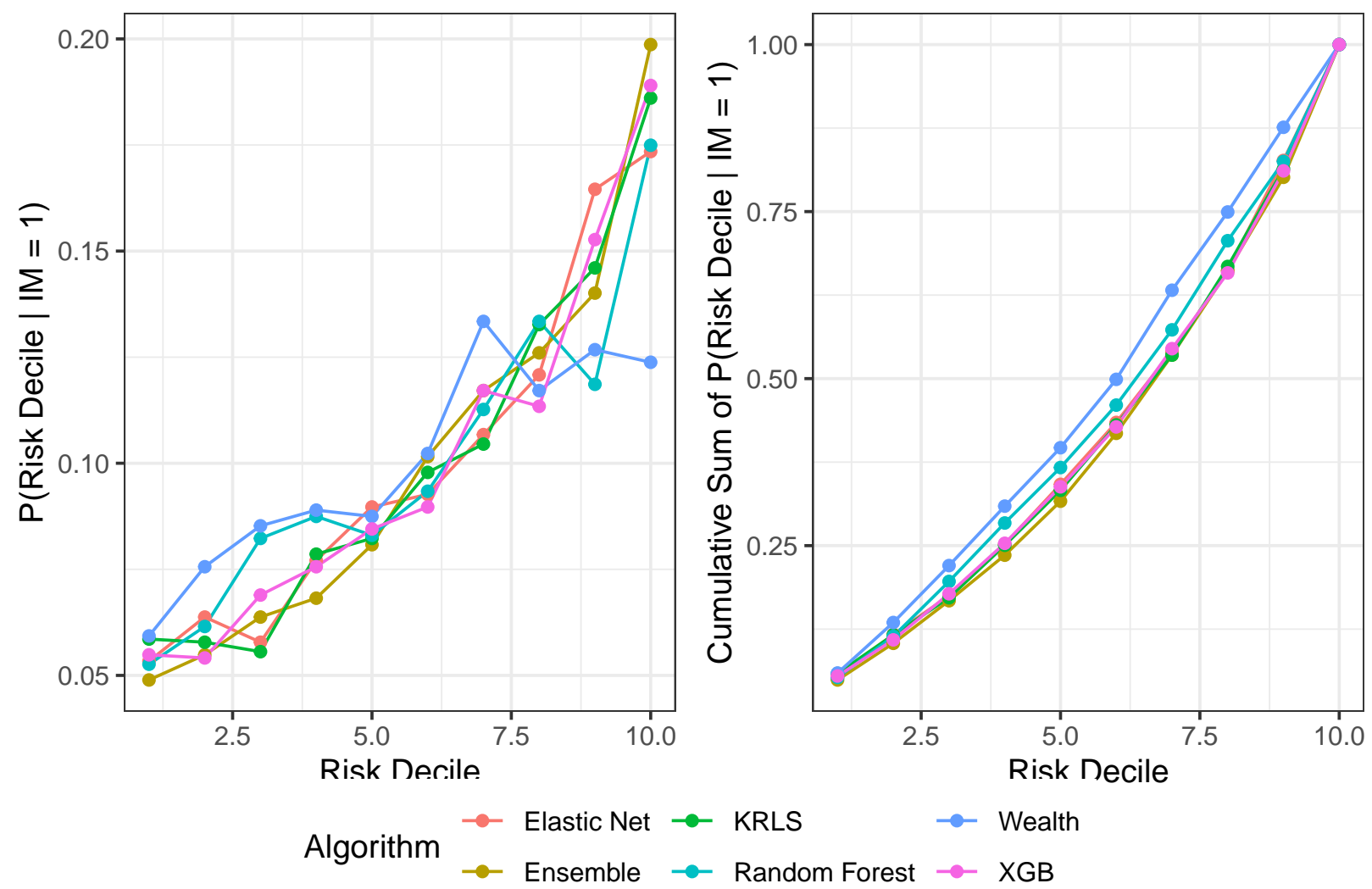

Figure 44: Probability of membership in a particular risk decile given a mortality 


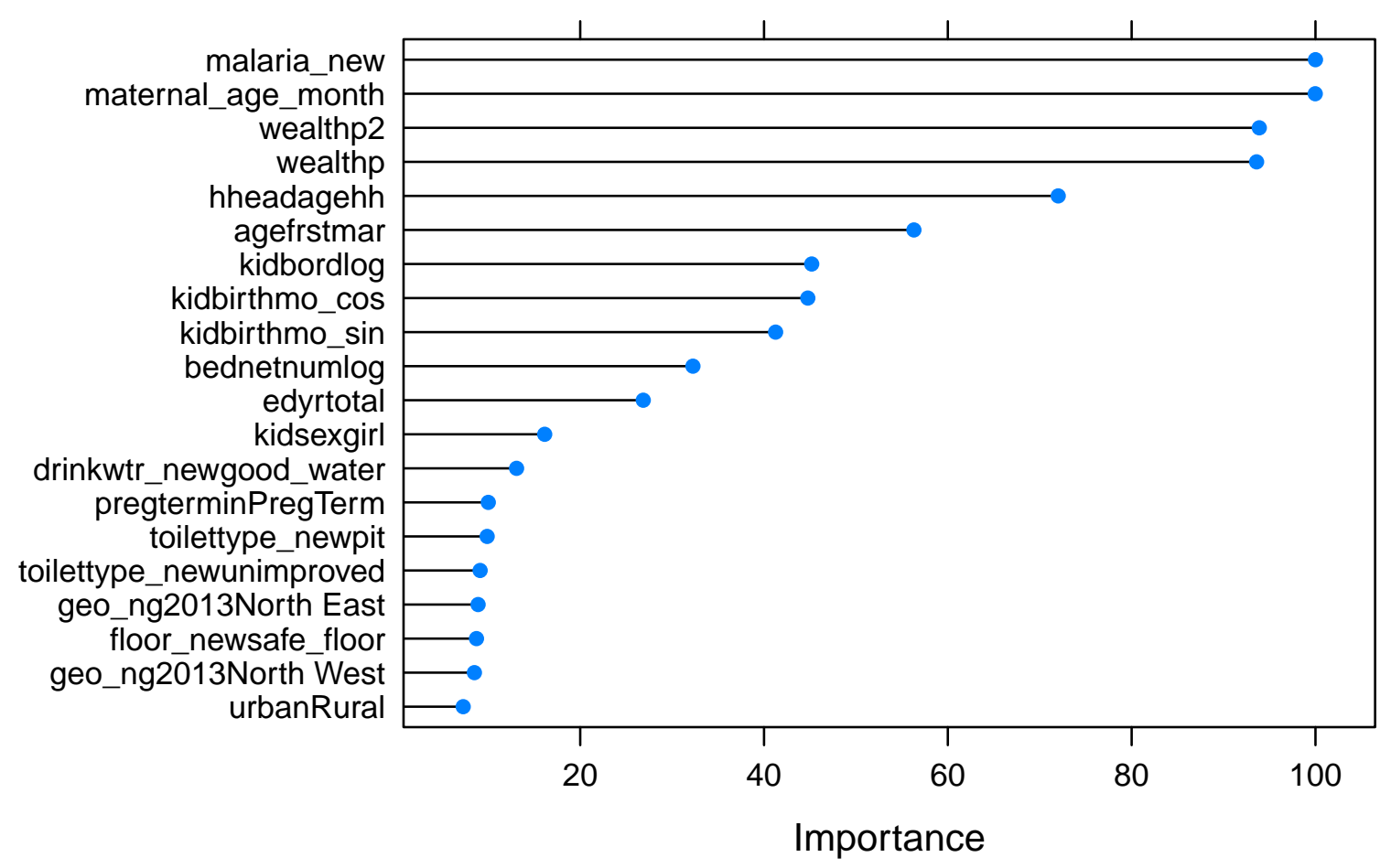

Figure 45: Variable Importance Plot generated by the Random Forest model

\section{B.16.6 Variable Importance Plot}

In this section we will show some measures to give some indication of the important variables necessary for predicting infant mortality. 
medRxiv preprint doi: https://doi.org/10.1101/2021.07.20.21260818; this version posted July 23, 2021. The copyright holder for this preprint (which was not certified by peer review) is the author/funder, who has granted medRxiv a license to display the preprint in perpetuity.

All rights reserved. No reuse allowed without permission.

\section{B.16.7 Elastic Net Coefficients}

Table 128: Standardized coefficients from the optimal elastic net model after 10-fold crossvalidation.

\begin{tabular}{|c|c|c|}
\hline & Coefficients & Standardized Coefficients \\
\hline (Intercept) & -2.6029 & -2.6029 \\
\hline pregterminPregTerm & 0.2537 & 0.0809 \\
\hline agefrstmar & 0.0095 & 0.042 \\
\hline hheadagehh & - & - \\
\hline urbanRural & 0.1704 & 0.0799 \\
\hline kidsexgirl & -0.1129 & -0.0564 \\
\hline hheadsexfemale & -0.0068 & -0.002 \\
\hline edyrtotal & -0.02 & -0.1048 \\
\hline drinkwtr_newgood_water & -0.0115 & -0.0057 \\
\hline floor_newsafe_floor & -0.129 & -0.0642 \\
\hline maternal_age_month & $-6 e-04$ & -0.0518 \\
\hline cookfuel_newclean_fuel & - & - \\
\hline toilettype_newpit & - & - \\
\hline toilettype_newunimproved & - & - \\
\hline religion_newChristian & - & - \\
\hline religion_newHindu & - & - \\
\hline religion_newMuslim & - & - \\
\hline religion_newOther & -0.0323 & -0.004 \\
\hline bednetnumlog & - & - \\
\hline prev_deathyes & 0.4483 & 0.1862 \\
\hline prev_death_fullyes & 0.282 & 0.1308 \\
\hline malaria_new & 0.1074 & 0.0116 \\
\hline kidbordlog & -0.1325 & -0.0943 \\
\hline kidbirthmo_sin & -0.0957 & -0.069 \\
\hline kidbirthmo_cos & -0.0238 & -0.0164 \\
\hline geo_ng2013North East & - & - \\
\hline geo_ng2013North West & 0.1319 & 0.0613 \\
\hline geo_ng2013South East & 0.3659 & 0.1024 \\
\hline geo_ng2013South South & -0.0312 & -0.0099 \\
\hline geo_ng2013South West & - & - \\
\hline wealthp & - & - \\
\hline wealthp2 & -0.1199 & -0.0357 \\
\hline
\end{tabular}


medRxiv preprint doi: https://doi.org/10.1101/2021.07.20.21260818; this version posted July 23, 2021. The copyright holder for this preprint (which was not certified by peer review) is the author/funder, who has granted medRxiv a license to display the preprint in perpetuity.

All rights reserved. No reuse allowed without permission.

\section{B.17 Rwanda}

This sample was taken in the year 2014. There are 5350 observations in the dataset, and 4281 observations were used for the training set. Also, 705 observations were removed due to missing values. Finally, there were 168 deaths for the full dataset, and 135 deaths in the training set. 
medRxiv preprint doi: https://doi.org/10.1101/2021.07.20.21260818; this version posted July 23, 2021. The copyright holder for this preprint (which was not certified by peer review) is the author/funder, who has granted medRxiv a license to display the preprint in perpetuity.

All rights reserved. No reuse allowed without permission. 


\section{B.17.1 Variables Used}

Table 129: Variables included in the model for Rwanda

\begin{tabular}{|c|c|}
\hline Variable & Type \\
\hline mortality.under $12 \mathrm{~m}$ & categorical \\
\hline pregtermin & categorical \\
\hline agefrstmar & numeric \\
\hline hheadagehh & numeric \\
\hline urban & categorical \\
\hline kidsex & categorical \\
\hline hheadsex & categorical \\
\hline edyrtotal & numeric \\
\hline drinkwtr_new & categorical \\
\hline floor_new & categorical \\
\hline maternal_age_month & numeric \\
\hline toilettype_new & categorical \\
\hline religion_new & categorical \\
\hline bednetnumlog & numeric \\
\hline prev_death & categorical \\
\hline prev_death_full & categorical \\
\hline malaria_new & numeric \\
\hline kidbordlog & numeric \\
\hline kidbirthmo_sin & numeric \\
\hline kidbirthmo_cos & numeric \\
\hline geo_rw2014 & categorical \\
\hline wealthp & numeric \\
\hline wealthp2 & numeric \\
\hline
\end{tabular}




\section{B.17.2 Mortality Breakdown by Wealth Quintile}

\section{B.17.3 Optimal Parameters}

These are the algorithm parameters selected after cross-validation:

Table 130: Optimal Parameters for the Elastic Net algorithm

\begin{tabular}{|c|c|}
\hline alpha & lambda \\
\hline 1 & 0.0048172 \\
\hline
\end{tabular}

Table 131: Optimal Parameters for the Random Forest algorithm

\begin{tabular}{|c|}
\hline mtry \\
\hline 2 \\
\hline
\end{tabular}

Table 132: Optimal Parameters for the XGB algorithm

\begin{tabular}{|c|c|c|c|c|c|c|}
\hline nrounds & max_depth & eta & gamma & colsample_bytree & min_child_weight & subsample \\
\hline 50 & 1 & 0.3 & 0 & 0.8 & 1 & 1 \\
\hline
\end{tabular}

Table 133: Optimal Parameters for the KRLS algorithm

\begin{tabular}{|c|c|c|c|}
\hline loss & epsilon & $\mathbf{b}$ & lambda \\
\hline logistic & 0.005 & 56 & 0.0003284 \\
\hline
\end{tabular}


medRxiv preprint doi: https://doi.org/10.1101/2021.07.20.21260818; this version posted July 23, 2021. The copyright holder for this preprint (which was not certified by peer review) is the author/funder, who has granted medRxiv a license to display the preprint in perpetuity.

\section{B.17.4 Table of Results}

Table 134: Manual Cross-Validation Results

\begin{tabular}{|c|c|c|c|c|c|c|c|c|c|}
\hline Algorithm & Recall 10 & Recall 20 & ROC & AUC & MRD & MRR & MSE & F1 & Efficiency Gain \\
\hline Ensemble & 0.186 & 0.320 & 0.618 & 0.068 & 0.005 & 1.172 & 0.030 & 0.936 & 1.251 \\
\hline KRLS & 0.142 & 0.312 & 0.609 & 0.052 & 0.003 & 1.099 & 0.030 & 0.935 & 0.952 \\
\hline Elastic Net & 0.164 & 0.357 & 0.623 & 0.048 & 0.004 & 1.114 & 0.030 & 0.936 & 1.103 \\
\hline Random Forest & 0.207 & 0.296 & 0.578 & 0.068 & 0.010 & 1.517 & 0.031 & 0.938 & 1.387 \\
\hline Wealth & 0.149 & 0.259 & 0.561 & 0.041 & 0.196 & 5.841 & 0.814 & 0.936 & 1.000 \\
\hline XGB & 0.126 & 0.266 & 0.556 & 0.042 & 0.003 & 1.090 & 0.031 & 0.935 & 0.845 \\
\hline
\end{tabular}

Table 135: Distribution of individuals in the top risk decile of each algorithm among each wealth decile

\begin{tabular}{l|r|r|r|r|r}
\hline Wealth Decile & Elastic Net & Ensemble & KRLS & Random Forest & XGB \\
\hline 1 & 0.255 & 0.293 & 0.252 & 0.267 & 0.255 \\
\hline 2 & 0.182 & 0.175 & 0.213 & 0.117 & 0.220 \\
\hline 3 & 0.171 & 0.098 & 0.164 & 0.062 & 0.076 \\
\hline 4 & 0.117 & 0.071 & 0.088 & 0.064 & 0.067 \\
\hline 5 & 0.074 & 0.079 & 0.095 & 0.081 & 0.071 \\
\hline 6 & 0.079 & 0.098 & 0.083 & 0.086 & 0.124 \\
\hline 7 & 0.035 & 0.096 & 0.049 & 0.107 & 0.091 \\
\hline 8 & 0.033 & 0.067 & 0.033 & 0.088 & 0.076 \\
\hline 9 & 0.029 & 0.017 & 0.012 & 0.064 & 0.000 \\
\hline 10 & 0.021 & 0.021 & 0.005 & 0.060 & 0.014 \\
\hline
\end{tabular}

\section{B.17.5 Performance Plots}

Below are various plots showing the performance for each model considered in Rwanda. 
medRxiv preprint doi: https://doi.org/10.1101/2021.07.20.21260818; this version posted July 23, 2021. The copyright holder for this preprint (which was not certified by peer review) is the author/funder, who has granted medRxiv a license to display the preprint in perpetuity. All rights reserved. No reuse allowed without permission.
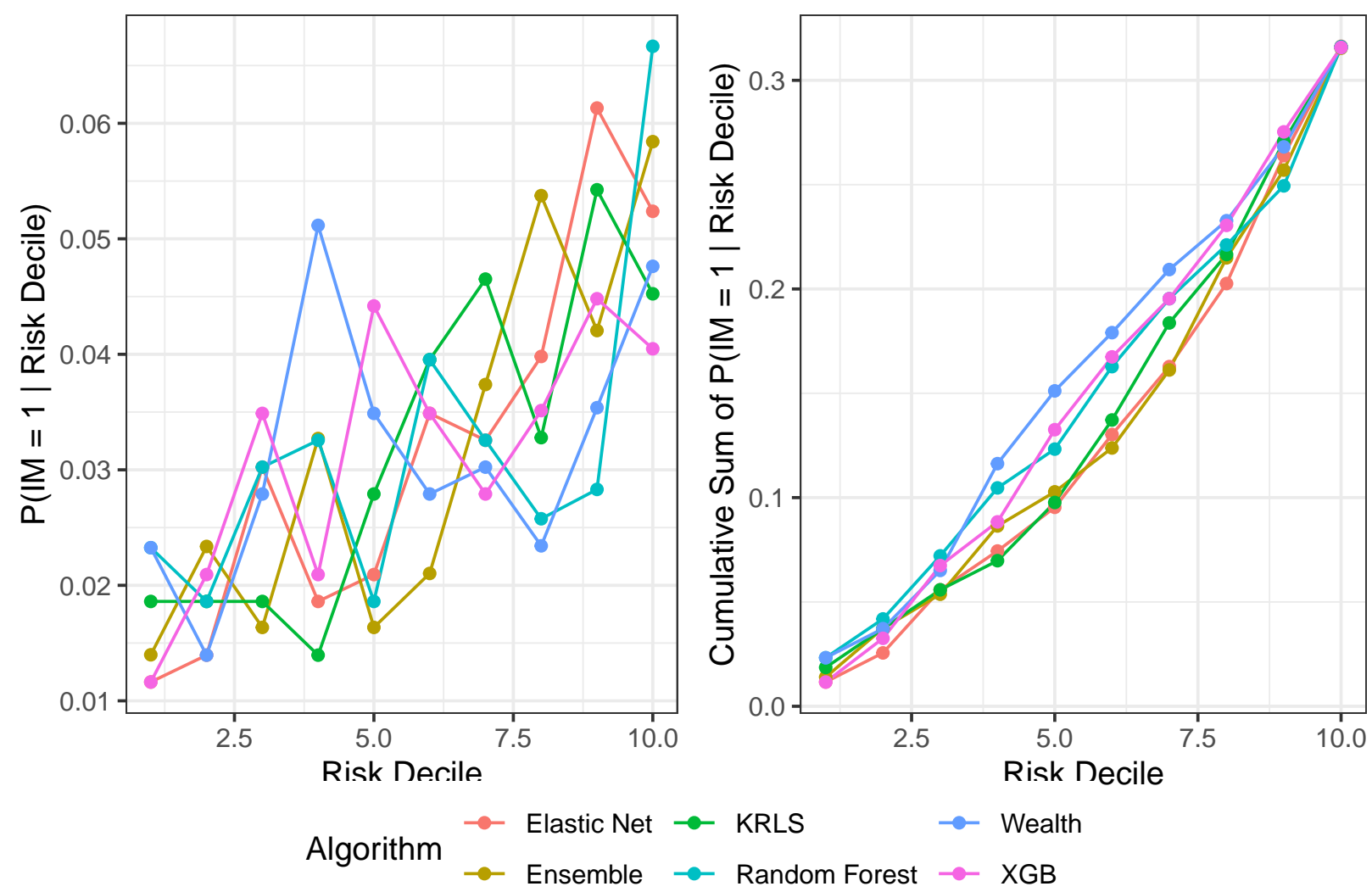

Figure 46: Probability of a mortality given that the observation is in a particular risk decile 
medRxiv preprint doi: https://doi.org/10.1101/2021.07.20.21260818; this version posted July 23, 2021. The copyright holder for this preprint (which was not certified by peer review) is the author/funder, who has granted medRxiv a license to display the preprint in perpetuity. All rights reserved. No reuse allowed without permission.
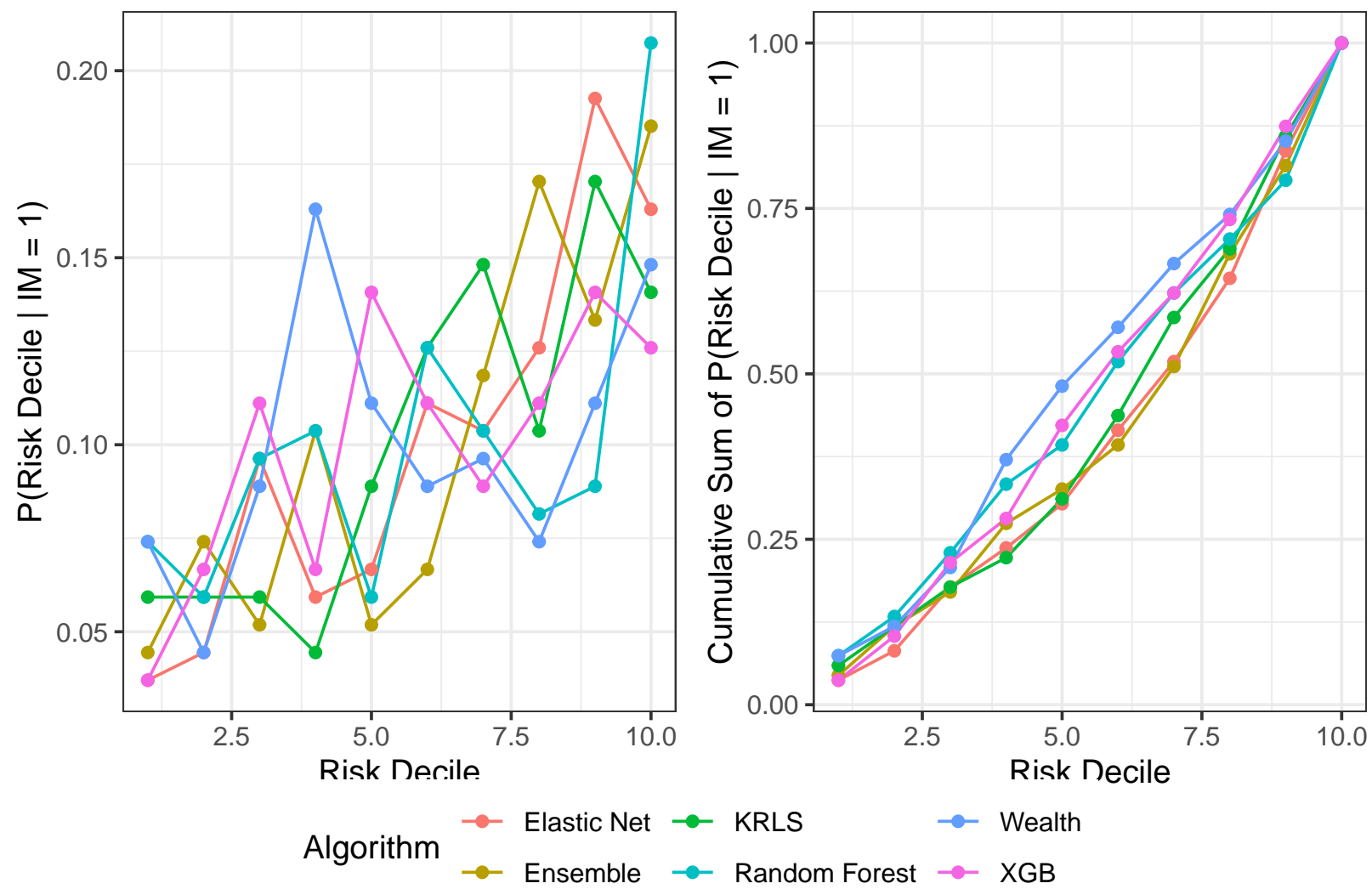

Figure 47: Probability of membership in a particular risk decile given a mortality 


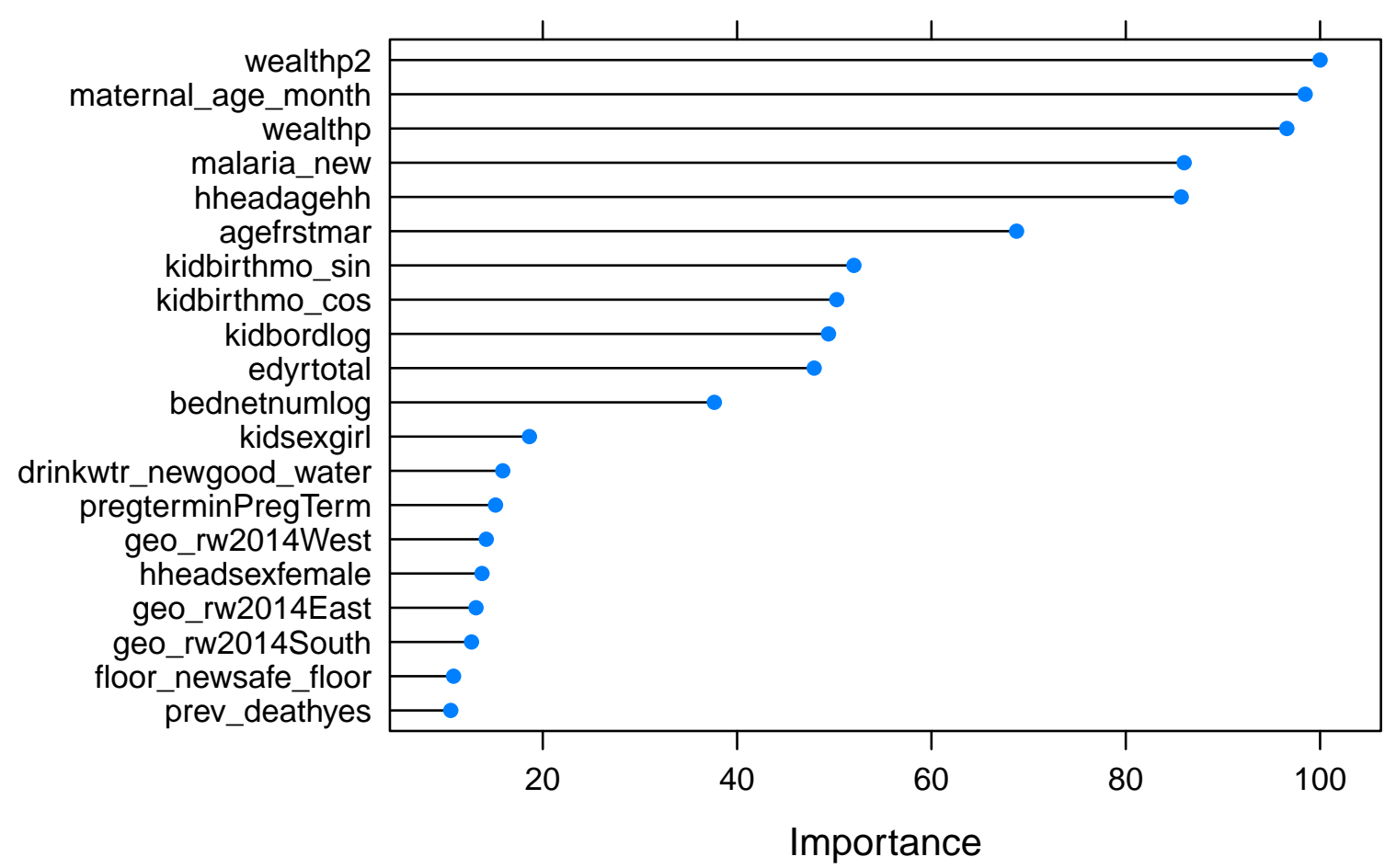

Figure 48: Variable Importance Plot generated by the Random Forest model

\section{B.17.6 Variable Importance Plot}

In this section we will show some measures to give some indication of the important variables necessary for predicting infant mortality. 
medRxiv preprint doi: https://doi.org/10.1101/2021.07.20.21260818; this version posted July 23, 2021. The copyright holder for this preprint (which was not certified by peer review) is the author/funder, who has granted medRxiv a license to display the preprint in perpetuity.

All rights reserved. No reuse allowed without permission.

\section{B.17.7 Elastic Net Coefficients}

Table 136: Standardized coefficients from the optimal elastic net model after 10-fold crossvalidation.

\begin{tabular}{|c|c|c|}
\hline & Coefficients & Standardized Coefficients \\
\hline (Intercept) & -2.9572 & -2.9572 \\
\hline pregterminPregTerm & 0.0025 & $9 \mathrm{e}-04$ \\
\hline agefrstmar & - & - \\
\hline hheadagehh & - & - \\
\hline urbanRural & - & - \\
\hline kidsexgirl & - & - \\
\hline hheadsexfemale & - & - \\
\hline edyrtotal & -0.0044 & -0.0158 \\
\hline drinkwtr_newgood_water & - & - \\
\hline floor_newsafe_floor & - & - \\
\hline maternal_age_month & - & - \\
\hline toilettype_newpit & - & - \\
\hline toilettype_newunimproved & - & - \\
\hline religion_newChristian & - & - \\
\hline religion_newHindu & - & - \\
\hline religion_newMuslim & - & - \\
\hline religion_newOther & - & - \\
\hline bednetnumlog & -0.5674 & -0.2638 \\
\hline prev_deathyes & - & - \\
\hline prev_death_fullyes & 0.148 & 0.0587 \\
\hline malaria_new & - & - \\
\hline kidbordlog & - & - \\
\hline kidbirthmo_sin & - & - \\
\hline kidbirthmo_cos & - & - \\
\hline geo_rw2014South & - & - \\
\hline geo_rw2014West & - & - \\
\hline geo_rw2014North & -0.0035 & -0.0012 \\
\hline geo_rw2014East & - & - \\
\hline wealthp & - & - \\
\hline wealthp2 & - & - \\
\hline
\end{tabular}


medRxiv preprint doi: https://doi.org/10.1101/2021.07.20.21260818; this version posted July 23, 2021. The copyright holder for this preprint (which was not certified by peer review) is the author/funder, who has granted medRxiv a license to display the preprint in perpetuity.

All rights reserved. No reuse allowed without permission.

\section{B.18 Senegal}

This sample was taken in the year 2017. There are 9371 observations in the dataset, and 7497 observations were used for the training set. Also, 214 observations were removed due to missing values. Finally, there were 401 deaths for the full dataset, and 321 deaths in the training set. 
medRxiv preprint doi: https://doi.org/10.1101/2021.07.20.21260818; this version posted July 23, 2021. The copyright holder for this preprint (which was not certified by peer review) is the author/funder, who has granted medRxiv a license to display the preprint in perpetuity.

All rights reserved. No reuse allowed without permission. 


\section{B.18.1 Variables Used}

Table 137: Variables included in the model for Senegal

\begin{tabular}{|l|l|}
\hline Variable & Type \\
\hline mortality.under12m & categorical \\
\hline pregtermin & categorical \\
\hline agefrstmar & numeric \\
\hline hheadagehh & numeric \\
\hline urban & categorical \\
\hline kidsex & categorical \\
\hline hheadsex & categorical \\
\hline edyrtotal & numeric \\
\hline drinkwtr_new & categorical \\
\hline floor_new & categorical \\
\hline maternal_age_month & numeric \\
\hline cookfuel_new & categorical \\
\hline toilettype_new & categorical \\
\hline religion_new & categorical \\
\hline bednetnumlog & numeric \\
\hline prev_death & categorical \\
\hline prev_death_full & categorical \\
\hline kidbordlog & numeric \\
\hline kidbirthmo_sin & numeric \\
\hline kidbirthmo_cos & numeric \\
\hline geo_sn2017 & categorical \\
\hline wealthp & numealthp2 \\
\hline wic
\end{tabular}




\section{B.18.2 Mortality Breakdown by Wealth Quintile}

\section{B.18.3 Optimal Parameters}

These are the algorithm parameters selected after cross-validation:

Table 138: Optimal Parameters for the Elastic Net algorithm

\begin{tabular}{|c|c|}
\hline alpha & lambda \\
\hline 0.1 & 0.0127515 \\
\hline
\end{tabular}

Table 139: Optimal Parameters for the Random Forest algorithm

\begin{tabular}{|c|}
\hline mtry \\
\hline 11 \\
\hline
\end{tabular}

Table 140: Optimal Parameters for the XGB algorithm

\begin{tabular}{|c|c|c|c|c|c|c|}
\hline nrounds & max_depth & eta & gamma & colsample_bytree & min_child_weight & subsample \\
\hline 150 & 5 & 0.3 & 0 & 0.6 & 1 & 1 \\
\hline
\end{tabular}

Table 141: Optimal Parameters for the KRLS algorithm

\begin{tabular}{|c|c|c|c|}
\hline loss & epsilon & $\mathbf{b}$ & lambda \\
\hline logistic & 0.005 & 74 & 0.0002243 \\
\hline
\end{tabular}


medRxiv preprint doi: https://doi.org/10.1101/2021.07.20.21260818; this version posted July 23, 2021. The copyright holder for this preprint (which was not certified by peer review) is the author/funder, who has granted medRxiv a license to display the preprint in perpetuity.

\section{B.18.4 Table of Results}

Table 142: Manual Cross-Validation Results

\begin{tabular}{|c|c|c|c|c|c|c|c|c|c|}
\hline Algorithm & Recall 10 & Recall 20 & ROC & AUC & MRD & MRR & MSE & F1 & Efficiency Gain \\
\hline Ensemble & 0.224 & 0.339 & 0.609 & 0.148 & 0.033 & 1.810 & 0.039 & 0.933 & 2.660 \\
\hline KRLS & 0.165 & 0.315 & 0.581 & 0.075 & 0.004 & 1.100 & 0.041 & 0.931 & 1.957 \\
\hline Elastic Net & 0.149 & 0.299 & 0.580 & 0.071 & 0.004 & 1.101 & 0.041 & 0.930 & 1.773 \\
\hline Random Forest & 0.209 & 0.311 & 0.587 & 0.144 & 0.059 & 2.194 & 0.040 & 0.933 & 2.475 \\
\hline Wealth & 0.084 & 0.203 & 0.535 & 0.045 & 0.106 & 1.687 & 0.956 & 0.927 & 1.000 \\
\hline XGB & 0.209 & 0.333 & 0.595 & 0.121 & 0.064 & 3.297 & 0.041 & 0.933 & 2.476 \\
\hline
\end{tabular}

Table 143: Distribution of individuals in the top risk decile of each algorithm among each wealth decile

\begin{tabular}{l|r|r|r|r|r}
\hline Wealth Decile & Elastic Net & Ensemble & KRLS & Random Forest & XGB \\
\hline 1 & 0.181 & 0.100 & 0.146 & 0.106 & 0.067 \\
\hline 2 & 0.147 & 0.116 & 0.159 & 0.116 & 0.105 \\
\hline 3 & 0.126 & 0.146 & 0.135 & 0.130 & 0.134 \\
\hline 4 & 0.122 & 0.114 & 0.127 & 0.096 & 0.115 \\
\hline 5 & 0.103 & 0.103 & 0.115 & 0.090 & 0.111 \\
\hline 6 & 0.110 & 0.088 & 0.120 & 0.091 & 0.076 \\
\hline 7 & 0.085 & 0.112 & 0.096 & 0.108 & 0.135 \\
\hline 8 & 0.050 & 0.100 & 0.052 & 0.088 & 0.127 \\
\hline 9 & 0.052 & 0.067 & 0.043 & 0.075 & 0.075 \\
\hline 10 & 0.024 & 0.055 & 0.005 & 0.099 & 0.054 \\
\hline
\end{tabular}

\section{B.18.5 Performance Plots}

Below are various plots showing the performance for each model considered in Senegal. 
medRxiv preprint doi: https://doi.org/10.1101/2021.07.20.21260818; this version posted July 23, 2021. The copyright holder for this preprint (which was not certified by peer review) is the author/funder, who has granted medRxiv a license to display the preprint in perpetuity. All rights reserved. No reuse allowed without permission.
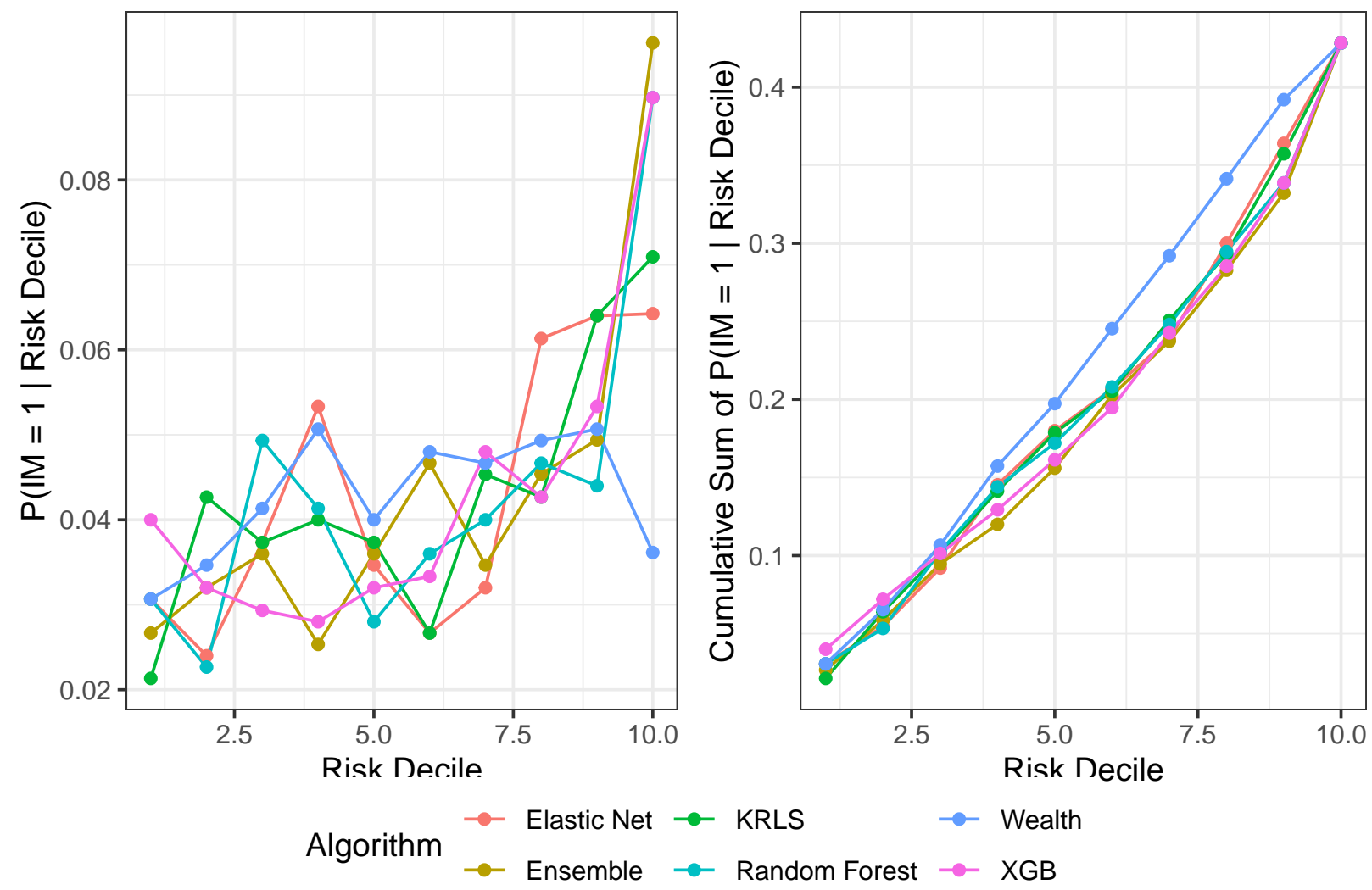

Figure 49: Probability of a mortality given that the observation is in a particular risk decile 
medRxiv preprint doi: https://doi.org/10.1101/2021.07.20.21260818; this version posted July 23, 2021. The copyright holder for this preprint (which was not certified by peer review) is the author/funder, who has granted medRxiv a license to display the preprint in perpetuity. All rights reserved. No reuse allowed without permission.

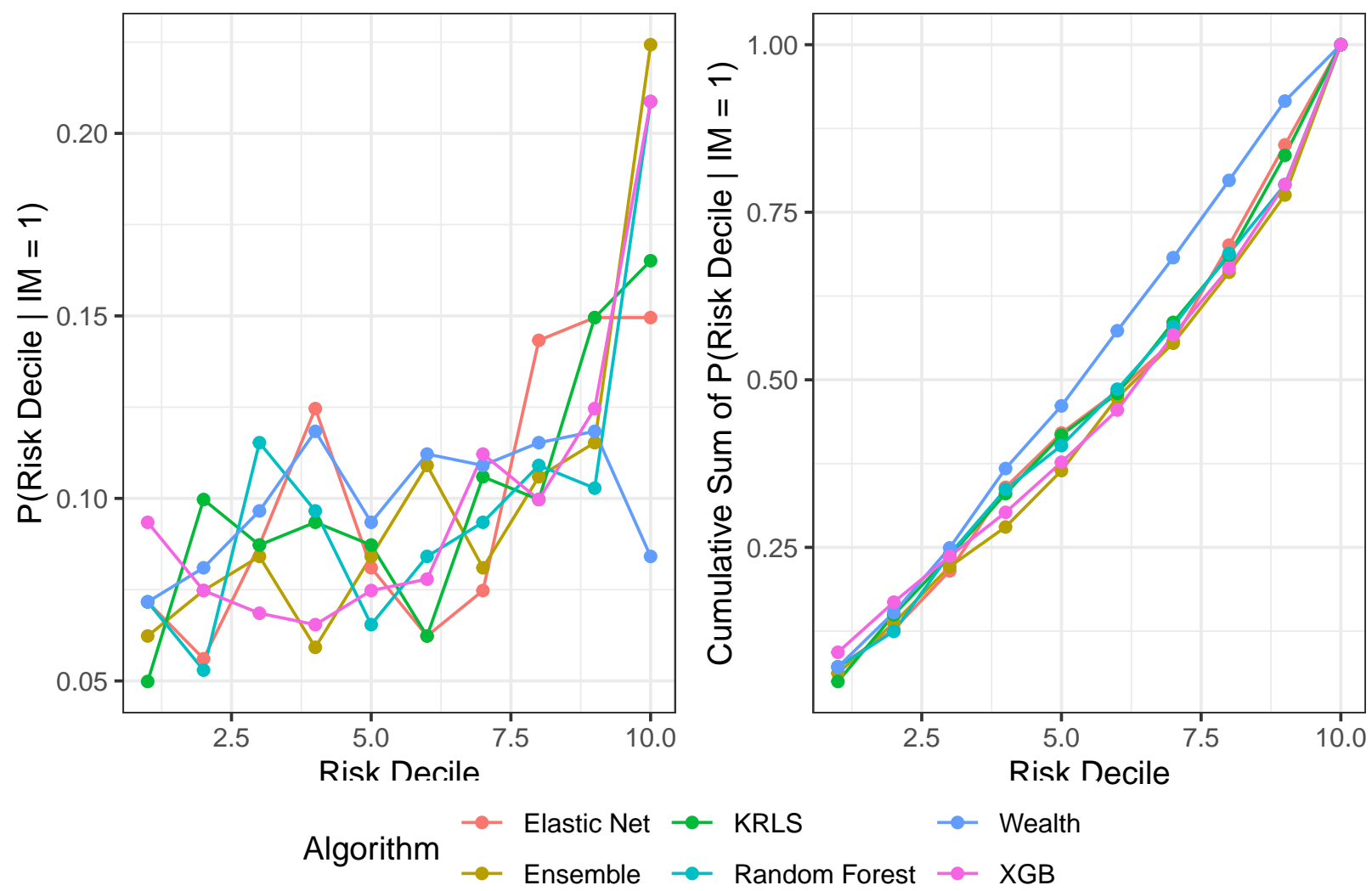

Figure 50: Probability of membership in a particular risk decile given a mortality 


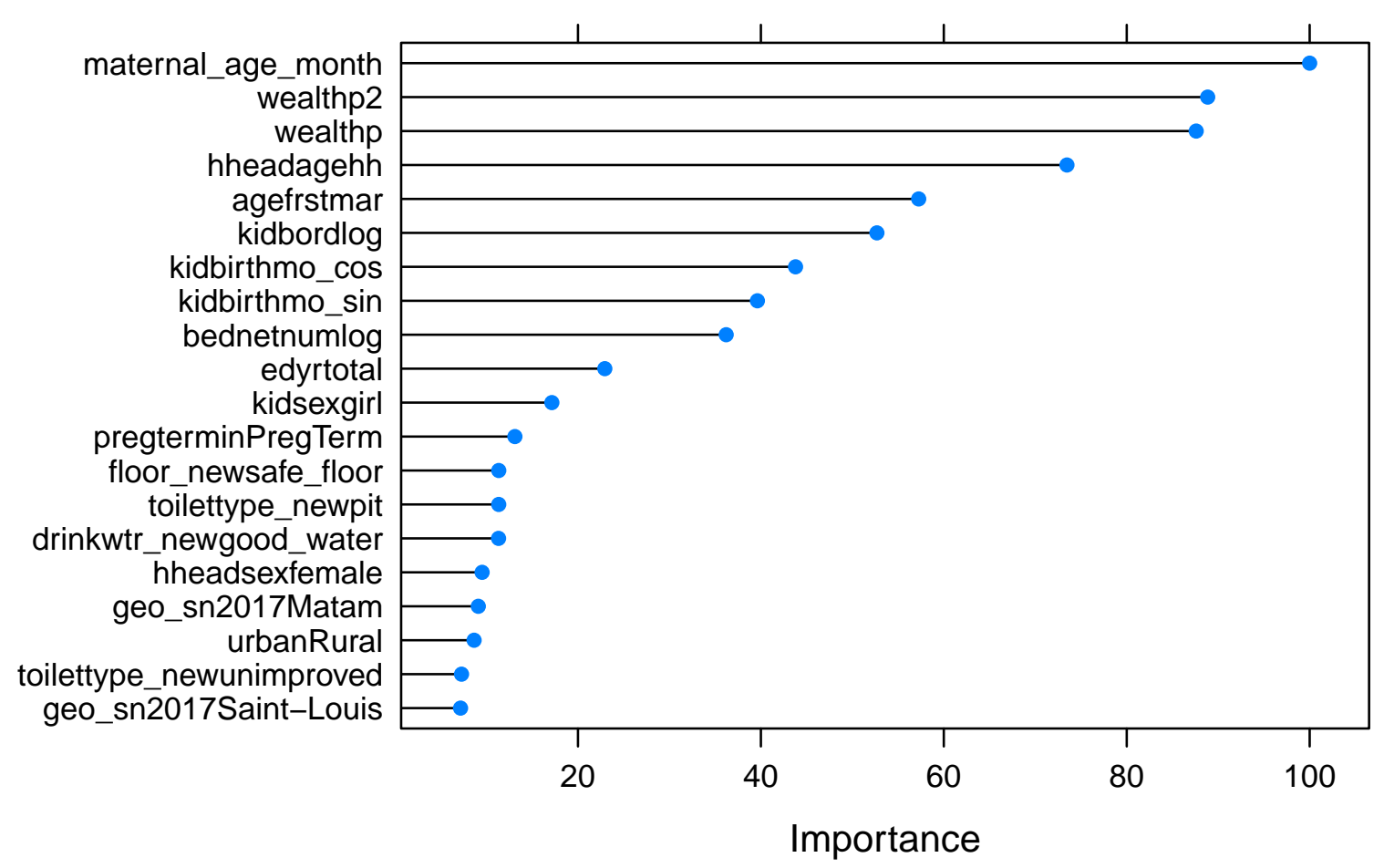

Figure 51: Variable Importance Plot generated by the Random Forest model

\section{B.18.6 Variable Importance Plot}

In this section we will show some measures to give some indication of the important variables necessary for predicting infant mortality. 
medRxiv preprint doi: https://doi.org/10.1101/2021.07.20.21260818; this version posted July 23, 2021. The copyright holder for this preprint (which was not certified by peer review) is the author/funder, who has granted medRxiv a license to display the preprint in perpetuity.

All rights reserved. No reuse allowed without permission.

\section{B.18.7 Elastic Net Coefficients}

Table 144: Standardized coefficients from the optimal elastic net model after 10-fold crossvalidation.

\begin{tabular}{|c|c|c|}
\hline & Coefficients & Standardized Coefficients \\
\hline (Intercept) & -2.8655 & -2.8655 \\
\hline pregterminPregTerm & 0.1831 & 0.0729 \\
\hline agefrstmar & - & - \\
\hline hheadagehh & $-2 \mathrm{e}-04$ & -0.0031 \\
\hline urbanRural & 0.0245 & 0.0116 \\
\hline kidsexgirl & -0.0474 & -0.0237 \\
\hline hheadsexfemale & -0.0105 & -0.0044 \\
\hline edyrtotal & -0.0123 & -0.0432 \\
\hline drinkwtr_newgood_water & -0.0767 & -0.0361 \\
\hline floor_newsafe_floor & -0.1394 & -0.0695 \\
\hline maternal_age_month & $-5 e-04$ & -0.0419 \\
\hline cookfuel_newclean_fuel & - & - \\
\hline toilettype_newpit & - & - \\
\hline toilettype_newunimproved & - & - \\
\hline religion_newChristian & -0.2178 & -0.0313 \\
\hline religion_newHindu & - & - \\
\hline religion_newMuslim & 0.0229 & 0.0033 \\
\hline religion_newOther & 1.2575 & 0.0325 \\
\hline bednetnumlog & -0.0585 & -0.0358 \\
\hline prev_deathyes & 0.3571 & 0.1207 \\
\hline prev_death_fullyes & 0.1783 & 0.0703 \\
\hline kidbordlog & - & - \\
\hline kidbirthmo_sin & 0.0883 & 0.0623 \\
\hline kidbirthmo_cos & -0.1382 & -0.0974 \\
\hline geo_sn2017Ziguinchor & -0.0471 & -0.0095 \\
\hline geo_sn2017Diourbel & - & - \\
\hline geo_sn2017Saint-Louis & 0.1278 & 0.0311 \\
\hline geo_sn2017Tambacounda & -0.0451 & -0.0124 \\
\hline geo_sn2017Kaolack & -0.1627 & -0.0395 \\
\hline geo_sn2017Thiès & 0.0028 & $7 \mathrm{e}-04$ \\
\hline geo_sn2017Louga & 0.2438 & 0.063 \\
\hline geo_sn2017Fatick & -0.0746 & -0.0196 \\
\hline geo_sn2017Kolda & -0.076 & -0.02 \\
\hline geo_sn2017Matam & 0.4622 & 0.1264 \\
\hline geo_sn2017Kaffrine & - & - \\
\hline geo_sn2017Kedougou & - & - \\
\hline geo_sn2017Sedhiou & - & - \\
\hline wealthp & - & - \\
\hline wealthp2 & - & - \\
\hline
\end{tabular}


medRxiv preprint doi: https://doi.org/10.1101/2021.07.20.21260818; this version posted July 23, 2021. The copyright holder for this preprint (which was not certified by peer review) is the author/funder, who has granted medRxiv a license to display the preprint in perpetuity.

All rights reserved. No reuse allowed without permission.

\section{B.19 Tanzania}

This sample was taken in the year 2015. There are 7688 observations in the dataset, and 6152 observations were used for the training set. Also, 319 observations were removed due to missing values. Finally, there were 319 deaths for the full dataset, and 256 deaths in the training set. 


\section{B.19.1 Variables Used}

Table 145: Variables included in the model for Tanzania

\begin{tabular}{|l|l|}
\hline Variable & Type \\
\hline mortality.under12m & categorical \\
\hline pregtermin & categorical \\
\hline agefrstmar & numeric \\
\hline hheadagehh & numeric \\
\hline urban & categorical \\
\hline kidsex & categorical \\
\hline hheadsex & categorical \\
\hline edyrtotal & numeric \\
\hline drinkwtr_new & categorical \\
\hline floor_new & categorical \\
\hline maternal_age_month & numeric \\
\hline toilettype_new & categorical \\
\hline bednetnumlog & numeric \\
\hline prev_death & categorical \\
\hline prev_death_full & categorical \\
\hline kidbordlog & numeric \\
\hline kidbirthmo_sin & numeric \\
\hline kidbirthmo_cos & numeric \\
\hline district & categorical \\
\hline wealthp & numeric \\
\hline wealthp2 & \\
\hline
\end{tabular}




\section{B.19.2 Mortality Breakdown by Wealth Quintile}

\section{B.19.3 Optimal Parameters}

These are the algorithm parameters selected after cross-validation:

Table 146: Optimal Parameters for the Elastic Net algorithm

\begin{tabular}{|c|c|}
\hline alpha & lambda \\
\hline 0.8071429 & 0.0030448 \\
\hline
\end{tabular}

Table 147: Optimal Parameters for the Random Forest algorithm

\begin{tabular}{|c|}
\hline mtry \\
\hline 2 \\
\hline
\end{tabular}

Table 148: Optimal Parameters for the XGB algorithm

\begin{tabular}{|c|c|c|c|c|c|c|}
\hline nrounds & max_depth & eta & gamma & colsample_bytree & min_child_weight & subsample \\
\hline 50 & 1 & 0.3 & 0 & 0.6 & 1 & 0.75 \\
\hline
\end{tabular}

Table 149: Optimal Parameters for the KRLS algorithm

\begin{tabular}{|c|c|c|c|}
\hline loss & epsilon & $\mathbf{b}$ & lambda \\
\hline logistic & 0.005 & 60 & 0.0001249 \\
\hline
\end{tabular}


medRxiv preprint doi: https://doi.org/10.1101/2021.07.20.21260818; this version posted July 23, 2021. The copyright holder for this preprint (which was not certified by peer review) is the author/funder, who has granted medRxiv a license to display the preprint in perpetuity.

\section{B.19.4 Table of Results}

Table 150: Manual Cross-Validation Results

\begin{tabular}{|c|c|c|c|c|c|c|c|c|c|}
\hline Algorithm & Recall 10 & Recall 20 & ROC & AUC & MRD & MRR & MSE & F1 & Efficiency Gain \\
\hline Ensemble & 0.188 & 0.262 & 0.582 & 0.077 & 0.005 & 1.145 & 0.04 & 0.932 & 2.169 \\
\hline KRLS & 0.144 & 0.254 & 0.549 & 0.076 & 0.004 & 1.098 & 0.04 & 0.931 & 1.667 \\
\hline Elastic Net & 0.121 & 0.226 & 0.561 & 0.066 & 0.002 & 1.054 & 0.04 & 0.930 & 1.397 \\
\hline Random Forest & 0.180 & 0.309 & 0.584 & 0.072 & 0.011 & 1.459 & 0.04 & 0.932 & 2.080 \\
\hline Wealth & 0.086 & 0.161 & 0.545 & 0.039 & -0.128 & 0.251 & 0.95 & 0.928 & 1.000 \\
\hline XGB & 0.164 & 0.285 & 0.560 & 0.065 & 0.005 & 1.110 & 0.04 & 0.932 & 1.895 \\
\hline
\end{tabular}

Table 151: Distribution of individuals in the top risk decile of each algorithm among each wealth decile

\begin{tabular}{l|r|r|r|r|c}
\hline Wealth Decile & Elastic Net & Ensemble & KRLS & Random Forest & XGB \\
\hline 1 & 0.044 & 0.052 & 0.026 & 0.107 & 0.054 \\
\hline 2 & 0.050 & 0.059 & 0.031 & 0.065 & 0.067 \\
\hline 3 & 0.048 & 0.061 & 0.048 & 0.057 & 0.084 \\
\hline 4 & 0.056 & 0.057 & 0.041 & 0.069 & 0.092 \\
\hline 5 & 0.075 & 0.062 & 0.062 & 0.061 & 0.077 \\
\hline 6 & 0.074 & 0.052 & 0.074 & 0.051 & 0.054 \\
\hline 7 & 0.099 & 0.107 & 0.098 & 0.098 & 0.081 \\
\hline 8 & 0.120 & 0.143 & 0.149 & 0.120 & 0.138 \\
\hline 9 & 0.184 & 0.146 & 0.234 & 0.118 & 0.115 \\
\hline 10 & 0.249 & 0.267 & 0.236 & 0.254 & 0.238 \\
\hline
\end{tabular}

\section{B.19.5 Performance Plots}

Below are various plots showing the performance for each model considered in Tanzania. 
medRxiv preprint doi: https://doi.org/10.1101/2021.07.20.21260818; this version posted July 23, 2021. The copyright holder for this preprint (which was not certified by peer review) is the author/funder, who has granted medRxiv a license to display the preprint in perpetuity. All rights reserved. No reuse allowed without permission.

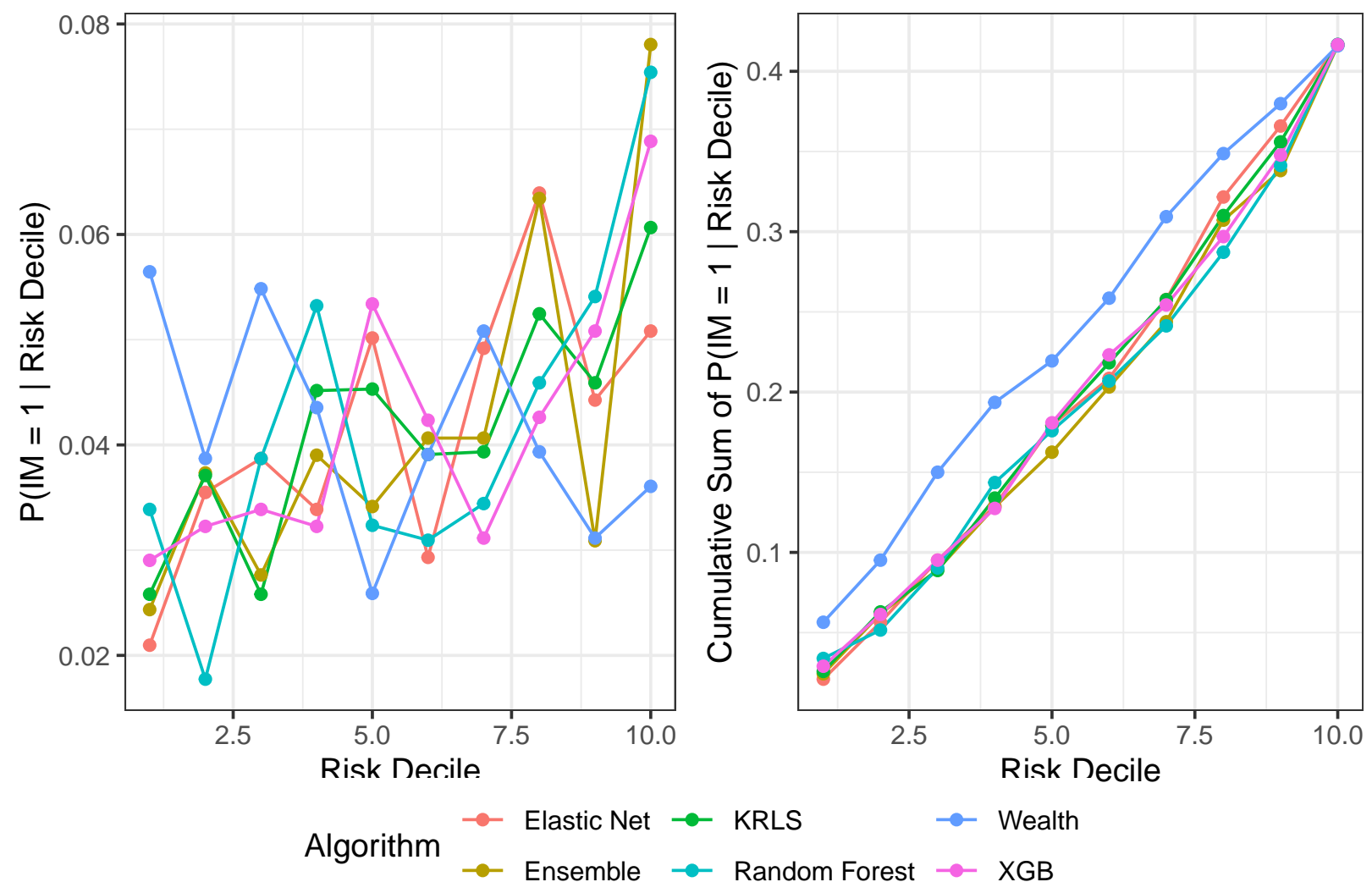

Figure 52: Probability of a mortality given that the observation is in a particular risk decile 
medRxiv preprint doi: https://doi.org/10.1101/2021.07.20.21260818; this version posted July 23, 2021. The copyright holder for this preprint (which was not certified by peer review) is the author/funder, who has granted medRxiv a license to display the preprint in perpetuity. All rights reserved. No reuse allowed without permission.
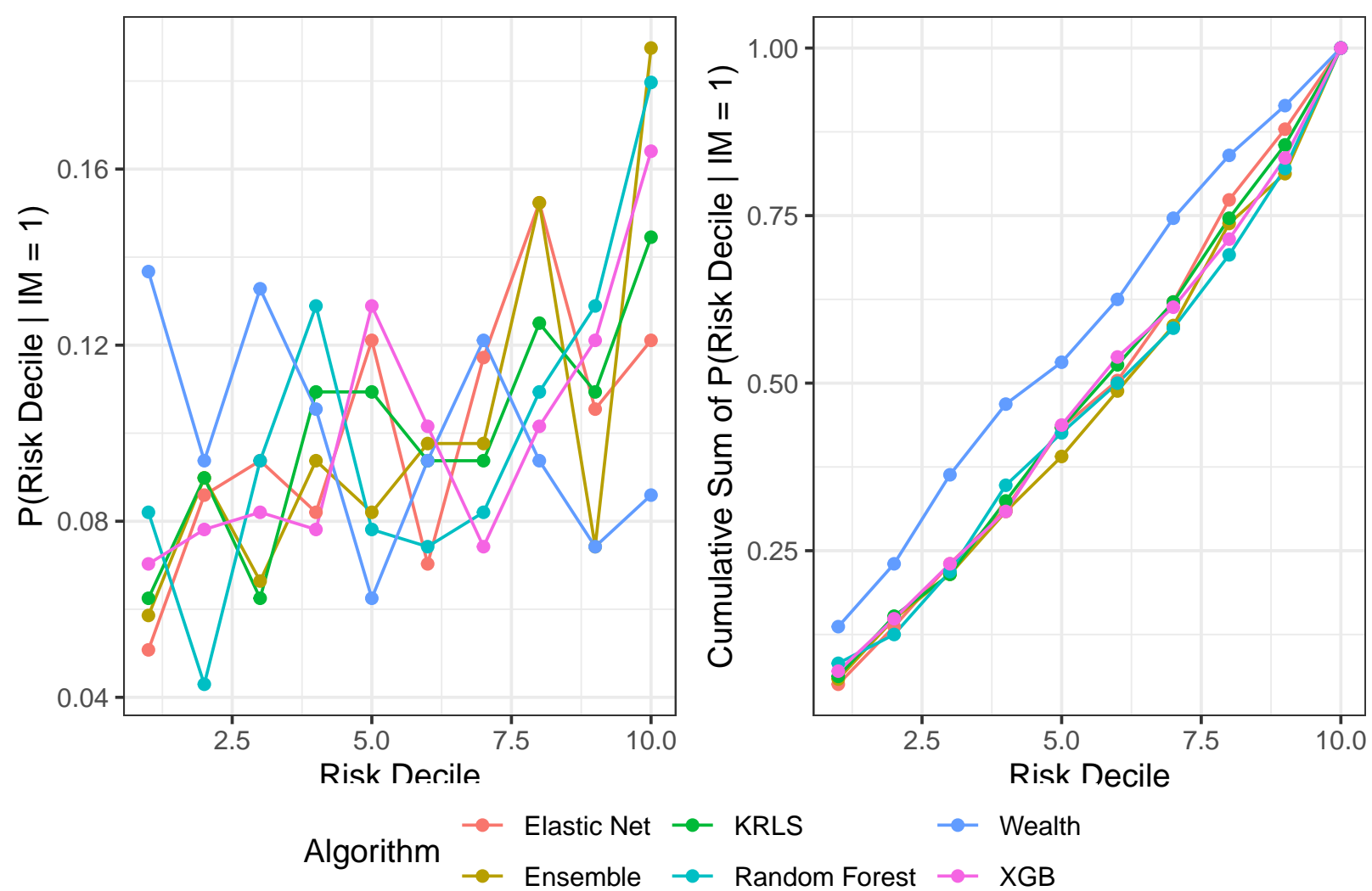

Figure 53: Probability of membership in a particular risk decile given a mortality 


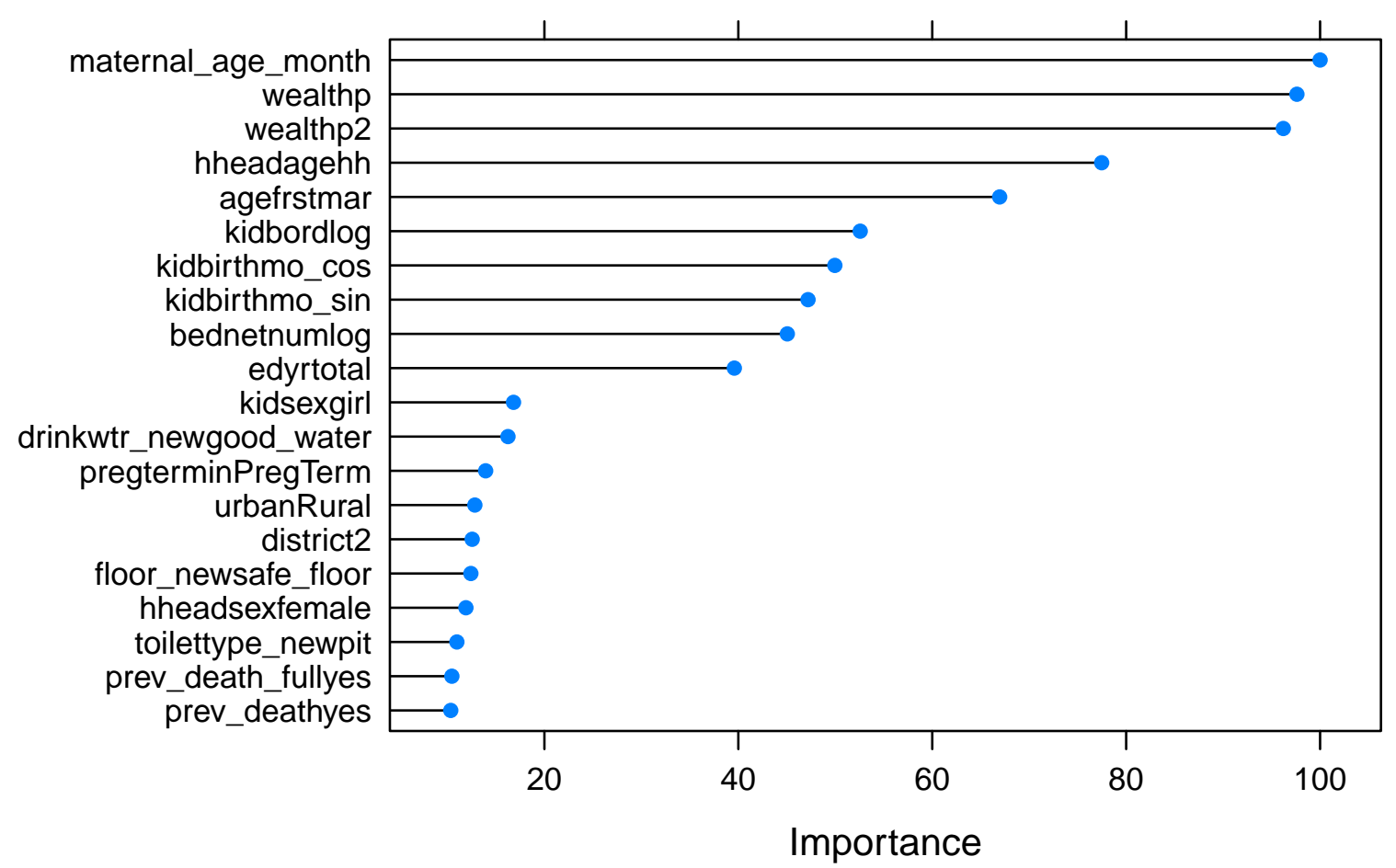

Figure 54: Variable Importance Plot generated by the Random Forest model

\section{B.19.6 Variable Importance Plot}

In this section we will show some measures to give some indication of the important variables necessary for predicting infant mortality. 
medRxiv preprint doi: https://doi.org/10.1101/2021.07.20.21260818; this version posted July 23, 2021. The copyright holder for this preprint (which was not certified by peer review) is the author/funder, who has granted medRxiv a license to display the preprint in perpetuity.

All rights reserved. No reuse allowed without permission.

\section{B.19.7 Elastic Net Coefficients}

Table 152: Standardized coefficients from the optimal elastic net model after 10-fold crossvalidation.

\begin{tabular}{|c|c|c|}
\hline & Coefficients & Standardized Coefficients \\
\hline (Intercept) & -2.8753 & -2.8753 \\
\hline pregterminPregTerm & - & - \\
\hline agefrstmar & - & - \\
\hline hheadagehh & - & - \\
\hline urbanRural & -0.1189 & -0.05 \\
\hline kidsexgirl & -0.2468 & -0.1234 \\
\hline hheadsexfemale & - & - \\
\hline edyrtotal & 0.0081 & 0.0294 \\
\hline drinkwtr_newgood_water & - & - \\
\hline floor_newsafe_floor & - & - \\
\hline maternal_age_month & - & - \\
\hline toilettype_newpit & - & - \\
\hline toilettype_newunimproved & -0.4548 & -0.1633 \\
\hline bednetnumlog & -0.0269 & -0.0173 \\
\hline prev_deathyes & 0.2938 & 0.1087 \\
\hline prev_death_fullyes & - & - \\
\hline kidbordlog & -0.0745 & -0.0533 \\
\hline kidbirthmo_sin & - & - \\
\hline kidbirthmo_cos & -0.1085 & -0.0768 \\
\hline district2 & - & - \\
\hline district3 & -0.0047 & -0.0017 \\
\hline district 4 & -0.0435 & -0.0145 \\
\hline district5 & - & - \\
\hline district 6 & - & - \\
\hline district7 & 0.0894 & 0.0178 \\
\hline district8 & - & - \\
\hline district9 & 0.1317 & 0.0099 \\
\hline district 10 & - & - \\
\hline wealthp & - & - \\
\hline wealthp2 & - & - \\
\hline
\end{tabular}


medRxiv preprint doi: https://doi.org/10.1101/2021.07.20.21260818; this version posted July 23, 2021. The copyright holder for this preprint (which was not certified by peer review) is the author/funder, who has granted medRxiv a license to display the preprint in perpetuity.

All rights reserved. No reuse allowed without permission.

\section{B.20 Uganda}

This sample was taken in the year 2016. There are 11619 observations in the dataset, and 9296 observations were used for the training set. Also, 470 observations were removed due to missing values. Finally, there were 484 deaths for the full dataset, and 388 deaths in the training set. 


\section{B.20.1 Variables Used}

Table 153: Variables included in the model for Uganda

\begin{tabular}{|l|l|}
\hline Variable & Type \\
\hline mortality.under12m & categorical \\
\hline pregtermin & categorical \\
\hline agefrstmar & numeric \\
\hline hheadagehh & numeric \\
\hline urban & categorical \\
\hline kidsex & categorical \\
\hline hheadsex & categorical \\
\hline edyrtotal & numeric \\
\hline drinkwtr_new & categorical \\
\hline floor_new & categorical \\
\hline maternal_age_month & numeric \\
\hline toilettype_new & categorical \\
\hline religion_new & categorical \\
\hline bednetnumlog & numeric \\
\hline prev_death & categorical \\
\hline prev_death_full & categorical \\
\hline kidbordlog & numeric \\
\hline kidbirthmo_sin & numeric \\
\hline kidbirthmo_cos & numeric \\
\hline district & wealthp \\
\hline wealthp2 & numeric \\
\hline categoric
\end{tabular}




\section{B.20.2 Mortality Breakdown by Wealth Quintile}

\section{B.20.3 Optimal Parameters}

These are the algorithm parameters selected after cross-validation:

Table 154: Optimal Parameters for the Elastic Net algorithm

\begin{tabular}{|c|c|}
\hline alpha & lambda \\
\hline 0.8071429 & 0.0062481 \\
\hline
\end{tabular}

Table 155: Optimal Parameters for the Random Forest algorithm

\begin{tabular}{|c|}
\hline mtry \\
\hline 2 \\
\hline
\end{tabular}

Table 156: Optimal Parameters for the XGB algorithm

\begin{tabular}{|c|c|c|c|c|c|c|}
\hline nrounds & max_depth & eta & gamma & colsample_bytree & min_child_weight & subsample \\
\hline 50 & 1 & 0.3 & 0 & 0.6 & 1 & 1 \\
\hline
\end{tabular}

Table 157: Optimal Parameters for the KRLS algorithm

\begin{tabular}{|c|c|c|c|}
\hline loss & epsilon & $\mathbf{b}$ & lambda \\
\hline logistic & 0.005 & 268 & 0.0002704 \\
\hline
\end{tabular}


medRxiv preprint doi: https://doi.org/10.1101/2021.07.20.21260818; this version posted July 23, 2021. The copyright holder for this preprint (which was not certified by peer review) is the author/funder, who has granted medRxiv a license to display the preprint in perpetuity.

\section{B.20.4 Table of Results}

Table 158: Manual Cross-Validation Results

\begin{tabular}{|c|c|c|c|c|c|c|c|c|c|}
\hline Algorithm & Recall 10 & Recall 20 & ROC & AUC & MRD & MRR & MSE & F1 & Efficiency Gain \\
\hline Ensemble & 0.178 & 0.307 & 0.579 & 0.069 & 0.002 & 1.059 & 0.040 & 0.932 & 1.725 \\
\hline KRLS & 0.134 & 0.237 & 0.535 & 0.062 & 0.001 & 1.027 & 0.040 & 0.930 & 1.299 \\
\hline Elastic Net & 0.145 & 0.240 & 0.536 & 0.062 & 0.001 & 1.019 & 0.040 & 0.930 & 1.402 \\
\hline Random Forest & 0.167 & 0.278 & 0.554 & 0.061 & 0.001 & 1.620 & 0.041 & 0.931 & 1.624 \\
\hline Wealth & 0.103 & 0.224 & 0.537 & 0.049 & -0.021 & 0.919 & 0.900 & 0.929 & 1.000 \\
\hline XGB & 0.157 & 0.294 & 0.576 & 0.065 & 0.004 & 1.104 & 0.040 & 0.931 & 1.526 \\
\hline
\end{tabular}

Table 159: Distribution of individuals in the top risk decile of each algorithm among each wealth decile

\begin{tabular}{l|r|r|r|r|c}
\hline Wealth Decile & Elastic Net & Ensemble & KRLS & Random Forest & XGB \\
\hline 1 & 0.229 & 0.165 & 0.235 & 0.150 & 0.125 \\
\hline 2 & 0.136 & 0.094 & 0.075 & 0.091 & 0.099 \\
\hline 3 & 0.117 & 0.081 & 0.082 & 0.107 & 0.086 \\
\hline 4 & 0.112 & 0.092 & 0.076 & 0.081 & 0.097 \\
\hline 5 & 0.082 & 0.087 & 0.081 & 0.091 & 0.095 \\
\hline 6 & 0.097 & 0.083 & 0.104 & 0.082 & 0.071 \\
\hline 7 & 0.086 & 0.090 & 0.105 & 0.093 & 0.086 \\
\hline 8 & 0.068 & 0.129 & 0.103 & 0.116 & 0.143 \\
\hline 9 & 0.046 & 0.103 & 0.087 & 0.109 & 0.102 \\
\hline 10 & 0.026 & 0.079 & 0.051 & 0.080 & 0.095 \\
\hline
\end{tabular}

\section{B.20.5 Performance Plots}

Below are various plots showing the performance for each model considered in Uganda. 
medRxiv preprint doi: https://doi.org/10.1101/2021.07.20.21260818; this version posted July 23, 2021. The copyright holder for this preprint (which was not certified by peer review) is the author/funder, who has granted medRxiv a license to display the preprint in perpetuity. All rights reserved. No reuse allowed without permission.
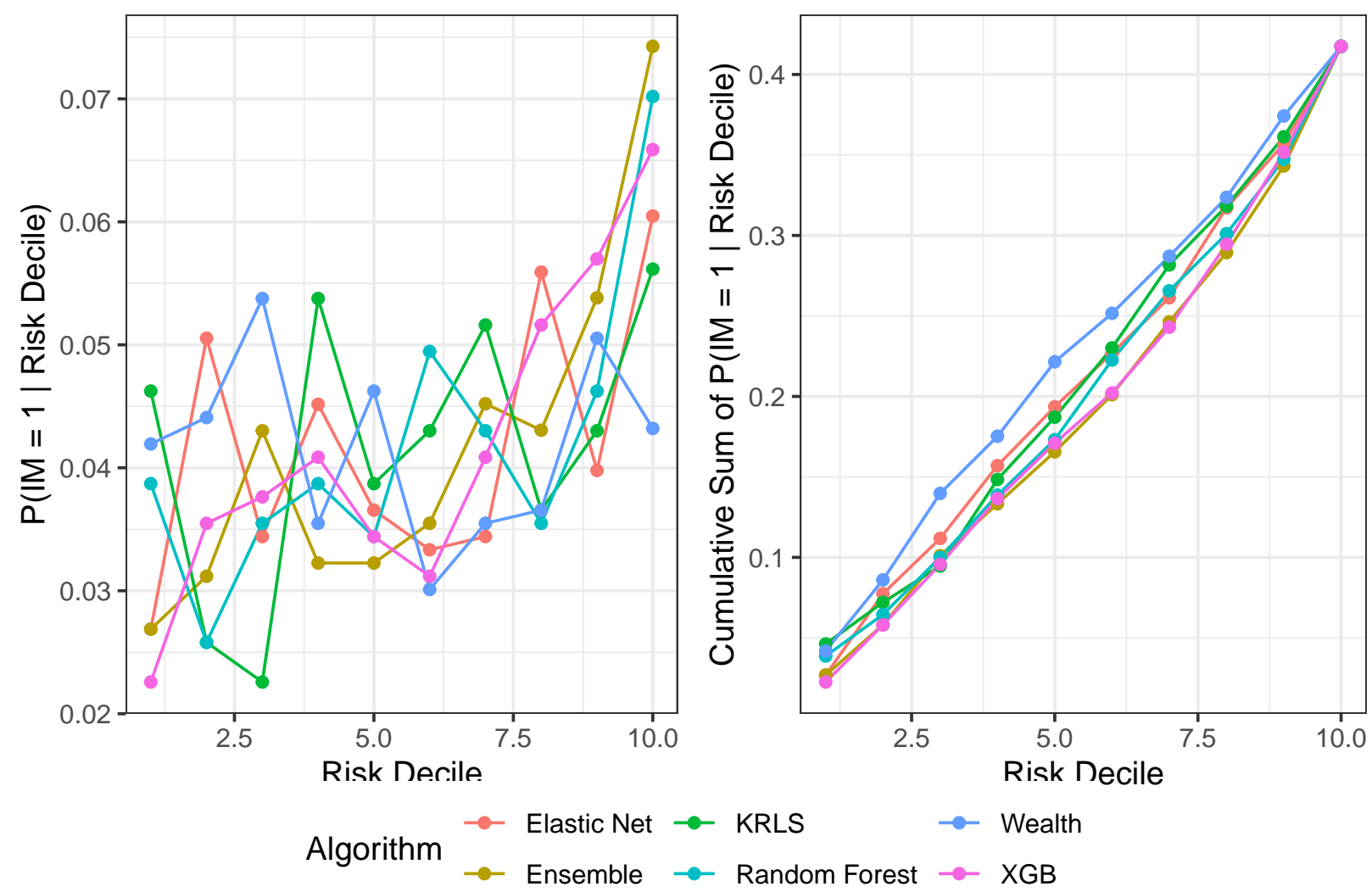

Figure 55: Probability of a mortality given that the observation is in a particular risk decile 
medRxiv preprint doi: https://doi.org/10.1101/2021.07.20.21260818; this version posted July 23, 2021. The copyright holder for this preprint (which was not certified by peer review) is the author/funder, who has granted medRxiv a license to display the preprint in perpetuity. All rights reserved. No reuse allowed without permission.
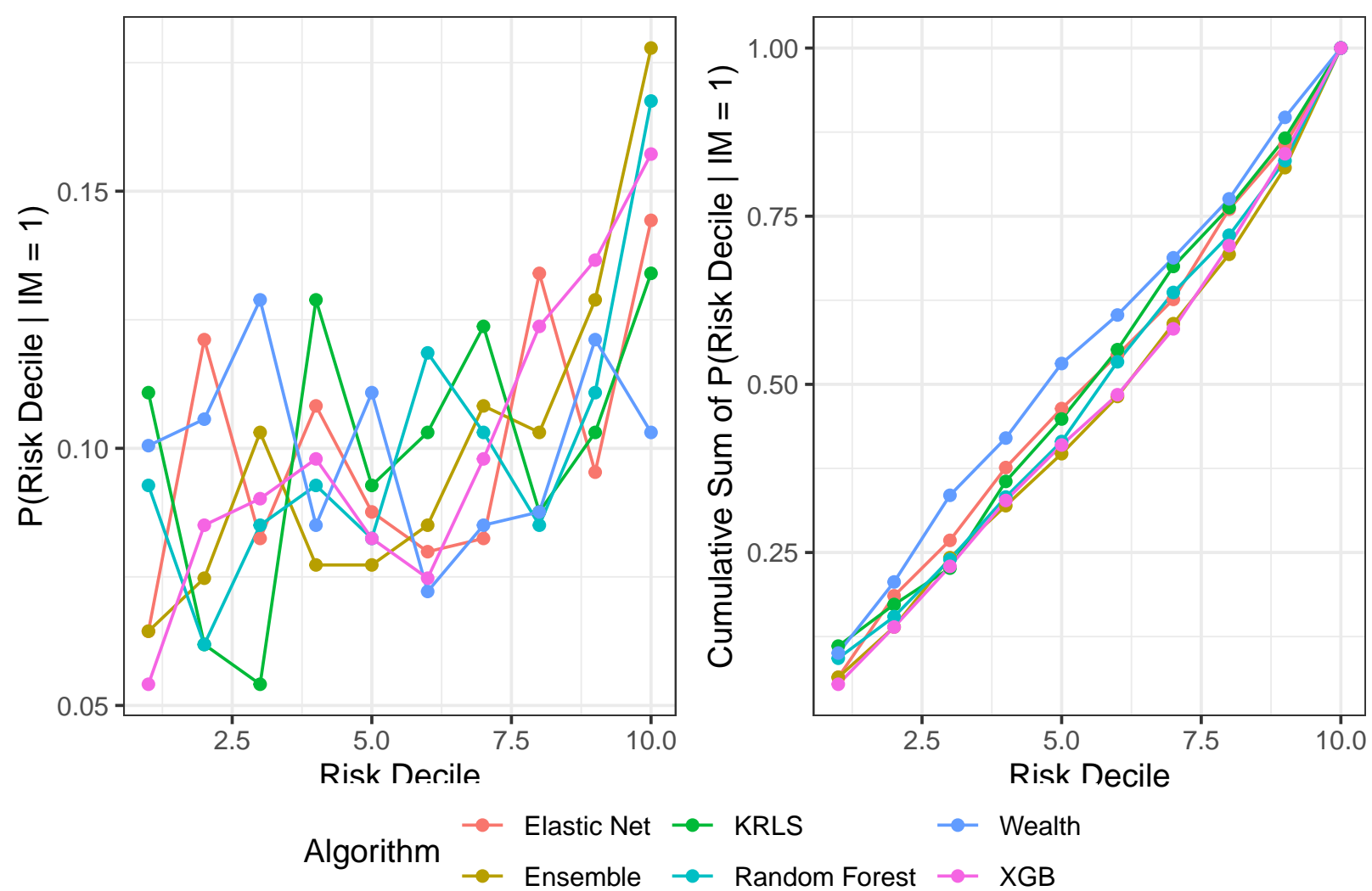

Figure 56: Probability of membership in a particular risk decile given a mortality 


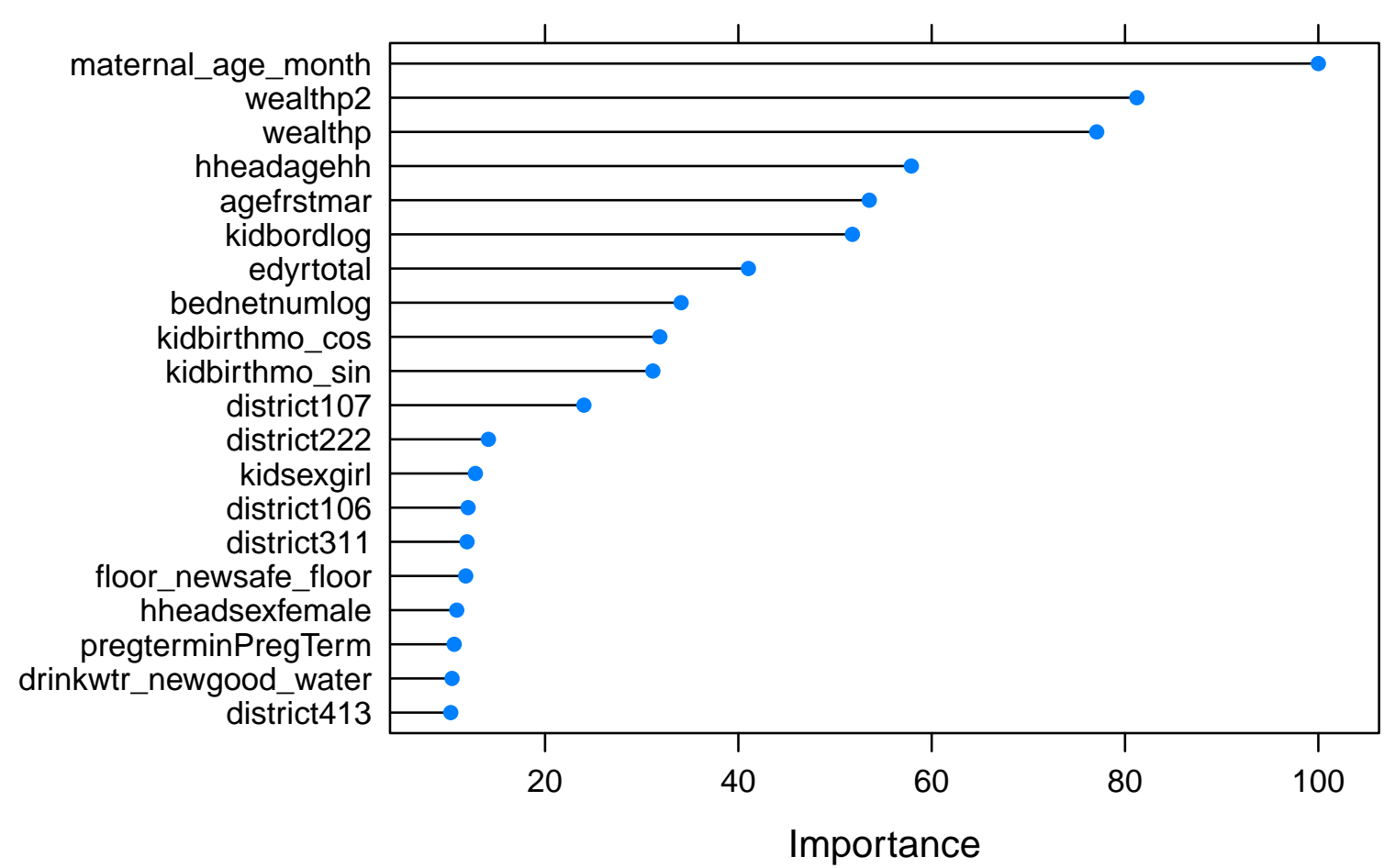

Figure 57: Variable Importance Plot generated by the Random Forest model

\section{B.20.6 Variable Importance Plot}

In this section we will show some measures to give some indication of the important variables necessary for predicting infant mortality. 
medRxiv preprint doi: https://doi.org/10.1101/2021.07.20.21260818; this version posted July 23, 2021. The copyright holder for this preprint (which was not certified by peer review) is the author/funder, who has granted medRxiv a license to display the preprint in perpetuity.

All rights reserved. No reuse allowed without permission.

\section{B.20.7 Elastic Net Coefficients}

Table 160: Standardized coefficients from the optimal elastic net model after 10-fold crossvalidation.

\begin{tabular}{|c|c|c|}
\hline & Coefficients & Standardized Coefficients \\
\hline (Intercept) & -3.0915 & -3.0915 \\
\hline pregterminPregTerm & - & - \\
\hline agefrstmar & - & - \\
\hline hheadagehh & - & - \\
\hline urbanRural & - & - \\
\hline kidsexgirl & -0.0042 & -0.0021 \\
\hline hheadsexfemale & - & - \\
\hline edyrtotal & -0.0086 & -0.0331 \\
\hline drinkwtr_newgood_water & - & - \\
\hline floor_newsafe_floor & - & - \\
\hline maternal_age_month & - & - \\
\hline toilettype_newpit & - & - \\
\hline toilettype_newunimproved & - & - \\
\hline religion_newChristian & - & - \\
\hline religion_newHindu & - & - \\
\hline religion_newMuslim & - & - \\
\hline religion_newOther & - & - \\
\hline bednetnumlog & - & - \\
\hline prev_deathyes & - & - \\
\hline prev_death_fullyes & - & - \\
\hline kidbordlog & - & - \\
\hline kidbirthmo_sin & - & - \\
\hline kidbirthmo_cos & - & - \\
\hline district102 & - & - \\
\hline district 103 & - & - \\
\hline district 104 & - & - \\
\hline district 105 & - & - \\
\hline district 106 & - & - \\
\hline district 107 & 0.7873 & 0.0828 \\
\hline district108 & - & - \\
\hline district 109 & - & - \\
\hline district 110 & - & - \\
\hline district 111 & - & - \\
\hline district 112 & - & - \\
\hline district 113 & - & - \\
\hline district 114 & - & - \\
\hline district 115 & - & - \\
\hline district 116 & - & - \\
\hline district 117 & - & - \\
\hline
\end{tabular}


medRxiv preprint doi: https://doi.org/10.1101/2021.07.20.21260818; this version posted July 23, 2021. The copyright holder for this preprint (which was not certified by peer review) is the author/funder, who has granted medRxiv a license to display the preprint in perpetuity.

All rights reserved. No reuse allowed without permission.

Table 160: Standardized coefficients from the optimal elastic net model after 10-fold crossvalidation. (continued)

\begin{tabular}{|c|c|c|}
\hline & Coefficients & Standardized Coefficients \\
\hline district118 & - & - \\
\hline district119 & - & - \\
\hline district 120 & - & - \\
\hline district121 & - & - \\
\hline $\operatorname{district} 122$ & - & - \\
\hline district123 & - & - \\
\hline district 124 & - & - \\
\hline district 201 & - & - \\
\hline district202 & - & - \\
\hline district203 & - & - \\
\hline district 204 & - & - \\
\hline district 205 & - & - \\
\hline district 206 & - & - \\
\hline district 207 & - & - \\
\hline district 208 & - & - \\
\hline district209 & - & - \\
\hline district 210 & - & - \\
\hline district 211 & - & - \\
\hline district 212 & - & - \\
\hline district 213 & - & - \\
\hline district 214 & - & - \\
\hline district 215 & - & - \\
\hline district 216 & - & - \\
\hline district 217 & - & - \\
\hline district 218 & - & - \\
\hline district 219 & - & - \\
\hline district 220 & - & - \\
\hline district 221 & - & - \\
\hline district 222 & 0.4808 & 0.0362 \\
\hline district 223 & - & - \\
\hline district 224 & - & - \\
\hline district 225 & - & - \\
\hline district 226 & - & - \\
\hline district 227 & - & - \\
\hline district 228 & - & - \\
\hline district229 & - & - \\
\hline district 230 & - & - \\
\hline district 231 & - & - \\
\hline district 232 & - & - \\
\hline district301 & - & - \\
\hline district302 & - & - \\
\hline
\end{tabular}


medRxiv preprint doi: https://doi.org/10.1101/2021.07.20.21260818; this version posted July 23, 2021. The copyright holder for this preprint (which was not certified by peer review) is the author/funder, who has granted medRxiv a license to display the preprint in perpetuity.

All rights reserved. No reuse allowed without permission.

Table 160: Standardized coefficients from the optimal elastic net model after 10-fold crossvalidation. (continued)

\begin{tabular}{|c|c|c|}
\hline & Coefficients & Standardized Coefficients \\
\hline district303 & - & - \\
\hline district304 & - & - \\
\hline district305 & - & - \\
\hline district306 & - & - \\
\hline district307 & - & - \\
\hline district308 & - & - \\
\hline district309 & - & - \\
\hline district310 & - & - \\
\hline district311 & - & - \\
\hline district312 & - & - \\
\hline district313 & - & - \\
\hline district314 & - & - \\
\hline district315 & - & - \\
\hline district316 & - & - \\
\hline district317 & - & - \\
\hline district318 & - & - \\
\hline district319 & - & - \\
\hline district 320 & - & - \\
\hline district321 & - & - \\
\hline district322 & - & - \\
\hline district323 & - & - \\
\hline district324 & - & - \\
\hline district325 & - & - \\
\hline district326 & - & - \\
\hline district327 & - & - \\
\hline district328 & - & - \\
\hline district329 & - & - \\
\hline district330 & - & - \\
\hline district 401 & - & - \\
\hline district 402 & - & - \\
\hline district 403 & - & - \\
\hline district 404 & - & - \\
\hline district 405 & - & - \\
\hline district406 & - & - \\
\hline district 407 & - & - \\
\hline district408 & - & - \\
\hline district409 & - & - \\
\hline district 410 & - & - \\
\hline district 411 & - & - \\
\hline district 412 & - & - \\
\hline district 413 & 0.0056 & $5 \mathrm{e}-04$ \\
\hline
\end{tabular}


medRxiv preprint doi: https://doi.org/10.1101/2021.07.20.21260818; this version posted July 23, 2021. The copyright holder for this preprint (which was not certified by peer review) is the author/funder, who has granted medRxiv a license to display the preprint in perpetuity.

All rights reserved. No reuse allowed without permission.

Table 160: Standardized coefficients from the optimal elastic net model after 10-fold crossvalidation. (continued)

\begin{tabular}{|l|c|c|}
\hline & Coefficients & Standardized Coefficients \\
\hline district414 & - & - \\
\hline district415 & - & - \\
\hline district416 & - & - \\
\hline district417 & - & - \\
\hline district418 & - & - \\
\hline district419 & - & - \\
\hline district420 & - & - \\
\hline district421 & - & - \\
\hline district422 & - & - \\
\hline district423 & - & - \\
\hline district424 & - & - \\
\hline district425 & - & - \\
\hline district426 & - & - \\
\hline wealthp & - & - \\
\hline wealthp2 & - & - \\
\hline
\end{tabular}


medRxiv preprint doi: https://doi.org/10.1101/2021.07.20.21260818; this version posted July 23, 2021. The copyright holder for this preprint (which was not certified by peer review) is the author/funder, who has granted medRxiv a license to display the preprint in perpetuity.

All rights reserved. No reuse allowed without permission.

\section{B.21 Zambia}

This sample was taken in the year 2013. There are 9811 observations in the dataset, and 7850 observations were used for the training set. Also, 835 observations were removed due to missing values. Finally, there were 483 deaths for the full dataset, and 387 deaths in the training set. 
medRxiv preprint doi: https://doi.org/10.1101/2021.07.20.21260818; this version posted July 23, 2021. The copyright holder for this preprint (which was not certified by peer review) is the author/funder, who has granted medRxiv a license to display the preprint in perpetuity.

All rights reserved. No reuse allowed without permission. 


\section{B.21.1 Variables Used}

Table 161: Variables included in the model for Zambia

\begin{tabular}{|c|c|}
\hline Variable & Type \\
\hline mortality.under $12 \mathrm{~m}$ & categorical \\
\hline pregtermin & categorical \\
\hline agefrstmar & numeric \\
\hline hheadagehh & numeric \\
\hline urban & categorical \\
\hline kidsex & categorical \\
\hline hheadsex & categorical \\
\hline edyrtotal & numeric \\
\hline drinkwtr_new & categorical \\
\hline floor_new & categorical \\
\hline maternal_age_month & numeric \\
\hline cookfuel_new & categorical \\
\hline toilettype_new & categorical \\
\hline religion_new & categorical \\
\hline bednetnumlog & numeric \\
\hline prev_death & categorical \\
\hline prev_death_full & categorical \\
\hline malaria_new & numeric \\
\hline kidbordlog & numeric \\
\hline kidbirthmo_sin & numeric \\
\hline kidbirthmo_cos & numeric \\
\hline wealthp & numeric \\
\hline wealthp2 & numeric \\
\hline
\end{tabular}




\section{B.21.2 Mortality Breakdown by Wealth Quintile}

\section{B.21.3 Optimal Parameters}

These are the algorithm parameters selected after cross-validation:

Table 162: Optimal Parameters for the Elastic Net algorithm

\begin{tabular}{|c|c|}
\hline alpha & lambda \\
\hline 0.1 & 0.004132 \\
\hline
\end{tabular}

Table 163: Optimal Parameters for the Random Forest algorithm

\begin{tabular}{|c|}
\hline mtry \\
\hline 2 \\
\hline
\end{tabular}

Table 164: Optimal Parameters for the XGB algorithm

\begin{tabular}{|c|c|c|c|c|c|c|}
\hline nrounds & max_depth & eta & gamma & colsample_bytree & min_child_weight & subsample \\
\hline 50 & 1 & 0.3 & 0 & 0.6 & 1 & 1 \\
\hline
\end{tabular}

Table 165: Optimal Parameters for the KRLS algorithm

\begin{tabular}{|c|c|c|c|}
\hline loss & epsilon & $\mathbf{b}$ & lambda \\
\hline logistic & 0.005 & 50 & 0.0001824 \\
\hline
\end{tabular}


medRxiv preprint doi: https://doi.org/10.1101/2021.07.20.21260818; this version posted July 23, 2021. The copyright holder for this preprint (which was not certified by peer review) is the author/funder, who has granted medRxiv a license to display the preprint in perpetuity.

\section{B.21.4 Table of Results}

Table 166: Manual Cross-Validation Results

\begin{tabular}{|c|c|c|c|c|c|c|c|c|c|}
\hline Algorithm & Recall 10 & Recall 20 & ROC & AUC & MRD & MRR & MSE & F1 & Efficiency Gain \\
\hline Ensemble & 0.209 & 0.354 & 0.617 & 0.094 & 0.008 & 1.190 & 0.046 & 0.930 & 2.619 \\
\hline KRLS & 0.186 & 0.333 & 0.588 & 0.083 & 0.005 & 1.098 & 0.047 & 0.930 & 2.327 \\
\hline Elastic Net & 0.150 & 0.310 & 0.579 & 0.070 & 0.005 & 1.099 & 0.047 & 0.928 & 1.875 \\
\hline Random Forest & 0.209 & 0.300 & 0.598 & 0.101 & 0.016 & 1.588 & 0.047 & 0.931 & 2.618 \\
\hline Wealth & 0.080 & 0.163 & 0.529 & 0.051 & -0.009 & 0.990 & 0.817 & 0.924 & 1.000 \\
\hline XGB & 0.194 & 0.320 & 0.595 & 0.080 & 0.008 & 1.160 & 0.047 & 0.930 & 2.422 \\
\hline
\end{tabular}

Table 167: Distribution of individuals in the top risk decile of each algorithm among each wealth decile

\begin{tabular}{l|r|r|r|r|r}
\hline Wealth Decile & Elastic Net & Ensemble & KRLS & Random Forest & XGB \\
\hline 1 & 0.071 & 0.117 & 0.095 & 0.149 & 0.100 \\
\hline 2 & 0.079 & 0.092 & 0.103 & 0.082 & 0.090 \\
\hline 3 & 0.072 & 0.087 & 0.083 & 0.074 & 0.113 \\
\hline 4 & 0.078 & 0.078 & 0.087 & 0.082 & 0.088 \\
\hline 5 & 0.063 & 0.094 & 0.072 & 0.106 & 0.112 \\
\hline 6 & 0.104 & 0.118 & 0.095 & 0.108 & 0.128 \\
\hline 7 & 0.130 & 0.101 & 0.106 & 0.074 & 0.102 \\
\hline 8 & 0.142 & 0.105 & 0.123 & 0.073 & 0.112 \\
\hline 9 & 0.162 & 0.128 & 0.141 & 0.128 & 0.101 \\
\hline 10 & 0.099 & 0.086 & 0.094 & 0.123 & 0.053 \\
\hline
\end{tabular}

\section{B.21.5 Performance Plots}

Below are various plots showing the performance for each model considered in Zambia. 
medRxiv preprint doi: https://doi.org/10.1101/2021.07.20.21260818; this version posted July 23, 2021. The copyright holder for this preprint (which was not certified by peer review) is the author/funder, who has granted medRxiv a license to display the preprint in perpetuity. All rights reserved. No reuse allowed without permission.

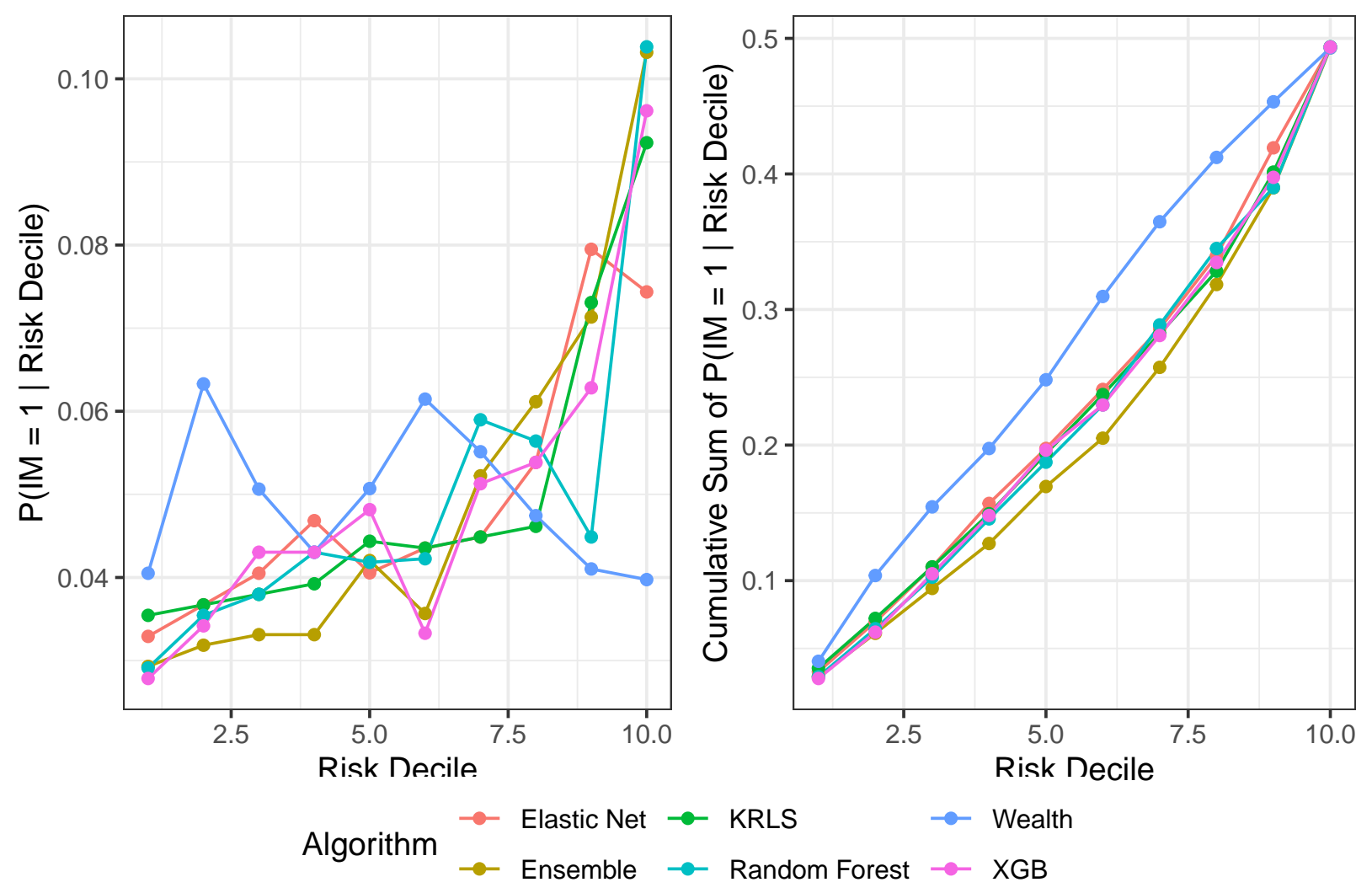

Figure 58: Probability of a mortality given that the observation is in a particular risk decile 
medRxiv preprint doi: https://doi.org/10.1101/2021.07.20.21260818; this version posted July 23, 2021. The copyright holder for this preprint (which was not certified by peer review) is the author/funder, who has granted medRxiv a license to display the preprint in perpetuity. All rights reserved. No reuse allowed without permission.

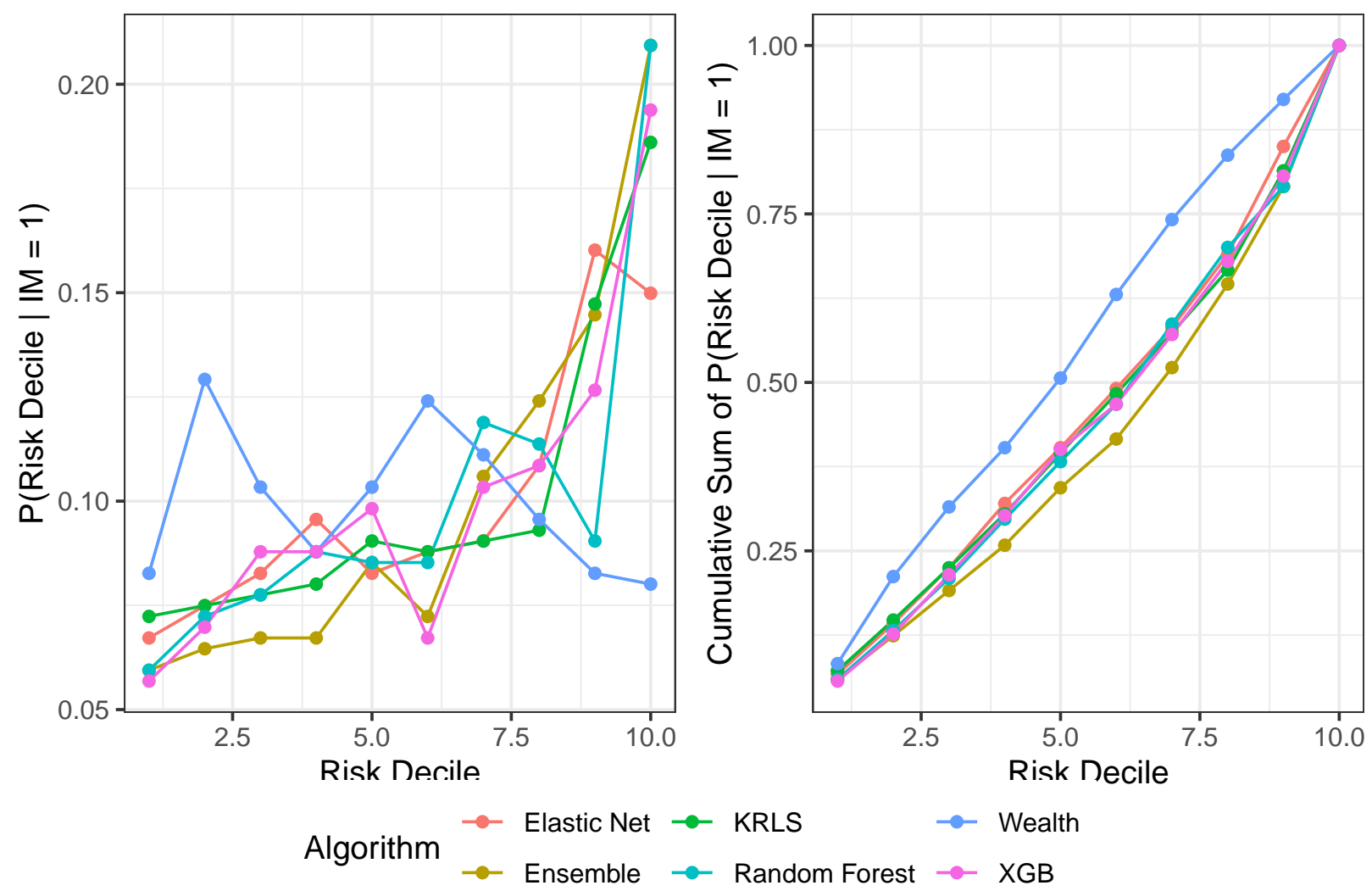

Figure 59: Probability of membership in a particular risk decile given a mortality 


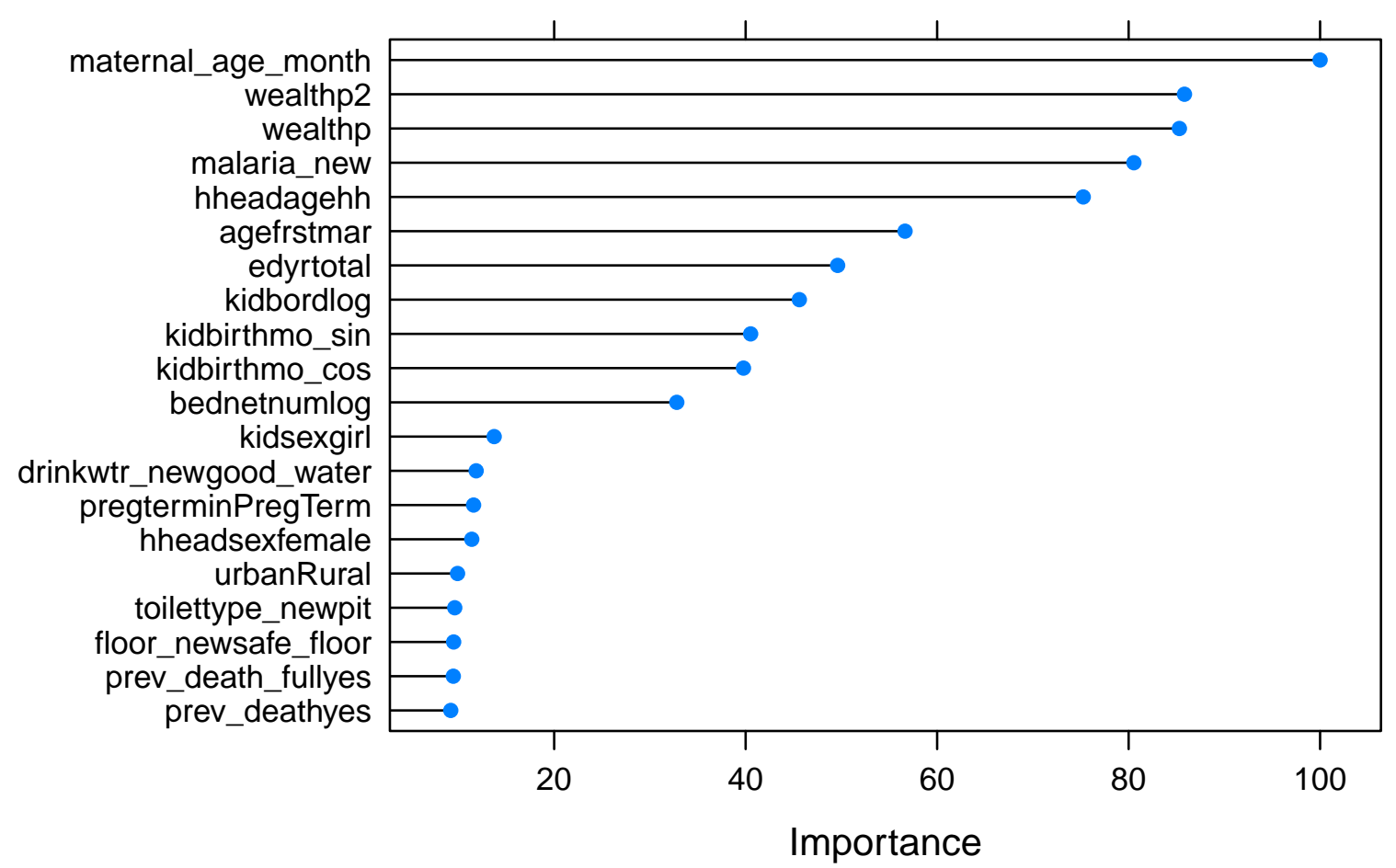

Figure 60: Variable Importance Plot generated by the Random Forest model

\section{B.21.6 Variable Importance Plot}

In this section we will show some measures to give some indication of the important variables necessary for predicting infant mortality. 
medRxiv preprint doi: https://doi.org/10.1101/2021.07.20.21260818; this version posted July 23, 2021. The copyright holder for this preprint (which was not certified by peer review) is the author/funder, who has granted medRxiv a license to display the preprint in perpetuity.

All rights reserved. No reuse allowed without permission.

\section{B.21.7 Elastic Net Coefficients}

Table 168: Standardized coefficients from the optimal elastic net model after 10-fold crossvalidation.

\begin{tabular}{|l|c|c|}
\hline & Coefficients & Standardized Coefficients \\
\hline (Intercept) & -1.6512 & -1.6512 \\
\hline pregterminPregTerm & 0.254 & 0.0817 \\
\hline agefrstmar & -0.0315 & -0.1057 \\
\hline hheadagehh & 0.0101 & 0.1065 \\
\hline urbanRural & -0.1266 & -0.0607 \\
\hline kidsexgirl & -0.2211 & -0.1106 \\
\hline hheadsexfemale & 0.1064 & 0.0401 \\
\hline edyrtotal & -0.0231 & -0.0828 \\
\hline drinkwtr_newgood_water & 0.0777 & 0.0385 \\
\hline floor_newsafe_floor & 0.0405 & 0.0188 \\
\hline maternal_age_month & - & - \\
\hline cookfuel_newclean_fuel & -0.2157 & -0.0536 \\
\hline toilettype_newpit & - & - \\
\hline toilettype_newunimproved & - & - \\
\hline religion_newChristian & -0.5262 & -0.061 \\
\hline religion_newHindu & - & - \\
\hline religion_newMuslim & 0.4863 & 0.0295 \\
\hline religion_newOther & 0.0276 & 0.0027 \\
\hline bednetnumlog & -0.1409 & -0.0733 \\
\hline prev_deathyes & 0.2981 & 0.1149 \\
\hline prev_death_fullyes & 0.1422 & 0.0619 \\
\hline malaria_new & 0.055 & 0.0071 \\
\hline kidbordlog & -0.3687 & -0.252 \\
\hline kidbirthmo_sin & -0.1058 & - \\
\hline kidbirthmo_cos & 0.0662 & 0.0396 \\
\hline wealthp & 0.1363 & - \\
\hline wealthp2 & & \\
\hline
\end{tabular}


medRxiv preprint doi: https://doi.org/10.1101/2021.07.20.21260818; this version posted July 23, 2021. The copyright holder for this preprint (which was not certified by peer review) is the author/funder, who has granted medRxiv a license to display the preprint in perpetuity.

All rights reserved. No reuse allowed without permission.

\section{B.22 Zimbabwe}

This sample was taken in the year 2015. There are 4630 observations in the dataset, and 3705 observations were used for the training set. Also, 222 observations were removed due to missing values. Finally, there were 236 deaths for the full dataset, and 189 deaths in the training set. 
medRxiv preprint doi: https://doi.org/10.1101/2021.07.20.21260818; this version posted July 23, 2021. The copyright holder for this preprint (which was not certified by peer review) is the author/funder, who has granted medRxiv a license to display the preprint in perpetuity.

All rights reserved. No reuse allowed without permission. 


\section{B.22.1 Variables Used}

Table 169: Variables included in the model for Zimbabwe

\begin{tabular}{|l|l|}
\hline Variable & Type \\
\hline mortality.under12m & categorical \\
\hline pregtermin & categorical \\
\hline agefrstmar & numeric \\
\hline hheadagehh & numeric \\
\hline urban & categorical \\
\hline kidsex & categorical \\
\hline hheadsex & categorical \\
\hline edyrtotal & numeric \\
\hline drinkwtr_new & categorical \\
\hline floor_new & categorical \\
\hline maternal_age_month & numeric \\
\hline cookfuel_new & categorical \\
\hline toilettype_new & categorical \\
\hline religion_new & categorical \\
\hline bednetnumlog & numeric \\
\hline prev_death & categorical \\
\hline prev_death_full & categorical \\
\hline kidbordlog & numeric \\
\hline kidbirthmo_sin & numeric \\
\hline kidbirthmo_cos & numeric \\
\hline geo_zw2015 & categorical \\
\hline wealthp & numealthp2 \\
\hline wic
\end{tabular}




\section{B.22.2 Mortality Breakdown by Wealth Quintile}

\section{B.22.3 Optimal Parameters}

These are the algorithm parameters selected after cross-validation:

Table 170: Optimal Parameters for the Elastic Net algorithm

\begin{tabular}{|c|c|}
\hline alpha & lambda \\
\hline 1 & 0.0030947 \\
\hline
\end{tabular}

Table 171: Optimal Parameters for the Random Forest algorithm

\begin{tabular}{|c|}
\hline mtry \\
\hline 2 \\
\hline
\end{tabular}

Table 172: Optimal Parameters for the XGB algorithm

\begin{tabular}{|c|c|c|c|c|c|c|}
\hline nrounds & max_depth & eta & gamma & colsample_bytree & min_child_weight & subsample \\
\hline 100 & 1 & 0.3 & 0 & 0.8 & 1 & 1 \\
\hline
\end{tabular}

Table 173: Optimal Parameters for the KRLS algorithm

\begin{tabular}{|c|c|c|c|}
\hline loss & epsilon & $\mathbf{b}$ & lambda \\
\hline logistic & 0.005 & 66 & 0.000283 \\
\hline
\end{tabular}


medRxiv preprint doi: https://doi.org/10.1101/2021.07.20.21260818; this version posted July 23, 2021. The copyright holder for this preprint (which was not certified by peer review) is the author/funder, who has granted medRxiv a license to display the preprint in perpetuity.

\section{B.22.4 Table of Results}

Table 174: Manual Cross-Validation Results

\begin{tabular}{|c|c|c|c|c|c|c|c|c|c|}
\hline Algorithm & Recall 10 & Recall 20 & ROC & AUC & MRD & MRR & MSE & F1 & Efficiency Gain \\
\hline Ensemble & 0.190 & 0.333 & 0.642 & 0.104 & 0.010 & 1.216 & 0.048 & 0.929 & 1.569 \\
\hline KRLS & 0.154 & 0.292 & 0.600 & 0.076 & 0.005 & 1.108 & 0.048 & 0.927 & 1.265 \\
\hline Elastic Net & 0.143 & 0.308 & 0.576 & 0.077 & 0.005 & 1.095 & 0.048 & 0.926 & 1.176 \\
\hline Random Forest & 0.201 & 0.338 & 0.629 & 0.116 & 0.019 & 1.726 & 0.048 & 0.930 & 1.653 \\
\hline Wealth & 0.121 & 0.228 & 0.570 & 0.065 & 2.029 & -170.854 & 89.014 & 0.925 & 1.000 \\
\hline XGB & 0.175 & 0.318 & 0.588 & 0.081 & 0.009 & 1.184 & 0.048 & 0.928 & 1.441 \\
\hline
\end{tabular}

Table 175: Distribution of individuals in the top risk decile of each algorithm among each wealth decile

\begin{tabular}{l|r|r|r|r|r}
\hline Wealth Decile & Elastic Net & Ensemble & KRLS & Random Forest & XGB \\
\hline 1 & 0.274 & 0.214 & 0.222 & 0.201 & 0.160 \\
\hline 2 & 0.197 & 0.181 & 0.197 & 0.154 & 0.170 \\
\hline 3 & 0.187 & 0.168 & 0.206 & 0.108 & 0.173 \\
\hline 4 & 0.149 & 0.152 & 0.179 & 0.100 & 0.141 \\
\hline 5 & 0.068 & 0.103 & 0.092 & 0.092 & 0.092 \\
\hline 6 & 0.065 & 0.092 & 0.073 & 0.114 & 0.106 \\
\hline 7 & 0.022 & 0.035 & 0.016 & 0.073 & 0.049 \\
\hline 8 & 0.016 & 0.011 & 0.008 & 0.054 & 0.014 \\
\hline 9 & 0.016 & 0.038 & 0.005 & 0.068 & 0.076 \\
\hline 10 & 0.005 & 0.008 & 0.000 & 0.035 & 0.019 \\
\hline
\end{tabular}

\section{B.22.5 Performance Plots}

Below are various plots showing the performance for each model considered in Zimbabwe. 
medRxiv preprint doi: https://doi.org/10.1101/2021.07.20.21260818; this version posted July 23, 2021. The copyright holder for this preprint (which was not certified by peer review) is the author/funder, who has granted medRxiv a license to display the preprint in perpetuity. All rights reserved. No reuse allowed without permission.

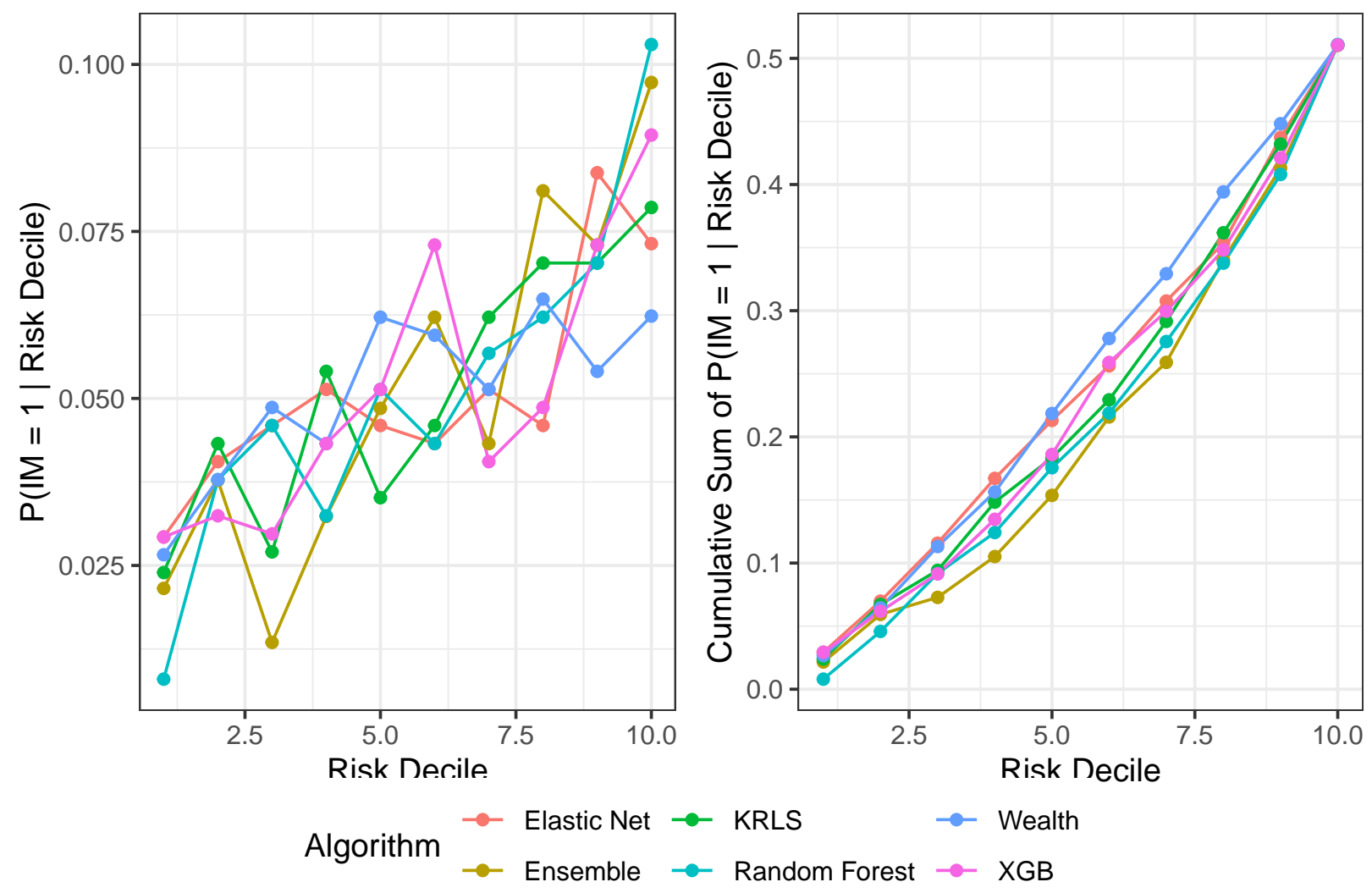

Figure 61: Probability of a mortality given that the observation is in a particular risk decile 
medRxiv preprint doi: https://doi.org/10.1101/2021.07.20.21260818; this version posted July 23, 2021. The copyright holder for this preprint (which was not certified by peer review) is the author/funder, who has granted medRxiv a license to display the preprint in perpetuity. All rights reserved. No reuse allowed without permission.
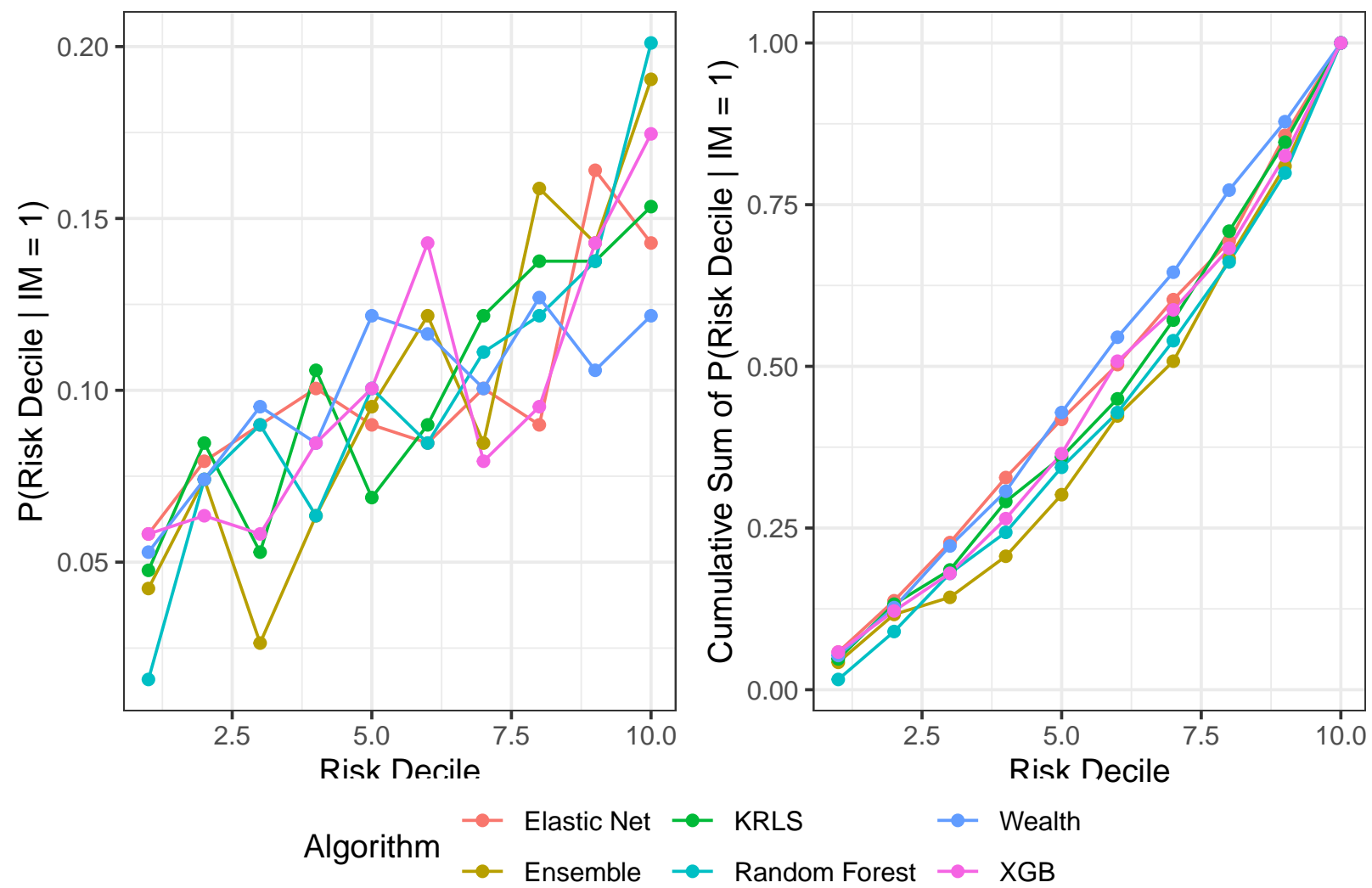

Figure 62: Probability of membership in a particular risk decile given a mortality 


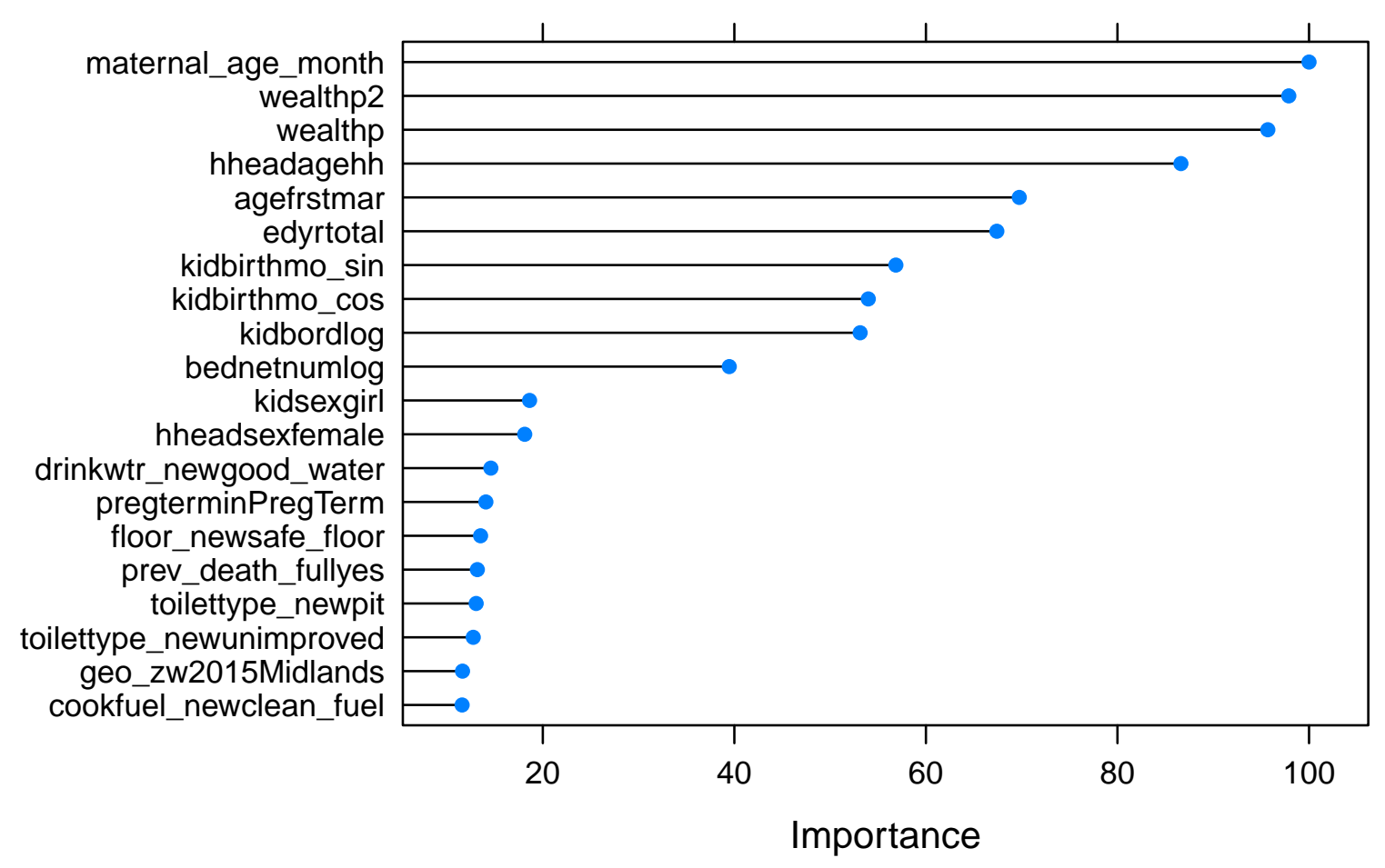

Figure 63: Variable Importance Plot generated by the Random Forest model

\section{B.22.6 Variable Importance Plot}

In this section we will show some measures to give some indication of the important variables necessary for predicting infant mortality. 
medRxiv preprint doi: https://doi.org/10.1101/2021.07.20.21260818; this version posted July 23, 2021. The copyright holder for this preprint (which was not certified by peer review) is the author/funder, who has granted medRxiv a license to display the preprint in perpetuity.

All rights reserved. No reuse allowed without permission.

\section{B.22.7 Elastic Net Coefficients}

Table 176: Standardized coefficients from the optimal elastic net model after 10-fold crossvalidation.

\begin{tabular}{|c|c|c|}
\hline & Coefficients & Standardized Coefficients \\
\hline (Intercept) & -2.1829 & -2.1829 \\
\hline pregterminPregTerm & 0.0089 & 0.003 \\
\hline agefrstmar & -0.0021 & -0.0076 \\
\hline hheadagehh & - & - \\
\hline urbanRural & 0.0719 & 0.0349 \\
\hline kidsexgirl & -0.1007 & -0.0504 \\
\hline hheadsexfemale & 0.1601 & 0.0768 \\
\hline edyrtotal & -0.0861 & -0.2474 \\
\hline drinkwtr_newgood_water & 0.0814 & 0.0349 \\
\hline floor_newsafe_floor & - & - \\
\hline maternal_age_month & - & - \\
\hline cookfuel_newclean_fuel & - & - \\
\hline toilettype_newpit & - & - \\
\hline toilettype_newunimproved & 0.2206 & 0.097 \\
\hline religion_newChristian & 0.151 & 0.0384 \\
\hline religion_newHindu & - & - \\
\hline religion_newMuslim & - & - \\
\hline religion_newOther & - & - \\
\hline bednetnumlog & - & - \\
\hline prev_deathyes & 0.0259 & 0.0084 \\
\hline prev_death_fullyes & 0.2783 & 0.0994 \\
\hline kidbordlog & -0.2526 & -0.1527 \\
\hline kidbirthmo_sin & 0.0561 & 0.0396 \\
\hline kidbirthmo_cos & - & - \\
\hline geo_zw2015Mashonaland Central & -0.1399 & -0.0452 \\
\hline geo_zw2015Mashonaland East & - & - \\
\hline geo_zw2015Mashonaland West & - & - \\
\hline geo_zw2015Matabeleland North & -0.02 & -0.0053 \\
\hline geo_zw2015Matabeleland South & - & - \\
\hline geo_zw2015Midlands & - & - \\
\hline geo_zw2015Masvingo & - & - \\
\hline geo_zw2015Harare & - & - \\
\hline geo_zw2015Bulawayo & - & - \\
\hline wealthp & - & - \\
\hline wealthp2 & - & - \\
\hline
\end{tabular}

